\title{
Spectral Theory of Automorphism Groups and Particle Structures in Quantum Field Theory
}

\author{
Dissertation \\ zur Erlangung des Doktorgrades \\ der Mathematisch-Naturwissenschaftlichen Fakultäten \\ der Georg-August-Universität zu Göttingen
}

vorgelegt von

Wojciech Jan Dybalski

aus Warszawa

Göttingen, 2008 
D 7

Referent: Prof. Dr. D. Buchholz

Korreferent: Prof. Dr. K. Fredenhagen

Tag der mündlichen Prüfung: 15.12.2008 


\section{Contents}

1 Introduction $\quad 5$

1.1 Particle Content in Quantum Mechanics. Spectrum of Hamiltonian . . . . . 6

1.2 Wigner's Particle Concept and its Limitations . . . . . . . . . . . . . . . . 6

1.3 Bevond Wigner's Particle Concept. Arveson Spectrum . . . . . . . . . . . . 7

1.4 Detailed Theorv of Arveson Spectrum in Literature . . . . . . . . . . . . . . 10

1.5 Overview of this Work . . . . . . . . . . . . . . . . . . 12

1.6 Technical Backoround . . . . . . . . . . . . . . . . . . 13

\begin{tabular}{|lll}
2 & Spectral Decomposition and Existence of Particles & 17
\end{tabular}

2.1 Space Translations in Quantum Mechanics . . . . . . . . . . . . . . . . 18

2.2 Space Translations in Quantum Field Theorv. Spectral Decomposition . . . 20

2.3 Triviality of Point-Continuous Subspace and Existence of Particles . . . . . 26

3 Uniqueness of Spectral Decomposition and Vacuum Structure 33

3.1 Condition $C_{\sharp}$ and Existence of Vacuum States . . . . . . . . . . . . . 34

3.2 Condition $C_{\sharp}$ : Coincidence Measurement Formulation . . . . . . . . . . 38

3.3 Condition $C_{b}$ and Uniqueness of Vacuum . . . . . . . . . . . 42

3.4 Condition $C_{\mathrm{h}}$ : Additivity of Energy $\ldots \ldots \ldots \ldots$. . . . . . . . . . . 48

3.5 Condition $N_{t}$ implies Condition $C_{t} \ldots \ldots \ldots \ldots \ldots$. . . . . . . . 53

4 Conclusions and Outlook $\quad 59$

A Haag-Ruelle Scattering Theorv in Presence of Massless Particles $\quad 63$

A.1 Introduction . . . . . . . . . . . . . . . . . . . 63

A.2 Existence of Asvmptotic States . . . . . . . . . . . . . . 66

A.3 Fock Structure of Asvmptotic States . . . . . . . . . . . . . . . . . . 70

A.4 Conclusion . . . . . . . . . . . . . . . . . . . 73

B Scalar Free Field Theory and its Phase Space Structure $\quad 75$

B.1 Multiindex Notation . . . . . . . . . . . . . . . . . . 76

B.2 $\quad$ Scalar Free Field Theorv and Related Models . . . . . . . . . . . . . . . . 76

B.3 Special Functionals on Local Algebra . . . . . . . . . . . . . . . . . . . . . 81

B.4 Expansions in Single-Particle Space . . . . . . . . . . . . . . . . . . 83

B.5 Expansion of $\Theta_{E}$ into Rank-One Mappings . . . . . . . . . . . . . . . 88 
C Verification of Condition $L^{(2)}$ in Scalar Free Field Theorv $\quad 95$

C.1 Proofs of Theorems 2.2.5. 2.2.6 and 2.2.7 based on Theorem C.1.1 . . . . 95

C.2 Proof of Theorem C.1.1 (I): Functionals $\left\{\tau_{i}\right\}^{3} \ldots \ldots \ldots 1$

C.3 Proof of Theorem C.1.1 (II): Square-Integrability of $R^{(2)} \ldots$. . . . . . . 104

D Verification of Condition $L_{\sharp}^{(1)}$ in Massive Scalar Free Field Theory 115

D.1 Preliminaries . . . . . . . . . . . . . . . . . . . . . . 115

D.2 Verification of Condition $L^{(1)} \ldots \ldots \ldots \ldots \ldots \ldots \ldots$

D.3 Verification of Condition $L_{\sharp}^{(1)} \ldots \ldots \ldots \ldots$. . . . . . . . . . 119

E Verification of Condition $N_{h}$ in Scalar Free Field Theorv 123

E.1 Preliminaries . . . . . . . . . . . . . . . . . . . . . . . 123

E.2 Verification of Condition $N_{b} \ldots \ldots \ldots$. . . . . . . . . . . . 124

F Verification of Condition $C_{b}$ in Massive Scalar Free Field Theorv $\quad 131$

F.1 Main Line of Aroument . . . . . . . . . . . . . . . . . . . . . . . . 131

F.2 Proof of Lemma F.1.3 . . . . . . . . . . . . . . . . . . . . . 134

F.3 Some Technical Proofs . . . . . . . . . . . . . . . . . . . . . . . 138

$\begin{array}{lr}\text { Bibliography } & 142\end{array}$

$\begin{array}{lr}\text { Notational Conventions } & 149\end{array}$

\begin{tabular}{ll}
\hline Acknowledgements & 154
\end{tabular} 


\section{Chapter 1}

\section{Introduction}

The understanding of quantum theories in terms of particles has been a fundamental issue for more than four decades. While in the framework of non-relativistic quantum mechanics this problem has been settled for a large class of physically relevant models, the situation is less clear in relativistic quantum field theory (QFT). There emerge new phenomena in this setting, like creation of charged particles in collisions or appearance of clouds of massless excitations accompanying a charged particle ('the infraparticle problem'). Their description goes beyond the well understood setting of groups of unitaries acting on a Hilbert space, where the notions of spectral measure and measure classes provide the natural conceptual basis for the formulation and resolution of the problem of asymptotic completeness. The language of groups of automorphisms acting on the algebra of observables, adequate in the relativistic setting, lacks such detailed spectral concepts.

The present work introduces a decomposition of the algebra of observables into spectral subspaces, whose elements differ in their behavior under translations in space. First, an ergodic theorem for translation automorphisms is established in theories with physical vacuum structure. It allows for a natural definition of the pure-point and continuous subspaces, what opens the door to more detailed spectral analysis: Apart from the counterpart of the absolutely continuous subspace, familiar from quantum mechanics, there appears a new feature - the point-continuous subspace - which carries information about the infrared structure of a theory. It formally belongs to the continuous part, but it is finite dimensional in a large class of models. In particular, it is shown that this subspace is trivial in all theories complying with a condition proposed in this work. This new criterion, which identifies a novel class of particle detectors in the algebra of observables, entails the existence of particles if the theory admits a stress-energy tensor. These results allow for a fresh look at the problem of asymptotic completeness in quantum field theory from the model-independent perspective.

Formulation of natural assumptions for the ergodic theorem mentioned above is the subject of the second part of this work. For this purpose two new phase space conditions are proposed, inspired by the physical behavior of coincidence measurements and by the concept of additivity of energy over isolated subregions, respectively. Among the consequences of these criteria, we obtain the uniqueness of the energetically accessible vacuum states and the convergence of physical states to these vacua under large timelike translations (i.e. relaxation to the vacuum). 


\subsection{Particle Content in Quantum Mechanics. Spectrum of Hamiltonian}

In order to emphasize the relevance of detailed spectral concepts to the problem of particle interpretation, let us consider briefly the familiar case of two-body quantum mechanical scattering: Here the central object is the (relative motion) Hamiltonian $H=H_{0}+V$ which consists of the free part $H_{0}$ and the interaction potential $V$. The Hilbert space $\mathcal{H}$ can be decomposed into the orthogonal subspaces

$$
\mathcal{H}=\mathcal{H}_{\mathrm{pp}} \oplus \mathcal{H}_{\mathrm{ac}} \oplus \mathcal{H}_{\mathrm{sc}}
$$

corresponding to the decomposition of the spectral measure of $H$ into the pure-point, absolutely continuous and singular-continuous parts. By comparing the free dynamics to the interacting dynamics at asymptotic times, one obtains, under suitable assumptions on $V$, the wave operators

$$
W^{ \pm}=\operatorname{s}_{t \rightarrow \pm \infty} e^{i H t} e^{-i H_{0} t} .
$$

One says that the theory has a complete particle interpretation, if $\operatorname{Ran} W^{ \pm}=\mathcal{H}_{\mathrm{ac}}$ and $\mathcal{H}_{\mathrm{sc}}=\{0\}$. Under these conditions every state in the Hilbert space can be uniquely decomposed into the bound states and the scattering states of the Hamiltonian. The first proof of asymptotic completeness in the above framework, for a certain class of short-range potentials, is due to Enss [En78. The argument was later generalized by Sigal and Soffer SiSo87, Graf Gr90 and Dereziński De93 to many body systems interacting with shortrange or long-range forces and it forms today a part of standard textbook material [DG].

\subsection{Wigner's Particle Concept and its Limitations}

To understand the limitations of the above approach to the problem of asymptotic completeness, let us now describe its implementation in QFT. The general framework, based on the algebra of observables $\mathfrak{A}$, is explained in detail in Section [1.6. For the purpose of the present discussion we assume in addition that the action of the whole Poincaré group $P_{+}^{\uparrow}$ is unitarily implemented by a strongly continuous representation $U$ acting on the Hilbert space $\mathcal{H}$ and that there exists a unique (up to phase) vacuum vector $\Omega \in \mathcal{H}$, invariant under the action of $U$. Since the joint spectrum of the generators of translations $(H, \vec{P})$ is contained in the closed forward light-cone $\bar{V}_{+}$, one can define the mass operator $M=\sqrt{H^{2}-\vec{P}^{2}}$.

Following the standard procedure of Wigner Wi39, one unravels the particle content of a theory as follows: First, one finds all the (non-simple) eigenvalues $m$ of the mass operator $M$. Next, one decomposes the corresponding eigenspaces into subspaces $\mathcal{H}^{[m, s]}$ which carry irreducible sub-representations of $U$ characterized by a mass $m$ and a spin $s$. Finally, one forms the direct sum of all such subspaces $\mathcal{H}_{\mathrm{sp}}:=\bigoplus \mathcal{H}^{[m, s]}$ which is called the single-particle subspace of the theory. Scattering theory for massive Wigner particles is well understood by the work of Lehmann, Symanzik and Zimmermann [LSZ55] on the one hand and by Haag, Ruelle, Hepp and Herbst Ha58, Ru62, He65, Her71] on the other. The situation is less clear in theories with long range forces. There collision theory is under complete control only for massless particles, by the work of Buchholz Bu75, Bu77]. The case of massive particles accompanied by massless excitations was treated by the 
present author in Dy05 under a stability assumption introduced by Herbst Her71. The argument is presented in Appendix $\mathrm{A}$ of this Thesis.

The approach of Haag and Ruelle resembles in many respects the quantum-mechanical setting of the previous section: One compares the interacting dynamics governed by the Hamiltonian $H$ to the dynamics of free field theory of mass $m$, for any (non-simple) eigenvalue of the mass operator. There follows the existence of wave operators $W^{ \pm}$which are isometries from the Fock space over the single-particle space $\Gamma\left(\mathcal{H}_{\mathrm{sp}}\right)$ to the physical Hilbert space $\mathcal{H}$

$$
W^{ \pm}: \Gamma\left(\mathcal{H}_{\mathrm{sp}}\right) \rightarrow \mathcal{H}
$$

Thereby, for every configuration of incoming or outgoing particles we can find the corresponding vector in $\mathcal{H}$. If the wave operators are invertible, i.e. every vector in the physical Hilbert space can be interpreted in terms of configurations of incoming and outgoing particles, then we say that the theory is asymptotically complete in the Wigner sense.

The only known class of interacting theories which satisfy this property are the twodimensional models with factorizing $S$-matrices recently constructed by Lechner [Le08]. In particular, no asymptotically complete model exhibiting particle production is known to date. In the thoroughly studied $\lambda\left(\phi^{4}\right)_{2}$ theory only states of energy smaller than $3 m-\varepsilon$

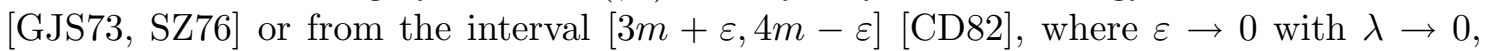
have been shown to have particle interpretation. More importantly, the above variant of asymptotic completeness is bound to fail in many physically relevant situations: Suppose that a pair of charged particles is produced in a collision of neutral particles. Since the masses of the charged particles are not visible in the energy-momentum spectrum of the vacuum sector, the vector $\Psi \in \mathcal{H}$, which corresponds to this process, cannot be interpreted in terms of the Fock space $\Gamma\left(\mathcal{H}_{\mathrm{sp}}\right)$. In the case of massive particles and (string-)localized charges one could try to avoid this difficulty by adjoining the charged sectors, determining the masses of charged particles from the extended energy-momentum spectrum and studying suitably redefined wave operators [BF82]. This strategy fails, however, for electrically charged particles, whose masses are not eigenvalues of $M$, even in the charged sector, due to the presence of Gauss' Law $\mathrm{Bu} 86$. In this case the Wigner concept of a particle does not apply and the approach of Haag and Ruelle is invalidated from the very beginning. The presence of such infraparticles [Sch63] is the main motivation for the search for a more general definition of a particle.

\subsection{Beyond Wigner's Particle Concept. Arveson Spectrum}

The first attempt at an intrinsic characterization of theories describing particles was made by Haag and Swieca HS65. These authors proposed a physically motivated phase space condition which should hold in all theories with reasonable particle interpretation. In fact, a large class of models are known to date to satisfy this criterion BDL90, and a general argument, which corroborates the heuristic reasoning of Haag and Swieca, was found by Bros Br03 in the two-particle situation. Moreover, it was shown by Enss En75] that the Wigner definition of a (massive) particle is equivalent to its geometric characterization as a state which is singly-localized at all times in theories satisfying this phase space condition.

However, as this phase space criterion holds also in some models which do not have particle interpretation, it is too weak to meet the original goal of Haag and Swieca. A 
number of more stringent restrictions on the phase space structure of a theory, formulated in terms of compactness and nuclearity conditions, can be found in the existing literature BP90. They proved very useful in the structural analysis of quantum field theories [BWi86, BJ89, Bu96, Bos05.1, Bos05.2 and in the construction of interacting models [Le08, but have not offered, as yet, many new insights into the particle aspects of the theory. Recently a novel phase space condition, related to additivity of energy over isolated subregions, was proposed by the present author Dy08.1, Dy08.2. Among other physical consequences, it entails relaxation of any state $\omega$ of bounded energy to the vacuum state under large timelike translations, i.e.

$$
\lim _{t \rightarrow \infty} \omega\left(\alpha_{t}(C)\right)=(\Omega \mid C \Omega), \quad C \in \mathfrak{A},
$$

where $\alpha_{t, \vec{x}}(\cdot)=U(t, \vec{x}) \cdot U(t, \vec{x})^{*}$ are the translation automorphisms. The argument, which is given in Chapter 3 of the present work, does not require the assumption of asymptotic completeness in the Wigner sense which was used by Araki and Haag [AH67] in the first proof of relaxation to the vacuum. While relation (1.3.1) does not carry information about the particle content of a theory, we recall that in [AH67] the authors derived an asymptotic expansion of the function $t \rightarrow \omega\left(\alpha_{t}(C)\right)$ as $t \rightarrow \infty$ and demonstrated that the higher-order terms are directly related to the asymptotic particle density. A large part of this analysis was extended beyond the framework of Wigner particles by Buchholz, Porrmann and Stein BPS91] leading to a generalized concept of a particle, encompassing also the case of infraparticles. The remaining part of this section is devoted to a brief sketch and discussion of these developments. (See [MS85, Sp, CFP07, Herd07, for other approaches to the infrared problem).

In order to compensate for the dispersive effects, encoded in equation (1.3.1), one paves the whole space with observables and sums up the results. This amounts to studying the time evolution of the integrals $\int d^{s} x \omega\left(\alpha_{t, \vec{x}}(C)\right)$ which, however, make sense only for suitably chosen $C \in \mathfrak{A}$. In order to introduce a class of admissible particle detectors, we need the mathematical concept of the Arveson spectrum $\mathrm{Ar} 82$, Pe. Postponing the formal definition to Section 1.6. we only recall here that the (local) Arveson spectrum of an element $B \in \mathfrak{A}$ w.r.t. the group of translation automorphisms $\mathbb{R}^{s+1} \ni x \rightarrow \alpha_{x}$, denoted by $\operatorname{Sp}_{B}\left(\alpha_{\mathbb{R}^{s+1}}\right)$, coincides with the energy-momentum transfer of the observable $B$. In fact, let $\Delta \rightarrow P(\Delta)$ be the spectral measure of the energy-momentum operators $(H, \vec{P})$, defined on Borel sets $\Delta \subset \mathbb{R}^{s+1}$. Then there holds

$$
B P(\Delta) \mathcal{H} \subset P\left(\Delta+\operatorname{Sp}_{B}\left(\alpha_{\mathbb{R}^{s+1}}\right)\right) \mathcal{H} .
$$

In view of this relation we say that an operator $B \in \mathfrak{A}$ is energy-decreasing if $\operatorname{Sp}_{B}\left(\alpha_{\mathbb{R}^{s+1}}\right) \cap$ $\bar{V}_{+}=\emptyset$. It is a crucial result due to Buchholz [Bu90] that for any $B \in \mathfrak{A}$ which is energydecreasing and almost loca 1 , and any compact set $\Delta \subset \mathbb{R}^{s+1}$, one can define the integrals

$$
P(\Delta) \int d^{s} x \alpha_{\vec{x}}\left(B^{*} B\right) P(\Delta)
$$

as bounded operators on $\mathcal{H}$. Having compensated for the dispersive effects, one extracts information about the particle content of the theory: For any state $\omega$ of bounded energy one studies the behavior of the following expressions as $t \rightarrow \infty$

$$
\sigma_{\omega}^{(t)}\left(B^{*} B\right)=\int d^{s} x \omega\left(\alpha_{t, \vec{x}}\left(B^{*} B\right)\right)
$$

\footnotetext{
${ }^{1}$ See Section 1.6 for the definition of this concept.
} 
It was shown by Porrmann Po04.1, Po04.2 that the resulting asymptotic functionals $\sigma_{\omega}^{(+)}$, defined on a certain algebra of admissible observables, can be decomposed into pure functionals $\sigma_{\lambda}^{(+)}$, the so called pure particle weights. More precisely, for any $\omega$ there exists a measure $d \mu$ on the space of labels $\lambda$ s.t.

$$
\sigma_{\omega}^{(+)}=\int d \mu(\lambda) \sigma_{\lambda}^{(+)}
$$

To each label $\lambda$ there corresponds a sharp four-momentum $p_{\lambda}$ and a label $\gamma_{\lambda}$ which carries information about the internal degrees of freedom, like spin and charge. Therefore, we can interpret the weights $\sigma_{\lambda}^{(+)}$as plane wave configurations of the particles appearing in the theory. A general algorithm for computation of collision cross-sections of these particles, which does not rely on the existence of charged fields, was developed in BPS91. and tested in asymptotically complete theories of Wigner particles by Stein St89. Also the fundamental problem of the existence of non-trivial asymptotic functionals, which rephrases the question posed by Haag and Swieca HS65, has been settled to date only in this restrictive framework.

Two heuristic arguments of more general nature, addressing the question of existence of particles, were proposed by Buchholz: The first one combines phase space properties of a theory and the time-slice axiom [Bu87]. The second relies on the existence of the stress-energy tensor Bu94. In Section 2.3 of this Thesis we present a rigorous proof of the existence of non-trivial asymptotic functionals which is based on this latter idea and does not rely directly on the Wigner concept of a particle.

Another important issue is the convergence of the asymptotic functional approximants $\sigma_{\omega}^{(t)}$ as $t \rightarrow \infty$. Again, a proof is known only in the context of theories which are asymptotically complete in the Wigner sense AH67. We do not present a solution in this work, but a promising strategy is discussed in Chapter 4 . The simpler problem of relaxation to the vacuum, (cf. relation (1.3.1) above), which is settled in this Thesis under physically meaningful conditions, should provide a guidance towards a more general proof of the convergence of $\sigma_{\omega}^{(t)}$.

Very little is known about asymptotic completeness in this general framework. A possible formulation was proposed in Bu94: It should be possible to determine the energy and momentum of any physical state $\omega$, knowing its particle content from relation (1.3.5). More precisely, there should hold

$$
\omega(P)=\int d \mu(\lambda) p_{\lambda}
$$

where the four-momenta $p_{\lambda}$ label the pure particle weights $\sigma_{\lambda}^{(+)}$and a similar relation should hold for other conserved quantities which characterize particles, like spin, charges etc. It is plausible that relation (1.3.6) holds in models admitting a stress-energy tensor, but we are far from an actual proof. Additional assumptions, which may be useful to settle this issue, are discussed in Section 2.3

It is evident from the above discussion that the problem of particle interpretation in quantum field theory is rather poorly understood in comparison to the quantummechanical case considered in Section 1.1. We see the origin of this disproportion in the absence of adequate mathematical structures on the side of QFT: Here the natural language for the description of particle aspects is that of the group of translation automorphisms $\mathbb{R}^{s+1} \ni x \rightarrow \alpha_{x}$ acting on the $C^{*}$-algebra $\mathfrak{A}$. The Hamiltonian, central for 
the quantum-mechanical scattering, is replaced by the generator of time translation automorphisms, whose spectrum coincides with the (global) Arveson spectrum of $\alpha_{t}$ [Ev76], defined by formula (1.4.2) below. After the spectral theory of automorphisms was systematized by Arveson Ar82, it became clear that several important notions, familiar from the quantum-mechanical setting, do not have counterparts in this more general context. These include the concept of spectral measure and measure classes consisting of pure-point, Lebesgue absolutely continuous and singular continuous parts, and the corresponding decomposition (1.1.1) of the Hilbert space into spectral subspaces. As we have seen in Section 1.1. these notions are crucial for the formulation and resolution of the problem of asymptotic completeness in quantum mechanics. It is therefore quite certain that the lack of counterparts on the side of automorphism groups impedes the study of the particle aspects in QFT. Various steps towards the development of such more detailed theory of the Arveson spectrum, which can be found in the literature, are discussed in the next section.

\subsection{Detailed Theory of Arveson Spectrum in Literature}

For a group of unitaries $\mathbb{R}^{s} \ni \vec{x} \rightarrow U(\vec{x})$, acting on a Hilbert space, there holds $U(\vec{x}) \Psi=\int e^{-i \vec{p} \vec{x}} d P(\vec{p}) \Psi$, where $\Psi \in \mathcal{H}$ and $d P$ is the spectral measure. Hence the natural counterpart of the spectral measure in the Arveson theory are the Fourier transforms of the functions $\mathbb{R}^{s} \ni \vec{x} \rightarrow \alpha_{\vec{x}}(A)$, where $A \in \mathfrak{A}$. As the support of the resulting distribution $\vec{p} \rightarrow \widetilde{A}(\vec{p})$ coincides with the Arveson spectrum of $A$, a more detailed spectral theory should describe also its regularity properties.

Several results in this direction can be found in the existing literature. On the physics side, the distributions $\vec{p} \rightarrow \omega(\widetilde{A}(\vec{p}))$, where $A$ is a local operator and $\omega$ a state of bounded energy, were studied by Buchholz Bu90. It was shown that in any local, relativistic QFT they coincide with square-integrable functions apart from a possible singularity at zero. This result, stated precisely in Theorem 1.6.2 below, was instrumental for the development of the Haag-Ruelle scattering theory in the presence of massless particles Dy05, treated in Appendix $\mathrm{A}$ of this work. It also provides a basis for the spectral decomposition of the algebra of local observables, which we construct in Chapter 2

Such Fourier coefficients $\widetilde{A}(\vec{p})$ appear also as a tool in the mathematical literature related to the Rieffel project of extending the notions of proper action and orbit space from the setting of group actions on locally compact spaces to the context of $C^{*}$-dynamical systems $\left(\mathfrak{A}, \mathbb{R}^{s}, \alpha\right)$ [Ri90, Me01]. A number of properties of $\widetilde{A}(\vec{p})$, familiar from classical harmonic analysis, like the Fourier inversion formula, have been proven by Exel in Ex99, Ex00. Unfortunately, we cannot use these results here as they rely on the assumption of unconditional integrability, which requires that the net

$$
\left\{\int_{K} d^{s} x \alpha_{\vec{x}}(A)\right\}_{K},
$$

indexed by compact subsets $K \subset \mathbb{R}^{s}$, converges in the norm topology of $\mathfrak{A}$ as $K \nearrow \mathbb{R}^{s}$. This is not quite compatible with QFT, where we interpret $A \in \mathfrak{A}$ as a particle detector, since for any compact region $K$ one can prepare a physical state which gives large measurement responses in the complement of $K$. In fact, the integral (1.3.3) was only shown to converge in the strong operator topology of $B(\mathcal{H})$ [Bu90]. 
The above two approaches are distinguished by the fact that they exploit the algebraic structure of $\mathfrak{A}$. The more general framework of groups of isometries $\mathbb{R} \ni t \rightarrow \alpha_{t}$ acting on a Banach space $\mathfrak{A}$ attracted more attention. This direction of research relies on the equality of the (global) Arveson spectrum, given by

$$
\operatorname{Sp} \alpha_{\mathbb{R}}=\overline{\bigcup_{A \in \mathfrak{A}} \operatorname{Sp}_{A} \alpha_{\mathbb{R}}}
$$

and the operator theoretic spectrum of the generator $D=\left.\frac{1}{i} \frac{d}{d t} \alpha_{t}\right|_{t=0}$ which was established by Evans [Ev76] and independently by Longo [Lo77. Related results, known as the spectral mapping theorems, were obtained for the global Arveson spectrum [C073, DLZ81, Ar82, [Ne92] and for the local one [Ne98, LN], (see [Ar82, LN] for reviews). As for the more detailed spectral theory, the point spectrum of $D$ is the best understood one. A thorough analysis of the discrete spectrum, including the mini-max principle, existence and uniqueness theorems for the ground state and the Rayleigh-Ritz technique, was performed by Jorgensen Jo82. Inspired by the Stone formula [RS1, this author proposes a suitable limit of resolvents of the generator $D$ to be the counterpart for the spectral measure. This technique has applications also beyond the point spectrum Jo92: Using classical results of Fourier analysis, Jorgensen derives integrability and decay properties of the functions $t \rightarrow \omega\left(\alpha_{t} A\right)$, where $A \in \mathfrak{A}, \omega \in \mathfrak{A}^{*}$, from regularity assumptions on these resolvents. However, such assumptions do not have a clear physical meaning and are difficult to verify in the case of translation automorphisms in QFT, so we do not pursue this approach here.

The subject of Cauchy problems in the theory of differential equations AB97 includes an interesting line of developments which bears some similarity to the present work: Assume that the spectrum of $D$ is countable. Then, under some additional conditions, the eigenvectors of $D$ span a norm dense subspace in $\mathfrak{A}[\mathrm{Ba78}$, Hu99]. In this case the representation $\alpha$ is called almost periodic, as all its orbits $t \rightarrow \alpha_{t}(A), A \in \mathfrak{A}$, belong to the class $A P(\mathbb{R}, \mathfrak{A})$ of almost periodic functions with values in $\mathfrak{A}$. More abstractly, $u \in A P(\mathbb{R}, \mathfrak{A})$ if and only if the set $S(u)=\{u(\cdot+t) \mid t \in \mathbb{R}\}$ of the translates of $u$ is relatively norm compact in the Banach space $B U C(\mathbb{R}, \mathfrak{A})$ of bounded, uniformly continuous functions AB97, Ph93. Replacing in the above definitions the real line $\mathbb{R}$ with the positive half-line $\mathbb{R}_{+}$, we obtain the set $S(u)_{+}$and the class $A A P\left(\mathbb{R}_{+}, \mathfrak{A}\right)$ of asymptotically almost periodic functions which is of interest in the theory of $C_{0}$-semigroups $\mathbb{R}_{+} \ni t \rightarrow \alpha_{t}$ acting on Banach spaces. This class has the decomposition [RS90, AB99, BPh90, AB88,

$$
A A P\left(\mathbb{R}_{+}, \mathfrak{A}\right)=\left.A P(\mathbb{R}, \mathfrak{A})\right|_{\mathbb{R}_{+}} \oplus C_{0}\left(\mathbb{R}_{+}, \mathfrak{A}\right),
$$

where $C_{0}\left(\mathbb{R}_{+}, \mathfrak{A}\right)$ is the space of continuous, functions which tend to zero in norm as $t \rightarrow \infty$. A larger class $W\left(\mathbb{R}_{+}, \mathfrak{A}\right)$ of Eberlain weakly almost periodic functions is characterized by the condition that the set $S(u)_{+}$is relatively weakly compact [RS90, RS92]. The weak counterpart of $C_{0}\left(\mathbb{R}_{+}, \mathfrak{A}\right)$ is the space $W_{0}\left(\mathbb{R}_{+}, \mathfrak{A}\right)$ of such functions $u$ from $W\left(\mathbb{R}_{+}, \mathfrak{A}\right)$, that zero belongs to the weak closure of $S(u)_{+}$. Again, we have a decomposition [RS90]

$$
W\left(\mathbb{R}_{+}, \mathfrak{A}\right)=\left.A P(\mathbb{R}, \mathfrak{A})\right|_{\mathbb{R}_{+}} \oplus W_{0}\left(\mathbb{R}_{+}, \mathfrak{A}\right) .
$$

All the spaces introduced above consist of orbits of the semigroup of translations given by $\left(\alpha_{t} u\right)\left(t^{\prime}\right)=u\left(t+t^{\prime}\right)$ acting on $B U C(\mathbb{R}, \mathfrak{A})$. Therefore, relations (1.4.3), (1.4.4) can 
be interpreted as spectral decompositions of the corresponding generator $D$. However, in contrast to the Hilbert space case (cf. relation (1.1.1)), these decompositions do not involve the whole underlying space $B U C(\mathbb{R}, \mathfrak{A})$, but only cover some small neighborhoods of the pure-point part $\left.A P(\mathbb{R}, \mathfrak{A})\right|_{\mathbb{R}_{+}}$. Moreover, they consist only of the pure-point subspace and its complement which can be understood as a candidate for the continuous subspace. There does not seem to appear any further decomposition of these continuous subspaces in the literature. Finally, and most importantly, this approach is appropriate for the study of semigroups of operators rather than groups of isometries we are interested in here. In particular, the space $C_{0}\left(\mathbb{R}_{+}, \mathfrak{A}\right)$ does not contain orbits of any group of isometries.

\subsection{Overview of this Work}

In Chapter 1 we gave an overview of scattering theory in quantum mechanics and quantum field theory placing emphasis on the role of spectral analysis. The remaining part of this chapter treats the framework of algebraic quantum field theory as well as definitions and results which are particularly useful in the later part of this Thesis.

In Chapter 2] we motivate and develop a detailed spectral theory of space translation automorphisms $\mathbb{R}^{s} \ni \vec{x} \rightarrow \alpha_{\vec{x}}$ acting on the algebra of local observables $\hat{\mathfrak{A}}$. We obtain the decomposition

$$
\hat{\mathfrak{A}}=\hat{\mathfrak{A}}_{\mathrm{pp}} \oplus \hat{\mathfrak{A}}_{\mathrm{pc}} \oplus \hat{\mathfrak{A}}_{\mathrm{ac}} .
$$

Apart from the pure-point and absolutely continuous parts, familiar from the Hilbert space context, there appears a new subspace which we call point-continuous. It formally belongs to the continuous part of the spectrum, but it is finite dimensional in a large family of models, complying with Condition $L^{(2)}$ stated in Section 2.2. This subspace carries information about the infrared behavior of the theory which can be assessed with the help of a new quantity which we call the infrared order of an operator. We provide examples of theories with non-trivial and trivial point-continuous subspaces and compute the infrared orders of their elements. Triviality of the point-continuous subspace is expected to hold in purely massive theories and we formulate a general condition $L^{(1)}$, stated in Section 2.3 , which entails this spectral property. A quantitative variant of this new criterion guarantees the existence of non-trivial asymptotic functionals (describing particles) in theories admitting a stress-energy tensor.

In Chapter 3 we study the uniqueness of the decomposition of $\hat{\mathfrak{A}}$ into the pure-point and continuous parts. A variant of the ergodic theorem for translation automorphisms in QFT, stated as Theorem 2.2.2 below, reduces this issue to the problem of the uniqueness of the energetically accessible vacuum state. In Section 3.3 we introduce a phase space condition $C_{\mathrm{b}}$, of algebraic nature, which entails this property and has a number of other physically interesting consequences: The vacuum state is pure and appears, in particular, as a limit of physical states under large spacelike or timelike translations in Lorentz covariant theories. Moreover, it is approximated by states of increasingly sharp energymomentum values, in accordance with the uncertainty principle. This new condition has a clear physical interpretation in terms of coincidence arrangements of local observables, but its consistency with the basic postulates has been verified only in the realm of massive theories. Therefore, in Section 3.4 we introduce another phase space condition $C_{\mathfrak{b}}$, involving only the Banach space structure of $\mathfrak{A}$, which can be verified both in massive and massless models. We motivate this condition by the physical principle of additivity of 
energy over isolated regions and show that it has all the physical consequences mentioned above (apart from the purity of the vacuum). We also demonstrate that it can be derived from an auxiliary nuclearity condition $N_{\sharp}$, introduced in Section 3.5 which is better suited for the study of concrete examples.

In Chapter 4 we summarize our results and consider some open problems from a new perspective. In particular, we discuss in detail the problem of convergence of the asymptotic functional approximants.

The main part of this Thesis is accompanied by six appendices: Appendix $\mathrm{A}$ which develops the Haag-Ruelle scattering theory in the presence of massless particles, complements our discussion of collision theory in Section [1.2. Appendix $\mathbb{B}$ summarizes the known results on the phase space structure of scalar free field theory. It provides the basis for the material presented in the remaining appendices. In Appendices $[\mathrm{C}$ and $\mathrm{D}$ we establish Conditions $L^{(2)}$ and $L^{(1)}$, respectively, in models of non-interacting particles. Appendices $\left[\mathrm{E}\right.$ and $\mathrm{F}$ are devoted to verification of Conditions $N_{\bullet}$ and $C_{\mathrm{b}}$ in such models. Notably, Conditions $L^{(2)}$ and $N_{\sharp}$ hold both in the massive and the massless case.

The first and last section of Chapter 3 was published in Dy08.1. Appendix A essentially coincides with Dy05. The argument from Appendix $\mathrm{E}$ appeared (for the massless case) in Dy08.2.

\subsection{Technical Background}

We adopt here the standard Haag-Kastler framework of algebraic quantum field theory $\mathrm{Ha}, \mathrm{Ar}$. Let $\mathcal{H}$ be an infinitely dimensional Hilbert space and let $\mathfrak{A}=\{\mathfrak{A}(\mathcal{O}) \subset$ $\left.B(\mathcal{H}) \mid \mathcal{O} \subset \mathbb{R}^{s+1}\right\}$ be a net of local von Neumann algebras attached to open bounded regions $\mathcal{O}$ of the Minkowski spacetime. We denote by $\hat{\mathfrak{A}}=\bigcup_{\mathcal{O} \subset \mathbb{R}^{s+1}} \mathfrak{A}(\mathcal{O})$ the $*$-algebra of local observables and its norm closure $\mathfrak{A}$ (denoted by the same symbol as the net) is called the global $C^{*}$-algebra of observables. Finally, let $\alpha$ be a representation of the Poincaré group $P_{+}^{\uparrow}=\mathbb{R}^{s+1} \rtimes L_{+}^{\uparrow}$ in the group of automorphisms of $\mathfrak{A}$ s.t. for any $A \in \hat{\mathfrak{A}}$ the function $P_{+}^{\uparrow} \ni(x, \Lambda) \rightarrow \alpha_{(x, \Lambda)}(A)$ is continuous in the strong operator topology of $B(\mathcal{H})$. The triple $(\mathfrak{A}, \alpha, \mathcal{H})$ is called an (algebraic) quantum field theory if it satisfies the following conditions:

1. Isotony: If $\mathcal{O}_{1} \subset \mathcal{O}_{2}$ then $\mathfrak{A}\left(\mathcal{O}_{1}\right) \subset \mathfrak{A}\left(\mathcal{O}_{2}\right)$.

2. Locality: If $\mathcal{O}_{1}$ and $\mathcal{O}_{2}$ are spacelike separated, then $\mathfrak{A}\left(\mathcal{O}_{1}\right) \subset \mathfrak{A}\left(\mathcal{O}_{2}\right)^{\prime}$, where the prime denotes the commutant in $B(\mathcal{H})$.

3. Irreducibility: The global $C^{*}$-algebra $\mathfrak{A}$ acts irreducibly on the Hilbert space $\mathcal{H}$.

4. Covariance: The representation $\alpha$ of $P_{+}^{\uparrow}$ acts geometrically on the net i.e.

$$
\alpha_{(x, \Lambda)} \mathfrak{A}(\mathcal{O})=\mathfrak{A}(\Lambda \mathcal{O}+x) .
$$

5. Spectrum condition: There exists a strongly continuous unitary representation of translations $\mathbb{R}^{s+1} \ni x \rightarrow U(x)$ acting on the Hilbert space $\mathcal{H}$ which implements the translation automorphisms i.e. $\alpha_{x}(\cdot)=U(x) \cdot U(x)^{*}$. The joint spectrum of the infinitesimal generators of translations $\left(H, P_{1}, \ldots, P_{s}\right)$, corresponding to this unitary representation, is contained in the closed future light cone $\bar{V}_{+}=\left\{\left(p^{0}, \vec{p}\right) \in\right.$ $\left.\mathbb{R}^{s+1}\left|p^{0} \geq\right| \vec{p} \mid\right\}$. 
We will occasionally consider sub-theories of a given theory: by a sub-theory of $(\mathfrak{A}, \alpha, \mathcal{H})$ we mean a triple $(\mathfrak{B}, \alpha, \mathcal{H})$ satisfying the above assumptions and s.t. $\mathfrak{B}(\mathcal{O}) \subset \mathfrak{A}(\mathcal{O})$ for any open, bounded region $\mathcal{O}$. At times we use the notation $A(x)=\alpha_{x}(A), \varphi_{x}=\alpha_{x}^{*} \varphi$ for translated observables $A \in \mathfrak{A}$ and functionals $\varphi \in \mathfrak{A}^{*}$. For any Banach space $X$, we denote by $X_{1}$ the unit ball in $X$. Other definitions and results which are central in the later part of this Thesis are listed below:

- Vectors of bounded energy. We denote by $P_{E}$ be the spectral projection of the Hamiltonian $H$ on vectors of energy bounded by $E$ and define the dense subspace $D_{B}=\bigcup_{E \geq 0} P_{E} \mathcal{H}$ of vectors of bounded energy.

- Functionals of bounded energy. It follows from the irreducibility property above that the space of normal states on $\mathfrak{A}$ coincides with $B(\mathcal{H})_{*}$ which can be identified with the space $\mathcal{T}$ of trace-class operators on $\mathcal{H}$. We introduce the subspace $\mathcal{T}_{E}=$ $P_{E} \mathcal{T} P_{E}$ of normal functionals of restricted energy. We denote the cone of positive functionals from $\mathcal{T}_{E}$ by $\mathcal{T}_{E}^{+}$and the subset of states from $\mathcal{T}_{E}^{+}$by $S_{E}$. The states from $\mathfrak{A}^{*}$ which belong to the weak ${ }^{*}$ closure of $\mathcal{T}_{E, 1}$ for some $E \geq 0$ will be called the energetically accessible states.

It is a well known fact that any normal, self-adjoint functional on a von Neumann algebra can be expressed as a difference of two normal, positive functionals which are mutually orthogonal $\left[\mathrm{Sa}\right.$. It follows that any $\varphi \in \mathcal{T}_{E, 1}$ can be decomposed as

$$
\varphi=\varphi_{\operatorname{Re}}^{+}-\varphi_{\operatorname{Re}}^{-}+i\left(\varphi_{\operatorname{Im}}^{+}-\varphi_{\operatorname{Im}}^{-}\right),
$$

where $\varphi_{\operatorname{Re}}^{ \pm}, \varphi_{\operatorname{Im}}^{ \pm}$are elements of $\mathcal{T}_{E, 1}^{+}$.

- Almost local observables. A typical region of spacetime to be used in the sequel is the double cone $\mathcal{O}(r)=\left\{\left(x^{0}, \vec{x}\right) \in \mathbb{R}^{s+1}|| x^{0}|+| \vec{x} \mid<r\right\}, r>0$, whose base is the $s$-dimensional ball $\mathcal{O}_{r}=\left\{\vec{x} \in \mathbb{R}^{s}|| \vec{x} \mid<r\right\}$ of radius $r$ centered at the origin. We say that $A \in \mathfrak{A}$ is almost local, if there exists a net of local operators $\left\{A_{r} \in \mathfrak{A}(\mathcal{O}(r)) \mid r>0\right\}$ such that

$$
\lim _{r \rightarrow \infty} r^{k}\left\|A-A_{r}\right\|=0
$$

for any $k \in \mathbb{N}_{0}$.

- Arveson spectrum w.r.t. spacetime translations. Given an observable $B \in \mathfrak{A}$, its (local) Arveson spectrum w.r.t. the group of translation automorphisms $\mathbb{R}^{s+1} \ni$ $x \rightarrow \alpha_{x}$ is denoted by $\operatorname{Sp}_{B}\left(\alpha_{\mathbb{R}^{s+1}}\right)$ and defined as the support of the operator-valued distribution

$$
\widetilde{B}(p)=\frac{1}{(2 \pi)^{\frac{s+1}{2}}} \int d^{s+1} x e^{-i p \cdot x} \alpha_{x}(B) .
$$

We say that $B$ is energy-decreasing if $\operatorname{Sp}_{B}\left(\alpha_{\mathbb{R}^{s+1}}\right) \cap \bar{V}_{+}=\emptyset$. There holds the following important result:

Theorem 1.6.1. [Bu90] Let $B \in \mathfrak{A}$ be energy-decreasing and almost local. Let $X \subset \mathbb{R}^{s+1}$ be some subspace spanned by spacelike vectors and $d x$ be a translationally 
invariant measure on $X$. Then, for any compact subset $K \subset X$ and any $E \geq 0$, there holds the bound

$$
\left\|P_{E} \int_{K} d x \alpha_{x}\left(B^{*} B\right) P_{E}\right\| \leq c_{E}
$$

where the constant $c_{E}$ is independent of $K$.

The statement holds in particular for $X=\mathbb{R}^{s}$ or $X=\{\lambda \hat{e} \mid \lambda \in \mathbb{R}\}$, where $\hat{e}$ is some spacelike unit vector.

- Arveson spectrum w.r.t. space translations. Given an observable $B \in \mathfrak{A}$, its Arveson spectrum w.r.t. the group of space translation automorphisms $\mathbb{R}^{s} \ni \vec{x} \rightarrow \alpha_{\vec{x}}$ is denoted by $\operatorname{Sp}_{A}\left(\alpha \mathbb{R}^{s}\right)$ and defined as the support of the operator-valued distribution

$$
\widetilde{A}(\vec{p})=\frac{1}{(2 \pi)^{\frac{s}{2}}} \int d^{s} x e^{i \vec{p} \vec{x}} \alpha_{\vec{x}}(A) .
$$

The following result is central for our investigations in Chapter 2

Theorem 1.6.2. Bu90] For any $E \geq 0, A \in \hat{\mathfrak{A}}$ and $\varepsilon>0$ there holds the bound

$$
\sup _{\varphi \in \mathcal{T}_{E, 1}} \int d^{s} p|\vec{p}|^{s+1+\varepsilon}|\varphi(\widetilde{A}(\vec{p}))|^{2}<\infty
$$

- Vacuum states. We say that $\omega_{0} \in \mathfrak{A}^{*}$ is a vacuum state if it satisfies the following conditions

(a) $\alpha_{x}^{*} \omega_{0}=\omega_{0}$ for any $x \in \mathbb{R}^{s+1}$.

(b) $\omega_{0}$ is locally normal i.e. $\left.\omega_{0}\right|_{\mathfrak{A}(\mathcal{O})}$ belongs to $\mathfrak{A}(\mathcal{O})_{*}$ for any open, bounded region $\mathcal{O}$.

(c) In the GNS-representation of $\mathfrak{A}$ induced by $\omega_{0}$ there holds the spectrum condition (given by property 5 above).

We note that the local normality property (b) and the fact that local observables form a norm dense subspace in $\mathfrak{A}$ ensure the continuity of the functions $\mathbb{R}^{s+1} \ni$ $x \rightarrow \omega_{0}(A B(x))$, for any $A, B \in \mathfrak{A}$. This entails the existence of the generators of translations $\left(H_{\omega_{0}}, \vec{P}_{\omega_{0}}\right)$ in the GNS-representation induced by $\omega_{0}$. 


\section{Chapter 2}

\section{Spectral Decomposition and Existence of Particles}

The goal of this chapter is twofold: First, to formulate general conditions on the local net of observables which imply that the particle content of a theory is non-trivial. More precisely, to assure that the limit points as $t \rightarrow \infty$ of the integrals

$$
\int d^{s} x \omega\left(\alpha_{t, \vec{x}}(C)\right)
$$

are finite and different from zero for some observables $C \in \mathfrak{A}$ and physical states $\omega$. Following the heuristic reasoning from Bu94, our strategy is to link the almost local observables $C$ to the $(0,0)$-component of the stress-energy tensor $T^{00}$, whose integral over the whole space is a constant of motion. The fact that $T^{00}$ is a local quantity motivates the second aim of this chapter, namely to understand the behavior of local observables under translations in space.

As a first orientation we consider in Section 2.1 the quantum mechanical case of space translations $\vec{x} \rightarrow U(\vec{x})$ acting unitarily on a Hilbert space. With the help of the ergodic theorem we formulate the decomposition of the Hilbert space into the respective pure-point and continuous subspaces in a way which facilitates its generalization to the Banach space setting. Proceeding to the more detailed spectral analysis, we show that the generic property of the functions $\vec{x} \rightarrow(\Phi \mid U(\vec{x}) \Psi)$, where the wavefunction of $\Psi$ is localized in space, is not integrability, required in (2.0.1), but the weaker property of square-integrability.

In Section 2.2 we turn our attention to the case of space translation automorphisms $\vec{x} \rightarrow \alpha_{\vec{x}}$ acting on the algebra of local observables $\hat{\mathfrak{A}}$ in QFT. We show that in theories with decent vacuum structure, as described by Condition $V$ stated below, there holds a counterpart of the ergodic theorem. It offers a natural decomposition of $\hat{\mathfrak{A}}$ into the pure-point and continuous parts. The square-integrability of the functions $\vec{x} \rightarrow \omega\left(\alpha_{\vec{x}}(A)\right)$, where $A$ is a local operator, provides a meaningful definition of the absolutely continuous subspace $\hat{\mathfrak{A}}_{\mathrm{ac}}$ for space translations in QFT. In the present case, however, square-integrability may fail, due to singularities at zero momentum transfer, exhibited by some infrared-sensitive observables. A new concept of the infrared order of an operator is introduced to quantify this effect. Such operators typically span a finite-dimensional subspace which we call the point-continuous subspace $\hat{\mathfrak{A}}_{\mathrm{pc}}$. It does not have a quantum mechanical counterpart and carries information about the infrared structure of a theory. Thus we arrive at the 
decomposition

$$
\hat{\mathfrak{A}}=\hat{\mathfrak{A}}_{\mathrm{pp}} \oplus \hat{\mathfrak{A}}_{\mathrm{pc}} \oplus \hat{\mathfrak{A}}_{\mathrm{ac}}
$$

which is studied in examples in Subsection 2.2.3. The more technical part of this discussion is given in Appendix [C]

With integrals (2.0.1) in our main focus, it is certainly of interest to identify theories with regular infrared behavior. Therefore, in Section 2.3. we formulate a condition which assures that the point-continuous subspace is trivial. At the same time it identifies a class of particle detectors which are sufficiently close to the stress-energy tensor, to conclude that the particle content is non-trivial. These results demonstrate the interplay between the spectral aspects and the particle structures in QFT. (The condition is verified in a model of non-interacting massive particles in Appendix D).

\subsection{Space Translations in Quantum Mechanics}

In this section we revisit the well known spectral theory of strongly continuous unitary representations of translations $\mathbb{R}^{s} \ni \vec{x} \rightarrow U(\vec{x})$ acting on a Hilbert space $\mathcal{H}$. Our goal is to motivate its generalization to the context of translation automorphisms acting on the algebra of observables, which we undertake in the next section.

The pure-point subspace is spanned by the joint eigenvectors of the generators of translations i.e.

$$
\mathcal{H}_{\mathrm{pp}}=\operatorname{Span}\left\{\Psi \in \mathcal{H} \mid U(\vec{x}) \Psi=e^{-i \vec{p} \vec{x}} \Psi \text {, for some } \vec{p} \in \mathbb{R}^{s} \text { and all } \vec{x} \in \mathbb{R}^{s}\right\} .
$$

The continuous subspace $\mathcal{H}_{\mathrm{c}}$ is simply the orthogonal complement of $\mathcal{H}_{\mathrm{pp}}$ in $\mathcal{H}$. However, it is convenient for our purposes to have a characterization which does not rely directly on the concept of orthogonality, specific to Hilbert spaces. Therefore, we recall that due to the ergodic theorem [RS1, the spectral projection $P_{\{\vec{p}\}}$ on a point $\vec{p}$ in the joint spectrum of the generators of translations is given by

$$
P_{\{\vec{p}\}}=\operatorname{s}_{K \nearrow \mathbb{R}^{s}} \frac{1}{|K|} \int_{K} d^{s} x U(\vec{x}) e^{i \vec{p} \vec{x}},
$$

where the limit is taken w.r.t. to an increasing net of compact sets $K \subset \mathbb{R}^{s}$ ordered by inclusion. Now the continuous subspace is determined as follows

$$
\mathcal{H}_{\mathrm{c}}=\bigcap_{\vec{p} \in \mathbb{R}^{s}} \operatorname{ker} P_{\{\vec{p}\}} \cdot
$$

This subspace can be further decomposed into the absolutely continuous and singular continuous parts $\mathcal{H}_{\mathrm{c}}=\mathcal{H}_{\mathrm{ac}} \oplus \mathcal{H}_{\mathrm{sc}}$ and we note the following simple criterion which implies that a vector belongs to the absolutely continuous subspace.

Proposition 2.1.1. Suppose that the function $\mathbb{R}^{s} \ni \vec{x} \rightarrow(\Psi \mid U(\vec{x}) \Psi)$ is square-integrable for some $\Psi \in \mathcal{H}$. Then $\Psi \in \mathcal{H}_{\mathrm{ac}}$.

This statement follows from the Plancherel theorem and the fact that any square-integrable function is locally integrable.

In the next section we propose a similar square-integrability condition as a characterization of the 'absolutely continuous' subspace of the algebra of observables. In order 
to motivate such a restrictive definition, we consider now briefly a concrete unitary representation of translations $\mathbb{R}^{s} \ni \vec{x} \rightarrow U(\vec{x})$ acting on the Hilbert space $L^{2}\left(\mathbb{R}^{s}, d^{s} x\right)$ as follows

$$
(U(\vec{x}) \Psi)(\vec{y})=\Psi(\vec{y}-\vec{x}), \quad \Psi \in L^{2}\left(\mathbb{R}^{s}, d^{s} x\right) .
$$

In this setting a natural analogue of a local observable is a vector $\Psi \in L^{2}\left(\mathbb{R}^{s}, d^{s} x\right)$ which has (a representative with) a support in an open bounded region. In the next lemma we show that these vectors satisfy the square-integrability condition stated in Proposition 2.1.1

Lemma 2.1.2. Let $\Phi, \Psi \in L^{2}\left(\mathbb{R}^{s}, d^{s} x\right)$. Suppose that $\Psi$ has (a representative with) a support in an open, bounded region $O \subset \mathbb{R}^{s}$. Then there holds

$$
\int d^{s} x|(\Phi \mid U(\vec{x}) \Psi)|^{2} \leq|O|\|\Phi\|^{2}\|\Psi\|^{2},
$$

where $|O|$ is the volume of the region $O$.

Proof. Using the Plancherel theorem we obtain

$$
\int d^{s} x|(\Phi \mid U(\vec{x}) \Psi)|^{2}=(2 \pi)^{s} \int d^{s} p|\widetilde{\Phi}(\vec{p})|^{2}|\widetilde{\Psi}(\vec{p})|^{2} .
$$

The support property of $\Psi$ gives

$$
|\widetilde{\Psi}(\vec{p})|^{2} \leq(2 \pi)^{-s}\left(\int d^{s} x|\Psi(\vec{x})|\right)^{2} \leq(2 \pi)^{-s}|O|\|\Psi\|^{2},
$$

what concludes the proof.

More importantly, the square-integrability of the functions $\mathbb{R}^{s} \ni \vec{x} \rightarrow(\Phi \mid U(\vec{x}) \Psi)$, proven in the above lemma, cannot be improved to integrability with any lower power. In fact:

Lemma 2.1.3. For any $0<k<2$ there exist $\Phi, \Psi \in L^{2}\left(\mathbb{R}^{s}, d^{s} x\right)$ s.t. $\Psi$ has (a representative with) a support in an open, bounded region $O$ and

$$
\int d^{s} x|(\Phi \mid U(\vec{x}) \Psi)|^{k}=\infty .
$$

Proof. Let $O$ be the ball of radius $R>0$ centered at the origin. Let $\chi \in C_{0}^{\infty}\left(\mathbb{R}^{s}\right)$ be a positive function, s.t. $\chi(\vec{x})=\chi(-\vec{x})$, supported in this ball. We set for some $0<\delta<s$

$$
\begin{aligned}
\tilde{\Phi}(\vec{p}) & :=\frac{\tilde{\chi}(\vec{p})}{|\vec{p}|^{\frac{s-\delta}{2}}}, \\
\tilde{\Psi}(\vec{p}) & :=\tilde{\chi}(\vec{p}) .
\end{aligned}
$$

Making use of the fact that the Fourier transform of $\vec{p} \rightarrow|\vec{p}|^{-\frac{s-\delta}{2}}$ equals $\vec{x} \rightarrow c_{\delta}|\vec{x}|^{-\frac{s+\delta}{2}}>0$, where $c_{\delta}=2^{\frac{\delta}{2}} \frac{\Gamma\left(\frac{s+\delta}{4}\right)}{\Gamma\left(\frac{s-\delta}{4}\right)}$, we obtain

$$
\begin{aligned}
(\Phi \mid U(\vec{x}) \Psi) & =(2 \pi)^{-\frac{s}{2}} \int d^{s} y \frac{c_{\delta}}{|\vec{y}+\vec{x}|^{\frac{s+\delta}{2}}} \int d^{s} z \chi(\vec{z}) \chi(\vec{z}-\vec{y}) \\
& \geq(2 \pi)^{-\frac{s}{2}} \frac{c_{\delta}}{(|\vec{x}|+2 R)^{\frac{s+\delta}{2}}}\left(\int d^{s} y \chi(\vec{y})\right)^{2} .
\end{aligned}
$$


Consequently, for $0<k<2$ and sufficiently small $\delta>0$, integral (2.1.8) diverges.

Summing up, the square-integrability of the functions

$$
\mathbb{R}^{s} \ni \vec{x} \rightarrow(\Phi \mid U(\vec{x}) \Psi),
$$

uniformly in $\Phi \in L^{2}\left(\mathbb{R}^{s}, d^{s} x\right),\|\Phi\| \leq 1$, is the best possible generic feature of vectors $\Psi$, compactly supported in configuration space. Turning our attention to quantum field theory, we recall that such vectors are our analogues of local observables $A \in \hat{\mathfrak{A}}$. Furthermore, vectors $\Phi$ correspond to normal functionals of bounded energy $\varphi \in \mathcal{T}_{E}$ and the group of unitaries $\mathbb{R}^{s} \ni \vec{x} \rightarrow U(\vec{x})$ is the counterpart of the group of translation automorphisms $\mathbb{R}^{s} \ni \vec{x} \rightarrow \alpha_{\vec{x}}$. Thereby the transition amplitudes (2.1.13) between $\Phi$ and the translates of $\Psi$ provide a toy model for the expectation values of translates of local operators on states of bounded energy:

$$
\mathbb{R}^{s} \ni \vec{x} \rightarrow \varphi\left(\alpha_{\vec{x}}(A)\right) .
$$

These functions are the main subject of the remaining part of this chapter.

\subsection{Space Translations in Quantum Field Theory. Spectral Decomposition}

In this section we construct the spectral decomposition of the $*$-algebra of local operators $\hat{\mathfrak{A}}=\bigcup_{\mathcal{O} \subset \mathbb{R}^{s+1}} \mathfrak{A}(\mathcal{O})$ into subspaces which differ in their behavior under translations in space. We first identify the pure-point and the continuous subspace in Subsection 2.2.1. Next, in Subsection 2.2.2, we decompose the continuous subspace into suitably defined absolutely continuous and point-continuous parts. The latter subspace is a new feature which does not have a counterpart in the Hilbert space setting considered in the previous section. Since we abstracted our decomposition from the study of physically relevant examples, discussed in Subsection 2.2.3 we do not expect any counterpart of the singularcontinuous subspace to appear. In fact, in the quantum-mechanical framework outlined in Section 1.1 the singular-continuous subspace is trivial in models with complete particle interpretation.

\subsubsection{Pure-Point and Continuous Subspace}

In order to determine the pure-point spectrum, suppose that $A \in \hat{\mathfrak{A}}$ is an eigenvector of the translation automorphisms $\mathbb{R}^{s} \ni \vec{x} \rightarrow \alpha_{\vec{x}}$ i.e.

$$
\alpha_{\vec{x}}(A)=e^{-i \vec{q} \vec{x}} A, \quad \vec{x} \in \mathbb{R}^{s}
$$

for some $\vec{q} \in \mathbb{R}^{s}$. Then $A$ belongs to the center of $\mathfrak{A}$, since locality gives

$$
\|[A, B]\|=\lim _{|\vec{x}| \rightarrow \infty}\left\|\left[\alpha_{\vec{x}}(A), B\right]\right\|=0, \quad B \in \mathfrak{A} .
$$

The irreducibility assumption from Section 1.6 ensures that the center of $\mathfrak{A}$ consists only of multiples of the unity. Hence the pure-point subspace is given by

$$
\hat{\mathfrak{A}}_{\mathrm{pp}}=\{\lambda I \mid \lambda \in \mathbb{C}\} .
$$


Since we do not have the concept of orthogonality, it is a priori not clear how to choose the complementing continuous subspace. In order to restrict the family of admissible projections $P_{\{0\}}$ on $\hat{\mathfrak{A}}_{\mathrm{pp}}$, we proceed along the lines set in the previous section: we introduce the following family of approximants

$$
P_{\{0\}, K}(A)=\frac{1}{|K|} \int_{K} d^{s} x \alpha_{\vec{x}}(A), \quad A \in \hat{\mathfrak{A}},
$$

which are defined as weak integrals for any compact subset $K \subset \mathbb{R}^{s}$. (They belong to $\hat{\mathfrak{A}}$ by the von Neumann bicommutant theorem). In the present setting we do not have the ergodic theorem at our disposal, hence it is not clear if the the above net converges as $K \nearrow \mathbb{R}^{s}$. However, there holds the following simple proposition. For reader's convenience we include the standard argument.

Proposition 2.2.1. There exists a net $\left\{K_{\beta} \subset \mathbb{R}^{s} \mid \beta \in \mathbb{I}\right\}$ of compact subsets of $\mathbb{R}^{s}$ s.t. $K_{\beta} \nearrow \mathbb{R}^{s}$ and for any $A \in \hat{\mathfrak{A}}$ there exists the limit in the weak $k^{*}$ topology of $B(\mathcal{H})$

$$
P_{\{0\}}(A):=\mathrm{w}^{*}-\lim _{\beta} \frac{1}{\left|K_{\beta}\right|} \int_{K_{\beta}} d^{s} x \alpha_{\vec{x}}(A) .
$$

Moreover, $P_{\{0\}}(A)=\omega_{0}(A) I$ for some state $\omega_{0} \in \mathfrak{A}^{*}$, which is invariant under spacetime translations and belongs to the closure of $\mathcal{T}_{E, 1}$, for any $E \geq 0$, in the weak $k^{*}$ topology of $\mathfrak{A}^{*}$.

Proof. For any normal functional $\varphi \in \mathcal{T}$ the function $\mathbb{R}^{s} \ni \vec{x} \rightarrow \alpha_{\vec{x}}^{*} \varphi$ is continuous w.r.t. the norm topology in $\mathcal{T}$. Given any compact subset $K \subset \mathbb{R}^{s}$, we define the functional $\varphi_{K} \in \mathcal{T}$ as the Bochner integral

$$
\varphi_{K}=\frac{1}{|K|} \int_{K} d^{s} x \alpha_{\vec{x}}^{*} \varphi
$$

Now we fix a state $\omega \in \mathcal{T}$ and obtain, from the Banach-Alaoglu theorem, a net $\left\{K_{\beta} \subset\right.$ $\left.\mathbb{R}^{s} \mid \beta \in \mathbb{I}\right\}$ and a state $\omega_{0} \in \mathfrak{A}^{*}$ s.t.

$$
\lim _{\beta} \omega_{K_{\beta}}(A)=\omega_{0}(A), \quad A \in \mathfrak{A} .
$$

By locality, $\left\{P_{\{0\}, K}(A)\right\}_{K \subset \mathbb{R}^{s}}$ is a central net, i.e. for any $B \in \mathfrak{A}$ there holds

$$
\lim _{K / \mathbb{R}^{s}}\left\|\left[P_{\{0\}, K}(A), B\right]\right\|=0 .
$$

Therefore, all its limit points w.r.t. the weak* topology of $B(\mathcal{H})$ are multiples of the identity, by the assumed irreducibility of $\mathfrak{A}$. It follows that for any $\varphi \in \mathcal{T}, A \in \mathfrak{A}$

$$
\lim _{K / \mathbb{R}^{s}}\left(\varphi_{K}(A)-\omega_{K}(A) \varphi(I)\right)=0
$$

Consequently

$$
\mathrm{w}^{*}-\lim _{\beta} \frac{1}{\left|K_{\beta}\right|} \int_{K_{\beta}} d^{s} x \alpha_{\vec{x}}(A)=\omega_{0}(A) I .
$$

Moreover, by choosing $\varphi=\alpha_{y}^{*} \omega$ for some $y \in \mathbb{R}^{s+1}$, we obtain from (2.2.9) that $\omega_{0}$ is translationally invariant. Similarly, noting that if $\varphi \in \mathcal{T}_{E, 1}$ then $\varphi_{K} \in \mathcal{T}_{E, 1}$, we obtain 
that $\omega_{0}$ belongs to the weak ${ }^{*}$ closure of $\mathcal{T}_{E, 1}$ in $\mathfrak{A}^{*}$ for any $E \geq 0$.

In contrast to the ergodic theorem from the Hilbert space setting, this proposition does not provide us with a unique projection on the pure-point subspace. However, it restricts the admissible projections to expressions of the form $P_{\{0\}}(\cdot)=\omega_{0}(\cdot) I$ where $\omega_{0}$ is a translationally invariant, energetically accessible 1 state. A thorough discussion of such states is the subject of Chapter [3, where the general postulates from Section [1.6 are supplemented with physically motivated phase space criteria. It is shown that under the existing Condition $C_{\sharp}$ BP90 every state $\omega_{0}$ satisfying the conditions from Proposition 2.2 .1 is a vacuum state, as defined in Section 1.6. More importantly, we introduce new phase space conditions $C_{\mathrm{b}}$ and $C_{\mathrm{\natural}}$, the former inspired by the behavior of coincidence measurements, the latter motivated by the physical principle of additivity of energy, and show that each of them entails the uniqueness of the energetically accessible vacuum state. These results provide evidence to the effect that in a large class of physically relevant models there holds the following condition, which we adopt as a standing assumption in this chapter.

Condition $V$ : A state $\omega_{0} \in \mathfrak{A}^{*}$, which is invariant under spacetime translations and can be approximated by elements from $\mathcal{T}_{E, 1}$ for some $E \geq 0$ in the weak* topology of $\mathfrak{A}^{*}$, is unique and is a vacuum state.

Under this condition the projection on the pure-point subspace $P_{\{0\}}(\cdot)=\omega_{0}(\cdot) I$ is fixed by the unique, energetically accessible vacuum state $\omega_{0}$. Thus we obtain from Proposition 2.2.1 the following ergodic theorem for translation automorphisms in QFT:

Theorem 2.2.2. Suppose that Condition $V$ holds. Then for any $A \in \hat{\mathfrak{A}}$ there exists the limit in the weak topology of $B(\mathcal{H})$

$$
P_{\{0\}}(A)=\mathrm{w}^{*}-\lim _{K \nearrow \mathbb{R}^{s}} \frac{1}{|K|} \int_{K} d^{s} x \alpha_{\vec{x}}(A) .
$$

Moreover, $P_{\{0\}}(A)=\omega_{0}(A) I$, where $\omega_{0} \in \mathfrak{A}^{*}$ is the unique energetically accessible vacuum state.

Guided by relation (2.1.3), we define the continuous subspace as $\hat{\mathfrak{A}}_{\mathrm{c}}=$ ker $P_{\{0\}}$ or equivalently

$$
\hat{\mathfrak{A}}_{\mathrm{c}}=\left\{A \in \hat{\mathfrak{A}} \mid \omega_{0}(A)=0\right\},
$$

where $\omega_{0}$ is the unique energetically accessible vacuum state appearing in Theorem 2.2.2. For future convenience we also define $\mathfrak{A}_{\mathrm{c}}(\mathcal{O})=\left\{A \in \mathfrak{A}(\mathcal{O}) \mid \omega_{0}(A)=0\right\}$.

\subsubsection{Absolutely Continuous and Point-Continuous Subspace}

Proceeding to more detailed analysis of the continuous subspace, we note that if Condition $V$ holds, then for any $A \in \hat{\mathfrak{A}}_{\mathrm{c}}$, spacelike unit vector $\hat{e} \in \mathbb{R}^{s+1}$, and $E \geq 0$, we get

$$
\lim _{\lambda \rightarrow \infty} \varphi\left(\alpha_{\lambda \hat{e}}(A)\right)=0, \quad \varphi \in \mathcal{T}_{E} .
$$

\footnotetext{
${ }^{1}$ We call a state $\omega \in \mathfrak{A}^{*}$ energetically accessible if it belongs to the closure of $\mathcal{T}_{E, 1}$ for some $E \geq 0$ in the weak ${ }^{*}$-topology of $\mathfrak{A}^{*}$.
} 
This is justified making use of the fact that $\left\{\alpha_{\lambda \hat{e}}(A)\right\}_{\lambda \geq 0}$ is a central net and proceeding as in the proof of Proposition 2.2.1. (See also Proposition 3.1.4 below). In view of this property, we can base further decomposition of $\hat{\mathfrak{A}}_{\mathrm{c}}$ on decay properties of the functions $\mathbb{R}^{s} \ni \vec{x} \rightarrow \varphi\left(\alpha_{\vec{x}} A\right)$. Guided by our discussion in Section [2.1] we expect that the best possible generic property of such functions should be square-integrability. Let us now demonstrate that in any quantum field theory there is a non-trivial subspace of local operators which are square-integrable in the sense made precise in Proposition 2.2.3 below. For this purpose we consider the operator-valued distribution

$$
\widetilde{A}(\vec{p})=\frac{1}{(2 \pi)^{\frac{s}{2}}} \int d^{s} p e^{i \vec{p} \vec{x}} \alpha_{\vec{x}}(A), \quad A \in \hat{\mathfrak{A}} .
$$

If $A \notin \hat{\mathfrak{A}}_{\mathrm{pp}}$, then the support of this distribution (i.e. the Arveson spectrum of $A$ w.r.t. space translations) coincides with $\mathbb{R}^{s}$ Bu90. A more detailed spectral theory should determine not only the support, but also regularity properties of the distribution (2.2.14). As we mentioned in Section [1.6. the first step in this direction was taken by Buchholz who has shown that in any local, relativistic QFT there holds the following bound

$$
\sup _{\varphi \in \mathcal{T}_{E, 1}} \int d^{s} p|\vec{p}|^{s+1+\varepsilon}|\varphi(\widetilde{A}(\vec{p}))|^{2}<\infty, \quad A \in \hat{\mathfrak{A}}
$$

for any $E \geq 0, \varepsilon>0$ Bu90. Making use of the fact that for any $f \in S\left(\mathbb{R}^{s}\right)$ there holds

$$
\widetilde{A(f)}(\vec{p})=(2 \pi)^{\frac{s}{2}} \tilde{f}(\vec{p}) \widetilde{A}(\vec{p}),
$$

where $A(f)=\int d^{s} x \alpha_{\vec{x}}(A) f(\vec{x})$, it is not difficult to find non-trivial operators $A \in \hat{\mathfrak{A}}_{\mathrm{c}}$ which satisfy a stronger estimate.

Proposition 2.2.3. In any quantum field theory admitting a vacuum state $\omega_{0} \in \mathfrak{A}^{*}$, there exist $0 \neq A \in \hat{\mathfrak{A}}$ s.t. $\omega_{0}(A)=0$ and

$$
\|A\|_{E, 2}:=\sup _{\varphi \in \mathcal{T}_{E, 1}}\left(\int d^{s} p|\varphi(\widetilde{A}(\vec{p}))|^{2}\right)^{\frac{1}{2}}<\infty .
$$

Proof. We pick $A \notin \hat{\mathfrak{A}}_{\mathrm{pp}}$. Since the support of the distribution $\mathbb{R}^{s} \ni \vec{p} \rightarrow \widetilde{A}(\vec{p})$ coincides with $\mathbb{R}^{s}$ and the space $C_{0}^{\infty}\left(\mathbb{R}^{s}\right)$ of smooth, compactly supported functions is dense in $S\left(\mathbb{R}^{s}\right)$ we can find a function $f \in C_{0}^{\infty}\left(\mathbb{R}^{s}\right)$ s.t. $A(f)=\widetilde{A}(\tilde{f}) \neq 0$. Next, for any $n \in \mathbb{N}$ we introduce functions $f_{n} \in C_{0}^{\infty}\left(\mathbb{R}^{s}\right)$ given by

$$
\tilde{f}_{n}(\vec{p})=\tilde{f}(\vec{p})|\vec{p}|^{2 n} .
$$

Then the operators $A\left(f_{n}\right) \in \hat{\mathfrak{A}}$ are also different from zero. (Otherwise the support of $\vec{p} \rightarrow \widetilde{A}(\vec{p})$ would have to be contained in $\{0\})$. Setting $4 n>s+1$ we obtain from identity (2.2.16) and estimate (2.2.15) that $\left\|A\left(f_{n}\right)\right\|_{E, 2}<\infty$ for any $E \geq 0$. In order to verify that $\omega_{0}\left(A\left(f_{n}\right)\right)=0$, we use local normality of the state $\omega_{0}$ to exchange its action with integration and the fact that $\tilde{f}_{n}(0)=0$.

We call operators $A \in \hat{\mathfrak{A}}_{\mathrm{c}}$, which satisfy the bound (2.2.17) for any $E \geq 0$, squareintegrable. We know from Section 2.1 that the quantum-mechanical analogue of this property implies the absolute continuity of the spectral measure and cannot be improved 
to integrability with any smaller power. Thus we define the absolutely continuous subspace of $\hat{\mathfrak{A}}_{\mathrm{c}}$ as consisting of all the square-integrable observables

$$
\hat{\mathfrak{A}}_{\mathrm{ac}}=\left\{A \in \hat{\mathfrak{A}}_{\mathrm{c}} \mid\|A\|_{E, 2}<\infty \text { for any } E \geq 0\right\} .
$$

This subspace is non-trivial in any local, relativistic QFT by Proposition 2.2.3 and we expect from Lemma 2.1.2 that it is large in a certain sense. Also our discussion of quantum mechanical scattering in Section 1.1 suggests that the absolutely continuous subspace should exhaust the continuous subspace in physically relevant models. In quantum field theory such situation prevails in massive models, admitting sufficiently many particle detectors, as we show in Theorem 2.3.1 below. However, in Subsection 2.2.3 we demonstrate that in a large family of massless theories the subspace $\hat{\mathfrak{A}}_{\text {ac }}$ has non-zero, but finite co-dimension in $\hat{\mathfrak{A}}_{\mathrm{c}}$. This case study reveals a class of models in which the absolutely continuous subspace has the following characterization:

Condition $L^{(2)}$ : There exists a finite family of (possibly unbounded) linear functionals $\tau_{1}, \ldots, \tau_{n}$ on $\hat{\mathfrak{A}}$, invariant under translations in space, s.t.

$$
\hat{\mathfrak{A}}_{\mathrm{ac}}=\operatorname{ker} \omega_{0} \cap \operatorname{ker} \tau_{1} \cap \ldots \cap \operatorname{ker} \tau_{n} .
$$

We find it noteworthy that the square-integrability requirement from definition (2.2.19) can, in many cases, be replaced with the linear-algebraic condition (2.2.20). The problem of constructing the distinguished family of functionals $\left\{\tau_{j}\right\}_{1}^{n}$ in particular examples is treated in Appendix C, Here we remark that in theories complying with Condition $L^{(2)}$ the direct sum complement of $\hat{\mathfrak{A}}_{\mathrm{ac}}$ in $\hat{\mathfrak{A}}_{\mathrm{c}}$ exists and is finite dimensional. This latter property makes it similar to the pure-point subspace, although it certainly belongs to $\hat{\mathfrak{A}}_{c}$. Therefore, we propose the term point-continuous subspace $\hat{\mathfrak{A}}_{\mathrm{pc}}$ for this direct sum complement. Thus we arrive at the decomposition

$$
\hat{\mathfrak{A}}=\hat{\mathfrak{A}}_{\mathrm{pp}} \oplus \hat{\mathfrak{A}}_{\mathrm{pc}} \oplus \hat{\mathfrak{A}}_{\mathrm{ac}} .
$$

Of course, the point-continuous subspace is non-unique and we do not introduce any natural choice here. However, its dimension and the behavior of its elements under translations, which we study below, do not depend on the selection which is made at this point.

If the point-continuous subspace is non-trivial, the continuous subspace contains observables which are not square-integrable. It is our next goal to quantify their deviation from the square-integrability. For this purpose we introduce the concept of the infrared order of an observable $A \in \hat{\mathfrak{A}}_{\mathrm{c}}$ :

$$
\operatorname{ord}(A):=\inf \left\{\beta \geq\left. 0\left|\sup _{\varphi \in \mathcal{T}_{E, 1}} \int d^{s} p\right| \vec{p}\right|^{\beta}|\varphi(\widetilde{A}(\vec{p}))|^{2}<\infty \text { for all } E \geq 0\right\} .
$$

Moreover, we define the set $\operatorname{Ord}\left(\hat{\mathfrak{A}}_{\mathrm{c}}\right)=\left\{\operatorname{ord}(A) \mid A \in \hat{\mathfrak{A}}_{\mathrm{c}}\right\}$. It follows from estimate (2.2.15), due to Buchholz, that ord $(A) \leq s+1$ for any $A \in \hat{\mathfrak{A}}_{\mathrm{c}}$ in any local, relativistic QFT. However, the models studied in Subsection 2.2 .3 below are far from saturating this bound. These examples demonstrate that the detailed regularity properties of the distributions (2.2.14), which are captured by the dimension of the point-continuous subspace and the infrared orders of its elements, can provide a distinction between different theories. In contrast, as we mentioned above, the Arveson spectrum of any $A \in \hat{\mathfrak{A}}_{\mathrm{c}}$ w.r.t. the space translations $\mathbb{R}^{s} \ni \vec{x} \rightarrow \alpha_{\vec{x}}$ coincides with $\mathbb{R}^{s}$. 


\subsubsection{Instructive Examples}

In this subsection we study briefly the spectral decomposition in scalar free field theory and related models, introduced in Section B.2. This discussion substantiates the point that the detailed spectral concepts, introduced in the previous subsection, provide a distinction between different models.

The first pair of examples are massive scalar free field theory and its even part, defined in Section B.2. As we expect perfectly regular infrared structure here, these models serve as a reference point for our later discussion. In fact there holds:

Theorem 2.2.4. Massive scalar free field theory and its even part satisfy Condition $L^{(2)}$ and have trivial point-continuous subspace for any dimension of space $s \geq 1$.

This result can be extracted from our discussion in Section 2.3 below: There we formulate Condition $L^{(1)}$ (a) which, roughly speaking, assures that the theory admits sufficiently many particle detectors. We verify in Appendix $\mathrm{D}$ that massive scalar free field theory and its even part satisfy this criterion. Then Theorem 2.3.1 entails the above result.

Let us now turn to the more interesting massless case. To exclude from consideration the unduly complicated infrared structure of low dimensional massless models, we set $s \geq 3$. Let $(\mathfrak{A}, \alpha, \mathcal{H})$ be the local net generated by the massless scalar free field acting on the Fock space $\mathcal{H}$. Let $\left(\underline{\mathfrak{A}}^{(\mathrm{e})}, \underline{\alpha}, \mathcal{H}^{(\mathrm{e})}\right)$ be the even part of this theory, that is the local net generated by even polynomials of the field acting on the even part $\mathcal{H}^{(\mathrm{e})}$ of the Fock space. Finally, we denote by $\left(\mathfrak{A}^{(\mathrm{d})}, \alpha, \mathcal{H}\right)$ the sub-theory of massless scalar free field theory generated by the derivatives of the massless scalar field. For precise definitions of these models see Section B.2. Condition $V$, which is the starting point of our spectral analysis, is verified in these examples in Theorem 3.4 .3 and Appendix E For the proofs of the three theorems stated below consult Appendix [C]

The following theorem describes the dimensions of the point-continuous subspace and the infrared orders of its elements in the full massless scalar free field theory.

Theorem 2.2.5. Massless scalar free field theory satisfies Condition $L^{(2)}$ for $s \geq 3$. Moreover, there hold the following statements:

(a) If $s=3$, then $\operatorname{dim} \hat{\mathfrak{A}}_{\mathrm{pc}}=2$ or 3 and $\operatorname{Ord}\left(\hat{\mathfrak{A}}_{\mathrm{c}}\right)=\{0,1,2\}$.

(b) If $s=4$, then $\operatorname{dim} \hat{\mathfrak{A}}_{\mathrm{pc}}=1$ or 2 and $\operatorname{Ord}\left(\hat{\mathfrak{A}}_{\mathrm{c}}\right)=\{0,2\}$.

(c) If $s \geq 5$, then $\operatorname{dim} \hat{\mathfrak{A}}_{\mathrm{pc}}=1$ and $\operatorname{Ord}\left(\hat{\mathfrak{A}}_{\mathrm{c}}\right)=\{0,2\}$.

Clearly, the situation differs substantially from the massive case mentioned above. As expected, the infrared structure improves with increasing dimension, in the sense that the dimension of the point-continuous subspace decreases. However, this subspace remains non-trivial for any $s \geq 3$. In parts (a) and (b) of the above theorem further investigation is needed to determine the dimension of $\hat{\mathfrak{A}}_{\mathrm{pc}}$ exactly.

The next example which we present is the even part of massless free field theory. The following result demonstrates that the infrared structure is significantly modified if one restricts attention to even observables.

Theorem 2.2.6. The even part $\left(\underline{\mathfrak{A}}^{(e)}, \underline{\alpha}, \mathcal{H}^{(e)}\right)$ of massless scalar free field theory satisfies Condition $L^{(2)}$ for $s \geq 3$ and there hold the following statements: 
(a) If $s=3$, then $\operatorname{dim} \underline{\hat{\mathfrak{A}}}_{\mathrm{pc}}^{(e)}=1$ and $\operatorname{Ord}\left(\underline{\hat{\mathfrak{A}}}_{\mathrm{c}}^{(e)}\right)=\{0,1\}$.

(b) If $s=4$, then $\operatorname{dim} \underline{\hat{\mathfrak{A}}}_{\mathrm{pc}}^{(e)}=0$ or 1 and $\operatorname{Ord}\left(\underline{\hat{\mathfrak{A}}}_{\mathrm{c}}^{(e)}\right)=\{0\}$.

(c) If $s \geq 5$, then $\operatorname{dim} \underline{\hat{\mathfrak{A}}}_{\mathrm{pc}}^{(e)}=0$.

Here the point-continuous subspace is again non-trivial in physical spacetime, but disappears in higher dimensions. We note that the maximal infrared order of local observables in this model is strictly smaller than the corresponding quantity in the full theory for any dimension of space $s \geq 3$. Again, the precise dimension of the point-continuous subspace in part (b) remains to be determined.

Our last example is the sub-theory of massless free field theory generated by the derivatives of the field.

Theorem 2.2.7. The sub-theory $\left(\mathfrak{A}^{(d)}, \alpha, \mathcal{H}\right)$ of massless scalar free field theory satisfies Condition $L^{(2)}$ and has trivial point-continuous subspace for any dimension of space $s \geq 3$.

This theorem demonstrates that triviality of the point-continuous subspace is not a characteristic feature of massive models. Such mild infrared structure may occur also in massless theories.

The case study, presented in this subsection, demonstrates that the detailed spectral concepts of the point-continuous subspace and the infrared order provide quantitative means to compare the infrared behavior of different quantum field theory models. For example, for $s=3$ there holds

$$
\operatorname{dim} \hat{\mathfrak{A}}_{\mathrm{pc}}>\operatorname{dim} \underline{\hat{\mathfrak{A}}}_{\mathrm{pc}}^{(\mathrm{e})}>\operatorname{dim} \hat{\mathfrak{A}}_{\mathrm{pc}}^{(\mathrm{d})},
$$

thus we can meaningfully order the three massless models under study w.r.t. their infrared properties. Presumably, more detailed spectral analysis is needed to distinguish between massive scalar free field theory and $\left(\mathfrak{A}^{(\mathrm{d})}, \alpha, \mathcal{H}\right)$. We expect that allowing for negative values of the infrared order (see formula (2.2.22) ) could serve this purpose, but computations remain to be done.

\subsection{Triviality of Point-Continuous Subspace and Existence of Particles}

In the previous section we constructed a general decomposition of the algebra of observables $\hat{\mathfrak{A}}$ into subspaces which differ in their behavior under translations in space. Moreover, we identified the point-continuous subspace $\hat{\mathfrak{A}}_{\mathrm{pc}}$ which carries information about the infrared structure. In this section we formulate a condition which characterizes a class of theories with $\hat{\mathfrak{A}}_{\mathrm{pc}}=\{0\}$. We show that models complying with this assumption and admitting in addition a stress-energy tensor, have non-trivial particle content.

We argued in Section 2.1] that the square-integrability under translations in space should be the best possible generic feature of local operators. However, almost local operators may have much better integrability properties. In fact, Theorem 1.6.1] due to Buchholz gives us a large class of observables $C \in \mathfrak{A}$ which satisfy

$$
\|C\|_{E, 1}:=\sup _{\varphi \in \mathcal{T}_{E, 1}} \int d^{s} x|\varphi(C(\vec{x}))|<\infty .
$$


The observables $C \in \mathfrak{A}$ which satisfy the above bound for any $E \geq 0$ will be called integrable. A natural framework for our investigations is based on the subspace of $\mathfrak{A}$ spanned by all such integrable operators:

$$
\mathfrak{A}^{(1)}=\left\{C \in \mathfrak{A} \mid\|C\|_{E, 1}<\infty \text { for any } E \geq 0\right\} .
$$

Equipped with the family of seminorms $\left\{\|\cdot\|_{E, 1} \mid E \geq 0\right\}$, the space $\mathfrak{A}^{(1)}$ is a locally convex Hausdorff space and we call the corresponding topology $T^{(1)}$. (This is established as in Section 2.2 of [P04.1]). The new Condition $L^{(1)}$, stated below, specifies another class of integrable, almost local observables which, as we shall see below, can be used to approximate the stress-energy tensor. These observables are of the form $A(g)=\int d t \alpha_{t}(A) g(t)$, where $A \in \hat{\mathfrak{A}}_{\mathrm{c}}$ and $g \in S(\mathbb{R})$ is a suitable time-smearing function.

Condition $L^{(1)}$ : There exists such $\mu>0$ that for any $g \in S(\mathbb{R})$ s.t. $\left.\operatorname{supp} \widetilde{g} \subset\right]-\mu, \mu[$ there hold the assertions:

(a) $A(g) \in \mathfrak{A}^{(1)}$ for any $A \in \hat{\mathfrak{A}}_{\mathrm{c}}$,

(b) $\|A(g)\|_{E, 1} \leq c_{l}\left\|R^{l} A R^{l}\right\|$ for any $A \in \mathfrak{A}_{\mathrm{c}}(\mathcal{O}), l \geq 0$,

where $R=(1+H)^{-1}$ and the constant $c_{l}$ depends also on $\mathcal{O}, E \geq 0$ and $g$.

This condition seems to be a generic property of purely massive theories. We verify it in massive scalar free field theory in Appendix D Part (a) is verified also in the even part of this theory (defined in Section B.2) making use of the fact that the norm of an (even) local operator $A$ does not change upon its restriction to the even subspace of the Fock space (cf. formula (B.2.17) ). This property reflects the fact that a state consisting of an odd number of particles can be approximated in any bounded region of spacetime by a state consisting of an even number of particles by shifting one particle to spacelike infinity. We expect that a similar reasoning can be applied to the dislocalized operators $R^{l} A R^{l}$, appearing in part (b) of the condition, but the computation remains to be done. We also conjecture that the above criterion can be established in theories of charged, non-interacting massive particles.

Now we are ready to prove the main spectral result of this section, namely that theories satisfying Condition $L^{(1)}$ (a) have trivial point-continuous subspace. In other words, we show that Condition $L^{(1)}$ (a) implies Condition $L^{(2)}$ with the functionals $\left\{\tau_{i}\right\}_{1}^{n}$ equal to zero.

Theorem 2.3.1. Assume that Condition $L^{(1)}$ (a) holds. Then $\hat{\mathfrak{A}}_{\mathrm{pc}}=\{0\}$.

Proof. It suffices to show that for any $A \in \hat{\mathfrak{A}}_{\mathrm{c}}$ and any $E>\mu$, where $\mu$ appeared in Condition $L^{(1)}$ (a), there holds $\|A\|_{E, 2}<\infty$. To this end, we choose a function $f \in S(\mathbb{R})$ s.t. $\tilde{f}=(2 \pi)^{-\frac{1}{2}}$ on $[-E, E]$ and $\operatorname{supp} \tilde{f} \subset[-2 E, 2 E]$. With the help of a smooth partition of unity we can decompose $f$ as follows: $f=f_{-}+f_{+}+f_{0}$, where supp $\tilde{f}_{-} \subset[-2 E,-\mu / 2]$, $\operatorname{supp} \tilde{f}_{+} \subset[\mu / 2,2 E]$, and $\left.\operatorname{supp} \tilde{f}_{0} \subset\right]-\mu, \mu[$. Then there holds

$$
P_{E} A P_{E}=P_{E} A(f) P_{E}=P_{E} A\left(f_{-}\right) P_{E}+P_{E} A\left(f_{+}\right) P_{E}+P_{E} A\left(f_{0}\right) P_{E} .
$$

We note that $A\left(f_{0}\right)$ is square-integrable (and even integrable) by Condition $L^{(1)}$ (a). To the remaining terms we can apply Theorem 1.6.1 since both $A\left(f_{-}\right)$and $A\left(f_{+}\right)^{*}$ are almost local and energy-decreasing. This latter statement follows from the equality

$$
\widetilde{A\left(f_{-}\right)}(p)=(2 \pi)^{\frac{1}{2}} \tilde{f}_{-}\left(p^{0}\right) \widetilde{A}\left(p^{0}, \vec{p}\right)
$$


which implies that the support of $\widetilde{A\left(f_{-}\right)}$does not intersect with the closed future lightcone. (An analogous argument applies to $\left.A\left(f_{+}\right)^{*}\right)$. Thus we obtain from Theorem 1.6.1. for any compact subset $K \subset \mathbb{R}^{s}$,

$$
\sup _{\omega \in S_{E}} \int_{K} d^{s} x\left|\omega\left(A\left(f_{-}\right)(\vec{x})\right)\right|^{2} \leq \sup _{\omega \in S_{E}} \int_{K} d^{s} x \omega\left(\left(A\left(f_{-}\right)^{*} A\left(f_{-}\right)\right)(\vec{x})\right) \leq c_{E} .
$$

Here $c_{E}$ is independent of $K$ hence we can take the limit $K \nearrow \mathbb{R}^{s}$ and the claim follows from decomposition (1.6.2) and definition (2.2.17) of the seminorms $\|\cdot\|_{E, 2}$. The case of $A\left(f_{+}\right)$is treated analogously.

Proceeding to the particle aspects of the theory, we define, for any $\varphi \in \mathcal{T}_{E}$, the net $\left\{\sigma_{\varphi}^{(t)}\right\}_{t \in \mathbb{R}_{+}}$of functionals on $\mathfrak{A}^{(1)}$ given by

$$
\sigma_{\varphi}^{(t)}(C)=\int d^{s} x \varphi\left(\alpha_{t, \vec{x}}(C)\right)
$$

This net satisfies the uniform bound $\left|\sigma_{\varphi}^{(t)}(C)\right| \leq\|\varphi\|\|C\|_{E, 1}$. Therefore, by the AlaogluBourbaki theorem (see [Ja], Section 8.5), it has limit points $\sigma_{\omega}^{(+)}$in the topological dual of $\left(\mathfrak{A}^{(1)}, T^{(1)}\right)$ which are called the asymptotic functionals. The set of such functionals

$$
\mathfrak{P}=\left\{\sigma_{\varphi}^{(+)} \mid \varphi \in \mathcal{T}_{E} \text { for some } E \geq 0\right\}
$$

is called the particle content of a theory. This terminology is justified by the fact that in asymptotically complete theories of Wigner particles, discussed in Section 1.2 these functionals are related to the asymptotic particle density. In fact, for one species of Wigner particles, and $C=B^{*} B$, where $B$ is almost local and energy-decreasing, there holds AH67, En75.

$$
\sigma_{\varphi}^{(+)}(C)=(2 \pi)^{s} \int d^{s} p \varphi\left(a^{* \text { out }}(\vec{p}) a^{\text {out }}(\vec{p})\right)\langle\vec{p}|C| \vec{p}\rangle,
$$

where $a^{* \text { out }}(\vec{p}), a^{\text {out }}(\vec{p})$ are the creation and annihilation operators of the mode $\vec{p}$ from the Fock space of outgoing states and the kernels $\vec{p} \rightarrow\langle\vec{p}|C| \vec{p}\rangle$ are smooth, positive functions which are different from zero for suitably chosen $B$. This guarantees the existence of non-trivial asymptotic functionals $\sigma_{\varphi}^{(+)}$in this restrictive context. It is now our goal to show that $\mathfrak{P} \neq\{0\}$ not relying directly on the Wigner concept of a particle. Since our argument is based on the existence of the stress-energy tensor, which is postulated in Condition $T$ below, we recall the definition and properties of pointlike-localized fields: We set $R=(1+H)^{-1}$ and introduce the space of normal functionals with polynomially damped energy

$$
\mathcal{T}_{\infty}=\bigcap_{l \geq 0} R^{l} \mathcal{T} R^{l}
$$

where $\mathcal{T}=B(\mathcal{H})_{*}$. We equip this space with the locally convex topology given by the norms $\|\cdot\|_{l}=\left\|R^{-l} \cdot R^{-l}\right\|$ for $l \geq 0$. The field content of a theory is defined as follows FH81

$$
\Phi_{\mathrm{FH}}=\left\{\phi \in \mathcal{T}_{\infty}^{*} \mid R^{l} \phi R^{l} \in \bigcap_{r>0}{\overline{R^{l} \mathfrak{A}(\mathcal{O}(r)) R^{l}}}^{w} \text { for some } l \geq 0\right\}
$$


There holds the following useful approximation property for pointlike-localized fields which is due to Bostelmann [Bos05.1]: For any $\phi \in \Phi_{\mathrm{FH}}$ there exists $l \geq 0$ and a net $A_{r} \in$ $\mathfrak{A}(\mathcal{O}(r)), r>0$, s.t.

$$
\lim _{r \rightarrow 0}\left\|R^{l}\left(A_{r}-\phi\right) R^{l}\right\|=0 .
$$

It follows from Proposition 2.2 .1 that for any $E \geq 0$ one can choose a net $\left\{\varphi_{\beta}\right\}_{\beta \in \mathbb{I}}$ of functionals from $\mathcal{T}_{E, 1}$, approximating $\omega_{0}$ in the weak* topology of $\mathfrak{A}^{*}$. Therefore, we obtain

$$
\left|\omega_{0}(A)\right| \leq\left\|P_{E} A P_{E}\right\|, \quad A \in \mathfrak{A},
$$

and the approximation property (2.3.11) implies that $\omega_{0}$ can be uniquely extended to elements from $\Phi_{\mathrm{FH}}$. We can therefore introduce the continuous part of this space

$$
\Phi_{\mathrm{FH}, \mathrm{c}}=\left\{\phi \in \Phi_{\mathrm{FH}} \mid \omega_{0}(\phi)=0\right\} .
$$

The approximation property (2.3.11) can now be restated as follows: For any $\phi \in \Phi_{\mathrm{FH}, \mathrm{c}}$ there exists $l \geq 0$ and $A_{r} \in \mathfrak{A}_{\mathrm{c}}(\mathcal{O}(r))$ s.t.

$$
\lim _{r \rightarrow 0}\left\|R^{l}\left(A_{r}-\phi\right) R^{l}\right\|=0 .
$$

Making use of Condition $L^{(1)}$ (b) we also obtain, for any time-smearing function $g \in S(\mathbb{R})$ s.t. $\operatorname{supp} \widetilde{g} \subset]-\mu, \mu[$,

$$
\lim _{r \rightarrow 0}\left\|A_{r}(g)-\phi(g)\right\|_{E, 1}=0 .
$$

This implies, in particular, that $\|\phi(g)\|_{E, 1}<\infty$ for any $\phi \in \Phi_{\mathrm{FH}, \mathrm{c}}$, what prepares the ground for our next assumption:

Condition $T$ : There exists a field $T^{00} \in \Phi_{\mathrm{FH}, \mathrm{c}}$ which satisfies

$$
\int d^{s} x \varphi\left(T^{00}(g)(\vec{x})\right)=\varphi(H), \quad \varphi \in \mathcal{T}_{E}
$$

for any $E \geq 0$ and time-smearing function $g \in S(\mathbb{R})$ s.t. $\operatorname{supp} \widetilde{g} \subset]-\mu, \mu[, \widetilde{g}(0)=$ $(2 \pi)^{-\frac{1}{2}}$.

This condition holds, in particular, in massive scalar free field theory and its even part as we show in Theorem B.2.1. With the stress-energy tensor at hand, it is easy to prove that the particle content of the theory is non-trivial:

Theorem 2.3.2. Suppose that a theory satisfies Conditions $L^{(1)}$ and $T$. Then, for any $\varphi \in \mathcal{T}_{E}$ s.t. $\varphi(H) \neq 0$, the corresponding asymptotic functionals satisfy $\sigma_{\varphi}^{(+)} \neq 0$.

Proof. We choose $g \in S(\mathbb{R})$ as in Condition $T$ and $0<\varepsilon \leq \frac{1}{2}|\varphi(H)|$. Making use of Condition $L^{(1)}$ (b) and relation (2.3.15), we can find $C \in \mathfrak{A}^{(1)}$ s.t. $\left\|T^{00}(g)-C\right\|_{E, 1} \leq \varepsilon$. Then, exploiting Condition $T$ and the invariance of $H$ under time translations, we obtain

$$
|\varphi(H)|=\left|\int d^{s} x \varphi\left(T^{00}(g)(\vec{x})\right)\right| \leq \varepsilon+\left|\int d^{s} x \varphi(C(t, \vec{x}))\right| .
$$

Thus we obtain a strictly positive lower bound on the asymptotic functional approximants, defined by formula (2.3.6), which is uniform in time. 
We emphasize that we have proven here more than non-triviality of the particle content - we have verified that every physical state, which has non-zero average energy, gives rise to a non-trivial asymptotic functional.

The framework, which we considered above, is more general than the one considered in the theory of particle weights developed by Buchholz, Porrmann and Stein [BPS91]. These authors introduced the left ideal

$$
\mathfrak{L}=\{A B \mid A, B \in \mathfrak{A}, B \text { is almost local and energy-decreasing }\}
$$

and proposed the $*$-algebra of particle detectors $\mathfrak{C}=\mathfrak{L}^{*} \mathfrak{L}$ as the domain of asymptotic functionals. It follows from Theorem 1.6.1, polarization identity and the bound $B^{*} A B \leq$ $\|A\| B^{*} B$, valid for any self-adjoint operator $A$, that $\mathfrak{C} \subset \mathfrak{A}^{(1)}$. Similarly, $\mathfrak{C}(g) \subset \mathfrak{A}^{(1)}$, where $\mathfrak{C}(g)=\{C(g) \mid C \in \mathfrak{C}\}$ for some time-smearing function $g \in S(\mathbb{R})$. As $\mathfrak{C}$ may be a proper subspace in $\mathfrak{A}^{(1)}$, it is not clear if the asymptotic functionals, which are non-trivial on $\mathfrak{A}^{(1)}$ by Theorem 2.3.2 remain non-trivial after restriction to $\mathfrak{C}$. This gap can be closed with the help of the following strengthened form of Condition $L^{(1)}$.

Condition $L_{\sharp}^{(1)}$ : There exists such $\mu>0$ that for any $g \in S(\mathbb{R})$ s.t. $\left.\operatorname{supp} \widetilde{g} \subset\right]-\mu, \mu[$ there hold the assertions:

(a) $A(g) \in \overline{\mathfrak{C}(g)}^{T(1)}$ for any $A \in \hat{\mathfrak{A}}_{\mathrm{c}}$,

(b) $\|A(g)\|_{E, 1} \leq c_{l}\left\|R^{l} A R^{l}\right\|$ for any $A \in \mathfrak{A}_{\mathrm{c}}(\mathcal{O}), l \geq 0$,

where the constant $c_{l}$ depends also on $\mathcal{O}, E \geq 0$ and $g$.

Making use of the fact that $\mathbb{R}_{+} \ni E \rightarrow\|C\|_{E, 1}$ is an increasing function for any fixed $C \in \mathfrak{A}^{(1)}$, part (a) of the above criterion can be restated as the requirement that for any $E \geq 0$ and $\varepsilon>0$ there exists $C \in \mathfrak{C}$ s.t.

$$
\|A(g)-C(g)\|_{E, 1} \leq \varepsilon
$$

We verify in Appendix $\mathrm{D}$ that this condition holds in massive scalar free field theory, hence it is consistent with the basic postulates. However, it seems to be too specific to hold in the even part of this theory (defined in Section B.2) or in models containing charged particles. As we mentioned above, in the restrictive framework of theories satisfying this condition, we can solve the problem of existence of non-trivial asymptotic functionals on $\mathfrak{C}$.

Theorem 2.3.3. Suppose that a theory satisfies Conditions $L_{\sharp}^{(1)}$ and $T$. Then, for any $\varphi \in \mathcal{T}_{E}$ s.t. $\varphi(H) \neq 0$, the corresponding asymptotic functional satisfies $\left.\sigma_{\varphi}^{(+)}\right|_{\mathfrak{C}} \neq 0$.

Proof. We choose $g \in S(\mathbb{R})$ as in Condition $T$. With the help of Condition $L_{\sharp}^{(1)}$ and formula (2.3.15) we can find, for any $\varepsilon>0$, a detector $C \in \mathfrak{C}$ s.t. $\left\|T^{00}(g)-C(g)\right\|_{E, 1} \leq \varepsilon$. There holds

$$
\begin{aligned}
|\varphi(H)| & =\left|\int d^{s} x \omega\left(T^{00}(g)(\vec{x})\right)\right| \leq \varepsilon+\left|\int d^{s} x \varphi(C(g)(t, \vec{x}))\right| \\
& =\varepsilon+\left|\int d \tau g(\tau) \int d^{s} x \omega(C(t+\tau, \vec{x}))\right|,
\end{aligned}
$$

where in the last step we made use of the fact that $\|C\|_{E, 1}<\infty$ and of the Fubini theorem to change the order of integration. Assuming that $\lim _{t \rightarrow \infty} \int d^{s} x \varphi(C(t, \vec{x}))=0$ and 
making use of the Lebesgue dominated convergence theorem, we arrive at a contradiction if $\varepsilon<|\varphi(H)|$.

We recall, that in the framework of Buchholz, Porrmann and Stein any asymptotic functional $\left.\sigma_{\omega}^{(+)}\right|_{\mathfrak{C}}$, where $\omega \in S_{E}$, can be decomposed into pure functionals $\sigma_{\lambda}^{(+)}$on $\mathfrak{C}$, the so called pure particle weight: 2

$$
\left.\sigma_{\omega}^{(+)}\right|_{\mathfrak{C}}=\int d \mu(\lambda) \sigma_{\lambda}^{(+)}
$$

which are labeled by sharp four-momentum $p_{\lambda}$ and a label $\gamma_{\lambda}$ which carries information about the internal degrees of freedom. This decomposition generalizes relation (2.3.8), valid in the framework of asymptotically complete Wigner particles. A possible formulation of the problem of asymptotic completeness in the general context was proposed by Buchholz in Bu94. In particular, it should be possible to determine the energy and momentum of any physical state $\omega \in S_{E}$, knowing its particle content from formula (2.3.21). That is, there should hold

$$
\omega(P)=\int d \mu(\lambda) p_{\lambda}
$$

where $P=(H, \vec{P})$ and the measure $d \mu$ is suitably normalized. It was conjectured in Bu94 that this relation holds in models admitting a stress-energy tensor. We note that Condition $L_{\sharp}^{(1)}$ provides a link between pointlike-localized fields and the algebra of particle detectors $\mathfrak{C}$ on which the decomposition (2.3.21) has been established (up to the technical details mentioned in the footnote). Therefore, our criterion should be useful for establishing this form of asymptotic completeness.

\footnotetext{
${ }^{2}$ As a matter of fact, the decomposition has been established only on some subalgebra of regular particle detectors in $\mathfrak{C}$. We refer to Po04.1 for details.
} 


\section{Chapter 3}

\section{Uniqueness of Spectral Decomposition and Vacuum Structure}

The decomposition of the algebra of local observables $\hat{\mathfrak{A}}$ into spectral subspaces, performed in the previous chapter, was based on Condition $V$, stated in Section 2.2. This criterion guarantees that a state which is translationally invariant and energetically accessible is unique and is a vacuum state. Among its consequences is the ergodic theorem 2.2.2 which gives the unique projection $P_{\{0\}}$ on the continuous subspace of the algebra of observables. It is the goal of the present section to derive Condition $V$ and other properties of vacuum states from physically motivated phase space conditions.

Physical properties of vacuum states were a subject of study since the early days of algebraic quantum field theory [BHS63, Bor65]. In particular, the problem of convergence of physical states to the vacuum state under large translations attracted much attention. It was considered under the assumptions of complete particle interpretation in the sense of Wigner AH67, isolated mass hyperboloid BF82 and asymptotic abelianess in time BWa92. As none of these assumptions is expected to hold in all physically relevant models, further investigation of the vacuum structure is warranted.

This subject is revisited here from the point of view of phase space analysis: We show in Section 3.1 that the well known compactness condition $C_{\sharp}$ implies that any physical state which is invariant under translations in some spacelike ray is a vacuum state. This applies, in particular, to the state $\omega_{0}$ which appears as a limit point of space averages in Proposition 2.2.1. In order to prove the uniqueness of the energetically accessible vacuum states, which entails the convergence of the space averages, we first reformulate Condition $C_{\sharp}$ in Section 3.2 so that it describes the behavior of coincidence arrangements of local operators. Next, in Section [3.3 we accompany it with a quantitative refinement, motivated by the fact that physical states are localized in space. We show that the resulting Condition $C_{\mathrm{b}}$ entails the purity and the uniqueness of vacuum states which can be prepared with a finite amount of energy. There follows Condition $V$ and, as a consequence, the ergodic theorem 2.2.2 In addition, we demonstrate that in Lorentz covariant theories there holds the convergence of physical states to the vacuum state under large timelike translations i.e. relaxation to the vacuum given by formula (1.3.1).

Since Condition $C_{\mathrm{b}}$ has clear physical interpretation and holds in massive scalar free 
field theory, as shown in Appendix E we expect that it is a generic feature of massive theories. However, its status in the realm of massless theories is not clear. Therefore, in Section 3.4 we formulate an alternative phase space condition $C_{\text {七 }}$, inspired by the physical principle of additivity of energy over isolated subregions, and show that it has all the physical consequences mentioned above (apart from the purity of the vacuum). It is verified in Appendix $\mathbb{E}$ that this criterion holds both in massive and massless theory of scalar non-interacting particles. In order to facilitate this argument, we introduce in Section 3.5 an auxiliary nuclearity condition $N_{\sharp}$, interesting in its own right, which entails Condition $C_{\text {七 }}$.

Some results from Sections 3.1 and 3.5 have been published by the author in Dy08.1.

\subsection{Condition $C_{\sharp}$ and Existence of Vacuum States}

It is well known from quantum mechanics that a system of bounded energy, restricted to a finite volume, should have a finite number of degrees of freedom. Following the seminal work of Haag and Swieca [HS65, this fact has been formulated in quantum field theory as compactness and nuclearity requirements on certain linear maps BWi86, BP90.

For any $E \geq 0, \beta>0$ and double cone $\mathcal{O}$ we consider the maps $\Pi_{E}: \mathcal{T}_{E} \rightarrow \mathfrak{A}(\mathcal{O})^{*}$, $\Theta_{E}: \mathfrak{A}(\mathcal{O}) \rightarrow B(\mathcal{H})$ and $\Xi_{\beta}: \mathfrak{A}(\mathcal{O}) \rightarrow B(\mathcal{H})$, given by

$$
\begin{aligned}
\Pi_{E}(\varphi) & =\left.\varphi\right|_{\mathfrak{A}(\mathcal{O})}, & & \varphi \in \mathcal{T}_{E}, \\
\Theta_{E}(A) & =P_{E} A P_{E}, & & A \in \mathfrak{A}(\mathcal{O}), \\
\Xi_{\beta}(A) & =e^{-\beta H} A e^{-\beta H}, & & A \in \mathfrak{A}(\mathcal{O}) .
\end{aligned}
$$

It is convenient to adopt here the restrictive definition of compactness from [BP90]: Let $V$ and $W$ be Banach spaces and let $\mathcal{L}(V, W)$ denote the space of linear maps from $V$ to $W$ equipped with the standard norm. Let $\mathcal{F}(V, W)$ be the subspace of finite rank mappings. More precisely, any $F \in \mathcal{F}(V, W)$ is of the form $F=\sum_{i=1}^{n} \tau_{i} S_{i}$, where $\tau_{i} \in W$ and $S_{i} \in V^{*}$. We say that a map $\Pi \in \mathcal{L}(V, W)$ is compact if it belongs to the closure of $\mathcal{F}(V, W)$ in the norm topology of $\mathcal{L}(V, W)$.

It was argued by Fredenhagen and Hertel in some unpublished work, quoted in [BP90], that in physically meaningful theories there should hold the following condition:

Condition $C_{\sharp}$ : The maps $\Pi_{E}$ are compact for any $E \geq 0$ and any double cone $\mathcal{O}$.

We readily obtain equivalent formulations of this criterion which are also useful. (A similar argument appears in [BP90]).

Lemma 3.1.1. We fix a double cone $\mathcal{O}$. Then the following conditions are equivalent:

(a) The maps $\Pi_{E}$ are compact for any $E \geq 0$.

(b) The maps $\Theta_{E}$ are compact for any $E \geq 0$.

(c) The maps $\Xi_{\beta}$ are compact for any $\beta>0$.

Proof. The implication $(a) \Rightarrow(b)$ can be shown as follows: Let $\varepsilon>0$ and $F \in$ $\mathcal{F}\left(\mathcal{T}_{E}, \mathfrak{A}(\mathcal{O})^{*}\right)$ be a finite rank map s.t. $\left\|\Pi_{E}-F\right\| \leq \varepsilon$. It has the form

$$
F=\sum_{i=1}^{n} \tau_{i} S_{i}
$$


for some $\tau_{i} \in \mathfrak{A}(\mathcal{O})^{*}$ and $S_{i} \in \mathcal{T}_{E}^{*}$. Every element $S_{i}$ can be extended, by the Hahn-Banach theorem, to an element $\widehat{S}_{i} \in B(\mathcal{H})$. Then the formula $\widehat{F}=\sum_{i=1}^{n}\left(P_{E} \widehat{S}_{i} P_{E}\right) \tau_{i}$ defines a map in $\mathcal{F}(\mathfrak{A}(\mathcal{O}), B(\mathcal{H}))$ which satisfies $\left\|\Theta_{E}-\widehat{F}\right\| \leq \varepsilon$. This is easily verified making use of the fact that for any $\varphi \in \mathcal{T}_{E}$ and $A \in \mathfrak{A}(\mathcal{O})$ there holds $\Pi_{E}(\varphi)(A)=\varphi\left(\Theta_{E}(A)\right)$. The implication $(b) \Rightarrow(a)$ can be shown by an analogous reasoning.

To verify $(b) \Rightarrow(c)$, we note that the spectral theorem gives

$$
\left\|\Xi_{\beta}(A)-e^{-\beta H} \Theta_{E}(A) e^{-\beta H}\right\| \leq 2\|A\| e^{-\beta E} .
$$

Thus, choosing sufficiently large $E$, we can approximate the map $\Xi_{\beta}$ by finite rank mappings up to arbitrary accuracy. The opposite implication follows from the identity $\Theta_{E}(A)=P_{E} e^{\beta H} \Xi_{\beta}(A) e^{\beta H} P_{E}$.

Turning to the vacuum structure, we note the following elementary lemma which ensures the local normality of the energetically accessible states in theories complying with Condition $C_{\sharp}$. (A similar argument appears in GJ70 p. 49).

Lemma 3.1.2. Suppose that Condition $C_{\sharp}$ holds. Let $\omega \in \mathfrak{A}^{*}$ be an element of the weak* closure of $\mathcal{T}_{E, 1}$ for some $E \geq 0$. Then $\omega$ is locally normal and can be approximated by a sequence of elements from $\mathcal{T}_{E, 1}$ in the weak $k^{*}$ topology.

Proof. First, we show that $\omega$ is locally normal. Let $\left\{\varphi_{\beta}\right\}_{\beta \in \mathbb{I}}$ be a net of elements of $\mathcal{T}_{E, 1}$ approximating $\omega$ in the weak* topology of $\mathfrak{A}^{*}$. By Condition $C_{\sharp}$, for any open bounded region $\mathcal{O}$ the set $\left\{\left.\varphi_{\beta}\right|_{\mathfrak{A}(\mathcal{O})} \mid \beta \in \mathbb{I}\right\}$ is compact in the norm topology of $\mathfrak{A}(\mathcal{O})^{*}$. We can therefore choose a subsequence $\left\{\varphi_{G(n)}\right\}_{n \in \mathbb{N}}$, where $G: \mathbb{N} \rightarrow \mathbb{I}$, approximating $\omega$ in this topology. Since a norm limit of a sequence of normal functionals is normal, we conclude that $\omega$ is a normal state upon restriction to any local algebra. Let $\mathcal{O}(m)$ denote the double cone of radius $m$. We note that by choosing subsequences $\left\{\varphi_{G_{m}(n)}\right\}_{n \in \mathbb{N}}$, converging in norm to $\omega$ on $\mathfrak{A}(\mathcal{O}(m))$, for any $m \in \mathbb{N}$, in such a way that $\left\{\varphi_{G_{m+1}(n)}\right\}_{n \in \mathbb{N}}$ is a subsequence of $\left\{\varphi_{G_{m}(n)}\right\}_{n \in \mathbb{N}}$, we obtain a diagonal sequence $\varphi_{n}=\varphi_{G_{n}(n)}$ which converges to $\omega$ in the weak* topology of $\mathfrak{A}^{*}$, replacing the original net.

We recall from Section [1.6 that a vacuum state is a state on $\mathfrak{A}$ which is translationally invariant, locally normal and s.t. the spectrum condition holds in its GNS-representation. In a theory satisfying Condition $C_{\sharp}$ there holds the following simple characterization of energetically accessible vacuum states.

Theorem 3.1.3. Suppose that Condition $C_{\sharp}$ holds. Let a state $\omega \in \mathfrak{A}^{*}$ be an element of the weak ${ }^{*}$ closure of $\mathcal{T}_{E, 1}$ for some $E \geq 0$ which is invariant under translations along some spacelike ray. Then $\omega$ is a vacuum state.

Proof. We pick any $A \in \mathfrak{A}(\mathcal{O})$, a test function $f \in S\left(\mathbb{R}^{s+1}\right)$ s.t. $\operatorname{supp} \tilde{f} \cap \bar{V}_{+}=\emptyset$ and define the energy-decreasing operator $A(f)=\int A(x) f(x) d^{s+1} x$. Next, we parametrize the ray from the statement of the theorem as $\{\lambda \hat{e} \mid \lambda \in \mathbb{R}\}$, where $\hat{e} \in \mathbb{R}^{s+1}$ is some spacelike unit vector, choose a compact subset $K \subset \mathbb{R}$ and estimate

$$
\begin{aligned}
\omega\left(A(f)^{*} A(f)\right)|K| & =\int_{K} d \lambda \omega\left(\left(A(f)^{*} A(f)\right)(\lambda \hat{e})\right) \\
& =\lim _{n \rightarrow \infty} \varphi_{n}\left(\int_{K} d \lambda\left(A(f)^{*} A(f)\right)(\lambda \hat{e})\right) \\
& \leq\left\|P_{E} \int_{K} d \lambda\left(A(f)^{*} A(f)\right)(\lambda \hat{e}) P_{E}\right\| .
\end{aligned}
$$


In the first step we exploited the invariance of the state $\omega$ under translations along the spacelike ray. In the second step we made use of the local normality of this state, (or of the dominated convergence theorem), in order to exchange its action with integration. Approximating $\omega$ by a sequence of functionals $\varphi_{n} \in \mathcal{T}_{E, 1}$, we arrived at the last expression. (The local normality of $\omega$ and the existence of an approximating sequence follow from Lemma 3.1.21. Now we can apply Theorem 1.6.1 to conclude that the last expression on the r.h.s. of (3.1.6) is bounded uniformly in $K$. As $|K|$ can be made arbitrarily large, it follows that

$$
\omega\left(A(f)^{*} A(f)\right)=0
$$

for any $A \in \mathfrak{A}(\mathcal{O})$ and $f$ as defined above. Since equality (3.1.7) extends to any $A \in \mathfrak{A}$, we can proceed with the proof that $\omega$ is a vacuum state similarly as in the proof of Theorem 4.5 of $\underline{\mathrm{Ar}}$ : First, one has to show that the functions

$$
\mathbb{R}^{s+1} \ni x \rightarrow \omega\left(A^{*} B(x)\right)
$$

are continuous for any $A, B \in \mathfrak{A}$. For local operators $A, B$ this follows from the local normality of the state $\omega$, ensured by Lemma 3.1.2 and the fact that the functions $\mathbb{R}^{s+1} \ni$ $x \rightarrow B(x)$ are continuous in the strong operator topology of $B(\mathcal{H})$. Since local operators are norm dense in $\mathfrak{A}$, we obtain continuity for all $A, B \in \mathfrak{A}$. In particular, $h(x)=\omega(B(x))$, $B \in \mathfrak{A}$, is continuous and bounded. Let us now choose any function $f \in S\left(\mathbb{R}^{s+1}\right)$ such that $\tilde{f}$ is compactly supported and $0 \notin \operatorname{supp} \tilde{f}$. Then it can be decomposed into a sum $f=f_{1}+f_{2}$ s.t. $\operatorname{supp} \tilde{f}_{1} \cap \bar{V}_{+}=\emptyset$ and supp $\tilde{\bar{f}}_{2} \cap \bar{V}_{+}=\emptyset$. Consequently, by the CauchySchwarz inequality and relation (3.1.7)

$$
\int h(x) f(x) d^{s+1} x=\omega\left(B\left(f_{1}\right)\right)+\overline{\omega\left(B^{*}\left(\bar{f}_{2}\right)\right)}=0,
$$

showing that $\operatorname{supp} \tilde{h} \subset\{0\}$. This implies that $h(x)=$ const, what entails the translational invariance of the state $\omega$. It follows that translations are unitarily implemented in the GNSrepresentation $\left(\mathcal{H}_{\omega}, \pi_{\omega}, \Omega_{\omega}\right)$ induced by $\omega$. The resulting unitary representation $\mathbb{R}^{s+1} \ni$ $x \rightarrow U_{\omega}(x)$, acting on $\mathcal{H}_{\omega}$, is strongly continuous by the continuity of the functions (3.1.8). To verify the spectral condition, we choose $f \in S\left(\mathbb{R}^{s+1}\right)$, s.t. $\operatorname{supp} \tilde{f} \cap \bar{V}_{+}=\emptyset, A, B \in \mathfrak{A}$ and calculate

$$
\begin{array}{r}
\left|\int\left(\pi_{\omega}(A) \Omega_{\omega} \mid U_{\omega}(x) \pi_{\omega}(B) \Omega\right) f(x) d^{s+1} x\right|^{2}=|\omega(A B(f))|^{2} \\
\leq \omega\left(A^{*} A\right) \omega\left(B(f)^{*} B(f)\right)=0,
\end{array}
$$

where we made use again of identity (3.1.7).

Making use of the above theorem, we can construct vacuum states with the help of the method of 'large translations' envisaged first in BHS63. The case of spacelike translations is well known [BP90, although our proof, based on Theorem 3.1.3 appears to be new.

Proposition 3.1.4. Suppose that Condition $C_{\sharp}$ holds and let $\hat{e} \in \mathbb{R}^{s+1}$ be a spacelike unit vector. Then there exists a net $\left\{\lambda_{\beta} \in \mathbb{R} \mid \beta \in \mathbb{I}\right\}$ s.t. $\lambda_{\beta} \rightarrow \infty$ and a vacuum state $\omega_{0} \in \mathfrak{A}^{*}$ s.t. for any $A \in \mathfrak{A}$ there holds

$$
\mathrm{w}^{*}-\lim _{\beta} A\left(\lambda_{\beta} \hat{e}\right)=\omega_{0}(A) I,
$$

where the limit is taken in the weak* topology of $B(\mathcal{H})$. 
Proof. Noting that $\left\{A\left(\lambda_{\beta} \hat{e}\right)\right\}_{\beta \in \mathbb{I}}$ is a central net in $\mathfrak{A}$, and proceeding identically as in the proof of Proposition 2.2.1 one obtains that relation (3.1.11) holds for some translationally invariant state $\omega_{0}$ which belongs to the closure of $\mathcal{T}_{E, 1}$, for any $E \geq 0$, in the weak* topology of $\mathfrak{A}^{*}$. Now making use of Theorem 3.1 .3 we conclude that $\omega_{0}$ is a vacuum state.

Now we turn to the more interesting problem of convergence of physical states to the vacuum state under large timelike translations. Here we cannot exploit locality directly, but instead we rely on Lorentz transformations. We need the following regularity assumption on their action $L_{+}^{\uparrow} \ni \Lambda \rightarrow \alpha_{\Lambda}$ on the global algebra $\mathfrak{A}$.

Condition $R$ : Let $\omega \in \mathcal{T}$. Then, for any open bounded region $\mathcal{O}$ and any timelike unit vector $\hat{e}$ there holds

$$
\sup _{\lambda \in \mathbb{R}_{+}} \sup _{A \in \mathfrak{A}(\mathcal{O}+\lambda \hat{e})_{1}}\left|\alpha_{\Lambda}^{*} \omega(A)-\omega(A)\right| \underset{\Lambda \rightarrow I}{\longrightarrow} 0 .
$$

This condition is satisfied, in particular, if the Lorentz transformations are unitarily implemented. The following result is based on the observation due to Buchholz that the timelike limit points of physical states are invariant under translations in some spacelike hyperplane.

Proposition 3.1.5. Suppose that Conditions $C_{\sharp}$ and $R$ hold. Let $\omega_{0}$ be a weak limit $^{*}$ point as $\lambda \rightarrow \infty$ of the net $\left\{\alpha_{\lambda \hat{e}}^{*} \omega\right\}_{\lambda \in \mathbb{R}_{+}}$of states on $\mathfrak{A}$, where $\hat{e} \in \mathbb{R}^{s+1}$ is a timelike unit vector and $\omega$ is a state from $\mathcal{T}_{E}$ for some $E \geq 0$. Then $\omega_{0}$ is a vacuum state.

Proof. In view of Theorem 3.1.3 it suffices to show that $\omega_{0}$ is invariant under translations in the spacelike hyperplane $\left\{\hat{e}^{\perp}\right\}=\left\{x \in \mathbb{R}^{s+1} \mid \hat{e} \cdot x=0\right\}$, where the dot denotes the Minkowski scalar product.

Choose $x \in\left\{\hat{e}^{\perp}\right\}, x \neq 0$. Then there exists a Lorentz transformation $\Lambda$ and $y^{0}, y^{1} \in \mathbb{R} \backslash\{0\}$ s.t. $\Lambda \hat{e}=y^{0} \hat{e}_{0}, \Lambda x=y^{1} \hat{e}_{1}$, where $\hat{e}_{\mu}, \mu \in\{0,1, \ldots, s\}$ form the canonical basis in $\mathbb{R}^{s+1}$. We set $v=\frac{y^{1}}{y^{0}}$ and introduce the family of Lorentz transformations $\Lambda_{t}=\Lambda^{-1} \tilde{\Lambda}_{t} \Lambda$, where $\tilde{\Lambda}_{t}$ denotes the boost in the direction of $\hat{e}_{1}$ with rapidity $\operatorname{arsinh}\left(\frac{v}{t}\right)$. By the composition law of the Poincaré group, the above transformations composed with translations in timelike direction give also rise to spacelike translations

$$
\left(0, \Lambda_{\lambda}\right)(\lambda \hat{e}, I)\left(0, \Lambda_{\lambda}^{-1}\right)=\left(\lambda \Lambda_{\lambda} \hat{e}, I\right), \quad \lambda \Lambda_{\lambda} \hat{e}=\lambda \sqrt{1+(v / \lambda)^{2}} \hat{e}+x .
$$

We make use of this fact in the following estimate

$$
\begin{aligned}
\left|\alpha_{\lambda \hat{e}}^{*} \omega(A)-\alpha_{\lambda \hat{e}}^{*} \omega(A(x))\right| & \leq\left|\omega\left(\alpha_{\lambda \hat{e}} A\right)-\omega\left(\alpha_{\Lambda_{\lambda}} \alpha_{\lambda \hat{e}} \alpha_{\Lambda_{\lambda}^{-1}} A\right)\right| \\
& +\left|\alpha_{\lambda \Lambda_{\lambda} \hat{e}}^{*} \omega(A)-\alpha_{\lambda \hat{e}}^{*} \omega(A(x))\right|,
\end{aligned}
$$

where $A \in \mathfrak{A}(\mathcal{O})$. The first term on the r.h.s. of (3.1.14) satisfies the bound

$$
\begin{aligned}
& \left|\omega\left(\alpha_{\lambda \hat{e}} A\right)-\omega\left(\alpha_{\Lambda_{\lambda}} \alpha_{\lambda \hat{e}} \alpha_{\Lambda_{\lambda}^{-1}} A\right)\right| \\
& \quad \leq\left|\alpha_{\lambda \hat{e}}^{*} \omega\left(A-\alpha_{\Lambda_{\lambda}^{-1}} A\right)\right|+\left|\left(\omega-\alpha_{\Lambda_{\lambda}}^{*} \omega\right)\left(\alpha_{\lambda \hat{e}} \alpha_{\Lambda_{\lambda}^{-1}} A\right)\right| \\
& \quad \leq\left\|P_{E}\left(A-\alpha_{\Lambda_{\lambda}^{-1}} A\right) P_{E}\right\|+\sup _{s \in \mathbb{R}_{+}} \sup _{B \in \mathfrak{A}(\widetilde{\mathcal{O}}+s \hat{e})_{1}}\left|\omega(B)-\alpha_{\Lambda_{\lambda}}^{*} \omega(B)\right|\|A\|,
\end{aligned}
$$


where $\widetilde{\mathcal{O}}$ is a slightly larger region than $\mathcal{O}$. Making use of Condition $R$ and of the fact that $\Lambda_{\lambda} \rightarrow I$ for $\lambda \rightarrow \infty$, we obtain that the last term above tends to zero in this limit. In view of our general continuity assumption on the group of automorphisms $\alpha$ from Section [1.6, the expression $\left(A-\alpha_{\Lambda_{\lambda}^{-1}} A\right)$ tends to zero with $\lambda \rightarrow \infty$ in the strong operator topology. By the compactness of the maps $\Theta_{E}$, the first term on the r.h.s. of (3.1.15) tends to zero as well. The second term on the r.h.s. of (3.1.14) converges to zero by the compactness of the maps $\Theta_{E}$ and the following bound

$$
\begin{array}{r}
\left|\alpha_{\lambda \Lambda_{\lambda} \hat{e}}^{*} \omega(A)-\alpha_{\lambda \hat{e}}^{*} \omega(A(x))\right|=\left|\omega\left(A\left(\lambda \sqrt{1+(v / \lambda)^{2}} \hat{e}+x\right)-A(\lambda \hat{e}+x)\right)\right| \\
\leq\left\|P_{E}\left(A\left(\left\{\sqrt{1+(v / \lambda)^{2}}+1\right\}^{-1}\left(v^{2} / \lambda\right) \hat{e}\right)-A\right) P_{E}\right\| .
\end{array}
$$

Thus we have demonstrated that $\omega_{0}(A)=\omega_{0}(A(x))$ for any local operator $A$. This result extends by continuity to any $A \in \mathfrak{A}$.

Summing up, we have shown that there exist vacuum states in any theory satisfying Condition $C_{\sharp}$. These vacuum states can be constructed by means of large spacelike or timelike translations of physical states. (It is an interesting open question if this result holds also for lightlike directions). However, the uniqueness of the energetically accessible vacuum state does not seem to follow from this criterion, since the concept of compactness is compatible with many limit points. Our next task is to find a quantitative variant of Condition $C_{\sharp}$ which entails also this property. To this end, in the next section we cast this criterion in a form appropriate for a description of coincidence measurements. Then, in Section 3.3. we propose a strengthened, quantitative form of Condition $C_{\sharp}$. It accounts for the fact that if the number of separated observables, with vanishing vacuum expectation values, is larger than the number of localization centers forming the state under study, then the result of the coincidence measurement should be zero.

\subsection{Condition $C_{\sharp}$ : Coincidence Measurement Formulation}

To formulate a notion of compactness which is adequate for a description of coincidence arrangements of detectors, we need to extend our framework: Let $\Gamma$ be a set and let $\mathcal{L}(V \times \Gamma, W)$ be the space of maps $\Pi$ from $V \times \Gamma$ to $W$, linear in the first argument, which are bounded in the norm

$$
\|\Pi\|=\sup _{\substack{v \in V_{1} \\ x \in \Gamma}}\|\Pi(v, x)\| .
$$

The subspace of finite rank maps $\mathcal{F}(V \times \Gamma, W)$ contains all the maps of the form $F=$ $\sum_{i=1}^{n} \tau_{i} S_{i}$, where $\tau_{i} \in W, S_{i} \in \mathcal{L}(V \times \Gamma, \mathbb{C})$. We say that a map $\Pi \in \mathcal{L}(V \times \Gamma, W)$ is compact, if it belongs to the closure of $\mathcal{F}(V \times \Gamma, W)$ in the norm topology of $\mathcal{L}(V \times \Gamma, W)$.

As we are going to consider coincidence measurements, we choose as the target space $W$ the Banach space $\left(\mathfrak{A}(\mathcal{O})^{\times N}\right)^{*}$ of $N$-linear forms on $\mathfrak{A}(\mathcal{O})$ equipped with the norm

$$
\|\psi\|=\sup _{\substack{A_{i} \in \mathfrak{A}(\mathcal{O})_{1} \\ i \in\{1, \ldots, N\}}}\left|\psi\left(A_{1} \times \cdots \times A_{N}\right)\right| .
$$

In order to control the minimal distance between the regions in which the measurements are performed, we define the set of admissible translates of the region $\mathcal{O}$

$$
\Gamma_{N, \delta}=\left\{\underline{\vec{x}}=\left(\vec{x}_{1}, \ldots, \vec{x}_{N}\right) \in \mathbb{R}^{N s} \mid \forall_{t \in]-\delta, \delta[, i \neq j} \mathcal{O}+\vec{x}_{i} \sim \mathcal{O}+\vec{x}_{j}+t \hat{e}_{0}\right\},
$$


where the symbol $\sim$ indicates spacelike separation and $\hat{e}_{0}$ is the unit vector in the time direction. For any $\underline{\vec{x}} \in \Gamma_{N, \delta}$ and $\varphi \in \mathcal{T}_{E}$ we introduce the following elements of $\left(\mathfrak{A}(\mathcal{O})^{\times N}\right)^{*}$

$$
\varphi_{\underline{\vec{x}}}\left(A_{1} \times \cdots \times A_{N}\right)=\varphi\left(A_{1}\left(\vec{x}_{1}\right) \ldots A_{N}\left(\vec{x}_{N}\right)\right),
$$

and consider the maps $\Pi_{E, N, \delta} \in \mathcal{L}\left(\mathcal{T}_{E} \times \Gamma_{N, \delta},\left(\mathfrak{A}(\mathcal{O})^{\times N}\right)^{*}\right)$ given by

$$
\Pi_{E, N, \delta}(\varphi, \underline{\vec{x}})=\varphi_{\underline{\vec{x}}} .
$$

The following theorem provides a reformulation of Condition $C_{\sharp}$ in terms of these maps.

Theorem 3.2.1. A theory satisfies Condition $C_{\sharp}$ if and only if the maps $\Pi_{E, N, \delta}$ are compact for any $E \geq 0, N \in \mathbb{N}, \delta>0$ and any double cone $\mathcal{O}$.

The 'if' part of the statement holds due to the identity $\Pi_{E, 1, \delta}=\Pi_{E}$. The opposite implication is more interesting. It says that the restriction imposed by Condition $C_{\sharp}$ on the number of states which can be distinguished by measurements with singly-localized detectors limits also the number of states which can be discriminated by coincidence arrangements of such detectors. We start our analysis from the observation that the spatial distance between the detectors suppresses the energy transfer between them. The proof of the following lemma relies on methods from [BY87.

Lemma 3.2.2. Let $\delta>0, \beta>0$. Define the function $g:]-\pi, \pi] \rightarrow \mathbb{C}$ as follows

$$
g(\theta)=\frac{\beta}{\pi} \ln \left|\cot \frac{\theta+\gamma}{2} \cot \frac{\theta-\gamma}{2}\right|
$$

where $\gamma=2 \arctan e^{-\frac{\pi \delta}{2 \beta}}$. Then, for any pair of bounded operators $A, B$, satisfying $[A(t), B]=0$ for $|t|<\delta$, and any functional $\varphi \in e^{-\beta H} \mathcal{T} e^{-\beta H}$ there holds the identity

$$
\varphi(A B)=\varphi\left(\left[A, \stackrel{\circ}{B}_{\beta}\right]_{+}\right)+\varphi\left(A e^{-\beta H} B_{\beta} e^{\beta H}\right)+\varphi\left(e^{\beta H} B_{\beta} e^{-\beta H} A\right),
$$

where $[\cdot, \cdot]_{+}$denotes the anti-commutator and we made use of the fact that $\varphi\left(e^{\beta H} \cdot\right)$, $\varphi\left(\cdot e^{\beta H}\right)$ are elements of $\mathcal{T}$. Here $\stackrel{\circ}{B}_{\beta}$ and $B_{\beta}$ are elements of $B(\mathcal{H})$ given by the (weak) integrals

$$
\begin{aligned}
\stackrel{\circ}{B}_{\beta} & =\frac{1}{2 \pi} \int_{0}^{\gamma} d \theta B(g(\theta))+\frac{1}{2 \pi} \int_{\pi-\gamma}^{\pi} d \theta B(g(\theta)), \\
B_{\beta} & =\frac{1}{2 \pi} \int_{\gamma}^{\pi-\gamma} d \theta B(g(\theta)),
\end{aligned}
$$

where $B(g(\theta))=e^{i g(\theta) H} B e^{-i g(\theta) H}$.

Proof. It suffices to prove the statement for functionals of the form $\varphi(\cdot)=\left(\Psi_{1} \mid \cdot \Psi_{2}\right)$, where $\Psi_{1}$ and $\Psi_{2}$ are vectors from the domain of $e^{\beta H}$. For $\delta>0$ and $\beta>0$ we define the set

$$
G_{\beta, \delta}=\{z \in \mathbb{C}|| \operatorname{Im} z \mid<\beta\} \backslash\{z|\operatorname{Im} z=0,| \operatorname{Re} z \mid \geq \delta\}
$$

and introduce the following function, analytic on $G_{\beta, \delta}$ and continuous at its boundary

$$
h(z)= \begin{cases}\left(\Psi_{1} \mid A e^{i z H} B e^{-i z H} \Psi_{2}\right) & \text { for } 0<\operatorname{Im} z<\beta \\ \left(\Psi_{1} \mid e^{i z H} B e^{-i z H} A \Psi_{2}\right) & \text { for }-\beta<\operatorname{Im} z<0 \\ \left(\Psi_{1} \mid A B(z) \Psi_{2}\right)=\left(\Psi_{1} \mid B(z) A \Psi_{2}\right) & \text { for } \operatorname{Im} z=0 \text { and }|\operatorname{Re} z|<\delta .\end{cases}
$$


We make use of the following conformal mapping from the unit disc $\{w|| w \mid<1\}$ to $G_{\beta, \delta}$ BY87,

$$
z(w)=\frac{\beta}{\pi}\left\{\ln \frac{1+w e^{i \gamma}}{1-w e^{i \gamma}}-\ln \frac{1-w e^{-i \gamma}}{1+w e^{i \gamma}}\right\} .
$$

Setting $w=r e^{i \theta}, 0<r<1$, we obtain from the Cauchy formula

$$
h(0)=\frac{1}{2 \pi} \int_{0}^{2 \pi} d \theta h\left(z\left(r e^{i \theta}\right)\right) .
$$

Since $h(z)$ satisfies the following bound on the closure of $G_{\beta, \delta}$

$$
|h(z)| \leq\|A\|\|B\|\left\|e^{\beta H} \Psi_{1}\right\|\left\|e^{\beta H} \Psi_{2}\right\|,
$$

we can, by the dominated convergence theorem, extend the path of integration in (3.2.13) to the circle $r=1$. In this limit we have BY87]

$$
\begin{aligned}
& \operatorname{Re} z\left(e^{i \theta}\right)=g(\theta), \\
& \operatorname{Im} z\left(e^{i \theta}\right)= \begin{cases}0 & \text { if }|\theta|<\gamma \text { or } \pi-\theta<\gamma \text { or } \pi+\theta<\gamma \\
\beta & \text { if } \gamma<\theta<\pi-\gamma \\
-\beta & \text { if } \gamma<-\theta<\pi-\gamma .\end{cases}
\end{aligned}
$$

Consequently, we obtain from (3.2.13)

$$
\begin{aligned}
& \left(\Psi_{1} \mid A B \Psi_{2}\right) \\
= & \frac{1}{2 \pi} \int_{0}^{\gamma} d \theta\left(\Psi_{1} \mid[A, B(g(\theta))]_{+} \Psi_{2}\right)+\frac{1}{2 \pi} \int_{\pi-\gamma}^{\pi} d \theta\left(\Psi_{1} \mid[A, B(g(\theta))]_{+} \Psi_{2}\right) \\
+ & \frac{1}{2 \pi} \int_{\gamma}^{\pi-\gamma} d \theta\left(\left(\Psi_{1} \mid A e^{-\beta H} B(g(\theta)) e^{\beta H} \Psi_{2}\right)+\left(e^{\beta H} \Psi_{1} \mid B(g(\theta)) e^{-\beta H} A \Psi_{2}\right)\right),
\end{aligned}
$$

what concludes the proof.

From the compactness of the maps $\Xi_{\beta}$, given by (3.1.3), there also follows that the mappings $\Xi_{\beta_{1}, \beta_{2}}: \mathfrak{A}(\mathcal{O}) \rightarrow B(\mathcal{H})$, defined as

$$
\Xi_{\beta_{1}, \beta_{2}}(A)=e^{-\beta_{1} H} A_{\beta_{2}} e^{-\beta_{1} H},
$$

are compact for any $\beta_{1}, \beta_{2}>0$ and any double cone $\mathcal{O}$. Here $A_{\beta_{2}}$ is defined as in (3.2.9). After this preparation we are ready to complete the proof of Theorem 3.2.1

Proof of Theorem 3.2.1 For any $\beta>0$ we introduce the auxiliary maps $\widehat{\Pi}_{\beta, N, \delta} \in$ $\mathcal{L}\left(\mathcal{T} \times \Gamma_{N, \delta},\left(\mathfrak{A}(\mathcal{O})^{\times N}\right)^{*}\right)$ given by

$$
\widehat{\Pi}_{\beta, N, \delta}(\varphi, \underline{\vec{x}})\left(A_{1} \times \cdots \times A_{N}\right)=\varphi\left(e^{-\left(N+\frac{1}{2}\right) \beta H} A_{1}\left(\vec{x}_{1}\right) \ldots A_{N}\left(\vec{x}_{N}\right) e^{-\left(N+\frac{1}{2}\right) \beta H}\right) .
$$

They are related to the maps $\Pi_{E, N, \delta} \in \mathcal{L}\left(\mathcal{T}_{E} \times \Gamma_{N, \delta},\left(\mathfrak{A}(\mathcal{O})^{\times N}\right)^{*}\right)$ by the following identity, valid for any $\varphi \in \mathcal{T}_{E}$,

$$
\Pi_{E, N, \delta}(\varphi, \underline{\vec{x}})=\widehat{\Pi}_{\beta, N, \delta}\left(e^{\left(N+\frac{1}{2}\right) \beta H} \varphi e^{\left(N+\frac{1}{2}\right) \beta H}, \underline{\vec{x}}\right) .
$$

In order to prove the compactness of the maps $\Pi_{E, N, \delta}$, it suffices to verify that the family of mappings $\left\{\widehat{\Pi}_{\beta, N, \delta}\right\}_{\beta>0}$ is asymptotically compact in the following sense: There exists a family of finite rank maps $\widehat{F}_{\beta, N, \delta} \in \mathcal{F}\left(\mathcal{T} \times \Gamma_{N, \delta},\left(\mathfrak{A}(\mathcal{O})^{\times N}\right)^{*}\right)$ s.t.

$$
\lim _{\beta \rightarrow 0}\left\|\widehat{\Pi}_{\beta, N, \delta}-\widehat{F}_{\beta, N, \delta}\right\|=0 .
$$


If this property holds, then, by identity (3.2.20), the maps $\Pi_{E, N, \delta}$ can be approximated in norm as $\beta \rightarrow 0$ by the finite rank maps $F_{\beta, N, \delta} \in \mathcal{F}\left(\mathcal{T}_{E} \times \Gamma_{N, \delta},\left(\mathfrak{A}(\mathcal{O})^{\times N}\right)^{*}\right)$ defined as

$$
F_{\beta, N, \delta}(\varphi, \underline{\vec{x}})=\widehat{F}_{\beta, N, \delta}\left(e^{\left(N+\frac{1}{2}\right) \beta H} \varphi e^{\left(N+\frac{1}{2}\right) \beta H}, \underline{\vec{x}}\right) .
$$

We establish property (3.2.21) by induction in $N$ : For $N=1$ the statement follows from the compactness of the map $\Xi_{\frac{3}{2} \beta}$ given by (3.1.2). Next, we assume that the family $\left\{\widehat{\Pi}_{\beta, N-1, \delta}\right\}_{\beta>0}$ is asymptotically compact and prove that $\left\{\widehat{\Pi}_{\beta, N, \delta}\right\}_{\beta>0}$ also has this property. For this purpose we pick $\varphi \in \mathcal{T}_{1}, A_{1}, \ldots, A_{N} \in \mathfrak{A}(\mathcal{O})_{1}$ and $\underline{\vec{x}} \in \Gamma_{N, \delta}$. Then $A_{1}\left(\vec{x}_{1}\right) \ldots A_{N-1}\left(\vec{x}_{N-1}\right)$ and $A_{N}\left(\vec{x}_{N}\right)$ satisfy the assumptions of Lemma 3.2.2 and we obtain

$$
\begin{aligned}
& \widehat{\Pi}_{\beta, N, \delta}(\varphi, \underline{\vec{x}})\left(A_{1} \times \cdots \times A_{N}\right) \\
& =\varphi\left(e^{-\left(N+\frac{1}{2}\right) \beta H}\left[A_{1}\left(\vec{x}_{1}\right) \ldots A_{N-1}\left(\vec{x}_{N-1}\right), \stackrel{A}{N, N \beta}\left(\vec{x}_{N}\right)\right]_{+} e^{-\left(N+\frac{1}{2}\right) \beta H}\right) \\
& +\widehat{\Pi}_{\beta, N-1, \delta}\left(\left\{\Xi_{\frac{1}{2} \beta, N \beta}\left(A_{N}\right)\left(\vec{x}_{N}\right) \varphi e^{-\frac{1}{2} \beta H}\right\}, \vec{x}_{1}, \ldots, \vec{x}_{N-1}\right)\left(A_{1} \times \cdots \times A_{N-1}\right) \\
& +\widehat{\Pi}_{\beta, N-1, \delta}\left(\left\{e^{-\frac{1}{2} \beta H} \varphi \Xi_{\frac{1}{2} \beta, N \beta}\left(A_{N}\right)\left(\vec{x}_{N}\right)\right\}, \vec{x}_{1}, \ldots, \vec{x}_{N-1}\right)\left(A_{1} \times \cdots \times A_{N-1}\right),(3.2
\end{aligned}
$$

where $[\cdot, \cdot]_{+}$denotes the anti-commutator. The first term on the r.h.s. of (3.2.23) satisfies

$$
\left|\varphi\left(e^{-\left(N+\frac{1}{2}\right) \beta H}\left[A_{1}\left(\vec{x}_{1}\right) \ldots A_{N-1}\left(\vec{x}_{N-1}\right), \AA_{N, N \beta}\left(\vec{x}_{N}\right)\right]_{+} e^{-\left(N+\frac{1}{2}\right) \beta H}\right)\right| \leq \frac{2 \gamma(\beta)}{\pi},
$$

where we made use of definition (3.2.8). We recall from the statement of Lemma 3.2.2 that $\gamma(\beta) \rightarrow 0$ with $\beta \rightarrow 0$. To treat the remaining terms, we make use of the induction hypothesis. It assures that there exist finite rank mappings $\widehat{F}_{\beta, N-1, \delta} \in \mathcal{F}(\mathcal{T} \times$ $\left.\Gamma_{N-1, \delta},\left(\mathfrak{A}(\mathcal{O})^{\times N-1}\right)^{*}\right)$ s.t.

$$
\lim _{\beta \rightarrow 0}\left\|\widehat{\Pi}_{\beta, N-1, \delta}-\widehat{F}_{\beta, N-1, \delta}\right\|=0 .
$$

Next, making use of the compactness of the maps $\Xi_{\frac{1}{2} \beta, N \beta} \in \mathcal{L}(\mathfrak{A}(\mathcal{O}), B(\mathcal{H}))$, given by (3.2.18), we can find a family of finite rank mappings $F_{\beta} \in \mathcal{F}(\mathfrak{A}(\mathcal{O}), B(\mathcal{H}))$ s.t.

$$
\left\|\Xi_{\frac{1}{2} \beta, N \beta}-F_{\beta}\right\| \leq \frac{\beta}{1+\left\|\widehat{F}_{\beta, N-1, \delta}\right\|}
$$

for any $\beta>0$. Now the second term on the r.h.s. of (3.2.23) can be rewritten as follows

$$
\begin{aligned}
\widehat{\Pi}_{\beta, N-1, \delta}\left(\left\{\Xi_{\frac{1}{2} \beta, N \beta}\left(A_{N}\right)\left(\vec{x}_{N}\right) \varphi e^{-\frac{1}{2} \beta H}\right\}, \vec{x}_{1}, \ldots, \vec{x}_{N-1}\right) \\
\quad=\left(\widehat{\Pi}_{\beta, N-1, \delta}-\widehat{F}_{\beta, N-1, \delta}\right)\left(\left\{\Xi_{\frac{1}{2} \beta, N \beta}\left(A_{N}\right)\left(\vec{x}_{N}\right) \varphi e^{-\frac{1}{2} \beta H}\right\}, \vec{x}_{1}, \ldots, \vec{x}_{N-1}\right) \\
\quad+\widehat{F}_{\beta, N-1, \delta}\left(\left\{\left(\Xi_{\frac{1}{2} \beta, N \beta}\left(A_{N}\right)-F_{\beta}\left(A_{N}\right)\right)\left(\vec{x}_{N}\right) \varphi e^{-\frac{1}{2} \beta H}\right\}, \vec{x}_{1}, \ldots, \vec{x}_{N-1}\right) \\
\quad+\widehat{F}_{\beta, N-1, \delta}\left(\left\{F_{\beta}\left(A_{N}\right)\left(\vec{x}_{N}\right) \varphi e^{-\frac{1}{2} \beta H}\right\}, \vec{x}_{1}, \ldots, \vec{x}_{N-1}\right) .
\end{aligned}
$$

We obtain from relations (3.2.25) and (3.2.26) that the first two terms on the r.h.s. of (3.2.27) tend to zero with $\beta \rightarrow 0$ in the norm topology of $\left(\mathfrak{A}(\mathcal{O})^{\times N-1}\right)^{*}$, uniformly in 
$\varphi \in \mathcal{T}_{1}, A_{N} \in \mathfrak{A}(\mathcal{O})_{1}$ and $\underline{\vec{x}} \in \Gamma_{N, \delta}$. The last term on the r.h.s. of (3.2.27) coincides with the finite rank map $\widehat{F}_{\beta, N, \delta}^{(1)} \in \mathcal{F}\left(\mathcal{T} \times \Gamma_{N, \delta},\left(\mathfrak{A}(\mathcal{O})^{\times N}\right)^{*}\right)$, given by

$$
\begin{aligned}
& \widehat{F}_{\beta, N, \delta}^{(1)}(\varphi, \vec{x})\left(A_{1} \times \cdots \times A_{N}\right) \\
& \quad=\widehat{F}_{\beta, N-1, \delta}\left(\left\{F_{\beta}\left(A_{N}\right)\left(\vec{x}_{N}\right) \varphi e^{-\frac{1}{2} \beta H}\right\}, \vec{x}_{1}, \ldots, \vec{x}_{N-1}\right)\left(A_{1} \times \cdots \times A_{N-1}\right) .
\end{aligned}
$$

The last term on the r.h.s. of (3.2.23) can be analogously approximated by the maps $\widehat{F}_{\beta, N, \delta}^{(2)} \in \mathcal{F}\left(\mathcal{T} \times \Gamma_{N, \delta},\left(\mathfrak{A}(\mathcal{O})^{\times N}\right)^{*}\right)$ defined as

$$
\begin{aligned}
& \widehat{F}_{\beta, N, \delta}^{(2)}(\varphi, \vec{x})\left(A_{1} \times \cdots \times A_{N}\right) \\
& \quad=\widehat{F}_{\beta, N-1, \delta}\left(\left\{e^{-\frac{1}{2} \beta H} \varphi F_{\beta}\left(A_{N}\right)\left(\vec{x}_{N}\right)\right\}, \vec{x}_{1}, \ldots, \vec{x}_{N-1}\right)\left(A_{1} \times \cdots \times A_{N-1}\right) .
\end{aligned}
$$

Summing up, we obtain from (3.2.23) and (3.2.24) that

$$
\lim _{\beta \rightarrow 0}\left\|\widehat{\Pi}_{\beta, N, \delta}-\widehat{F}_{\beta, N, \delta}^{(1)}-\widehat{F}_{\beta, N, \delta}^{(2)}\right\|=0,
$$

what concludes the inductive argument and the proof of Theorem 3.2 .1

\subsection{Condition $C_{\mathrm{b}}$ and Uniqueness of Vacuum}

Theorem 3.2.1 opens the possibility to encode the physically expected behavior of coincidence arrangement of detectors into the phase space structure of a theory. For this purpose we first calibrate the observables appropriately: Adopting Condition $C_{\sharp}$, from Proposition 2.2.1 and Theorem 3.1.3 we obtain a projection $P_{\{0\}}(\cdot)=\omega_{0}(\cdot) I$ on the pure-point subspace $\hat{\mathfrak{A}}_{\text {pp }}$, where $\omega_{0}$ is some energetically accessible vacuum state. As in Section [2.2. we define the continuous subspace $\hat{\mathfrak{A}}_{\mathrm{c}}$ as the kernel of $P_{\{0\}}$ and introduce, for any open, bounded region $\mathcal{O}$, the local continuous subspace

$$
\mathfrak{A}_{\mathrm{c}}(\mathcal{O})=\left\{A \in \mathfrak{A}(\mathcal{O}) \mid \omega_{0}(A)=0\right\},
$$

whose elements will be called the local detectors. We introduce the Banach space $\left(\mathfrak{A}_{\mathrm{c}}(\mathcal{O})^{\times N}\right)^{*}$ of $N$-linear forms on $\mathfrak{A}_{\mathrm{c}}(\mathcal{O})$ equipped with the norm

$$
\|\psi\|=\sup _{\substack{A_{i} \in \mathfrak{A}_{\mathrm{C}}(\mathcal{O})_{1} \\ i \in\{1, \ldots, N\}}}\left|\psi\left(A_{1} \times \cdots \times A_{N}\right)\right| .
$$

We also define the maps $\Pi_{E, N, \delta}^{\mathrm{c}} \in \mathcal{L}\left(\mathcal{T}_{E} \times \Gamma_{N, \delta},\left(\mathfrak{A}_{\mathrm{c}}(\mathcal{O})^{\times N}\right)^{*}\right)$ given by

$$
\Pi_{E, N, \delta}^{\mathrm{c}}(\varphi, \underline{\vec{x}})=\left.\Pi_{E, N, \delta}(\varphi, \underline{\vec{x}})\right|_{\mathfrak{A}_{\mathrm{C}}(\mathcal{O}) \times N} .
$$

With the help of Theorem 3.2 .1 we can reformulate Condition $C_{\sharp}$ in terms of these maps.

Theorem 3.3.1. A theory satisfies Condition $C_{\sharp}$ if and only if there exists an energetically accessible vacuum state $\omega_{0}$ and the corresponding maps $\Pi_{E, N, \delta}^{\mathrm{c}}$ are compact for any $E \geq 0$, $N \in \mathbb{N}, \delta>0$ and any double cone $\mathcal{O}$. 
Proof. The 'only if' part follows immediately from Theorem 3.2.1. The opposite implication can be shown as follows: From the compactness of the map $\Pi_{E, 1, \delta}^{\mathrm{c}}$ we obtain, for any $\varepsilon>0$, a finite rank map $F \in \mathcal{F}\left(\mathcal{T}_{E} \times \mathbb{R}^{s}, \mathfrak{A}_{\mathrm{c}}(\mathcal{O})^{*}\right)$ s.t.

$$
\sup _{\substack{(\varphi, \vec{x}) \in \mathcal{T}_{E, 1 \times \mathbb{R}^{s}} \\ A \in \mathfrak{A}_{c}(\mathcal{O})_{1}}}\left|\Pi_{E, 1, \delta}^{\mathrm{c}}(\varphi, \vec{x})(A)-F(\varphi, \vec{x})(A)\right| \leq \varepsilon .
$$

Noting that $\Pi_{E, 1, \delta}^{\mathrm{c}}(\varphi, \vec{x})=\left.\Pi_{E}\left(\varphi_{\vec{x}}\right)\right|_{\mathfrak{A}_{c}(\mathcal{O})}$ and making use of the fact that $\frac{1}{2}\left(A-\omega_{0}(A) I\right) \in$ $\mathfrak{A}_{\mathrm{c}}(\mathcal{O})_{1}$ for any $A \in \mathfrak{A}(\mathcal{O})$, we obtain

$$
\sup _{\substack{\varphi \in \mathcal{T}_{E, 1} \\ A \in \mathfrak{A}(\mathcal{O})_{1}}}\left|\Pi_{E}(\varphi)(A)-\varphi(I) \omega_{0}(A)-F(\varphi, 0)\left(A-\omega_{0}(A) I\right)\right| \leq 2 \varepsilon .
$$

Thus we can approximate the maps $\Pi_{E}$ in norm with finite rank mappings i.e. these maps are compact.

In order to strengthen Condition $C_{\sharp}$, we note that any functional from $\mathcal{T}_{E}$ should consist of a finite number of distinct localization centers. Indeed, in a theory describing particles of mass $m>0$ the maximal number of such centers $N_{0}(E)$ is given, essentially, by $\frac{E}{m}$. If the number of detectors $N$ is larger than $N_{0}(E)$, then at least one of them should give no response (since its vacuum expectation value vanishes) and the result of the entire coincidence measurement should be zero.

In order to formulate this observation mathematically, we adapt the concept of the $\varepsilon$ content to the present framework: Let $V, W$ be Banach spaces and let $\Gamma$ be some set. Then the $\varepsilon$-content of a map $\Pi \in \mathcal{L}(V \times \Gamma, W)$ is the maximal natural number $\mathcal{N}(\varepsilon)$ for which there exist elements $\left(v_{1}, x_{1}\right), \ldots,\left(v_{\mathcal{N}(\varepsilon)}, x_{\mathcal{N}(\varepsilon)}\right) \in V_{1} \times \Gamma$ s.t. $\left\|\Pi\left(v_{i}, x_{i}\right)-\Pi\left(v_{j}, x_{j}\right)\right\|>\varepsilon$ for $i \neq j$. Clearly, this quantity is finite if the map $\Pi$ is compact.

The $\varepsilon$-content $\mathcal{N}(\varepsilon)_{E, N, \delta}^{\mathrm{c}}$ of the map $\Pi_{E, N, \delta}^{\mathrm{c}}$ gives the number of distinct measurement results, up to experimental accuracy $\varepsilon$, which can be obtained in the coincidence measurement described above. Therefore, we expect that $\mathcal{N}(\varepsilon)_{E, N, \delta}^{\mathrm{c}}=1$ if $N$ is sufficiently large and the local detectors are far apart, arriving at the following strengthened, quantitative variant of Condition $C_{\sharp}$ :

\section{Condition $C_{\mathrm{b}}$}

(a) There exists an energetically accessible vacuum state $\omega_{0}$ and the corresponding maps $\Pi_{E, N, \delta}^{\mathrm{c}}$ are compact for any $E \geq 0, N \in \mathbb{N}, \delta>0$ and any double cone $\mathcal{O}$.

(b) For any $E \geq 0$ there exists $N_{0}(E) \in \mathbb{N}$ s.t. for any $N>N_{0}(E)$ the $\varepsilon$-contents $\mathcal{N}(\varepsilon)_{E, N, \delta}^{\mathrm{c}}$ of the maps $\Pi_{E, N, \delta}^{\mathrm{c}}$ satisfy

$$
\lim _{\delta \rightarrow \infty} \mathcal{N}(\varepsilon)_{E, N, \delta}^{\mathrm{c}}=1 \quad \text { for any } \quad \varepsilon>0 .
$$

We verify in Appendix $[$ that this condition holds in massive scalar free field theory and its even part.

In the remaining part of this section we show that the vacuum state $\omega_{0}$, which entered into the formulation of Condition $C_{\mathrm{b}}$, is pure and that it is the only energetically accessible vacuum state. Then, from Propositions 3.1.4 and 3.1.5 there follows the convergence of physical states to $\omega_{0}$ under large spacelike and timelike translations. Moreover, 
we demonstrate that this vacuum state appears as a limit of states of increasingly sharp energy-momentum values. To achieve these goals it is convenient to reformulate Condition $C_{\mathrm{b}}$ (b) in terms of norms of the maps $\Pi_{E, N, \delta}$. This is accomplished in the following lemma.

Lemma 3.3.2. Let $W$ be a Banach space. Let $\left\{V_{\delta}\right\}_{\delta>0}$ and $\left\{\Gamma_{\delta}\right\}_{\delta>0}$ be a family of Banach spaces and sets, respectively, ordered by inclusion i.e. $V_{\delta_{1}} \subset V_{\delta_{2}}$ and $\Gamma_{\delta_{1}} \subset \Gamma_{\delta_{2}}$ for $\delta_{1} \geq \delta_{2}$. We consider a family $\left\{\Pi_{\delta}\right\}_{\delta>0}$ of compact maps in $\mathcal{L}\left(V_{\delta} \times \Gamma_{\delta}, W\right)$ and their respective $\varepsilon$-contents $\mathcal{N}(\varepsilon)_{\delta}$. Then there holds $\lim _{\delta \rightarrow \infty} \mathcal{N}(\varepsilon)_{\delta}=1$ for any $\varepsilon>0$ if and only if $\lim _{\delta \rightarrow \infty}\left\|\Pi_{\delta}\right\|=0$.

Proof. First, suppose that $\lim _{\delta \rightarrow \infty} \mathcal{N}(\varepsilon)_{\delta}=1$ for any $\varepsilon>0$. Since the $\varepsilon$-content takes only integer values, for any $\varepsilon>0$ we can choose $\delta_{\varepsilon}$ s.t. $\mathcal{N}(\varepsilon)_{\delta}=1$ for $\delta \geq \delta_{\varepsilon}$. Then, by definition of the $\varepsilon$-content, there holds for any $\delta \geq \delta_{\varepsilon}$

$$
\left\|\Pi_{\delta}\right\| \leq \varepsilon,
$$

what entails $\lim _{\delta \rightarrow \infty}\left\|\Pi_{\delta}\right\|=0$.

To prove the opposite implication, we proceed by contradiction: We recall that the $\varepsilon$-content of a compact map is finite for any $\varepsilon>0$. Next, we note that for any fixed $\varepsilon>0$ the function $\delta \rightarrow \mathcal{N}(\varepsilon)_{\delta}$ is decreasing and bounded from below by one, so there exists $\lim _{\delta \rightarrow \infty} \mathcal{N}(\varepsilon)_{\delta}$. Suppose that this limit is strictly larger than one. Then, by definition of the $\varepsilon$-content, there exist nets $\left(\varphi_{1}^{(\delta)}, \vec{x}_{1}^{(\delta)}\right)$ and $\left(\varphi_{2}^{(\delta)}, \vec{x}_{2}^{(\delta)}\right)$ in $V_{\delta, 1} \times \Gamma_{\delta}$ s.t.

$$
\left\|\Pi_{\delta}\left(\varphi_{1}^{(\delta)}, \vec{x}_{1}^{(\delta)}\right)-\Pi_{\delta}\left(\varphi_{2}^{(\delta)}, \vec{x}_{2}^{(\delta)}\right)\right\|>\varepsilon
$$

for any $\delta>0$. This inequality, however, contradicts the assumption that the norms of the maps $\Pi_{\delta}$ tend to zero with $\delta \rightarrow \infty$.

With the help of the above fact we restate Condition $C_{b}$ (b) as follows: For any $E \geq 0$ there exists such natural number $N_{0}(E)$ that for any $N>N_{0}(E)$ there holds

$$
\lim _{\delta \rightarrow \infty}\left\|\Pi_{E, N, \delta}^{\mathrm{c}}\right\|=0 .
$$

We rewrite the above relation more explicitly

$$
\lim _{\delta \rightarrow \infty} \sup _{\substack{A_{i} \in \mathfrak{A}_{\mathrm{c}}(\mathcal{O})_{1} \\ i \in\{1, \ldots, N\} \\ \underline{\underline{x}} \in \Gamma_{N, \delta}}}\left\|P_{E} A_{1}\left(\vec{x}_{1}\right) \ldots A_{N}\left(\vec{x}_{N}\right) P_{E}\right\|=0
$$

and use it to prove the following lemma which is the main technical result of this section.

Lemma 3.3.3. Suppose that Condition $C_{\mathrm{b}}$ holds. Then, for any $E \geq 0$, double cone $\mathcal{O}$, and a sequence $\{\delta(n)\}_{1}^{\infty}$ s.t. $\delta(n) \underset{n \rightarrow \infty}{\longrightarrow} \infty$, the following assertions hold true:

(a) For any family of points $\left\{\vec{x}_{i}^{(n)}\right\}_{1}^{n} \in \Gamma_{n, \delta(n)}$ there holds

$$
\lim _{n \rightarrow \infty} \sup _{\substack{\varphi \in \mathcal{T}_{E, 1} \\ A \in \mathfrak{A}_{c}(\mathcal{O})_{1}}}\left|\frac{1}{n} \sum_{i=1}^{n} \varphi\left(A\left(\vec{x}_{i}^{(n)}\right)\right)\right|=0 .
$$


(b) For any unit vector $\hat{e} \in \mathbb{R}^{s}$, sequence $\left\{\lambda^{(n)}\right\}_{1}^{\infty} \in \mathbb{R}$ and a family of points $\left\{\vec{x}_{i}^{(n)}\right\}_{1}^{n}$, s.t. $\left\{\vec{x}_{i}^{(n)}\right\}_{1}^{n} \cup\left\{\vec{x}_{i}^{(n)}+\lambda^{(n)} \hat{e}\right\}_{1}^{n} \in \Gamma_{2 n, \delta(n)}$, there holds

$$
\lim _{n \rightarrow \infty} \sup _{\substack{\varphi \in \mathcal{T}_{E, 1} \\ A, B \in \mathfrak{A}_{c}(\mathcal{O})_{1}}}\left|\frac{1}{n} \sum_{i=1}^{n} \varphi\left(A\left(\vec{x}_{i}^{(n)}\right) B\left(\vec{x}_{i}^{(n)}+\lambda^{(n)} \hat{e}\right)\right)\right|=0 .
$$

Proof. In view of decomposition (1.6.2) we can, without loss of generality, substitute $\mathcal{T}_{E, 1}^{+}$for $\mathcal{T}_{E, 1}$ in relations (3.3.11) and (3.3.12). Similarly, we can replace $\mathfrak{A}_{\mathrm{c}}(\mathcal{O})_{1}$ with the subset of its self-adjoint elements.

We choose some positive functional $\varphi \in \mathcal{T}_{E, 1}^{+}$, pick $m \in \mathbb{N}$ s.t. $N=2^{m}$ is sufficiently large to ensure that (3.3.10) holds. To prove (a), we define the operators $Q_{n}=\frac{1}{n} \sum_{i=1}^{n} A\left(\vec{x}_{i}^{(n)}\right), n \in \mathbb{N}$, where $A \in \mathfrak{A}_{\mathrm{c}}(\mathcal{O})$ is self-adjoint, assume that $n \geq N$ and compute

$$
\begin{aligned}
\left|\varphi\left(Q_{n}\right)\right|^{N} & \leq \varphi\left(Q_{n}^{N}\right)=\frac{1}{n^{N}} \sum_{i_{1}, \ldots, i_{N}} \varphi\left(A\left(\vec{x}_{i_{1}}^{(n)}\right) \ldots A\left(\vec{x}_{i_{N}}^{(n)}\right)\right) \\
& =\frac{1}{n^{N}} \sum_{\substack{i_{1}, \ldots, i_{N} \\
\forall k \neq l}} \varphi\left(A\left(\vec{x}_{i_{1}}^{(n)}\right) \ldots A\left(\vec{x}_{i_{N}}^{(n)}\right)\right) \\
& +\frac{1}{n^{N}} \sum_{\substack{i_{1}, \ldots, i_{l} \\
\exists_{k \neq l} \text { s.t. } i_{k}=i_{l}}} \varphi\left(A\left(\vec{x}_{i_{1}}^{(n)}\right) \ldots A\left(\vec{x}_{i_{N}}^{(n)}\right)\right) \\
& \leq \frac{1}{n^{N}} \sum_{\substack{i_{1}, \ldots, i_{N} \\
\forall_{k \neq i} \neq i_{k} \neq i_{l}}}\left\|P_{E} A\left(\vec{x}_{i_{1}}^{(n)}\right) \ldots A\left(\vec{x}_{i_{N}}^{(n)}\right) P_{E}\right\|+\frac{1}{n^{N}} \sum_{\substack{i_{1}, \ldots, i_{N} \\
i_{k \neq l} \text { s.t. } i_{k}=i_{l}}}\|A\|^{N} .
\end{aligned}
$$

In the first step above we applied the Cauchy-Schwarz inequality and in the third step we extracted from the resulting sum the terms in which all the operators are mutually spacelike separated. Clearly, there are $\left(\begin{array}{c}n \\ N\end{array}\right) N ! \leq n^{N}$ such terms. Therefore, the remainder (the last sum above) consists of

$$
n^{N}-\left(\begin{array}{c}
n \\
N
\end{array}\right) N ! \leq c_{N} n^{N-1}
$$

terms, where $c_{N}$ is some constant independent of $n$. There follows the estimate

$$
\left|\varphi\left(Q_{n}\right)\right|^{N} \leq \sup _{\substack{A \in \mathfrak{A}_{c}(\mathcal{O})_{1} \\\left(\vec{x}_{1}, \ldots, \vec{x}_{N}\right) \in \Gamma_{N, \delta(n)}}}\left\|P_{E} A\left(\vec{x}_{1}\right) \ldots A\left(\vec{x}_{N}\right) P_{E}\right\|+\frac{c_{N}}{n}\|A\|^{N}
$$

whose r.h.s. tends to zero with $n \rightarrow \infty$ by (3.3.10), uniformly in $\varphi \in \mathcal{T}_{E, 1}^{+}$, what concludes the proof of (3.3.11).

In order to prove (b), we proceed similarly: Let $\hat{Q}_{n}=\frac{1}{n} \sum_{i=1}^{n} A\left(\vec{x}_{i}^{(n)}\right) B\left(\vec{x}_{i}^{(n)}+\lambda^{(n)} \hat{e}\right)$, 
where $A, B \in \mathfrak{A}_{\mathrm{c}}(\mathcal{O})$ are self-adjoint. Then, for $n \geq N$, we obtain

$$
\begin{aligned}
& \left|\varphi\left(\hat{Q}_{n}\right)\right|^{N} \leq \varphi\left(\hat{Q}_{n}^{N}\right) \\
= & \frac{1}{n^{N}} \sum_{i_{1}, \ldots, i_{N}} \varphi\left(A\left(\vec{x}_{i_{1}}^{(n)}\right) B\left(\vec{x}_{i_{1}}^{(n)}+\lambda^{(n)} \hat{e}\right) \ldots A\left(\vec{x}_{i_{N}}^{(n)}\right) B\left(\vec{x}_{i_{N}}^{(n)}+\lambda^{(n)} \hat{e}\right)\right) \\
= & \frac{1}{n^{N}} \sum_{\substack{i_{1}, \ldots, i_{N} \\
\forall k \neq l i_{k} \neq i_{l}}} \varphi\left(A\left(\vec{x}_{i_{1}}^{(n)}\right) B\left(\vec{x}_{i_{1}}^{(n)}+\lambda^{(n)} \hat{e}\right) \ldots A\left(\vec{x}_{i_{N}}^{(n)}\right) B\left(\vec{x}_{i_{N}}^{(n)}+\lambda^{(n)} \hat{e}\right)\right) \\
+ & \frac{1}{n^{N}} \sum_{\substack{i_{1}, \ldots, i_{N} \\
\exists_{k \neq l s} s . t i_{k}=i_{l}}} \varphi\left(A\left(\vec{x}_{i_{1}}^{(n)}\right) B\left(\vec{x}_{i_{1}}^{(n)}+\lambda^{(n)} \hat{e}\right) \ldots A\left(\vec{x}_{i_{N}}^{(n)}\right) B\left(\vec{x}_{i_{N}}^{(n)}+\lambda^{(n)} \hat{e}\right)\right) \\
\leq & \frac{1}{n^{N}} \sum_{\substack{i_{1}, \ldots, i_{N} \\
\forall k \neq l i i_{k} \neq i_{l}}}\left\|P_{E} A\left(\vec{x}_{i_{1}}^{(n)}\right) B\left(\vec{x}_{i_{1}}^{(n)}+\lambda^{(n)} \hat{e}\right) \ldots A\left(\vec{x}_{i_{N}}^{(n)}\right) B\left(\vec{x}_{i_{N}}^{(n)}+\lambda^{(n)} \hat{e}\right) P_{E}\right\| \\
+ & \frac{1}{n^{N}} \sum_{\substack{i_{1}, \ldots, i_{N} \\
\exists_{k \neq l} s . t i_{k}=i_{l}}}(\|A\|\|B\|)^{N} .
\end{aligned}
$$

By the same reasoning as in case (a), we obtain the estimate

$$
\begin{aligned}
\left|\varphi\left(\hat{Q}_{n}\right)\right|^{N} \leq & \sup _{\substack{A \in \mathfrak{A}_{c}(\mathcal{O})_{1} \\
\left(\vec{x}_{1}, \ldots, \vec{x}_{2 N}\right) \in \Gamma_{2 N, \delta(n)}}}\left\|P_{E} A\left(\vec{x}_{1}\right) B\left(\vec{x}_{2}\right) \ldots A\left(\vec{x}_{2 N-1}\right) B\left(\vec{x}_{2 N}\right) P_{E}\right\| \\
+ & \frac{c_{N}}{n}(\|A\|\|B\|)^{N} .
\end{aligned}
$$

By taking the limit $n \rightarrow \infty$ we conclude the proof of (3.3.12).

Now we are ready to prove that the vacuum state $\omega_{0}$ is pure and unique in the energetically connected component of the state space.

Theorem 3.3.4. Suppose that Condition $C_{b}$ is satisfied. Then there hold the following assertions:

(a) Let $\omega \in \mathfrak{A}^{*}$ be a state in the weak $k^{*}$ closure of $\mathcal{T}_{E, 1}$ for some $E \geq 0$ which is invariant under translations in some spacelike ray. Then $\omega=\omega_{0}$. In particular, there holds Condition $V$ stated in Section 2.2.

(b) $\omega_{0}$ is a pure state.

Proof. (a) By Theorem 3.1.3 $\omega$ is a vacuum state, in particular it is translationally invariant. Let $\left\{\varphi_{\beta}\right\}_{\beta \in I}$ be a net of functionals from $\mathcal{T}_{E, 1}$ approximating $\omega$ in the weak* topology and let $A \in \mathfrak{A}_{\mathrm{c}}(\mathcal{O})$ i.e. $\omega_{0}(A)=0$. We choose families of points $\left\{\vec{x}_{i}\right\}_{1}^{n}$ in $\mathbb{R}^{s}$ s.t. $\left\{\vec{x}_{i}\right\}_{1}^{n} \in \Gamma_{n, \delta(n)}$ for some sequence $\{\delta(n)\}_{1}^{\infty}$ which diverges to infinity with $n \rightarrow \infty$. We note the following relation

$$
\begin{aligned}
|\omega(A)| & =\left|\frac{1}{n} \sum_{i=1}^{n} \omega\left(A\left(\vec{x}_{i}^{(n)}\right)\right)\right|=\lim _{\beta}\left|\frac{1}{n} \sum_{i=1}^{n} \varphi_{\beta}\left(A\left(\vec{x}_{i}^{(n)}\right)\right)\right| \\
& \leq \sup _{\varphi \in \mathcal{T}_{E, 1}}\left|\frac{1}{n} \sum_{i=1}^{n} \varphi\left(A\left(\vec{x}_{i}^{(n)}\right)\right)\right| \underset{n \rightarrow \infty}{\longrightarrow} 0
\end{aligned}
$$


where in the first step we made use of the fact that the state $\omega$ is invariant under translations in space and in the last step we made use of Lemma 3.3.3 (a). Since local algebras are norm dense in the global algebra $\mathfrak{A}$, we conclude that $\operatorname{ker} \omega_{0} \subset \operatorname{ker} \omega$ and therefore the two states are equal.

(b) In order to show the purity of $\omega_{0}$, it suffices to verify that for any $A, B \in \mathfrak{A}_{\mathrm{c}}(\mathcal{O})$, some unit vector $\hat{e} \in \mathbb{R}^{s}$ and some sequence of real numbers $\left\{\lambda^{(n)}\right\}_{1}^{\infty}$ s.t. $\lambda^{(n)} \underset{n \rightarrow \infty}{\longrightarrow} \infty$ there holds

$$
\lim _{n \rightarrow \infty} \omega_{0}\left(A B\left(\lambda^{(n)} \hat{e}\right)\right)=0 .
$$

To this end, we recall that the vacuum state $\omega_{0}$ is energetically accessible i.e. we can pick a net $\left\{\varphi_{\beta}\right\}_{\beta \in I}$ of functionals from $\mathcal{T}_{E, 1}$, approximating $\omega_{0}$ in the weak* topology. Next, we choose families of points $\left\{\vec{x}_{i}^{(n)}\right\}_{1}^{n}$ as in part (b) of Lemma 3.3.3 and compute

$$
\begin{aligned}
\left|\omega_{0}\left(A B\left(\lambda^{(n)} \hat{e}\right)\right)\right| & =\left|\frac{1}{n} \sum_{i=1}^{n} \omega_{0}\left(A\left(\vec{x}_{i}^{(n)}\right) B\left(\vec{x}_{i}^{(n)}+\lambda^{(n)} \hat{e}\right)\right)\right| \\
& =\lim _{\beta}\left|\frac{1}{n} \sum_{i=1}^{n} \varphi_{\beta}\left(A\left(\vec{x}_{i}^{(n)}\right) B\left(\vec{x}_{i}^{(n)}+\lambda^{(n)} \hat{e}\right)\right)\right| \\
& \leq \sup _{\varphi \in \mathcal{T}_{E, 1}}\left|\frac{1}{n} \sum_{i=1}^{n} \varphi\left(A\left(\vec{x}_{i}^{(n)}\right) B\left(\vec{x}_{i}^{(n)}+\lambda^{(n)} \hat{e}\right)\right)\right| \underset{n \rightarrow \infty}{\longrightarrow} 0,
\end{aligned}
$$

what proves relation (3.3.19).

From part (a) of the above theorem and from Propositions 3.1.4 and 3.1.5 we readily obtain the convergence of states of bounded energy to the vacuum state $\omega_{0}$ under large spacelike or timelike translations.

Corollary 3.3.5. Let Condition $C_{\mathrm{b}}$ be satisfied. Then, for any state $\omega \in \mathcal{T}_{E}, E \geq 0$, and a spacelike unit vector $\hat{e} \in \mathbb{R}^{s+1}$, there holds

$$
\lim _{\lambda \rightarrow \infty} \omega_{\lambda \hat{e}}(A)=\omega_{0}(A) \text { for } A \in \mathfrak{A} .
$$

If, in addition, there holds Condition $R$, then the above relation is also true for any timelike unit vector $\hat{e}$.

To conclude this survey of applications of Condition $C_{\mathrm{b}}$, let us mention another physically meaningful procedure for preparation of vacuum states: This is to construct functionals with increasingly sharp values of energy and momentum and exploit the uncertainty principle. Let $P_{(p, r)}$ be the spectral projection corresponding to the ball of radius $r$ centered around some point $p$ in the energy-momentum spectrum and $\mathcal{T}_{(p, r)}=P_{(p, r)} \mathcal{T} P_{(p, r)}$. Then, in a theory satisfying Condition $C_{b}$, any sequence of states $\omega_{r} \in \mathcal{T}_{(p, r)}$ converges, uniformly on local algebras, to the vacuum state $\omega_{0}$ as $r \rightarrow 0$, since this is the only energetically accessible state which is completely dislocalized in spacetime. This fact is reflected in the following proposition:

Proposition 3.3.6. Suppose that Condition $C_{b}$ is satisfied. Then, for any $p \in \bar{V}_{+}$and double cone $\mathcal{O}$, there holds

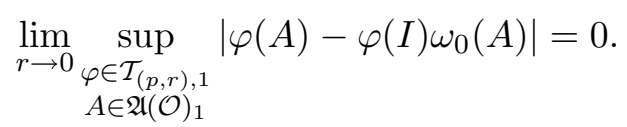


Proof. We pick $A \in B(\mathcal{H}), \varphi \in \mathcal{T}_{(p, r)}, \vec{x} \in \mathbb{R}^{s}$ and estimate the deviation of this functional from the translational invariance

$$
\begin{aligned}
\left|\varphi(A)-\alpha_{\vec{x}}^{*} \varphi(A)\right| & =\left|\varphi\left(P_{(p, r)} A P_{(p, r)}\right)-\varphi\left(P_{(p, r)} e^{i(\vec{P}-\vec{p}) \vec{x}} A e^{-i(\vec{P}-\vec{p}) \vec{x}} P_{(p, r)}\right)\right| \\
& =\mid \varphi\left(P_{(p, r)} e^{i(\vec{P}-\vec{p}) \vec{x}} A\left(1-e^{-i(\vec{P}-\vec{p}) \vec{x}}\right) P_{(p, r)}\right) \\
& +\varphi\left(P_{(p, r)}\left(1-e^{i(\vec{P}-\vec{p}) \vec{x}}\right) A P_{(p, r)}\right)|\leq 2\|\varphi\|\|A\|| \vec{x} \mid r
\end{aligned}
$$

where in the last step we applied the spectral theorem. Consequently, for any $\vec{x}_{1}, \ldots, \vec{x}_{n} \in$ $\mathbb{R}^{s}$ and $A \in \mathfrak{A}(\mathcal{O})$ there holds

$$
\begin{array}{r}
\left|\varphi(A)-\varphi(I) \omega_{0}(A)\right| \leq\left|\frac{1}{n} \sum_{k=1}^{n}\left[\varphi(A)-\varphi\left(A\left(\vec{x}_{k}\right)\right)\right]\right|+\left|\frac{1}{n} \sum_{k=1}^{n} \varphi\left(A\left(\vec{x}_{k}\right)-\omega_{0}(A) I\right)\right| \\
\leq\|A\| \sup _{k \in\{1, \ldots, N\}}\left\|\varphi-\alpha_{\vec{x}_{k}}^{*} \varphi\right\|+2\|A\| \sup _{B \in \mathfrak{A}_{c}(\mathcal{O})_{1}}\left|\frac{1}{n} \sum_{k=1}^{n} \varphi\left(B\left(\vec{x}_{k}\right)\right)\right| .
\end{array}
$$

Applying to the first term on the r.h.s. estimate (3.3.23) and to the second term Lemma 3.3 .3 (a) we obtain the statement of the proposition.

\subsection{Condition $C_{\sharp}$ : Additivity of Energy}

In the previous section we introduced the phase space condition $C_{\mathrm{b}}$, inspired by the physically expected behavior of coincidence arrangements of local detectors. We showed that this condition implies the uniqueness and the purity of the energetically accessible vacuum state as well as various approximation procedures for this state. However, its status in massless theories is not clear. Since such theories play an important role in our study of spectral theory of automorphism groups in Chapter 2] we think it is worthwhile to fill this gap. For this purpose we introduce a different phase space condition $C_{\natural}$ stated below. We note that in contrast to Condition $C_{\mathrm{b}}$ the vacuum state does not enter into the formulation of the present condition. Thus Condition $C_{\sharp}$ is a property of the local net and not of a particular vacuum state, what is certainly an advantage. This criterion is motivated by a heuristic argument based on the additivity of energy over isolated subregions and it is shown that it has all the consequences mentioned in Section 3.3 (except for the purity of the vacuum). In Section 3.5 and Appendix E we verify that this condition holds in massive and massless scalar free field theory. In Chapter 4 we discuss its generalizations which may be useful for the problem of convergence of the asymptotic functional approximants.

The concept of additivity of energy does not have an unambiguous meaning in the general framework of local relativistic quantum field theory and we rely here on the following formulation: We introduce the family of maps $\Sigma_{E, N, \delta} \in \mathcal{L}\left(\mathcal{T}_{E} \times \Gamma_{N, \delta}, \mathfrak{A}(\mathcal{O})^{*} \otimes \mathbb{C}_{\text {sup }}^{N}\right)$, given by

$$
\Sigma_{E, N, \delta}(\varphi, \underline{\vec{x}})=\left(\Pi_{E}\left(\varphi_{\vec{x}_{1}}\right), \ldots, \Pi_{E}\left(\varphi_{\vec{x}_{N}}\right)\right),
$$

where $\mathbb{C}_{\text {sup }}^{N}$ denotes the space $\mathbb{C}^{N}$ with the norm $\|\underline{z}\|=\sup _{k \in\{1, \ldots, N\}}\left|z_{k}\right|$. We note that $\mathfrak{A}(\mathcal{O})^{*} \otimes \mathbb{C}_{\text {sup }}^{N}$, equipped with the norm,

$$
\left\|\left(\varphi_{1}, \ldots, \varphi_{N}\right)\right\|=\sup _{k \in\{1, \ldots, N\}}\left\|\varphi_{k}\right\|
$$


is a Banach space. It is clear that the map $\Sigma_{E, N, \delta}$ is compact in theories satisfying Condition $C_{\sharp}$. We claim that a mild (polynomial) growth of the $\varepsilon$-contents $\mathcal{N}(\varepsilon)_{E, N, \delta}$ of these maps with $N$, (when $\delta$ tends to infinity), is a signature of the additivity of energy over isolated subregions. In order to justify this formulation we provide a heuristic argument:

The physical meaning of the maps $\Sigma_{E, N, \delta}$ is most easily elucidated if we consider their restrictions to $S_{E} \times \Gamma_{N, \delta}$. Thus we are interested in the $\varepsilon$-contents $\widetilde{\mathcal{N}}(\varepsilon)_{E, N, \delta}$ of the set $\mathbb{1}$

$$
S_{E, N, \delta}(\mathcal{O})=\left\{\left(\Pi_{E}\left(\omega_{\vec{x}_{1}}\right), \ldots, \Pi_{E}\left(\omega_{\vec{x}_{N}}\right)\right) \in \mathfrak{A}(\mathcal{O})^{*} \otimes \mathbb{C}_{\text {sup }}^{N} \mid \omega \in S_{E}, \underline{\vec{x}} \in \Gamma_{N, \delta}\right\} .
$$

Given a state $\omega \in S_{E}$, we denote by $E_{k}$ the 'local energy content' of the restricted state $\left.\omega\right|_{\mathfrak{A}\left(\mathcal{O}+x_{k}\right)}$. The additivity of energy should then imply that $E_{1}+\cdots+E_{N} \leq E$ for large spacelike distance $\delta$ between the regions $\mathcal{O}+\vec{x}_{1}, \ldots, \mathcal{O}+\vec{x}_{N}$, where $\underline{\vec{x}} \in \Gamma_{N, \delta}$. This suggests that to calculate $\tilde{\mathcal{N}}(\varepsilon)_{E, N, \delta}$ one should count all the families of states $\left(\omega_{1}, \ldots, \omega_{N}\right), \omega_{k} \in$ $S_{E_{k}}, E_{1}+\cdots+E_{N} \leq E$, which can be distinguished, up to accuracy $\varepsilon$, by measurements in $\mathcal{O}+\vec{x}_{1}, \ldots, \mathcal{O}+\vec{x}_{N}$. Relying on this heuristic reasoning we write

$$
\begin{array}{r}
\tilde{\mathcal{N}}(\varepsilon)_{E, N, \delta}=\#\left\{\left(n_{1}, \ldots, n_{N}\right) \in \mathbb{N}^{N} \mid n_{1} \leq \tilde{\mathcal{N}}(\varepsilon)_{E_{1}}, \ldots, n_{N} \leq \tilde{\mathcal{N}}(\varepsilon)_{E_{N}},\right. \\
\left.\quad \text { for some } E_{1}, \ldots, E_{N} \geq 0 \text { s.t. } E_{1}+\cdots+E_{N} \leq E\right\},
\end{array}
$$

where we made use of the fact that the number of states from $S_{E_{k}}$ which can be discriminated, up to $\varepsilon$, by observables localized in the region $\mathcal{O}+x_{k}$ is equal to the $\varepsilon$-content $\tilde{\mathcal{N}}(\varepsilon)_{E_{k}}$ of the set

$$
S_{E_{k}}\left(\mathcal{O}+\vec{x}_{k}\right)=\left\{\left.\omega\right|_{\mathfrak{A}\left(\mathcal{O}+\vec{x}_{k}\right)} \mid \omega \in S_{E_{k}}\right\} .
$$

Anticipating that $\tilde{\mathcal{N}}(\varepsilon)_{E_{k}}$ tends to one for small $E_{k}$, we may assume that

$$
\tilde{\mathcal{N}}(\varepsilon)_{E_{k}} \leq 1+c_{0}(\varepsilon, E) E_{k}
$$

for $E_{k} \leq E$. This bound obviously holds in theories with the lower mass gap, satisfying Condition $C_{\sharp}$, where $S_{E}$ contains at most the vacuum state $(\Omega \mid \cdot \Omega)$ for sufficiently small $E$. (If the vacuum vector $\Omega$ exists, it must be unique (up to a phase) by the irreducibility assumption from Section [1.6] See e.g. Theorem 4.6 of [Ar]). We also expect that estimate (3.4.6) holds in massless theories, where de Broglie wavelengths of states from $S_{E_{k}}\left(\mathcal{O}+\vec{x}_{k}\right)$ are much larger than the extent of the region $\mathcal{O}+\vec{x}_{k}$ if $E_{k}$ is sufficiently small. Thus the states should be indistinguishable by measurements in this region, up to the experimental accuracy $\varepsilon$. (The existence of massless theories satisfying the bound (3.4.6) is indicated in the last part of this section). From the heuristic formula (3.4.4) and the bound (3.4.6) we obtain the estimate which grows only polynomially with $N$

$$
\begin{aligned}
\tilde{\mathcal{N}}(\varepsilon)_{E, N, \delta} \leq \#\left\{\left(n_{1}, \ldots, n_{N}\right) \in \mathbb{N}^{N} \mid n_{1}+\cdots+n_{N}\right. & \left.\leq N+c_{0}(\varepsilon, E) E\right\} \\
& \leq(N+1)^{c_{0}(\varepsilon, E) E}
\end{aligned}
$$

where the last inequality can be verified by induction in $N$. Omitting the key condition $E_{1}+\cdots+E_{N} \leq E$ in (3.4.4) and setting $E_{k}=E$ instead, one would arrive at an exponential growth of $\widetilde{\mathcal{N}}(\varepsilon)_{E, N, \delta}$ as a function of $N$. Thus the moderate (polynomial) increase of this quantity with regard to $N$ is in fact a clear-cut signature of the additivity of energy over isolated subsystems. We encode this fact into the following strengthened variant of Condition $C_{\sharp}$.

\footnotetext{
${ }^{1}$ The $\varepsilon$-content $\widetilde{\mathcal{N}}(\varepsilon)$ of some set $S$ in a Banach space is the maximal number of elements $\omega_{1}, \ldots, \omega_{\mathcal{N}(\varepsilon)} \in$ $S$ s.t. $\left\|\omega_{i}-\omega_{j}\right\|>\varepsilon$ for $i \neq j$.
} 


\section{Condition $C_{\natural}$ :}

(a) The maps $\Sigma_{E, N, \delta}$ are compact for any $E \geq 0, N \in \mathbb{N}, \delta>0$ and double cone $\mathcal{O}$.

(b) The $\varepsilon$-contents $\mathcal{N}(\varepsilon)_{E, N, \delta}$ of the maps $\Sigma_{E, N, \delta}$ satisfy, for any $\varepsilon>0$,

$$
\lim _{\delta \rightarrow \infty} \mathcal{N}(\varepsilon)_{E, N, \delta} \leq(N+1)^{c(E, \varepsilon)},
$$

for some constant $c(E, \varepsilon)$ independent of $N$.

The remaining part of this section is devoted to the proof that Condition $C_{\natural}$ has all the consequences, pertaining to the vacuum structure, which were discussed in Section 3.3 (apart from the purity of the vacuum). For this purpose it suffices to show that the present condition implies relation (3.3.11) for some specific sequence $\left\{\delta_{n}\right\}_{n \in \mathbb{N}}$ and some energetically accessible vacuum state $\omega_{0}$. This goal will be accomplished in the two lemmas below. Since no distinguished vacuum state enters into Condition $C_{\mathfrak{\natural}}$, we have to prepare such a state first: We fix a unit vector $\hat{e}$ in a space direction and obtain from Proposition 3.1 .4 a net of real numbers $\left\{\lambda_{\beta}\right\}_{\beta \in \mathbb{I}}$ s.t. $\lambda_{\beta} \rightarrow \infty$ and a vacuum state $\omega_{0}$ s.t. for any $A \in \mathfrak{A}$

$$
\mathrm{w}^{*}-\lim _{\beta} A\left(\lambda_{\beta} \hat{e}\right)=\omega_{0}(A) I \text {. }
$$

We will call the triple $\left\{\hat{e},\left\{\lambda_{\beta}\right\}_{\beta \in \mathbb{I}}, \omega_{0}\right\}$ the spacelike asymptotic vacuum state.

In the subsequent discussion we keep $E \geq 0$ and a double cone $\mathcal{O}$ fixed. Moreover, for any $\omega \in S_{E}$ and $\underline{\vec{x}} \in \Gamma_{N, \delta}$ we denote by $\omega^{\underline{\vec{x}}}$ the element of $S_{E, N, \delta}(\mathcal{O})$ given by

$$
\omega^{\underline{\vec{x}}}(A)=\left(\omega\left(A\left(\vec{x}_{1}\right)\right), \ldots, \omega\left(A\left(\vec{x}_{N}\right)\right)\right), \quad A \in \mathfrak{A}(\mathcal{O}) .
$$

Furthermore, $\bar{S}_{E, N, \delta}(\mathcal{O})$ denotes the closure of $S_{E, N, \delta}(\mathcal{O})$ in $\mathfrak{A}(\mathcal{O})^{*} \otimes \mathbb{C}_{\text {sup }}^{N}$ in the topology given by the norm (3.4.2). The following simple lemma summarizes the essential properties of the sets $\bar{S}_{E, N, \delta}(\mathcal{O})$.

Lemma 3.4.1. Assume that Condition $C_{\sharp}$ is satisfied. Let $\hat{\omega}=\left(\hat{\omega}_{1}, \ldots, \hat{\omega}_{N}\right) \in \bar{S}_{E, N, \delta}(\mathcal{O})$, let $\left\{\hat{e},\left\{\lambda_{\beta}\right\}_{\beta \in \mathbb{I}}, \omega_{0}\right\}$ be a spacelike asymptotic vacuum state and $\mathcal{P}_{N}$ the group of permutations of an $N$-element set. Then:

(a) $\hat{\omega}_{\pi}:=\left(\hat{\omega}_{\pi(1)}, \ldots, \hat{\omega}_{\pi(N)}\right) \in \bar{S}_{E, N, \delta}(\mathcal{O})$, for any $\pi \in \mathcal{P}_{N}$.

(b) $\hat{\omega}^{\prime}:=\left(\hat{\omega}_{1}, \ldots, \hat{\omega}_{N-1}\right) \in \bar{S}_{E, N-1, \delta}(\mathcal{O})$.

(c) $\hat{\omega}^{\prime \prime}:=(\hat{\omega}_{1}, \ldots, \hat{\omega}_{N}, \underbrace{\omega_{0}, \ldots, \omega_{0}}_{L}) \in \bar{S}_{E, N+L, \delta}(\mathcal{O})$.

Proof. To prove part (a), we first define the action of the group of permutations on the sets $\Gamma_{N, \delta}$. Given $\underline{\vec{x}}=\left(\vec{x}_{1}, \ldots, \vec{x}_{N}\right) \in \Gamma_{N, \delta}$ and $\pi \in \mathcal{P}_{N}$, we set $\underline{\vec{x}}_{\pi}=\left(\vec{x}_{\pi(1)}, \ldots, \vec{x}_{\pi(N)}\right)$ which is again an element of $\Gamma_{N, \delta}$. This induces an action of permutations on the sets $S_{E, N, \delta}(\mathcal{O})$ in the obvious manner: Given $\omega^{\underline{\vec{x}}} \in S_{E, N, \delta}(\mathcal{O})$ we define $\left(\omega^{\overrightarrow{\underline{x}}}\right)_{\pi}=\omega^{\underline{\vec{x}}_{\pi}}$. Consequently, all the sets $S_{E, N, \delta}(\mathcal{O}), \delta>0$ are invariant under the permutations of the entries of their elements. This property carries over to their closures: In fact, given $\hat{\omega} \in \bar{S}_{E, N, \delta}(\mathcal{O})$, there exists for every $\varepsilon>0$ some $\omega^{\underline{\vec{x}}} \in S_{E, N, \delta}(\mathcal{O})$ s.t. $\left\|\omega^{\underline{\vec{x}}}-\hat{\omega}\right\| \leq \varepsilon$. Then $\left\|\omega^{\underline{\underline{x}}_{\pi}}-\hat{\omega}_{\pi}\right\| \leq \varepsilon$. 
Part (b) of the lemma follows directly from the definition of the sets $S_{E, N, \delta}(\mathcal{O})$ and their closures. In order to prove part (c) we pick again $\omega^{\underline{\vec{x}}} \in S_{E, N, \delta}(\mathcal{O})$ s.t. $\left\|\omega^{\underline{\vec{x}}}-\hat{\omega}\right\| \leq \varepsilon$. According to Lemma 3.1.2 there exists a subsequence $\left\{\lambda_{n}\right\}_{n \in \mathbb{N}}$ of $\left\{\lambda_{\beta}\right\}_{\beta \in \mathbb{I}}$ s.t.

$$
\lim _{n \rightarrow \infty} \omega_{\lambda_{n} \hat{e}}(A)=\omega_{0}(A), \quad A \in \mathfrak{A} .
$$

We choose its subsequences $\lambda_{n}^{(1)}, \ldots, \lambda_{n}^{(L)}$ s.t. for $k \neq l$ there holds $\left|\lambda_{n}^{(k)}-\lambda_{n}^{(l)}\right| \rightarrow \infty$ when $n \rightarrow \infty$. Consequently,

$$
\hat{\omega}_{n}^{\prime \prime}:=\left(\omega_{\vec{x}_{1}}, \ldots, \omega_{\vec{x}_{N}}, \omega_{\vec{x}_{1}+\lambda_{n}^{(1)} \hat{e}}, \ldots, \omega_{\vec{x}_{1}+\lambda_{n}^{(L)}}\right) \in S_{E, N+L, \delta}
$$

for sufficiently large $n$, where $\hat{e}$ is the unit vector in a space direction which entered into the construction of the state $\omega_{0}$. It follows immediately from (3.4.11) and Condition $C_{\sharp}$ that

$$
\lim _{n \rightarrow \infty}\left\|\omega_{\vec{x}_{1}+\lambda_{n}^{(k)} \hat{e}}-\omega_{0}\right\|_{\mathfrak{A}(\mathcal{O})}=0
$$

for $k \in\{1, \ldots, L\}$. Consequently, $\lim _{n \rightarrow \infty}\left\|\hat{\omega}_{n}^{\prime \prime}-\hat{\omega}^{\prime \prime}\right\| \leq \varepsilon$, what concludes the proof.

The next lemma demonstrates that a state of bounded energy can deviate only locally from a vacuum state.

Lemma 3.4.2. Suppose that Condition $C_{\boxminus}$ holds. Let $\omega_{0}$ be the vacuum state which appears in Lemma 3.4.1. Then there exists a sequence of positive numbers $\left\{\delta_{N}\right\}_{N \in \mathbb{N}}$ s.t. $\delta_{N} \nearrow \infty$ and

$$
\sup _{\hat{\omega} \in \bar{S}_{E, N, \delta_{N}}(\mathcal{O})} \frac{1}{N} \sum_{k=1}^{N}\left\|\hat{\omega}_{k}-\omega_{0}\right\|_{\mathfrak{A}(\mathcal{O})} \rightarrow 0 \text { for } N \rightarrow \infty .
$$

Proof. First, making use of Condition $C_{\natural}$ and the diagonal trick, we can find a sequence $\delta_{N} \nearrow \infty$ s.t. for any $\varepsilon>0$ the $\varepsilon$-contents $\widetilde{\mathcal{N}}(\varepsilon)_{E, N, \delta_{N}}$ of the sets $S_{E, N, \delta_{N}}(\mathcal{O})$ satisfy

$$
\widetilde{\mathcal{N}}(\varepsilon)_{E, N, \delta_{N}} \leq 2(N+1)^{c(\varepsilon, E)},
$$

if $N$ is sufficiently large. Next, we fix some $\varepsilon>0,0<q<1$ and show that for any $\hat{\omega}=\left(\hat{\omega}_{1}, \ldots, \hat{\omega}_{N}\right) \in \bar{S}_{E, N, \delta_{N}}(\mathcal{O})$, the inequality

$$
\left\|\hat{\omega}_{k}-\omega_{0}\right\|_{\mathfrak{A}(\mathcal{O})}>\varepsilon
$$

holds for less than $\left[N^{q}\right]$ entries if $N$ is sufficiently large. In fact, suppose the opposite is true i.e. that for any $N_{0} \in \mathbb{N}$ there exists $N>N_{0}$ and an element $\hat{\omega} \in \bar{S}_{E, N, \delta_{N}}(\mathcal{O})$ s.t. the bound (3.4.16) holds for $\left[N^{q}\right]$ entries or more. Making use of Lemma 3.4.1 (a) we can assume that (3.4.16) is satisfied for $k \in\left\{1, \ldots,\left[N^{q}\right]\right\}$ and proceed to the element

$$
\hat{\omega}^{\prime \prime}:=(\hat{\omega}_{1}, \ldots, \hat{\omega}_{\left[N^{q}\right]}, \underbrace{\omega_{0}, \ldots, \omega_{0}}_{N-\left[N^{q}\right]}) \in \bar{S}_{E, N, \delta_{N}}(\mathcal{O}) .
$$

By permuting the entries of the above expression, we obtain a family of elements $\hat{\omega}_{\pi}^{\prime \prime} \in$ $\bar{S}_{E, N, \delta_{N}}(\mathcal{O})$ s.t.

$$
\left\|\hat{\omega}_{\pi_{1}}^{\prime \prime}-\hat{\omega}_{\pi_{2}}^{\prime \prime}\right\|>\varepsilon
$$


at least for $\pi_{1}, \pi_{2} \in \tilde{\mathcal{P}}_{N}:=\mathcal{P}_{N} /\left(\mathcal{P}_{\left[N^{q}\right]} \times \mathcal{P}_{N-\left[N^{q}\right]}\right), \pi_{1} \neq \pi_{2}$. The cardinality of $\tilde{\mathcal{P}}_{N}$ satisfies the bound

$$
\# \tilde{\mathcal{P}}_{N}=\frac{N !}{\left[N^{q}\right] !\left(N-\left[N^{q}\right]\right) !} \geq\left(\frac{N-\left[N^{q}\right]+1}{\left[N^{q}\right]}\right)^{\left[N^{q}\right]} \geq 2^{N^{q}-1},
$$

where the last inequality holds for sufficiently large $N$. It follows from formulas (3.4.18), (3.4.19) that the $\varepsilon$-contents of the sets $\bar{S}_{E, N, \delta_{N}}(\mathcal{O})$ grow with $N$ faster than any polynomial. Since the $\varepsilon$-content of a set and its closure coincide, we arrive at a contradiction with relation (3.4.15).

With the above information at hand it is easy to estimate the mean, appearing in the statement of the lemma. In fact, for sufficiently large $N$ we obtain

$$
\sup _{\hat{\omega} \in \bar{S}_{E, N, \delta_{N}}(\mathcal{O})} \frac{1}{N} \sum_{k=1}^{N}\left\|\hat{\omega}_{k}-\omega_{0}\right\|_{\mathfrak{A}(\mathcal{O})} \leq 2 \frac{N^{q}}{N}+\frac{N-\left[N^{q}\right]}{N} \varepsilon \leq \frac{2}{N^{1-q}}+\varepsilon .
$$

Since $\varepsilon>0$ is arbitrary, the desired result follows.

It is an immediate consequence of the above lemma and of decomposition (1.6.2) that for any $\underline{\vec{x}}^{(N)}=\left(x_{1}^{(N)}, \ldots, x_{N}^{(N)}\right) \in \Gamma_{N, \delta_{N}}$ there holds

$$
\lim _{N \rightarrow \infty} \sup _{\substack{\varphi \in \mathcal{T}_{E, 1} \\ A \in \mathfrak{A}(\mathcal{O})_{1}}} \frac{1}{N} \sum_{k=1}^{N}\left|\varphi\left(A\left(x_{k}^{(N)}\right)\right)-\varphi(I) \omega_{0}(A)\right|=0 .
$$

This statement coincides with relation (3.3.11) from Lemma 3.3.3 (for the special sequence $\left\{\delta_{N}\right\}_{N \in \mathbb{N}}$ introduced in Lemma 3.4.2). Since Condition $C_{\mathrm{b}}$ was used only via this relation in the proofs of Theorem 3.3.4 (a), Corollary 3.3.5 and Proposition 3.3.6 these results still hold after replacing Condition $C_{\mathrm{b}}$ with Condition $C_{\sharp}$ in their assumptions. Thus we arrive at the following theorem.

Theorem 3.4.3. Suppose that Condition $C_{\boldsymbol{G}}$ is satisfied and let $\omega_{0}$ be any vacuum state in the weak* closure of $\mathcal{T}_{E, 1}$ for some $E \geq 0$. Then, for any $E \geq 0$, there hold the following assertions:

(a) Let $\omega \in \mathfrak{A}^{*}$ be a state in the weak $k^{*}$ closure of $\mathcal{T}_{E, 1}$ which is invariant under translations in some spacelike ray. Then $\omega=\omega_{0}$. In particular there holds Condition $V$ stated in Section 2.2.

(b) For any spacelike unit vector $\hat{e} \in \mathbb{R}^{s+1}$ and $\omega \in S_{E}$ there holds

$$
\lim _{\lambda \rightarrow \infty} \omega_{\lambda \hat{e}}(A)=\omega_{0}(A) \text { for } A \in \mathfrak{A} .
$$

If, in addition, Condition R, stated in Section 3.1, is satisfied, then the above relation is also true for any timelike unit vector $\hat{e}$.

(c) For any $p \in \bar{V}_{+}$and double cone $\mathcal{O}$, there holds

$$
\lim _{r \rightarrow 0} \sup _{\substack{\varphi \in \mathcal{T}_{(p, r), 1} \\ A \in \mathfrak{A}(\mathcal{O})_{1}}}\left|\varphi(A)-\varphi(I) \omega_{0}(A)\right|=0
$$


Part (c) of this theorem allows us to show that estimate (3.4.6), which we used in our heuristic discussion, holds in all theories complying with Condition $C_{\mathfrak{日}}$. Since the $\varepsilon$ contents $\tilde{\mathcal{N}}(\varepsilon)_{E}$ of the sets $S_{E}(\mathcal{O})$, given by (3.4.5), take only integer values, it suffices to show that

$$
\lim _{E \downarrow 0} \widetilde{\mathcal{N}}(\varepsilon)_{E}=1
$$

for any $\varepsilon>0$. If this relation did not hold, then, by definition of the $\varepsilon$-content, we could find nets $\left\{\omega_{1}^{E}\right\}_{E>0},\left\{\omega_{2}^{E}\right\}_{E>0}$ s.t. $\left\|\omega_{1}^{E}-\omega_{2}^{E}\right\|_{\mathfrak{A}(\mathcal{O})}>\varepsilon$ for any $E>0$. But choosing $p=0$ in Theorem 3.4 .3 (c), we arrive at a contradiction:

$$
\left\|\omega_{1}^{E}-\omega_{2}^{E}\right\|_{\mathfrak{A}(\mathcal{O})} \leq 2 \sup _{\omega \in S_{E}}\left\|\omega-\omega_{0}\right\|_{\mathfrak{A}(\mathcal{O})} \underset{E \downarrow 0}{\rightarrow} 0 .
$$

As we show in the next section and in Appendix E the set of theories complying with Condition $C_{\text {七 }}$ contains also massless models, where relation (3.4.24) is a non-trivial statement about the infrared structure. (See the discussion after formula (3.4.6)).

\subsection{Condition $N_{\natural}$ implies Condition $C_{\natural}$}

In the previous section we introduced the phase space condition $C_{\natural}$ and studied it physical consequences. Although this criterion was motivated by the firm physical principle of additivity of energy over isolated subregions, its consistency with the general postulates from Section [1.6 remains to be verified by establishing it in a model. Since the $\varepsilon$-contents of the maps $\Sigma_{E, N, \delta}$ are difficult to control directly in concrete theories, we proceed as follows: We introduce a nuclearity condition $N_{\bullet}$, stated below, which is verified by a relatively straightforward computation in massive and massless scalar free field theory in Appendix E In this section we show that this new condition, which is interesting in its own right, implies Condition $C_{\mathfrak{\natural}}$.

To begin with, let us recall the concept of nuclearity in the form which is suitable for our investigations: Let $V, W$ be Banach spaces and $\|\cdot\|$ be a norm on the space $\mathcal{L}(V, W)$ of linear maps from $V$ to $W$. We say that a map $\Pi: V \rightarrow W$ is $p$-nuclear w.r.t. the norm $\|\cdot\|$, if there exists a decomposition $\Pi(v)=\sum_{n} \Pi_{n}(v)$ into rank-one maps, convergent for any $v \in V$ in the norm topology in $W$, s.t. $\nu:=\left(\sum_{n}\left\|\Pi_{n}\right\|^{p}\right)^{\frac{1}{p}}<\infty$. The $p$-norm $\|\Pi\|_{p}$ of this map is the smallest such $\nu$ over the set of all admissible decompositions. This concept was first used for the description of phase space in quantum field theory in the work of Buchholz and Wichmann BWi86. Our starting point is a stronger nuclearity condition, introduced by Buchholz and Porrmann in BP90:

Condition $N_{\sharp}$ : The maps $\Pi_{E}$ are $p$-nuclear w.r.t. the standard norm on $\mathcal{L}\left(\mathcal{T}_{E}, \mathfrak{A}(\mathcal{O})^{*}\right)$ for any $0<p \leq 1, E \geq 0$ and any double cone $\mathcal{O}$.

For verification of this condition in models, which we present, following Bos00, in Appendix $\left[\mathbb{B}\right.$ it is convenient to have an equivalent formulation in terms of the maps $\Theta_{E}$. It is stated in the following simple lemma. A proof, up to obvious modifications, can be found in BP90. (See also Lemma 3.1.1 above for a similar argument).

Lemma 3.5.1. We fix a double cone $\mathcal{O}$ and $0<p \leq 1$. Then the following conditions are equivalent: 
(a) The maps $\Pi_{E}$ are $p$-nuclear for any $E \geq 0$.

(b) The maps $\Theta_{E}$ are $p$-nuclear for any $E \geq 0$.

Clearly, Condition $N_{\sharp}$ is stronger than Condition $C_{\sharp}$. Thus in theories complying with this nuclearity criterion we can introduce the local continuous subspace $\mathfrak{A}_{\mathrm{c}}(\mathcal{O})=\{A \in$ $\left.\mathfrak{A}(\mathcal{O}) \mid \omega_{0}(A)=0\right\}$, fixed by some energetically accessible vacuum state $\omega_{0}$, similarly as in the first part of Section 3.3. Moreover, we define the maps $\Pi_{E}^{\mathrm{c}} \in \mathcal{L}\left(\mathcal{T}_{E}, \mathfrak{A}_{\mathrm{c}}(\mathcal{O})^{*}\right)$ given by

$$
\Pi_{E}^{\mathrm{c}}(\varphi)=\left.\varphi\right|_{\mathfrak{A}_{\mathrm{c}}(\mathcal{O})},
$$

which satisfy the following identity, valid for any $\varphi \in \mathcal{T}_{E}$ and $A \in \mathfrak{A}(\mathcal{O})$,

$$
\Pi_{E}(\varphi)(A)=\Pi_{E}^{\mathrm{c}}(\varphi)\left(A-\omega_{0}(A) I\right)+\omega_{0}(A) \varphi(I) .
$$

One easily obtains from the above equality that Condition $N_{\sharp}$ holds if and only if there exists an energetically accessible vacuum state and the corresponding maps $\Pi_{E}^{\mathrm{c}}$ are $p$ nuclear for any $0<p \leq 1$. However, we note that this condition is still somewhat conservative, since it does not take into account the fact that for any $\varphi \in \mathcal{T}_{E}$ the restricted functionals $\left.\varphi_{\vec{x}}\right|_{\mathfrak{A}_{\mathrm{c}}(\mathcal{O})}$ should be arbitrarily close to zero apart from translations varying in some compact subset of $\mathbb{R}^{s}$, depending on $\varphi$. It seems therefore desirable to introduce a family of norms on $\mathcal{L}\left(\mathcal{T}_{E}, X\right)$, where $X$ is some Banach space, given for any $N \in \mathbb{N}$ and $x_{1}, \ldots, x_{N} \in \mathbb{R}^{s+1}$ by

$$
\|\Pi\|_{x_{1}, \ldots, x_{N}}=\sup _{\varphi \in \mathcal{T}_{E, 1}}\left(\sum_{k=1}^{N}\left\|\Pi\left(\alpha_{x_{k}}^{*} \varphi\right)\right\|^{2}\right)^{\frac{1}{2}}, \quad \Pi \in \mathcal{L}\left(\mathcal{T}_{E}, X\right)
$$

and the corresponding family of $p$-norms $\|\Pi\|_{p, x_{1}, \ldots, x_{N}}$. It is easily seen that if $\Pi_{E}^{\mathrm{c}}$ is $p$ nuclear w.r.t. the standard norm on $\mathcal{L}\left(\mathcal{T}_{E}, \mathfrak{A}_{\mathrm{c}}(\mathcal{O})\right)$, then it is also $p$-nuclear with respect to the norms (3.5.3) and vice versa. Important additional information is contained in the dependence of the nuclear $p$-norms on $N$. The assumption, which is consistent with the basic postulates and suitable for the purpose of deriving Condition $C_{\sharp}$, is the following:

\section{Condition $N_{\natural}$ :}

(a) There exists an energetically accessible vacuum state $\omega_{0}$ and the corresponding maps $\Pi_{E}^{\mathrm{c}}$ are $p$-nuclear w.r.t. the norms $\|\cdot\|_{x_{1}, \ldots, x_{N}}$ for any $N \in \mathbb{N}, x_{1}, \ldots, x_{N} \in$ $\mathbb{R}^{s+1}, 0<p \leq 1, E \geq 0$ and any double cone $\mathcal{O}$.

(b) The nuclear $p$-norms of the maps $\Pi_{E}^{c}$ satisfy

$$
\limsup \left\|\Pi_{E}^{\mathrm{c}}\right\|_{p, x_{1}, \ldots, x_{N}} \leq c_{p, E},
$$

where $c_{p, E}$ is independent of $N$ and the limit is taken for configurations $x_{1}, \ldots, x_{N}$, where all $x_{i}-x_{j}, i \neq j$, tend to spacelike infinity.

We verify this criterion in massive and massless free field theory, as well as in their even parts, in Appendix E It is clear that if this condition holds in a given theory then it holds also in its sub-theories. In particular it is satisfied by the sub-theory of massless free field theory generated by the derivatives of the field. (See Corollary E.2.6). 
We note as an aside that the quantitative refinement (3.5.4) implies that for any $E \geq 0$, double cone $\mathcal{O}$ and sequence $\{\delta(n)\}_{1}^{\infty}$ s.t. $\delta(n)_{n \rightarrow \infty} \infty$ there holds

$$
\sup _{\substack{\varphi \in \mathcal{T}_{E, 1} \\ A \in \mathfrak{A}_{c}(\mathcal{O})_{1}}} \frac{1}{n} \sum_{i=1}^{n}\left|\varphi\left(A\left(\vec{x}_{i}^{(n)}\right)\right)\right| \leq \frac{1}{\sqrt{n}}\left\|\Pi_{E}^{\mathrm{c}}\right\|_{x_{1}^{(n)}, \ldots, x_{n}^{(n)}} \underset{n \rightarrow \infty}{\rightarrow} 0,
$$

where $\left\{x_{k}^{(n)}\right\}_{1}^{n} \in \Gamma_{n, \delta(n)}$. This formula coincides with relation (3.4.21), so we immediately conclude that Condition $N_{\natural}$ has all the implications listed in Theorem 3.4.3. This approach to the study of the vacuum structure was taken in Dy08.1. However, in the present work we derived properties of the vacuum states from Conditions $C_{\natural}$ and $C_{\mathrm{b}}$ whose physical bases are more solid. Thus Condition $N_{\sharp}$ serves here only as an auxiliary step in the proof that Condition $C_{\natural}$ is consistent with the basic postulates. Our goal in this section is to prove the following theorem:

Theorem 3.5.2. In any quantum field theory Condition $N_{\boxminus}$ implies Condition $C_{\natural}$.

Proceeding towards the proof of this theorem, we first decompose the map $\Sigma_{E, N, \delta} \in$ $\mathcal{L}\left(\mathcal{T}_{E} \times \Gamma_{N, \delta}, \mathfrak{A}(\mathcal{O})^{*} \otimes \mathbb{C}_{\text {sup }}^{N}\right)$, given by (3.4.1), as follows

$$
\begin{aligned}
& \Sigma_{E, N, \delta}=\hat{\Sigma}_{E, N, \delta}+\stackrel{\vee}{\Sigma}_{E, N, \delta}, \\
& \hat{\Sigma}_{E, N, \delta}(\varphi, \underline{\vec{x}})=\left(\Pi_{E}\left(\varphi_{\vec{x}_{1}}\right)-\varphi(I) \omega_{0}, \ldots, \Pi_{E}\left(\varphi_{\vec{x}_{1}}\right)-\varphi(I) \omega_{0}\right), \\
& \stackrel{\vee}{\Sigma}_{E, N, \delta}(\varphi, \underline{\vec{x}})=\varphi(I) \omega_{0} \otimes(1, \ldots, 1) .
\end{aligned}
$$

Since $\stackrel{\vee}{\Sigma}_{E, N, \delta}$ is a rank-one map, its $\varepsilon$-content $\mathcal{V}^{\mathcal{N}}(\varepsilon)_{E, N, \delta}$ satisfies the bound

$$
\stackrel{\vee}{\mathcal{N}}(\varepsilon)_{E, N, \delta} \leq 1+\frac{8^{2}\left\|\stackrel{\vee}{\Sigma}_{E, N, \delta}\right\|^{2}}{\varepsilon^{2}}=1+\frac{8^{2}}{\varepsilon^{2}}
$$

which is independent of $N$. In order to estimate the $\varepsilon$-content $\hat{\mathcal{N}}(\varepsilon)_{E, N, \delta}$ of the map $\hat{\Sigma}_{E, N, \delta}$, we introduce the auxiliary mapping $\Sigma_{E, N, \delta}^{\mathrm{c}} \in \mathcal{L}\left(\mathcal{T}_{E} \times \Gamma_{N, \delta}, \mathfrak{A}_{\mathrm{c}}(\mathcal{O})^{*} \otimes \mathbb{C}_{\text {sup }}^{N}\right)$ given by

$$
\Sigma_{E, N, \delta}^{\mathrm{c}}(\varphi, \underline{\vec{x}})=\left(\Pi_{E}^{\mathrm{c}}\left(\varphi_{\vec{x}_{1}}\right), \ldots, \Pi_{E}^{\mathrm{c}}\left(\varphi_{\vec{x}_{1}}\right)\right) .
$$

We note that for any $\left(\varphi_{1}, \underline{\vec{x}}_{1}\right),\left(\varphi_{2}, \underline{\vec{x}}_{2}\right) \in \mathcal{T}_{E} \times \Gamma_{N, \delta}$ there holds

$$
\left\|\hat{\Sigma}_{E, N, \delta}\left(\varphi_{1}, \underline{\vec{x}}_{1}\right)-\hat{\Sigma}_{E, N, \delta}\left(\varphi_{2}, \underline{\vec{x}}_{2}\right)\right\| \leq 2\left\|\Sigma_{E, N, \delta}^{\mathrm{c}}\left(\varphi_{1}, \underline{\vec{x}}_{1}\right)-\Sigma_{E, N, \delta}^{\mathrm{c}}\left(\varphi_{2}, \underline{\vec{x}}_{2}\right)\right\| .
$$

Thus, by definition of the $\varepsilon$-content, we obtain $\hat{\mathcal{N}}(\varepsilon)_{E, N, \delta} \leq \mathcal{N}_{\mathrm{c}}(\varepsilon / 2)_{E, N, \delta}$, where $\mathcal{N}_{\mathrm{c}}(\varepsilon)_{E, N, \delta}$ is the $\varepsilon$-content of the map $\Sigma_{E, N, \delta}^{c}$. We will control this quantity with the help of Condition $N_{\sharp}$ and the following key lemma.

Lemma 3.5.3. Let $V$ and $W$ be Banach spaces and $\Gamma$ be a set. Let $S_{k} \in \mathcal{L}(V \times \Gamma, \mathbb{C})$ for $k \in\{1, \ldots, N\}$ and $\tau \in W$ be s.t. $\|\tau\|=1$. Then the $\varepsilon$-content of the map $\Sigma \in$ $\mathcal{L}\left(V \times \Gamma, W \otimes \mathbb{C}_{\text {sup }}^{N}\right)$ given by

$$
\Sigma(v, x)=\tau\left(S_{1}(v, x), \ldots, S_{N}(v, x)\right), \quad(v, x) \in V \times \Gamma,
$$

satisfies the bound

$$
\begin{aligned}
& \mathcal{N}(\varepsilon)_{\Sigma} \leq(4 e N)^{\frac{2^{7} \pi\|\Sigma\|_{2}^{2}}{\varepsilon^{2}}}, \\
& \text { where }\|\Sigma\|_{2}=\sup _{(v, x) \in V_{1} \times \Gamma}\left(\sum_{k=1}^{N}\left|S_{k}(v, x)\right|^{2}\right)^{\frac{1}{2}} .
\end{aligned}
$$


Proof. Fix $\varepsilon>0$ and let $\mathbb{J}_{0}=\left\{\left(n_{1}+i n_{2}\right) \varepsilon \mid n_{1}, n_{2} \in \mathbb{Z}\right\}$. For each $k \in\{1, \ldots, N\}$ and $u=(v, x) \in V_{1} \times \Gamma$ we choose $J_{k}(u) \in \mathbb{J}_{0}$ so that $\left|S_{k}(u)-J_{k}(u)\right| \leq \sqrt{2} \varepsilon$ and $\left|J_{k}(u)\right| \leq\left|S_{k}(u)\right|$. Define the set $\mathbb{J}=\left\{J_{1}(u), \ldots, J_{N}(u) \mid u \in V_{1} \times \Gamma\right\}$ of all the $N$-tuples appearing in this way. We claim that $\# \mathbb{J} \geq \mathcal{N}(4 \varepsilon)_{\Sigma}$. In fact, assume that there are $u_{1}, \ldots, u_{K} \in V_{1} \times \Gamma, K>\# \mathbb{J}$, s.t. for $i \neq j$ there holds

$$
4 \varepsilon<\left\|\Sigma\left(u_{i}\right)-\Sigma\left(u_{j}\right)\right\|=\sup _{k \in\{1, \ldots, N\}}\left|S_{k}\left(u_{i}\right)-S_{k}\left(u_{j}\right)\right| .
$$

Then there exists such $\hat{k}$, depending on $(i, j)$, that $4 \varepsilon<\left|S_{\hat{k}}\left(u_{i}\right)-S_{\hat{k}}\left(u_{j}\right)\right|$. Consequently, by a $3 \varepsilon$-argument

$$
\left|J_{\hat{k}}\left(u_{i}\right)-J_{\hat{k}}\left(u_{j}\right)\right| \geq\left|S_{\hat{k}}\left(u_{i}\right)-S_{\hat{k}}\left(u_{j}\right)\right|-2 \sqrt{2} \varepsilon>\varepsilon,
$$

which shows that there are at least $K$ different elements of $\mathbb{J}$ in contradiction to our assumption.

In order to estimate the cardinality of the set $\mathbb{J}$, we define $M=\left[\frac{\|\Sigma\|_{2}^{2}}{\varepsilon^{2}}\right]$, assume for a moment that $0<M \leq 2 N$ and denote by $V_{M}(R) \leq e^{2 \pi R^{2}}$ the volume of the $M$-dimensional ball of radius $R$. Then

$$
\# \mathbb{J} \leq \sum_{\substack{n_{1}, \ldots, n_{2 N} \in \mathbb{Z} \\
n_{1}^{2}+\cdots+n_{2 N}^{2} \leq M}} 1 \leq\left(\begin{array}{c}
2 N \\
M
\end{array}\right) 2^{M} V_{M}(2 \sqrt{M}) \leq(4 N e)^{8 \pi M} .
$$

Here we noted that each admissible combination of integers $n_{1}, \ldots, n_{2 N}$ contains at most $M$ non-zero entries. Thus to estimate the above sum we picked $M$ out of $2 N$ indices and considered the points $\left(n_{i_{1}}, \ldots, n_{i_{M}}\right) \in \mathbb{Z}^{M}$ which belong to the $M$-dimensional ball of radius $\sqrt{M}$. Each such point is a vertex of a unit cube which fits into a ball of radius $2 \sqrt{M}$ (since $\sqrt{M}$ is the length of the diagonal of the cube). As in $M$ dimensions a cube has $2^{M}$ vertices, there can be no more than $2^{M} V_{M}(2 \sqrt{M})$ points $\left(n_{i_{1}}, \ldots, n_{i_{M}}\right) \in \mathbb{Z}^{M}$ satisfying the restriction $n_{i_{1}}^{2}+\cdots+n_{i_{M}}^{2} \leq M$. In the case $M \geq 2 N$ a more stringent bound (uniform in $N$ ) can be established by a similar reasoning. For $M=0$ there obviously holds $\# \mathbb{J}=1$.

Next, by a straightforward combination of Lemma 2.3 and Lemma 2.4 from BD95, we obtain the following useful result which says, essentially, that the $\varepsilon$-content of a sum of maps is equal to the product of their respective $\varepsilon$-contents.

Lemma 3.5.4. Let $V, W$ be Banach spaces, let $\left\{\Sigma_{n}\right\}_{n \in \mathbb{N}}$ be a family of compact maps in $\mathcal{L}(V \times \Gamma, W)$ and $\mathcal{N}(\varepsilon)_{n}$ be the corresponding $\varepsilon$-contents. Suppose that $\sum_{n}\left\|\Sigma_{n}\right\|<\infty$. Then the $\varepsilon$-content $\mathcal{N}(\varepsilon)$ of the (compact) map $\Sigma:=\sum_{n} \Sigma_{n}$ satisfies, for any sequence of positive numbers $\left\{\varepsilon_{n}\right\}_{n \in \mathbb{N}}$ s.t. $\sum_{n} \varepsilon_{n} \leq \varepsilon / 4$, the following bound

$$
\mathcal{N}(\varepsilon) \leq \liminf _{k \rightarrow \infty} \prod_{n=1}^{k} \mathcal{N}\left(\varepsilon_{n}\right)_{n} .
$$

After this preparation we are ready to prove Theorem 3.5.2. The argument relies on techniques from the proof of Proposition 2.5 (ii) in BD95].

Proof of Theorem [3.5.2; Fix $0<p<\frac{2}{3}$. Then Condition $N_{\natural}$ provides, for any 
$\gamma>0$, a decomposition of the map $\Pi_{E}^{\mathrm{c}}$ into rank-one mappings $\Pi_{n}(\cdot)=\tau_{n} S_{n}(\cdot)$, where $\tau_{n} \in \mathfrak{A}_{\mathrm{c}}(\mathcal{O})^{*}$ and $S_{n} \in \mathcal{T}_{E}^{*}$, s.t.

$$
\left(\sum_{n=1}^{\infty}\left\|\Pi_{n}\right\|_{x_{1}, \ldots, x_{N}}^{p}\right)^{\frac{1}{p}} \leq(1+\gamma)\left\|\Pi_{E}^{\mathrm{c}}\right\|_{p, x_{1}, \ldots, x_{N}} .
$$

Assuming that the norms $\left\|\Pi_{n}\right\|_{x_{1}, \ldots, x_{N}}$ are given in descending order with $n$, we obtain the bound

$$
\left\|\Pi_{n}\right\|_{x_{1}, \ldots, x_{N}} \leq \frac{(1+\gamma)\left\|\Pi_{E}^{\mathrm{c}}\right\|_{p, x_{1}, \ldots, x_{N}}}{n^{1 / p}} .
$$

Similarly, we can decompose the map $\Sigma_{E, N, \delta}^{c}$, given by (3.5.10), into a sum of maps $\Sigma_{n} \in \mathcal{L}\left(\mathcal{T}_{E} \times \Gamma_{N, \delta}, \mathfrak{A}_{\mathrm{c}}(\mathcal{O})^{*} \otimes \mathbb{C}_{\text {sup }}^{N}\right)$ of the form

$$
\Sigma_{n}(\varphi, \underline{\vec{x}})=\left(\Pi_{n}\left(\varphi_{\vec{x}_{1}}\right), \ldots, \Pi_{n}\left(\varphi_{\vec{x}_{N}}\right)\right)=\tau_{n}\left(S_{n}\left(\varphi_{\vec{x}_{1}}\right), \ldots, S_{n}\left(\varphi_{\vec{x}_{N}}\right)\right) .
$$

Now we apply Lemma 3.5 .3 with $\tau=\tau_{n} /\left\|\tau_{n}\right\|$ and $S_{k} \in \mathcal{L}\left(\mathcal{T}_{E} \times \Gamma_{N, \delta}, \mathbb{C}\right), k \in\{1, \ldots, N\}$ given by

$$
S_{k}(\varphi, \underline{\vec{x}})=\left\|\tau_{n}\right\| S_{n}\left(\varphi_{\vec{x}_{k}}\right) .
$$

From estimate (3.5.19) we obtain

$$
\begin{aligned}
\left\|\Sigma_{n}\right\|_{2} & =\sup _{(\varphi, \underline{\vec{x}}) \in \mathcal{T}_{E, 1} \times \Gamma_{N, \delta}}\left(\sum_{k=1}^{N}\left\|\tau_{n}\right\|^{2}\left|S_{n}\left(\varphi_{\vec{x}_{k}}\right)\right|^{2}\right)^{\frac{1}{2}} \\
& =\sup _{\underline{\vec{x}} \in \Gamma_{N, \delta}}\left\|\Pi_{n}\right\|_{x_{1}, \ldots, x_{N}} \leq \sup _{\underline{\vec{x}} \in \Gamma_{N, \delta}} \frac{(1+\gamma)\left\|\Pi_{E}^{\mathrm{c}}\right\|_{p, x_{1}, \ldots, x_{N}}}{n^{1 / p}},
\end{aligned}
$$

where $x_{k}=\left(0, \vec{x}_{k}\right), k \in\{1, \ldots, N\}$. Substituting this inequality to the bound (3.5.13) we get

$$
\mathcal{N}(\varepsilon)_{n} \leq(4 e N) \frac{2^{7} \pi(1+\gamma)^{2}\left(\sup _{\left.\underline{\underline{\underline{x}}} \in \Gamma_{N, \delta}\left\|\Pi_{E}\right\|_{p, x_{1}, \ldots, x_{N}}^{2}\right)}\right.}{\varepsilon^{2} n^{2 / p}} .
$$

Since $\left\|\Sigma_{n}\right\|=\left\|\Pi_{n}\right\|$, it follows from (3.5.18) that the summability assumption of Lemma 3.5.4 is satisfied. We choose $\varepsilon_{n}=\frac{\varepsilon}{4} \frac{n^{-2 /(3 p)}}{\sum_{n_{1}=1}^{\infty} n_{1}^{-2 /(3 p)}}$, make use of the bounds (3.5.23) and (3.5.17), and take the infinum w.r.t. $\gamma>0$. There follows

$$
\mathcal{N}_{\mathrm{C}}(\varepsilon)_{E, N, \delta} \leq(4 e N) \frac{2^{11} \pi\left(\sup _{\underline{\underline{x}} \in \Gamma_{N, \delta}}\left\|\Pi_{E}\right\|_{p, x_{1}, \ldots, x_{N}}^{2}\right)}{\varepsilon^{2}}\left(\sum_{n=1}^{\infty} n^{-2 /(3 p)}\right)^{3} .
$$

From decomposition (3.5.6), estimate (3.5.9) and Lemma 3.5.4 we obtain the bound on the $\varepsilon$-content of the map $\Sigma_{E, N, \delta}$

$$
\mathcal{N}(\varepsilon)_{E, N, \delta} \leq \mathcal{N}_{\mathrm{c}}(\varepsilon / 8)_{E, N, \delta} \mathcal{V}_{\mathrm{c}}^{\vee}(\varepsilon / 8)_{E, N, \delta} \leq \mathcal{N}_{\mathrm{c}}(\varepsilon / 8)_{E, N, \delta}\left(1+\frac{8^{4}}{\varepsilon^{2}}\right)
$$

The 1.h.s. of this inequality is a decreasing and bounded from below function of $\delta$, so we can take the limit $\delta \rightarrow \infty$. With the help of formula (3.5.24) and Condition $N_{\sharp}$ we obtain the bound (3.4.8) in the statement of Condition $C_{\mathfrak{t}}$. 


\section{Chapter 4}

\section{Conclusions and Outlook}

In this work we have developed a detailed spectral theory of translation automorphisms in quantum field theory, pursuing the programme initiated in Bu90. Motivated by the spectral properties of unitary representations of translations acting on a Hilbert space, we proposed a decomposition of the algebra of local observables $\hat{\mathfrak{A}}$ into subspaces which differ in their behavior under translations in space. Our investigation has led to new insights into the infrared structure of quantum field theories, their particle content and properties of the vacuum states.

First, we identified the counterpart of the pure-point subspace. In order to find the natural projection $P_{\{0\}}$ on this subspace in the absence of orthogonality, we have established a variant of the ergodic theorem for the space translation automorphisms. It relies on physically motivated phase space conditions, whose consistency with the basic postulates has been verified by explicit computations in models of non-interacting particles. We have shown that these criteria have, in addition, a number of consequences pertaining to the vacuum structure, including the uniqueness of the energetically accessible vacuum state $\omega_{0}$ and relaxation of physical states to $\omega_{0}$ under large timelike translations. Moreover, this vacuum state is simply related to the above mentioned projection: $P_{\{0\}}(\cdot)=\omega_{0}(\cdot) I$. The continuous subspace has been defined as the kernel of this projection, or equivalently $\hat{\mathfrak{A}}_{\mathrm{c}}=\operatorname{ker} \omega_{0}$.

Proceeding to a more detailed spectral analysis, we took the quantum-mechanical framework as a guide: We noted that the square-integrability of the transition amplitude between a vector and its translate implies that this vector belongs to the absolutely continuous subspace. We convinced ourselves that this property always holds for 'local' vectors whose wavefunctions are compactly supported in configuration space, which serve as analogues of local operators. More importantly, transition amplitudes between an arbitrary vector and translates of a 'local' one are also square-integrable, but not necessarily integrable with any smaller power. Summing up, the square-integrability of the transition amplitudes on the one hand implies the absolute continuity of the spectrum and on the other hand is the strongest decay property to be expected from 'local' vectors. Therefore, in quantum field theory we have defined the absolutely continuous subspace $\hat{\mathfrak{A}}_{\mathrm{ac}}$ as consisting of square-integrable elements i.e. $A \in \hat{\mathfrak{A}}_{\mathrm{c}}$ satisfying $\|A\|_{E, 2}<\infty$ for any $E \geq 0$. We have shown that in a number of cases this subspace can be expressed as the intersection of kernels of a finite family of linear functionals i.e. $\hat{\mathfrak{A}}_{\mathrm{ac}}=\operatorname{ker} \omega_{0} \cap \operatorname{ker} \tau_{1} \cap \ldots \cap \operatorname{ker} \tau_{n}$. Then its direct sum complement is finite dimensional, akin to the pure-point part, what motivates the term 'point-continuous subspace' $\hat{\mathfrak{A}}_{\mathrm{pc}}$. We emphasize that this subspace does not have 
a quantum mechanical counterpart - it carries information about the infrared structure which is specific to quantum field theories. If $\hat{\mathfrak{A}}_{\mathrm{pc}}$ is non-trivial, then $\hat{\mathfrak{A}}_{\mathrm{c}}$ contains some elements $A$ which are not square-integrable. Their deviation from square-integrability can be quantified with the help of a new concept: the 'infrared order' $\operatorname{ord}(A)$ of the observable $A$. It captures the regularity properties of the distribution $\widetilde{A}(\vec{p})$ which, as we argued in Section 1.4 is a natural analogue of the spectral measure for the Arveson spectrum. Since the infrared orders of observables from $\hat{\mathfrak{A}}_{\mathrm{c}}$ can also be computed with the help of the functionals $\left\{\tau_{j}\right\}_{1}^{n}$ (see the proof of Theorem 2.2.5 in Section C.1), a model-independent construction of such functionals would constitute a major progress in our understanding of the spectral theory of automorphism groups. Taking our study of non-interacting examples as a guide, decent phase space properties and sufficiently rich field content of a theory should be relevant to the study of this problem.

As explained in Section 1.3, to unravel particle aspects of a theory, one needs integrable observables i.e. such $C \in \mathfrak{A}$ that $\|C\|_{E, 1}<\infty$ for any $E \geq 0$. In view of the above discussion we conjecture that there do not exist non-zero local operators which satisfy this property. A class of almost local, integrable observables was found in Bu90. However, apart from the case of Wigner particles, it was not known under what conditions the particle content of a theory is non-trivial. We have proposed a criterion, suitable for a class of massive theories, which introduces a new family of particle detectors. With these novel integrable observables one can approximate pointlike localized fields in the topology generated by the seminorms $\|\cdot\|_{E, 1}$. Assuming in addition the existence of the stressenergy tensor, we have obtained non-triviality of the particle content, substantiating the strategy put forward in Bu94. We expect that the conditions mentioned above, or their strengthened variants, have other interesting consequences, perhaps even some weak form of asymptotic completeness, as discussed in the last part of Section 2.3 It would therefore be desirable to find similar criteria in the realm of massless theories. There we have acquired thorough understanding of the square-integrability properties of observables, but integrable detectors, which could approximate the stress-energy tensor, have not been found as yet.

Another interesting problem is the convergence of the asymptotic functional approximants $\left\{\sigma_{\varphi}^{(t)}\right\}_{t \in \mathbb{R}_{+}}$, defined by formula (2.3.6), as $t \rightarrow \infty$. This property is certainly expected on physical grounds, since the results of particle physics experiments stabilize for sufficiently large times. Although we do not know any examples, where this property would fail, a proof relying only on the basic postulates of QFT is out of sight at the moment. On the other hand, our study of the problem of convergence to the vacuum in Chapter 3 suggests an approach based on phase space conditions. Let us outline briefly a possible strategy: First, we note that Condition $C_{\natural}$, stated in Section 3.4, provides a promising starting point as it does not require any a priori knowledge of the limiting state. This is a great advantage in the present context, since the asymptotic functionals $\sigma_{\varphi}^{(+)}$, in contrast to the asymptotic vacuum states, depend in a complicated manner on the initial functional $\varphi$. In fact, they should describe all the possible asymptotic particle configurations, so no uniqueness result is expected here. To reformulate Condition $C_{\natural}$ so that it describes the results of particle measurements, separated by large time intervals, we define

$$
\Gamma_{N, \delta}^{(+)}=\left\{\underline{t}:=\left(t_{1}, \ldots, t_{N}\right) \in \mathbb{R}_{+}^{N}|| t_{i}-t_{j} \mid \geq \delta \text { for } i \neq j\right\} .
$$

Next, we adopt Condition $L^{(1)}$ stated in Section 2.3] and note that $\mathfrak{A}_{\mathrm{c}}(\mathcal{O}) \ni A \rightarrow \sigma_{\varphi}^{(t)}(A(g))$ 
is a bounded linear functional for any $t \in \mathbb{R}_{+}, \varphi \in \mathcal{T}_{E}$ and a suitable time-smearing function $g \in S(\mathbb{R})$. It is convenient to shift the smearing on the functional $\varphi$ i.e. to write $\sigma_{\varphi_{g}}^{(t)}(A)$ instead of $\sigma_{\varphi}^{(t)}(A(g))$. Now we introduce the maps $\Sigma_{E, N, \delta}^{(+)}: \mathcal{T}_{E} \times \Gamma_{N, \delta}^{(+)} \rightarrow \mathfrak{A}_{\mathrm{c}}(\mathcal{O})^{*} \otimes \mathbb{C}_{\text {sup }}^{N}$ given by

$$
\Sigma_{E, N, \delta}^{(+)}(\varphi, \underline{t})=\left(\sigma_{\varphi_{g}}^{\left(t_{1}\right)}, \ldots, \sigma_{\varphi_{g}}^{\left(t_{N}\right)}\right)
$$

The limits of $\left\{\sigma_{\varphi_{g}}^{(t)}\right\}_{t \in \mathbb{R}_{+}}$as $t \rightarrow \infty$ are directly related to the asymptotic functionals which constitute the particle content of the theory. Thus in physical terms the ranges of these maps consist of collections of data (summed over time-slices) obtained in particle physics experiments. In a typical experiment, after a finite number of rescattering events there emerges a stable, asymptotic configuration. (See [Ha, Section VI.2.3). As a first orientation, let us make the 'uniform dispersion' assumption which says that the number of such events, which can be detected up to some accuracy $\varepsilon$, is bounded by some constant $c(\varepsilon, E)$, independent of the initial functional $\varphi \in \mathcal{T}_{E, 1}$. Hence we obtain that the number of distinguishable collections of data should not exceed $(\underset{c(\varepsilon, E)}{N}) \leq N^{c(\varepsilon, E)}$. Therefore, we impose on the maps $\Sigma_{E, N, \delta}^{(+)}$the following variant of Condition $C_{\natural}$ :

\section{Tentative Condition $C_{\natural}^{(+)}$:}

(a) The maps $\Sigma_{E, N, \delta}^{(+)}$are compact for any $E \geq 0, N \in \mathbb{N}, \delta>0$ and any double cone $\mathcal{O} \subset \mathbb{R}^{s+1}$.

(b) The $\varepsilon$-contents $\mathcal{N}(\varepsilon)_{E, N, \delta}^{(+)}$of the maps $\Sigma_{E, N, \delta}^{(+)}$satisfy, for any $\varepsilon>0$,

$$
\lim _{\delta \rightarrow \infty} \mathcal{N}(\varepsilon)_{E, N, \delta}^{(+)} \leq(N+1)^{c(E, \varepsilon)},
$$

for some constant $c(E, \varepsilon)$ independent of $N$.

Under this condition we immediately obtain the convergence of the asymptotic functional approximants on the class of detectors introduced in Condition $L^{(1)}$ : We fix $\varphi \in \mathcal{T}_{E, 1}$. By the compactness of the map $\Sigma_{E, 1, \delta}^{(+)}$, there exist limit points $\sigma_{\varphi g}^{(i,+)}$ of the net $\left\{\sigma_{\varphi_{g}}^{(t)}\right\}_{t \in \mathbb{R}_{+}}$ as $t \rightarrow \infty$, which can be approximated by sequences $\left\{\sigma_{\varphi_{g}}^{\left(t_{i, n}\right)}\right\}_{n \in \mathbb{N}}$ in the norm topology of $\mathfrak{A}_{\mathrm{c}}(\mathcal{O})^{*}$. Suppose that there are two different limit points $\sigma_{\varphi_{g}}^{(1,+)}$ and $\sigma_{\varphi_{g}}^{(2,+)}$ i.e. there holds $\left|\sigma_{\varphi_{g}}^{(1,+)}(A)-\sigma_{\varphi_{g}}^{(2,+)}(A)\right|>\varepsilon$ for some $A \in \mathfrak{A}_{\mathrm{c}}(\mathcal{O})_{1}$ and $\varepsilon>0$. By choosing suitable subsequences of their approximating sequences, one can easily show that for any $i_{1}, \ldots, i_{N} \in\{1,2\}$ the elements

$$
\left(\sigma_{\varphi_{g}}^{\left(i_{1},+\right)}, \ldots, \sigma_{\varphi_{g}}^{\left(i_{N},+\right)}\right) \in \mathfrak{A}_{\mathrm{c}}(\mathcal{O})^{*} \otimes \mathbb{C}_{\mathrm{sup}}^{N}
$$

belong to the closures of the ranges of the maps $\Sigma_{E, N, \delta}^{(+)}$for any $\delta>0$. Since there are $2^{N}$ such elements and their mutual norm distances are larger than $\varepsilon$, the bound (4.0.3) is violated. We conclude that all the limit points $\sigma_{\varphi g}^{(i,+)}$ are equal. Thus, for any $\varphi \in \mathcal{T}_{E}$, we obtain the unique functional $\sigma_{\varphi_{g}}^{(+)}=\lim _{t \rightarrow \infty} \sigma_{\varphi_{g}}^{(t)}$ describing the asymptotic particle configuration. Moreover, by part (a) of the above assumption, the set $\left\{\sigma_{\varphi_{g}}^{(+)} \mid \varphi \in \mathcal{T}_{E, 1}\right\}$ is compact in the norm topology of $\mathfrak{A}_{\mathrm{c}}(\mathcal{O})^{*}$. 
Unfortunately, we do not have a complete verification argument for the above tentative condition. Preliminary computations in massive scalar free field theory confirm the qualitative part (a). On the other hand, the quantitative refinement (b) seems to be satisfied only after replacing the space $\mathfrak{A}_{\mathrm{c}}(\mathcal{O})$ with a smaller, but infinitely dimensional subspace $\mathfrak{A}_{\mathrm{c}}(\mathcal{O})^{(+)}$in the definition of the maps $\Sigma_{E, N, \delta}^{(+)}$. This indicates that our 'uniform dispersion' picture can be maintained only after excluding some 'oversensitive' detectors. In this particular model it can be shown that the subspace $\mathfrak{A}_{c}(\mathcal{O})^{(+)}$is sufficiently large to ensure non-triviality of the asymptotic functionals, but it is not clear how to formulate this requirement in general terms. Interestingly, $\mathfrak{A}_{\mathrm{c}}(\mathcal{O})^{(+)}$can be expressed as the intersection of kernels of a (countable) family of functionals, akin to the spectral subspaces discussed above. Better understanding of the origin of this subspace may, on the one hand, shed light on the question of convergence of the asymptotic functional approximants, on the other hand open the door to deeper understanding of the spectral theory of automorphism groups. 


\section{Appendix A}

\section{Haag-Ruelle Scattering Theory in Presence of Massless Particles}

In this appendix, which complements our discussion in Section 1.2 we construct a scattering theory of stable, massive particles without assuming mass gaps. This extension of the Haag-Ruelle theory is based on advances in the harmonic analysis of local operators [Bu90] restated in Theorem 1.6.2 of the present Thesis. Our construction is restricted to theories complying with a regularity property introduced by Herbst, stated as Condition $A^{\prime}$ below. The appendix concludes with a brief discussion of the status of this assumption. The analysis presented here has been published in Dy05.

\section{A.1 Introduction}

It is the aim of this appendix to prove that the Haag-Ruelle collision theory can be extended to stable massive particles, obeying a sharp dispersion law, in the presence of massless excitations. Thus we do not touch upon the infraparticle problem [Sch63, Bu86], mentioned in Section 1.2, but our arguments are applicable, for example, to electrically neutral, stable particles such as atoms in quantum electrodynamics. Before we enter into this discussion we briefly outline our notation, state our assumptions and comment on previous approaches to this problem.

Similarly as in Section 1.2, we adopt here a more restrictive framework than the one expounded in Section [1.6. First, we restrict attention to the physical case of dimension of space $s=3$. Next, we assume that the Poincaré transformations are unitarily implemented i.e. there exists a continuous unitary representation $P_{+}^{\uparrow} \ni(x, \Lambda) \rightarrow U(x, \Lambda)$ acting on the Hilbert space $\mathcal{H}$ s.t. $\alpha_{(x, \Lambda)}(\cdot)=U(x, \Lambda) \cdot U(x, \Lambda)^{*}$. It is also required that there exists in $\mathcal{H}$ a unique (up to a phase) vacuum vector $\Omega$ which is invariant under the action of $U$. Finally, we suppose that the point spectrum of the mass operator $M=\sqrt{H^{2}-\vec{P}^{2}}$ consists, apart from 0 , of a unique eigenvalue $m>0$. We also postulate that the representation $U$, restricted to the corresponding spectral subspace $\mathcal{H}^{[m]}$, coincides with the irreducible representation of the Poincaré group of mass $m$ and spin 0 . In other words, we consider a single species of massive, spinless particles. In the pioneering work of Haag [Ha58] and Ruelle Ru62 these general postulates were amended by two additional requirements:

Condition $A$ : The time-dependent operators $A\left(f_{T}\right)=\int A(x) f_{T}(x) d^{4} x$, constructed from $A(x)=U(x) A U(x)^{-1}, A \in \mathfrak{A}(\mathcal{O})$ and suitably chosen sequences of functions 
$f_{T} \in S\left(\mathbb{R}^{4}\right)$, satisfy $A\left(f_{T}\right) \Omega \neq 0, A\left(f_{T}\right) \Omega \in \mathcal{H}^{[m]}$ and $\frac{d}{d T} A\left(f_{T}\right) \Omega=0$.

Condition $M$ : The vacuum is isolated from the rest of the energy-momentum spectrum.

Both of these conditions are ensured if the mass $m$ is an isolated eigenvalue of the mass operator $M$. On the other hand, if the mass of the particle in question is an embedded eigenvalue, then it seems difficult to meet the requirement $A$. It was, however, noticed by Herbst Her71] that, in fact, it is only needed in the proof that $\mathrm{s}-\lim _{T \rightarrow \infty} A\left(f_{T}\right) \Omega$ is a nonzero vector in $\mathcal{H}^{[m]}$ and $\left\|\frac{d}{d T} A\left(f_{T}\right) \Omega\right\|$ is an integrable function of $T$. We summarize here Herbst's analysis since it will be the starting point of our considerations: The operators $A\left(f_{T}\right)$ are constructed in a slightly different manner than in the work of Haag and Ruelle: First, a local operator $A$ is smeared in space with a regular solution of the Klein-Gordon equation $f(t, \vec{x})=\frac{1}{(2 \pi)^{3 / 2}} \int e^{-i \omega(\vec{p}) t+i \vec{p} \vec{x}} \tilde{f}(\vec{p}) d^{3} p,\left(\right.$ where $\tilde{f} \in C_{0}^{\infty}\left(\mathbb{R}^{s}\right), \omega(\vec{p})=\sqrt{\vec{p}^{2}+m^{2}}$ )

$$
A_{t}(f)=\int A(t, \vec{x}) f(t, \vec{x}) d^{3} x
$$

Next, to construct the time averaging function, we choose $s(T)=T_{\tilde{h}}^{\nu}, 0<\nu<1$ and a positive function $h \in S(\mathbb{R})$ such that its Fourier transform satisfies $\tilde{h} \in C_{0}^{\infty}(\mathbb{R}), \tilde{h}(0)=$ $(2 \pi)^{-2}$. Then we set $h_{T}(t)=\frac{1}{s(T)} h\left(\frac{t-T}{s(T)}\right)$ for $T \geq 1$ and define Her71, Bu77]

$$
A\left(f_{T}\right)=\int h_{T}(t) A_{t}(f) d t
$$

It is clear from formulas A.1.1 and A.1.2 that $f_{T}(x)=h_{T}\left(x^{0}\right) f\left(x^{0}, \vec{x}\right)$. Its Fourier transform $\tilde{f}_{T}$ has a compact support which approaches a compact subset of the mass hyperboloid as $T \rightarrow \infty$. In view of this fact we will refer to $A\left(f_{T}\right)$ as creation operators and to $A\left(f_{T}\right)^{*}$ as annihilation operators. This terminology is also supported by the following simple calculation

$$
\mathrm{s}-\lim _{T \rightarrow \infty} A\left(f_{T}\right) \Omega=P_{[m]} A(f) \Omega,
$$

where $A(f)=A_{t=0}(f)$ and $P_{[m]}$ is the projection on the single-particle space $\mathcal{H}^{[m]}$. The integrability condition requires the following assumption:

Condition $A^{\prime}$ : There exist operators $A \in \mathfrak{A}(\mathcal{O})$ such that $P_{[m]} A \Omega \neq 0$ and for every $\delta \geq 0$

$$
\left\|P\left(m^{2}-\delta \leq p^{2} \leq m^{2}+\delta\right)\left(1-P_{[m]}\right) A \Omega\right\| \leq c \delta^{\varepsilon},
$$

where $P(\cdot)$ is the spectral measure of the energy-momentum operators and $c, \varepsilon>0$. We refer to such operators as 'regular'.

For regular operators there holds the bound

$$
\left\|\frac{d}{d T} A\left(f_{T}\right) \Omega\right\| \leq \frac{c}{s(T)^{1+\varepsilon}}
$$

which implies integrability if $\nu>\frac{1}{1+\varepsilon}$. Now we are ready to state the main result of Herbst; we restrict attention to the outgoing asymptotic states $\Psi^{+}$, since the case of incoming states is completely analogous. 
Theorem A.1.1. Her71 Suppose that the theory respects Conditions $M$ and $A^{\prime}$. Then, for regular operators $A_{i}, i \in\{1, \ldots, n\}$, there exists the limit

$$
\Psi^{+}=s-\lim _{T \rightarrow \infty} A_{1}\left(f_{1 T}\right) \ldots A_{n}\left(f_{n T}\right) \Omega
$$

and it depends only on the single-particle states $P_{[m]} A_{i}\left(f_{i}\right) \Omega$. Moreover, given two states $\Psi^{+}$and $\hat{\Psi}^{+}$, constructed as above using creation operators $A_{i}\left(f_{i, T}\right)$ and $\widehat{A}_{i}\left(\widehat{f}_{i, T}\right), i \in$ $\{1, \ldots, n\}$, their scalar product can be calculated as follows

$$
\left(\Psi^{+} \mid \widehat{\Psi}^{+}\right)=\sum_{\sigma \in S_{n}}\left(\Omega \mid A_{1}\left(f_{1}\right)^{*} P_{[m]} \widehat{A}_{\sigma_{1}}\left(\widehat{f}_{\sigma_{1}}\right) \Omega\right) \ldots\left(\Omega \mid A_{n}\left(f_{n}\right)^{*} P_{[m]} \widehat{A}_{\sigma_{n}}\left(\widehat{f}_{\sigma_{n}}\right) \Omega\right) .
$$

Here the sum is over all permutations of an n-element set.

It was, however, anticipated already by Ruelle $\mathrm{Ru} 62$ that in a purely massive theory Condition $A$ can be replaced by the following, physically meaningful, stability requirement

Condition $S$ : In a theory satisfying $\mathrm{M}$ a particle can only be stable if, in its superselection sector, its mass is separated from the rest of the spectrum by a lower and upper mass gap.

This condition is also stated in Herbst's work Her71, but he expects that scattering theory should be a necessary tool to study the superselection structure. Subsequent analysis by Buchholz and Fredenhagen BF82 clarified this issue: There exist interpolating fields which connect the vacuum with the sector of the given particle. Although they are, in general, localized in spacelike cones, they can be used to construct a collision theory. Thereby there exists a prominent alternative to the approach of Herbst in the realm of massive theories.

It is the purpose of our investigations to extend Herbst's result to the situation where massless particles are present, that is Conditions $M$ and $S$ do not hold. A model physical example of a system with a sharp mass immersed in a spectrum of massless particles is the hydrogen atom in its ground state from the point of view of quantum electrodynamics. Although the approach of Herbst seems perfectly adequate to study such situations, the original proof of Theorem A.1.1 does not work because of the slow, quadratic decay of the correlation functions. In order to overcome this difficulty, we apply the bounds on creation operators obtained by Buchholz [Bu90]. Namely, if $\Delta$ is any compact subset of the energy-momentum spectrum and $\tilde{f}$ vanishes sufficiently fast at zero then

$$
\begin{aligned}
\left\|A\left(f_{T}\right) P(\Delta)\right\| & \leq c, \\
\left\|A\left(f_{T}\right)^{*} P(\Delta)\right\| & \leq c,
\end{aligned}
$$

where the constant $c$ does not depend on time. In fact, these bounds easily follow from Theorem 1.6.2 First, we note that by the Cauchy-Schwarz inequality there holds for any $\varepsilon>0, E \geq 0$

$$
\left\|P_{E} A_{t}(f) P_{E}\right\| \leq \sup _{\varphi \in \mathcal{T}_{E, 1}}\left(\int d^{3} p|\vec{p}|^{4+\varepsilon}|\varphi(\widetilde{A}(\vec{p}))|^{2}\right)^{\frac{1}{2}}\left(\int d^{3} p \frac{1}{|\vec{p}|^{4+\varepsilon}}|\tilde{f}(\vec{p})|^{2}\right)^{\frac{1}{2}} .
$$

In particular, the r.h.s. is independent of $t$. Next, making use of the fact that the Fourier transform of $f_{T}$ has a compact support, we can find for any compact set $\Delta \subset \mathbb{R}^{4}$ another compact subset $\Delta^{\prime}$ of the energy-momentum spectrum and $E \geq 0$ s.t.

$$
\left\|A\left(f_{T}\right) P(\Delta)\right\|=\left\|P\left(\Delta^{\prime}\right) A\left(f_{T}\right) P(\Delta)\right\| \leq\|h\|_{1} \sup _{t \in \mathbb{R}}\left\|P_{E} A_{t}(f) P_{E}\right\| .
$$


This bound together with estimate (A.1.10) justifies relation (A.1.8). The case of $A\left(f_{T}\right)^{*}$ is treated analogously.

The remaining part of this appendix is organized as follows: In Section A.2 we prove the existence of asymptotic states and verify that the limits are independent of the actual value of the parameter $0<\nu<1$ chosen in the time averages of the operators $A\left(f_{T}\right)$. This property allows us to apply in Section A.3 the methods from the collision theory of massless bosons [Bu77] in order to calculate the scalar product of asymptotic states. In the Conclusion we summarize our results and discuss the status of Condition $A^{\prime}$.

\section{A.2 Existence of Asymptotic States}

In order to prove the existence of the asymptotic states we need information about the time evolution of the operators $A\left(f_{T}\right)$ and their commutators. It is the purpose of the two lemmas below to summarize the necessary properties. Before we enter into these investigations, we recall that the regular solutions of the Klein-Gordon equation satisfy the bounds Ru62]

$$
\begin{aligned}
\left|f_{t}(\vec{x})\right| & \leq c(1+|t|)^{-3 / 2}, \\
\int d^{3} x\left|f_{t}(\vec{x})\right| & \leq c(1+|t|)^{3 / 2},
\end{aligned}
$$

where the constant $c$ is independent of $t$ and $\vec{x}$.

Lemma A.2.1. Let $A\left(f_{T}\right)^{\#}$ denote $A\left(f_{T}\right)$ or $A\left(f_{T}\right)^{*}$. Then:

(a) $\left\|A\left(f_{T}\right)^{\#}\right\| \leq c T^{3 / 2}$.

(b) $P\left(\Delta_{1}\right) A\left(f_{T}\right)^{\#} P\left(\Delta_{2}\right)=0$ if $\Delta_{1} \cap\left(\Delta_{2} \pm\right.$ supp $\left.\tilde{f}_{T}\right)=\emptyset$. The (+) sign holds for $A\left(f_{T}\right)$, $(-)$ for $A\left(f_{T}\right)^{*}$.

(c) Suppose that the functions $\tilde{f}_{i}, i \in\{1, \ldots, n\}$, vanish sufficiently fast at zero. Then, for any compact subset $\Delta$ of the energy-momentum spectrum:

$$
\left\|A_{1}\left(f_{1, T}\right)^{\#} \ldots A_{n}\left(f_{n, T}\right)^{\#} P(\Delta)\right\| \leq c_{1} .
$$

The constants $c, c_{1}$ do not depend on $T$.

\section{Proof.}

(a) The statement follows from the estimate

$$
\begin{aligned}
\left\|A\left(f_{T}\right)^{\#}\right\| & \leq\left\|A^{\#}\right\| \int d t h_{T}(t) \int d^{3} x|f(t, \vec{x})| \leq c_{0} \int d t h_{T}(t)(1+|t|)^{3 / 2} \\
& =c_{0} \int d t h(t)(1+|s(T) t+T|)^{3 / 2} \leq c T^{3 / 2}
\end{aligned}
$$

where in the second step we used property (A.2.1) of regular solutions of the KleinGordon equation.

(b) See, for example, $\operatorname{Ar} 82$. 
(c) For $n=1$ the assertion follows from (A.1.8) and A.1.9). Assuming that A.2.3 is valid for $n-1$ and making use of part (b) of this lemma we estimate

$$
\begin{aligned}
& \left\|A_{1}\left(f_{1, T}\right)^{\#} \ldots A_{n}\left(f_{n, T}\right)^{\#} P(\Delta)\right\| \\
= & \left\|A_{1}\left(f_{1, T}\right)^{\#} \ldots A_{n-1}\left(f_{n-1, T}\right)^{\#} P\left(\Delta \pm \operatorname{supp} \tilde{f}_{n, T}\right) A_{n}\left(f_{n, T}\right)^{\#} P(\Delta)\right\| \\
\leq & \left\|A_{1}\left(f_{1, T}\right)^{\#} \ldots A_{n-1}\left(f_{n-1, T}\right)^{\#} P\left(\Delta \pm \operatorname{supp} \tilde{f}_{n, T}\right)\right\|\left\|A_{n}\left(f_{n, T}\right)^{\#} P(\Delta)\right\| .(\text { A. } 2.5)
\end{aligned}
$$

The last expression is bounded by the inductive assumption and the support properties of functions $\tilde{f}_{T}$.

Now we turn our attention to the commutators of the operators $A\left(f_{T}\right)$. It will simplify this discussion to decompose the function $f_{T}$ into its compactly supported dominant contribution and a spatially extended, but rapidly decreasing remainder [BBS01]. To this end, let us define the velocity support of the function $\tilde{f}$

$$
\Gamma(\tilde{f})=\left\{\left(1, \frac{\vec{p}}{\omega(\vec{p})}\right) \mid \vec{p} \in \operatorname{supp} \tilde{f}\right\}
$$

We introduce a function $\chi_{\delta} \in C_{0}^{\infty}\left(\mathbb{R}^{4}\right)$ such that $\chi_{\delta}=1$ on $\Gamma(\tilde{f})$ and $\chi_{\delta}=0$ in the complement of a slightly larger set $\Gamma(\tilde{f})_{\delta} . \hat{f}_{T}(x)=f_{T}(x) \chi_{\delta}(x / T)$ is the asymptotically dominant part of $f_{T}$, whereas $\stackrel{\vee}{f}_{T}(x)=f_{T}(x)\left(1-\chi_{\delta}(x / T)\right)$ tends rapidly to zero with $T \rightarrow \infty$ Her71, He66. In particular, for each natural $N$ and some fixed $N_{0}>4$ there exists a constant $c_{N}$ such that

$$
\int\left|f_{T}(x)\right| d^{4} x \leq c_{N} \frac{s(T)^{N+N_{0}}}{T^{N}} .
$$

We remark that this bound relies on the slow increase of the function $s(T)$, so Condition $A^{\prime}$ cannot be eliminated simply by modifying this function.

As was observed first by Hepp He65, particularly strong estimates on commutators can be obtained in the case of particles moving with different velocities.

Lemma A.2.2. Let $A_{1}\left(f_{1, T}\right), A_{2}\left(f_{2, T}\right), A_{3}\left(f_{3, T}\right)$ be defined as above. Moreover, let $\tilde{f}_{1}$, $\tilde{f}_{2}$ have disjoint velocity supports. Then, for each natural $N$, there exists a constant $c_{N}$ such that:

(a) $\left\|\left[A_{1}\left(f_{1, T}\right), A_{2}\left(f_{2, T}\right)\right]\right\| \leq \frac{c_{N}}{T^{N}}$.

(b) $\left\|\left[A_{1}\left(f_{1, T}\right),\left[A_{2}\left(f_{2, T}\right), A_{3}\left(f_{3, T}\right)\right]\right]\right\| \leq \frac{c_{N}}{T^{N}}$.

The same estimates are valid if some of the operators $A\left(f_{T}\right)$ are replaced by their adjoints or time derivatives.

\section{Proof.}

(a) Making use of the decomposition $f_{T}=\hat{f}_{T}+\stackrel{\vee}{f}_{T}$, we obtain

$$
\begin{aligned}
{\left[A_{1}\left(f_{1, T}\right), A_{2}\left(f_{2, T}\right)\right] } & =\left[A_{1}\left(\hat{f}_{1, T}\right), A_{2}\left(\hat{f}_{2, T}\right)\right]+\left[A_{1}\left(\hat{f}_{1, T}\right), A_{2}\left(\stackrel{\vee}{f}_{2, T}\right)\right] \\
& +\left[A_{1}\left(\stackrel{\vee}{f}_{1, T}\right), A_{2}\left(\hat{f}_{2, T}\right)\right]+\left[A_{1}\left(\stackrel{\vee}{f}_{1, T}\right), A_{2}\left(\stackrel{\vee}{f}_{2, T}\right)\right] .
\end{aligned}
$$


The first term on the r.h.s. is a commutator of two local operators. For sufficiently large $T$ their localization regions become spatially separated because of disjointness of the velocity supports of $\tilde{f}_{1}$ and $\tilde{f}_{2}$. Then the commutator vanishes by virtue of locality. Each of the remaining terms contains a factor $A\left(\stackrel{\vee}{f}{ }_{T}\right)$ which decreases in norm faster than any power of $T^{-1}$ by estimate A.2.7). It is multiplied by $A\left(\hat{f}_{T}\right)$ which increases in norm only as $T^{3 / 2}$ by Lemma A.2.1 (a).

(b) First, let us suppose that $\tilde{f}_{3}$ and $\tilde{f}_{2}$ have disjoint velocity supports. Then $\left[A_{2}\left(f_{2, T}\right), A_{3}\left(f_{3, T}\right)\right]$ decreases fast in norm as a consequence of part (a) of this lemma. Recalling that the norm of $A_{1}\left(f_{1, T}\right)$ increases at most as $T^{3 / 2}$ the assertion follows. Now suppose that $\tilde{f}_{3}$ and $\tilde{f}_{1}$ have disjoint velocity supports. Then, by application of the Jacobi identity, we arrive at the previous situation. In the general case we use a smooth partition of unity to decompose $\tilde{f}_{3}$ into a sum of two functions, each belonging to one of the two special classes studied above.

The statement about adjoints is obvious. To justify the claim concerning derivatives we note that

$$
\frac{d}{d T} A\left(f_{T}\right)=-\frac{1}{s(T)}\left\{\frac{d s(T)}{d T}\left(A\left(f_{T}\right)+A\left(f_{a T}\right)\right)+A\left(f_{b T}\right)\right\}
$$

where $f_{a T}$ is constructed using $h_{a}(t)=t \frac{d h(t)}{d t}$ and $f_{b T}$ contains $h_{b}(t)=\frac{d h(t)}{d t}$. Although $h_{a}$ and $h_{b}$ do not satisfy all the conditions imposed previously on functions $h$, they are elements of $S(\mathbb{R})$. This property suffices to prove the decomposition $f_{T}=\hat{f}_{T}+\stackrel{\vee}{f}_{T}$.

Having constructed creation operators and studied their properties, it will be fairly simple to demonstrate the existence of the asymptotic states. The following theorem uses the original method of Haag [Ha58] modified by Araki $\underline{\mathrm{Ar}}$.

Theorem A.2.3. Suppose that local operators $A_{1}, \ldots, A_{n}$ are regular, $\tilde{f}_{1}, \ldots, \tilde{f}_{n}$ have disjoint velocity supports and vanish sufficiently fast at zero. Moreover, $s(T)=T^{\nu}, \frac{1}{1+\varepsilon}<$ $\nu<1$, where $\varepsilon$ is the exponent appearing in the regularity condition $A^{\prime}$. Let us denote $\Psi(T)=A_{1}\left(f_{1 T}\right) \ldots A_{n}\left(f_{n T}\right) \Omega$. Then there exists the limit $\Psi^{+}=s-\lim _{T \rightarrow \infty} \Psi(T)$ and it is called the asymptotic state.

Proof. We verify the Cauchy condition using Cook's method

$$
\left\|\Psi\left(T_{2}\right)-\Psi\left(T_{1}\right)\right\| \leq \int_{T_{1}}^{T_{2}}\left\|\frac{d \Psi(T)}{d T}\right\| d T .
$$

Now it has to be checked whether the integrand decays sufficiently fast when $T \rightarrow \infty$. By using the Leibniz rule, and then commuting the derivatives of creation operators with the other operators until they act on the vacuum, we arrive at the following expression

$$
\begin{aligned}
\frac{d \Psi}{d T} & =\sum_{k=1}^{n} A_{1}\left(f_{1, T}\right) \ldots \frac{d}{d T} A_{k}\left(f_{k, T}\right) \ldots A_{n}\left(f_{n, T}\right) \Omega \\
& =\sum_{k=1}^{n}\left\{\sum_{l=k+1}^{n} A_{1}\left(f_{1, T}\right) \ldots\left[\frac{d}{d T} A_{k}\left(f_{k, T}\right), A_{l}\left(f_{l, T}\right)\right] \ldots A_{n}\left(f_{n, T}\right) \Omega\right. \\
& \left.+A_{1}\left(f_{1, T}\right) \ldots \check{k} \ldots A_{n}\left(f_{n, T}\right) \frac{d}{d T} A_{k}\left(f_{k, T}\right) \Omega\right\}
\end{aligned}
$$


where $\check{k}$ denotes omission of $A_{k}\left(f_{k T}\right)$. Each term containing commutators vanishes in norm faster than any power of $T^{-1}$ since the rapid decay of commutators, proved in Lemma A.2.2 (a), suppresses the polynomial increase of $\left\|A\left(f_{T}\right)\right\|$ shown in Lemma A.2.1 (a). To estimate the remaining terms we first note that, by virtue of formula A.2.9) and Lemma A.2.1 (b), the vector $\frac{d}{d T} A_{k}\left(f_{k, T}\right) \Omega$ has a compact spectral support $\Delta$. Consequently,

$$
\begin{aligned}
& \left\|A_{1}\left(f_{1, T}\right) \ldots \check{k} \ldots A_{n}\left(f_{n, T}\right) \frac{d}{d T} A_{k}\left(f_{k, T}\right) \Omega\right\| \\
= & \left\|A_{1}\left(f_{1, T}\right) \ldots \check{k} \ldots A_{n}\left(f_{n, T}\right) P(\Delta) \frac{d}{d T} A_{k}\left(f_{k, T}\right) \Omega\right\| \\
\leq & \left\|A_{1}\left(f_{1, T}\right) \ldots \check{k} \ldots A_{n}\left(f_{n, T}\right) P(\Delta)\right\|\left\|\frac{d}{d T} A_{k}\left(f_{k, T}\right) \Omega\right\| \leq \frac{c}{s(T)^{1+\varepsilon}},
\end{aligned}
$$

where in the last step we made use of Lemma A.2.1 (c) and estimate A.1.5). As $\nu(1+\varepsilon)>$ 1 , the integral A.2.10) tends to zero when $T_{1}, T_{2} \rightarrow \infty$ and the Cauchy condition is satisfied.

It is a remarkable feature of the asymptotic states with disjoint velocity supports that already at this stage it is possible to prove that they depend only on the single-particle states $P_{[m]} A(f) \Omega$ rather than on the specific $A, \tilde{f}, h$, and $s$ that were used to construct them. As the possibility to relax the increase of functions $s(T)$ is particularly important for us, we temporarily introduce the notation $A\left(f_{T}^{s}\right)$ to distinguish between operators containing different functions $s(T)$. The following lemma is due to D. Buchholz.

Lemma A.2.4. Suppose that the families of operators $A_{1}\left(f_{1, T}^{s}\right), \ldots, A_{n}\left(f_{n, T}^{s}\right)$, resp. $\widehat{A}_{1}\left(\widehat{f}_{1, T}^{\hat{s}}\right), \ldots, \widehat{A}_{n}\left(\widehat{f}_{n, T}^{\hat{s}}\right)$, satisfy the following conditions:

(a) The functions $\tilde{f}_{1}, \ldots, \tilde{f}_{n}$, resp. $\tilde{\widehat{f}}_{1}, \ldots, \tilde{\widehat{f}}_{n}$, have, within each family, disjoint velocity supports and vanish sufficiently fast at zero.

(b) $P_{[m]} A_{i}\left(f_{i}\right) \Omega=P_{[m]} \widehat{A}_{i}\left(\widehat{f}_{i}\right) \Omega, i \in\{1, \ldots, n\}$, i.e. the single-particle states corresponding to the two families of operators coincide.

(c) $\Psi^{+}=s-\lim _{T \rightarrow \infty} A_{1}\left(f_{1, T}^{s}\right) \ldots A_{n}\left(f_{n, T}^{s}\right) \Omega$ exists.

Then the limit $\widehat{\Psi}^{+}=s-\lim _{T \rightarrow \infty} \widehat{A}_{1}\left(\widehat{f}_{1, T}^{\hat{s}}\right) \ldots \widehat{A}_{n}\left(\widehat{f}_{n, T}^{\hat{s}}\right) \Omega$ exists and coincides with $\Psi^{+}$.

Proof. We proceed by induction. For $n=1$ the assertion is satisfied by assumption. Let us assume that it is satisfied for states involving $n-1$ creation operators. Then the following inequality establishes the strong convergence of the net $A_{1}\left(f_{1, T}^{s}\right) \widehat{A}_{2}\left(\widehat{f}_{2, T}^{s}\right) \ldots \widehat{A}_{n}\left(\widehat{f}_{n, T}^{s}\right) \Omega$

$$
\begin{aligned}
& \left\|A_{1}\left(f_{1, T}^{s}\right) A_{2}\left(f_{2, T}^{s}\right) \ldots A_{n}\left(f_{n, T}^{s}\right) \Omega-A_{1}\left(f_{1, T}^{s}\right) \widehat{A}_{2}\left(\widehat{f}_{2, T}^{\hat{s}}\right) \ldots \widehat{A}_{n}\left(\widehat{f}_{n, T}^{s}\right) \Omega\right\| \\
\leq & \left\|A_{1}\left(f_{1, T}^{s}\right) P(\Delta)\right\|\left\|A_{2}\left(f_{2, T}^{s}\right) \ldots A_{n}\left(f_{n, T}^{s}\right) \Omega-\widehat{A}_{2}\left(\widehat{f}_{2, T}^{s}\right) \ldots \widehat{A}_{n}\left(\widehat{f}_{n, T}^{s}\right) \Omega\right\|,
\end{aligned}
$$

where $\Delta$ is the spectral support of the product of creation operators acting on the vacuum which is compact by Lemma A.2.1 (b). The r.h.s. of this expression vanishes in the limit of large $T$ as a consequence of estimate A.1.8 and the induction hypothesis. By applying the bound on commutators proved in Lemma A.2.2 (a) and the estimate from 
Lemma A.2.1 (a) we verify that also $\widehat{A}_{2}\left(\widehat{f}_{2, T}^{\hat{s}}\right) \ldots \widehat{A}_{n}\left(\widehat{f}_{n, T}^{\hat{s}}\right) A_{1}\left(f_{1, T}^{s}\right) \Omega$ converges strongly and has the same limit. Finally, our claim follows from the estimate

$$
\begin{aligned}
& \left\|\widehat{A}_{2}\left(\widehat{f}_{2, T}^{s}\right) \ldots \widehat{A}_{n}\left(\widehat{f}_{n, T}^{s}\right)\left(\widehat{A}_{1}\left(\widehat{f}_{1, T}^{s}\right)-A_{1}\left(f_{1, T}^{s}\right)\right) \Omega\right\| \\
\leq & \left\|\widehat{A}_{2}\left(\widehat{f}_{2, T}^{s}\right) \ldots \widehat{A}_{n}\left(\widehat{f}_{n, T}^{s}\right) P\left(\Delta_{1}\right)\right\|\left\|\left(\widehat{A}_{1}\left(\widehat{f}_{1, T}^{s}\right)-A_{1}\left(f_{1, T}^{s}\right)\right) \Omega\right\|,
\end{aligned}
$$

where $\Delta_{1}$ is again a compact spectral support. The r.h.s. of this inequality tends to zero with $T \rightarrow \infty$ by assumption (b) and the bound in Lemma A.2.1 (c).

\section{A.3 Fock Structure of Asymptotic States}

It was instrumental in the original proof of the existence of the asymptotic states that $s(T)=T^{\nu}$, where $\nu$ was sufficiently close to one. Lemma A.2.4 allows us to relax this condition and choose any $0<\nu<1$. Using this piece of information, we will verify the Fock structure of the scattering states by the following strategy: First, we establish a counterpart of the relation $a a^{*} \Omega=\left(\Omega\left|a a^{*}\right| \Omega\right) \Omega$ satisfied by the ordinary creation and annihilation operators. Once this equality is proven in the sense of strong limits, we combine it with the double commutator bound from Lemma A.2.2 (b) to obtain the factorization of the scalar product of the scattering states.

We start from two definitions: $\mathcal{O}(r)$ is the double cone of radius $r$. By $\mathfrak{A}_{0}$ we denote the weakly dense subalgebra of $\mathfrak{A}$ consisting of operators for which the operator valued functions $x \rightarrow A(x)$ are infinitely often differentiable in the norm topology. (We remark that, given any regular operator, we can construct a regular operator in $\mathfrak{A}_{0}$ by smearing it with a smooth function).

Now we proceed along the lines set in the scattering theory of massless bosons. (See Bu77. p.169). The analysis is based on the following result, due to Araki, Hepp and Ruelle, on the two-point function of operators from $\mathfrak{A}_{0}$ localized in double cones.

Lemma A.3.1. [AHR62] Let $C_{1}$ and $C_{2}$ be local operators in $\mathfrak{A}_{0}$, localized in double cones $\mathcal{O}\left(r_{1}\right), \mathcal{O}\left(r_{2}\right)$. Then, for all $|\vec{x}| \geq 2\left(r_{1}+r_{2}\right)$,

$$
\left|\left(\Omega \mid C_{1}(\vec{x})\left(1-P_{0}\right) C_{2} \Omega\right)\right| \leq c|\vec{x}|^{-2}\left(r_{1}+r_{2}\right)^{3}\left\{\left\|C_{1}^{*} \Omega\right\|\left\|\partial_{0} C_{2} \Omega\right\|+\left\|C_{2}^{*} \Omega\right\|\left\|\partial_{0} C_{1} \Omega\right\|\right\},
$$

where $\left.P_{0}=\mid \Omega\right)\left(\Omega \mid\right.$ and $c$ is a constant which depends neither on $\vec{x}$ nor on $C_{1}, C_{2}$ and $r_{1}, r_{2}$.

We will apply this lemma, taking as $C_{1}$ and $C_{2}$ the commutators $\left[A_{i}\left(t_{i}, \vec{x}_{i}\right), A_{j}\left(t_{j}, \vec{x}_{j}\right)\right]$. It will be used in the proof of the following statement that these operators are localized in regions of finite volume, as long as the differences $\left|t_{i}-t_{j}\right|$ are kept small.

Lemma A.3.2. Let $A_{1}, \ldots, A_{4} \in \mathfrak{A}_{0}$ be localized in double cones $\mathcal{O}\left(r_{1}\right), \ldots, \mathcal{O}\left(r_{4}\right)$. We define

$$
K=\left(\Omega \mid\left[A_{1}\left(t_{1}, \vec{x}_{1}\right), A_{2}\left(t_{2}, \vec{x}_{2}\right)\right]\left(1-P_{0}\right)\left[A_{3}\left(t_{3}, \vec{x}_{3}\right), A_{4}\left(t_{4}, \vec{x}_{4}\right)\right] \Omega\right),
$$

where $\left.P_{0}=\mid \Omega\right)(\Omega \mid$. Then the following estimate holds

$$
|K| \leq c \chi\left(\left|\vec{x}_{1}-\vec{x}_{2}\right| \leq R\right) \chi\left(\left|\vec{x}_{3}-\vec{x}_{4}\right| \leq R\right) \cdot\left\{\begin{array}{cl}
1 & \text { if }\left|\vec{x}_{2}-\vec{x}_{3}\right| \leq 4 R \\
\frac{R^{3}}{\vec{x}_{2}-\left.\vec{x}_{3}\right|^{2}+R^{2}} & \text { if }\left|\vec{x}_{2}-\vec{x}_{3}\right|>4 R
\end{array}\right.
$$


where $R=\sum_{i=1}^{4}\left(r_{i}+\left|t-t_{i}\right|\right), t=\frac{1}{4}\left(t_{1}+t_{2}+t_{3}+t_{4}\right)$ and $\chi$ are the characteristic functions of the respective sets. The constant $c$ depends neither on $t_{1}, \ldots, t_{4}$ nor on $r_{1}, \ldots, r_{4}$.

Proof. Using the translation invariance of the vacuum, we can rewrite $K$ as follows:

$$
K=\left(\Omega \mid\left[A_{1}\left(t_{1}-t, \vec{r}_{12}\right), A_{2}\left(t_{2}-t, \vec{r}_{21}\right)\right] U(\vec{x})\left(1-P_{0}\right)\left[A_{3}\left(t_{3}-t, \vec{r}_{34}\right), A_{4}\left(t_{4}-t, \vec{r}_{43}\right)\right] \Omega\right),
$$

where $\vec{r}_{i k}=\frac{1}{2}\left(\vec{x}_{i}-\vec{x}_{k}\right), \vec{x}=\frac{1}{2}\left(\vec{x}_{1}+\vec{x}_{2}-\vec{x}_{3}-\vec{x}_{4}\right)$. The commutator $\left[A_{i}\left(t_{i}-t, \vec{r}_{i k}\right), A_{k}\left(t_{k}-\right.\right.$ $\left.\left.t, \vec{r}_{k i}\right)\right]$ is localized in a double cone of radius $r$ given by

$$
r=\left|\vec{r}_{i k}\right|+r_{i}+r_{k}+\left|t_{i}-t\right|+\left|t_{k}-t\right| .
$$

But it is zero (owing to locality) if

$$
2\left|\vec{r}_{i k}\right| \geq r_{i}+r_{k}+\left|t_{i}-t\right|+\left|t_{k}-t\right| \text {. }
$$

Consequently, there follows the bound for $r$

$$
r \leq \frac{3}{2}\left(r_{i}+r_{k}+\left|t_{i}-t\right|+\left|t_{k}-t\right|\right) .
$$

Now we can apply Lemma A.3.1 and get for $|\vec{x}| \geq 3 \sum_{i=1}^{4}\left(r_{i}+\left|t_{i}-t\right|\right)$

$$
\begin{aligned}
|K| \leq & c|\vec{x}|^{-2}\left(\sum_{i=1}^{4}\left(r_{i}+\left|t_{i}-t\right|\right)\right)^{3} \\
\cdot & \left\{\left\|\left[A_{1}\left(t_{1}, \vec{x}_{1}\right), A_{2}\left(t_{2}, \vec{x}_{2}\right)\right]^{*} \Omega\right\| \|\left[\partial_{0} A_{3}\left(t_{3}, \vec{x}_{3}\right), A_{4}\left(t_{4}, \vec{x}_{4}\right)\right] \Omega\right. \\
+ & {\left.\left[A_{3}\left(t_{3}, \vec{x}_{3}\right), \partial_{0} A_{4}\left(t_{4}, \vec{x}_{4}\right)\right] \Omega \|+(1 \leftrightarrow 3,2 \leftrightarrow 4)\right\} . }
\end{aligned}
$$

The r.h.s. of this expression vanishes, if either of the following two conditions holds

$$
\begin{aligned}
& \left|\vec{x}_{1}-\vec{x}_{2}\right| \geq\left|t_{1}-t_{2}\right|+r_{1}+r_{2}, \\
& \left|\vec{x}_{3}-\vec{x}_{4}\right| \geq\left|t_{3}-t_{4}\right|+r_{3}+r_{4} .
\end{aligned}
$$

We may therefore estimate $|\vec{x}|$ as follows

$$
\begin{aligned}
|\vec{x}| & =\frac{1}{2}\left|2\left(\vec{x}_{2}-\vec{x}_{3}\right)-\left(\vec{x}_{2}-\vec{x}_{1}\right)-\left(\vec{x}_{4}-\vec{x}_{3}\right)\right| \\
& \geq\left|\vec{x}_{2}-\vec{x}_{3}\right|-\frac{1}{2}\left(\left|\vec{x}_{2}-\vec{x}_{1}\right|+\left|\vec{x}_{4}-\vec{x}_{3}\right|\right) \\
& \geq\left|\vec{x}_{2}-\vec{x}_{3}\right|-\frac{1}{2} \sum_{i=1}^{4}\left(r_{i}+\left|t_{i}-t\right|\right)=\left|\vec{x}_{2}-\vec{x}_{3}\right|-\frac{1}{2} R .
\end{aligned}
$$

If $\left|\vec{x}_{2}-\vec{x}_{3}\right|>4 R$, then $|\vec{x}| \geq 3 R$ and it follows from the bound A.3.8 that

$$
|K| \leq c R^{3}\left(\left|\vec{x}_{2}-\vec{x}_{3}\right|^{2}+R^{2}\right)^{-1} \chi\left(\left|\vec{x}_{1}-\vec{x}_{2}\right| \leq R\right) \chi\left(\left|\vec{x}_{3}-\vec{x}_{4}\right| \leq R\right) .
$$

When $\left|\vec{x}_{2}-\vec{x}_{3}\right| \leq 4 R$, we estimate trivially

$$
\begin{aligned}
|K| & \leq\left\|\left[A_{1}\left(t_{1}, \vec{x}_{1}\right), A_{2}\left(t_{2}, \vec{x}_{2}\right)\right]\right\|\left\|\left[A_{3}\left(t_{3}, \vec{x}_{3}\right), A_{4}\left(t_{4}, \vec{x}_{4}\right)\right]\right\| \\
& \leq c^{\prime} \chi\left(\left|\vec{x}_{1}-\vec{x}_{2}\right| \leq R\right) \chi\left(\left|\vec{x}_{3}-\vec{x}_{4}\right| \leq R\right),
\end{aligned}
$$

what concludes the proof.

Now we are ready to prove that the product of a creation operator and an annihilation operator acting on the vacuum reproduces it. 
Proposition A.3.3. Suppose that $A_{1}, A_{2}$ are local operators from $\mathfrak{A}_{0}, s(T)=T^{\nu}, \nu<$ 1/8. Then

$$
s-\lim _{T \rightarrow \infty} A_{1}\left(f_{1}\right)^{*} A_{2}\left(f_{2}\right) \Omega=\left(\Omega \mid A_{1}\left(f_{1}\right)^{*} P_{[m]} A_{2}\left(f_{2}\right) \Omega\right) \Omega .
$$

Proof. We start by performing the integration of the function $K$ from the preceding lemma with the regular Klein-Gordon wave-packets and estimating the behavior of the resulting function of $t_{1}, \ldots, t_{4}$. We will change the variables of integration to $\vec{u}_{1}=\vec{x}_{2}-\vec{x}_{1}$, $\vec{u}_{2}=\vec{x}_{2}, \vec{u}_{3}=\vec{x}_{3}-\vec{x}_{2}, \vec{u}_{4}=\vec{x}_{4}-\vec{x}_{3}$. In the region $\left|\vec{x}_{2}-\vec{x}_{3}\right|>4 R$ we obtain

$$
\begin{aligned}
& \int d^{3} x_{1}\left|f_{1}\left(t_{1}, \vec{x}_{1}\right)\right| \ldots \int d^{3} x_{4}\left|f_{4}\left(t_{4}, \vec{x}_{4}\right)\right||K| \\
\leq & c R^{9}\left(1+\left|t_{1}\right|\right)^{-3 / 2}\left(1+\left|t_{4}\right|\right)^{-3 / 2} \int d^{3} u_{2} \int d^{3} u_{3} \frac{\left|f_{2}\left(t_{2}, \vec{u}_{2}\right)\right|\left|f_{3}\left(t_{3}, \vec{u}_{3}+\vec{u}_{2}\right)\right|}{\left|\vec{u}_{3}\right|^{2}+R^{2}} \\
\leq & c R^{9}\left(1+\left|t_{1}\right|\right)^{-3 / 2}\left(1+\left|t_{4}\right|\right)^{-3 / 2}\left(1+\left|t_{2}\right|\right)^{3 / 2} .
\end{aligned}
$$

Here in the first step we applied the bound A.2.1 to $f_{1}$ and $f_{4}$. In the second step we exploited estimate A.2.2 to control the integral w.r.t. $\vec{u}_{3}$ and we used the inequality $2 \frac{\left|f_{3}(\cdot, \cdot)\right|}{\left|\vec{u}_{3}\right|^{2}+R^{2}} \leq\left(\frac{1}{\left|\vec{u}_{3}\right|^{2}+R^{2}}\right)^{2}+\left|f_{3}(\cdot, \cdot)\right|^{2}$ in order to verify that the integral over $\vec{u}_{3}$ is bounded in $t_{3}$. In the region $\left|\vec{x}_{2}-\vec{x}_{3}\right| \leq 4 R$ our estimate becomes improved by the factor $\left(1+\left|t_{3}\right|\right)^{-3 / 2}$, so A.3.15 holds without restrictions.

Since $A\left(f_{T}\right)^{*} \Omega=0$ for sufficiently large $T$, we can estimate

$$
\begin{aligned}
& \left\|A_{1}\left(f_{1, T}\right)^{*} A_{2}\left(f_{2, T}\right) \Omega-\left(\Omega \mid A_{1}\left(f_{1, T}\right)^{*} A_{2}\left(f_{2, T}\right) \Omega\right) \Omega\right\|^{2} \\
= & \left(\Omega \mid\left[A_{2}\left(f_{2, T}\right)^{*}, A_{1}\left(f_{1, T}\right)\right]\left(1-P_{0}\right)\left[A_{1}\left(f_{1, T}\right)^{*}, A_{2}\left(f_{2, T}\right)\right] \Omega\right) \\
\leq & \int d t_{1} h_{T}\left(t_{1}\right) \ldots \int d t_{4} h_{T}\left(t_{4}\right) c R^{9}\left(1+\left|t_{1}\right|\right)^{-3 / 2}\left(1+\left|t_{4}\right|\right)^{-3 / 2}\left(1+\left|t_{2}\right|\right)^{3 / 2} \\
\leq & C \frac{s(T)^{12}}{T^{3 / 2}}
\end{aligned}
$$

Now the assertion follows from the slow increase of the function $s(T)$.

After this preparation it is straightforward to calculate the scalar product of two asymptotic states.

Theorem A.3.4. Suppose that $A_{1}\left(f_{1, T}\right), \ldots, A_{n}\left(f_{n, T}\right)$, resp. $\widehat{A}_{1}\left(\widehat{f}_{1, T}\right), \ldots, \widehat{A}_{n}\left(\widehat{f}_{n, T}\right)$, are two families of creation operators constructed using local operators from $\mathfrak{A}_{0}$, functions $\tilde{f}_{i}$, resp. $\tilde{\widehat{f}}_{i}, i=1, \ldots, n$, vanishing sufficiently fast at zero and having, within each family, disjoint velocity supports. Moreover, $s(T)=T^{\nu}$, where $0<\nu<1$. Then

$$
\begin{array}{r}
\lim _{T \rightarrow \infty}\left(\Omega \mid A_{n}\left(f_{n, T}\right)^{*} \ldots A_{1}\left(f_{1, T}\right)^{*} \widehat{A}_{1}\left(\widehat{f}_{1, T}\right) \ldots \widehat{A}_{n}\left(\widehat{f}_{n, T}\right) \Omega\right) \\
=\sum_{\sigma \in S_{n}}\left(\Omega \mid A_{1}\left(f_{1}\right)^{*} P_{[m]} \widehat{A}_{\sigma_{1}}\left(\widehat{f}_{\sigma_{1}}\right) \Omega\right) \ldots\left(\Omega \mid A_{n}\left(f_{n}\right)^{*} P_{[m]} \widehat{A}_{\sigma_{n}}\left(\widehat{f}_{\sigma_{n}}\right) \Omega\right) .
\end{array}
$$

Here the sum is over all permutations of an n-element set.

Proof. First, we make use of Lemma A.2.4 to ensure a sufficiently slow increase of the function $s(T)$. Next, we proceed by induction. For $n=1$ the theorem is trivially true. 
Let us assume that it is true for $n-1$ and compute

$$
\begin{aligned}
& \left(\Omega \mid A_{n}\left(f_{n, T}\right)^{*} \ldots A_{1}\left(f_{1, T}\right)^{*} \widehat{A}_{1}\left(\widehat{f}_{1, T}\right) \ldots \widehat{A}_{n}\left(\widehat{f}_{n, T}\right) \Omega\right) \\
= & \sum_{k=1}^{n}\left(\Omega \mid A_{n}\left(f_{n, T}\right)^{*} \ldots A_{2}\left(f_{2, T}\right)^{*} \widehat{A}_{1}\left(\widehat{f}_{1, T}\right) \ldots\left[A_{1}\left(f_{1, T}\right)^{*}, \widehat{A}_{k}\left(\widehat{f}_{k, T}\right)\right] \ldots \widehat{A}_{n}\left(\widehat{f}_{n, T}\right) \Omega\right) \\
= & \sum_{k=1}^{n}\left\{\sum _ { l = k + 1 } ^ { n } \left(\Omega \mid A_{n}\left(f_{n, T}\right)^{*} \ldots A_{2}\left(f_{2, T}\right)^{*} \widehat{A}_{1}\left(\widehat{f}_{1, T}\right) \ldots\right.\right. \\
\ldots & {\left.\left[\left[A_{1}\left(f_{1, T}\right)^{*}, \widehat{A}_{k}\left(\widehat{f}_{k, T}\right)\right], \widehat{A}_{l}\left(\widehat{f}_{l, T}\right)\right] \ldots \widehat{A}_{n}\left(\widehat{f}_{n, T}\right) \Omega\right) } \\
+ & \left.\left(\Omega \mid A_{n}\left(f_{n, T}\right)^{*} \ldots A_{2}\left(f_{2, T}\right)^{*} \widehat{A}_{1}\left(\widehat{f}_{1, T}\right) \ldots \hat{k} \ldots \widehat{A}_{n}\left(\widehat{f}_{n, T}\right) A_{1}\left(f_{1, T}\right)^{*} \widehat{A}_{k}\left(\widehat{f}_{k, T}\right) \Omega\right)\right\} .(\mathrm{A} .
\end{aligned}
$$

The terms containing double commutators vanish in the limit by Lemma A.2.2 (b) and Lemma A.2.1 (a). The remaining terms factorize by the preceding proposition and by Lemma A.2.1 (b) and (c):

$$
\begin{aligned}
& \lim _{T \rightarrow \infty}\left(\Omega \mid A_{n}\left(f_{n, T}\right)^{*} \ldots A_{2}\left(f_{2, T}\right)^{*} \widehat{A}_{1}\left(\widehat{f}_{1, T}\right) \ldots \check{k} \ldots \widehat{A}_{n}\left(\widehat{f}_{n, T}\right) A_{1}\left(f_{1, T}\right)^{*} \widehat{A}_{k}\left(\widehat{f}_{k, T}\right) \Omega\right) \\
&= \lim _{T \rightarrow \infty}\left(\Omega\left|A_{n}\left(f_{n, T}\right)^{*} \ldots A_{2}\left(f_{2, T}\right)^{*} \widehat{A}_{1}\left(\widehat{f}_{1, T}\right) \ldots \check{k} \ldots \widehat{A}_{n}\left(\widehat{f}_{n, T}\right)\right| \Omega\right) \cdot \\
& \cdot\left(\Omega \mid A_{1}\left(f_{1}\right)^{*} P_{[m]} \widehat{A}_{k}\left(\widehat{f}_{k}\right) \Omega\right) . \quad(\text { A.3. }
\end{aligned}
$$

This quantity factorizes into two-point functions by the induction hypothesis.

It is also evident from the proof that the scalar product of two asymptotic states involving different numbers of operators is zero. Now the Fock structure of the asymptotic states and the construction of the wave operators follows by standard density arguments: By Condition $A^{\prime}$, there exists a regular operator $A \in \mathfrak{A}(\mathcal{O})$ s.t. $P_{[m]} A \Omega \neq 0$. Since the representation $U$ acts irreducibly in $\mathcal{H}^{[m]}$, the vectors $\left\{U(x, \Lambda) P_{[m]} A \Omega \mid(x, \Lambda) \in P_{+}^{\uparrow}\right\}$ span a dense set in this subspace. Making use of the fact that a Poincaré transformation of a regular operator is regular, we obtain that the set of vectors

$$
\left\{P_{[m]} A(f) \Omega \mid A \text {-regular, } \tilde{f} \in C_{0}^{\infty}\left(\mathbb{R}^{3}\right) \text { vanishes sufficiently fast at } 0\right\}
$$

is total in $\mathcal{H}^{[m]}$. In view of Theorem A.2.3 the wave operator $W^{+}$can be defined on a dense set in $\Gamma\left(\mathcal{H}^{[m]}\right)$, extending by linearity the following relation

$$
W^{+}\left(a^{*}\left(P_{[m]} A_{1}\left(f_{1}\right) \Omega\right) \ldots a^{*}\left(P_{[m]} A_{n}\left(f_{n}\right) \Omega\right) \Omega\right)=\mathrm{s}-\lim _{T \rightarrow \infty} A_{1}\left(f_{1, T}\right) \ldots A_{n}\left(f_{n, T}\right) \Omega,
$$

where $A$ and $f$ satisfy the conditions stated in A.3.20). Due to Theorem A.3.4 $W^{+}$ preserves norms, thus it extends to an isometry from $\Gamma\left(\mathcal{H}^{[m]}\right)$ to $\mathcal{H}$. The wave operator $W^{-}$is constructed analogously, making use of the incoming states, and the scattering matrix $S: \Gamma\left(\mathcal{H}^{[m]}\right) \rightarrow \Gamma\left(\mathcal{H}^{[m]}\right)$ is given by $S=\left(W^{+}\right)^{*} W^{-}$.

\section{A.4 Conclusion}

We have constructed a scattering theory of massive particles without the lower and upper mass gap assumptions. The Lorentz covariance of the construction can be verified by application of standard arguments $\mathrm{Ar}$. Including fermions would cause no additional difficulty, as the fermionic creation operators are bounded uniformly in time [Bu75]. 
The only remaining restriction is the regularity assumption $A^{\prime}$. We note that it was used only to establish the existence of the scattering states - the construction of the Fock structure was independent of this property. Moreover, we would like to point out that it does not seem possible to derive it from general postulates. In fact, let us consider the generalized free field $\phi$ with the commutator fixed by the measure $\sigma$ :

$$
[\phi(x), \phi(y)]=\int d \sigma(\lambda) \Delta_{\lambda}(x-y)
$$

where $\Delta_{\lambda}$ is the commutator function of the free field of mass $\sqrt{\lambda}$. Suppose that the measure $\sigma$ contains a discrete mass $m$ and in its neighborhood is defined by the function $F(\lambda)=1 / \ln \left|\lambda-m^{2}\right|$. Then it is easy to find polynomials in the fields smeared with Schwartz class functions which violate the bound from Condition $A^{\prime}$. However, the existence of the scattering states, constructed with the help of such polynomials, can easily be verified using properties of generalized free fields. These observations indicate that Condition $A^{\prime}$ is only of technical nature. To relax it one should probably look for a construction of asymptotic states which avoids Cook's method - perhaps similarly to the scattering theory of massless particles [Bu75, Bu77]. 


\section{Appendix B}

\section{Scalar Free Field Theory and its Phase Space Structure}

In this appendix we collect some known results on the phase space structure of scalar free field theory in a slightly modified form, suitable for our purposes. They provide a basis for the proofs that the new Conditions $L^{(2)}, L^{(1)}, N_{\natural}$ and $C_{\mathrm{b}}$, introduced in the main body of this work, hold in the model of scalar, non-interacting particles. These arguments are given in the subsequent appendices. For Conditions $L^{(1)}$ and $C_{\mathbf{b}}$ only the massive case will be considered.

Verification of phase space conditions in models is an integral part of phase space analysis in QFT as it proves consistency of the introduced criteria with the basic postulates of quantum field theory. This issue was treated already in the seminal paper of Haag and Swieca HS65, who verified their compactness condition in massive scalar free field theory. Serious technical improvements, including the reduction of the problem to a singleparticle question, appeared in the work of Buchholz and Wichmann BWi86, who noted the importance of nuclearity. The massless case was included for the first time in BJa87. Conditions $C_{\sharp}$ and $N_{\sharp}$, stated in Sections 3.1 and 3.5. respectively, were first verified in BP90. A particularly flexible and explicit formulation of the subject was given by Bostelmann in [Bos00] and our presentation relies primarily on this work.

The goal is to construct an expansion of the map $\Theta_{E}: \mathfrak{A}(\mathcal{O}(r)) \rightarrow B(\mathcal{H})$, given by $\Theta_{E}(A)=P_{E} A P_{E}$, into rank-one mappings. More precisely, we are looking for functionals $\tau_{i} \in \mathfrak{A}(\mathcal{O}(r))^{*}$ and operators $S_{i} \in B(\mathcal{H}), i \in \mathbb{N}$, s.t.

$$
\begin{array}{r}
\Theta_{E}(A)=\sum_{i} \tau_{i}(A) S_{i}, \quad A \in \mathfrak{A}(\mathcal{O}(r)), \\
\sum_{i}\left\|\tau_{i}\right\|^{p}\left\|S_{i}\right\|^{p}<\infty, \quad 0<p \leq 1 .
\end{array}
$$

Thus, in view of Lemma 3.5.1, we corroborate the well known fact that Condition $N_{\sharp}$ holds in scalar free field theory BP90. Moreover, we establish properties of the functionals $\left\{\tau_{i}\right\}_{1}^{\infty}$ and $\left\{S_{i}\right\}_{1}^{\infty}$ which will be needed to verify the new conditions introduced in this Thesis.

This appendix is organized as follows: In Section B.1 we explain our multiindex notation. Section B.2 introduces scalar free field theory, its even part and its sub-theory generated by the derivatives of the field. In Section B.3 we construct the functionals 
$\left\{\tau_{i}\right\}_{1}^{\infty}$ and estimate their norms. Apart from the material familiar from Section 7.2.B of Bos00, we establish certain energy bounds on these functionals which we need to verify Condition $L^{(1)}$. In Section B.4 two expansions of the single-particle wavefunctions are developed, following Sections 7.2.2 and 7.2.3 of Bos00. They give rise to an expansion of the map $\Theta_{E}$ into rank-one mappings, which we introduce in Section B.5 It is a variant of the 'mixed expansion' from Section 7.2.6 of [Bos00]. In order to avoid the unduly complicated infrared structure and phase space properties of low dimensional massless models, in this appendix we adopt the following:

Standing assumption: Unless stated otherwise, all statements concerning scalar free field theory hold either for $m>0$ and $s \geq 1$ or $m=0$ and $s \geq 3$.

\section{B.1 Multiindex Notation}

A multiindex is a sequence $\mu=\{\mu(i)\}_{1}^{\infty}$ of elements from $\mathbb{N}_{0}$ s.t. only a finite number of components is different from zero. Addition of multiindices is performed component-wise. The length of a multiindex is given by

$$
|\mu|=\sum_{i} \mu(i)
$$

The factorial of a multiindex is defined as $\mu !=\prod_{i} \mu(i)$ !. Given a sequence $a=\left\{a_{i}\right\}_{1}^{\infty}$, valued in any set with multiplication, its multiindex power is defined as

$$
a^{\mu}=\prod_{i} a_{i}^{\mu(i)}
$$

It is convenient to extend the above conventions to pairs of multiindices $\bar{\mu}=\left(\mu^{+}, \mu^{-}\right)$: The length of such 2-multiindex is given by $|\bar{\mu}|=\left|\mu^{+}\right|+\left|\mu^{-}\right|$and the factorial is defined as $\bar{\mu} !=\mu^{+} ! \mu^{-}$!. Given any sequence of pairs $b=\left\{b_{i}^{+}, b_{i}^{-}\right\}_{1}^{\infty}$, we define the 2-multiindex power of $b$ as follows

$$
b^{\bar{\mu}}=\left(b^{+}\right)^{\mu^{+}}\left(b^{-}\right)^{\mu^{-}} .
$$

Finally, an $n$-index $\kappa$ is a multiindex s.t. $\kappa(i)=0$ for $i>n$. All the above conventions extend naturally to $n$-indices.

We recall that for any sequence of complex numbers $\left\{t_{j}\right\}_{1}^{\infty}$ and $k \in \mathbb{N}$ there holds the multinomial formula

$$
\left(\sum_{j=1}^{n} t_{j}\right)^{k}=\sum_{\mu,|\mu|=k} \frac{|\mu|}{\mu !} t^{\mu},
$$

where the sum on the r.h.s. extends over all $n$-indices of length $k$. Assuming that the sequence $\left\{t_{j}\right\}_{1}^{\infty}$ is absolutely summable, we can take the limit $n \rightarrow \infty$, obtaining on the r.h.s. the sum over all multiindices $\mu$ of length $k$.

\section{B.2 Scalar Free Field Theory and Related Models}

In this section we recall from Section X.7 of [RS2] some basic properties of scalar free field theory of mass $m \geq 0$ in $s$ space dimensions. The single-particle space of this theory is $L^{2}\left(\mathbb{R}^{s}, d^{s} p\right)$. On this space there act the multiplication operators $\omega(\vec{p})=\sqrt{|\vec{p}|^{2}+m^{2}}$ and 
$p_{1}, \ldots, p_{s}$ which are self-adjoint on a suitable dense domain. The unitary representation of the Poincaré group $P_{+}^{\uparrow} \ni(x, \Lambda) \rightarrow U_{1}(x, \Lambda)$, acting on the single-particle space, is given by

$$
\left(U_{1}(x, \Lambda) f\right)(\vec{p})=e^{i\left(\omega(\vec{p}) x^{0}-\vec{p} \vec{x}\right)} f\left(\Lambda^{-1} \vec{p}\right), \quad f \in L^{2}\left(\mathbb{R}^{s}, d^{s} p\right),
$$

where $\Lambda^{-1} \vec{p}$ is the spatial part of the four-vector $\Lambda^{-1}(\omega(\vec{p}), \vec{p})$. The full Hilbert space $\mathcal{H}$ of the theory is the symmetric Fock space over $L^{2}\left(\mathbb{R}^{s}, d^{s} p\right)$. By the method of second quantization, we obtain the unitary representation of the Poincaré group $U(x, \Lambda)=\Gamma\left(U_{1}(x, \Lambda)\right)$ which implements the corresponding family of automorphisms acting on $B(\mathcal{H})$

$$
\alpha_{(x, \Lambda)}(\cdot)=U(x, \Lambda) \cdot U(x, \Lambda)^{*} .
$$

The Hamiltonian $H=d \Gamma(\omega)$, and the momentum operators $P_{i}=d \Gamma\left(p_{i}\right), i=1,2, \ldots, s$ are defined on a suitable domain in $\mathcal{H}$. The joint spectrum of this family of commuting, self-adjoint operators is contained in the closed forward light cone.

The elements of $\mathcal{H}$ have the form $\Psi=\left\{\Psi_{n}\right\}_{n=0}^{\infty}$, where $\Psi_{n}$ is an $n$-particle vector. We denote by $D_{F}$ the dense subspace of finite particle vectors, consisting of such $\Psi$ that $\Psi_{n} \neq 0$ only for finitely many $n$. On this subspace we define the annihilation operator $a(f), f \in L^{2}\left(\mathbb{R}^{s}, d^{s} p\right)$, given by the formula

$$
(a(f) \Psi)_{n}\left(\vec{p}_{1}, \ldots, \vec{p}_{n}\right)=\sqrt{n+1} \int d^{s} p \bar{f}(\vec{p}) \Psi_{n+1}\left(\vec{p}, \vec{p}_{1}, \ldots, \vec{p}_{n}\right)
$$

and its adjoint $a^{*}(f)$. With the help of these operators we construct the (time zero) canonical fields and momenta for any $g \in S\left(\mathbb{R}^{s}\right)$

$$
\begin{aligned}
& \phi_{+}(g)=\frac{1}{\sqrt{2}}\left(a^{*}\left(\omega^{-\frac{1}{2}} \tilde{g}\right)+a\left(\omega^{-\frac{1}{2}} \tilde{\bar{g}}\right)\right), \\
& \phi_{-}(g)=\frac{1}{\sqrt{2}}\left(a^{*}\left(i \omega^{\frac{1}{2}} \tilde{g}\right)+a\left(i \omega^{\frac{1}{2}} \tilde{\bar{g}}\right)\right) .
\end{aligned}
$$

The algebra $\mathfrak{A}(\mathcal{O}(r))$ of observables localized in the double cone $\mathcal{O}(r)$, whose base is the $s$-dimensional ball $\mathcal{O}_{r}$ of radius $r$, is given by

$$
\mathfrak{A}(\mathcal{O}(r))=\left\{e^{i \sqrt{2} \phi_{+}\left(F^{+}\right)+i \sqrt{2} \phi_{-}\left(F^{-}\right)} \mid F^{ \pm} \in D\left(\mathcal{O}_{r}\right)_{\mathbb{R}}\right\}^{\prime \prime},
$$

where $D\left(\mathcal{O}_{r}\right)_{\mathbb{R}}$ is the space of real-valued test functions supported in this ball. Let us mention an equivalent definition of the local algebra which is more convenient for some purposes: We define the following (non-closed) subspaces in $L^{2}\left(\mathbb{R}^{s}, d^{s} p\right)$

$$
\stackrel{\circ}{\mathcal{L}}_{r}^{ \pm}=\omega^{\mp \frac{1}{2}} \widetilde{D}\left(\mathcal{O}_{r}\right),
$$

where tilde denotes the Fourier transform. We denote by $J$ the complex conjugation in configuration space and set

$$
\stackrel{\circ}{\mathcal{L}}_{r}=(1+J) \stackrel{\circ}{\mathcal{L}}_{r}^{+}+(1-J) \stackrel{\circ}{\mathcal{L}}_{r}^{-} .
$$

Then every $f \in \stackrel{\circ}{\mathcal{L}}_{r}$ has the form $f=f^{+}+i f^{-}$, where

$$
\tilde{f}^{ \pm}=\omega^{\mp \frac{1}{2}} \tilde{F}^{ \pm}
$$


for some $F^{ \pm} \in D\left(\mathcal{O}_{r}\right)_{\mathbb{R}}$. For any $f \in \stackrel{\circ}{\mathcal{L}}_{r}$ we define the Weyl operator

$$
W(f):=e^{i\left(a^{*}(f)+a(f)\right)}=e^{i \sqrt{2} \phi_{+}\left(F^{+}\right)+i \sqrt{2} \phi_{-}\left(F^{-}\right)} .
$$

In view of the second equality and definition (B.2.6) there holds

$$
\mathfrak{A}(\mathcal{O}(r))=\left\{W(f) \mid f \in \stackrel{\circ}{\mathcal{L}}_{r}\right\}^{\prime \prime} .
$$

With the help of the translation automorphisms $\alpha_{x}$, introduced above, we define local algebras attached to double cones centered at any point $x$ of spacetime

$$
\mathfrak{A}(\mathcal{O}(r)+x)=\alpha_{x}(\mathfrak{A}(\mathcal{O}(r))) .
$$

Now for any open, bounded region $\mathcal{O}$ we set

$$
\mathfrak{A}(\mathcal{O})=\left(\bigcup_{\substack{r, x \\ \mathcal{O}(r)+x \subset \mathcal{O}}} \mathfrak{A}(\mathcal{O}(r)+x)\right)^{\prime \prime},
$$

obtaining the local net $\mathfrak{A}$. The global algebra, denoted by the same symbol, is the $C^{*}$ inductive limit of all such local algebras. It is well known that the triple $(\mathfrak{A}, \alpha, \mathcal{H})$ satisfies the postulates 1-5 from Section 1.6 [RS2].

We can immediately construct two related theories: First, we define the local algebra generated by the derivatives of the free field

$$
\mathfrak{A}^{(\mathrm{d})}(\mathcal{O}(r))=\left\{e^{i \sqrt{2} \phi_{+}\left(\sum_{j=1}^{s} \partial_{x_{j}} F_{j}^{+}\right)+i \sqrt{2} \phi_{-}\left(F^{-}\right)} \mid F_{j}^{+}, F^{-} \in D\left(\mathcal{O}_{r}\right)_{\mathbb{R}}, j \in\{1, \ldots, s\}\right\}^{\prime \prime},
$$

which is clearly a subalgebra of $\mathfrak{A}(\mathcal{O}(r))$. In the massive case one can show that $\mathfrak{A}^{(\mathrm{d})}(\mathcal{O}(r))=\mathfrak{A}(\mathcal{O}(r))$, making use of the equation of motion. In the massless case, however, the inclusion is proper. The global $C^{*}$-algebra, constructed as in the case of the full theory, is denoted by $\mathfrak{A}^{(\mathrm{d})}$ and the theory $\left(\mathfrak{A}^{(\mathrm{d})}, \alpha, \mathcal{H}\right)$ satisfies the postulates from Section 1.6

The second example is the even part of scalar free field theory. Here the local algebra, attached to the double cone $\mathcal{O}(r)$, is given by

$$
\mathfrak{A}^{(\mathrm{e})}(\mathcal{O}(r))=\left\{\cos \left(\sqrt{2} \phi_{+}\left(F^{+}\right)+\sqrt{2} \phi_{-}\left(F^{-}\right)\right) \mid F^{+}, F^{-} \in D\left(\mathcal{O}_{r}\right) \mathbb{R}^{\prime \prime} .\right.
$$

The corresponding local net $\mathfrak{A}^{(\mathrm{e})}$, constructed as above, gives rise to the theory $\left(\mathfrak{A}^{(\mathrm{e})}, \alpha, \mathcal{H}\right)$ which satisfies all the postulates from Section [1.6 except for irreducibility. In order to ensure this latter property, we represent the algebra $\mathfrak{A}^{(\mathrm{e})}$ on the subspace $\mathcal{H}^{(\mathrm{e})}$ in $\mathcal{H}$ spanned by vectors with even particle numbers. We define $\underline{\mathfrak{A}}^{(\mathrm{e})}=\left.\mathfrak{A}^{(\mathrm{e})}\right|_{\mathcal{H}^{(\mathrm{e})}}, \underline{U}(x, \Lambda)=\left.U(x, \Lambda)\right|_{\mathcal{H}^{(\mathrm{e})}}$ and $\underline{\alpha}_{(x, \Lambda)}(\cdot)=\underline{U}(x, \Lambda) \cdot \underline{U}(x, \Lambda)^{-1}$. The resulting theory $\left(\underline{\mathfrak{A}}^{(\mathrm{e})}, \underline{\alpha}, \mathcal{H}^{(\mathrm{e})}\right)$ satisfies all the general postulates.

For future convenience we discuss the relation between the theory $\left(\mathfrak{A}^{(\mathrm{e})}, \alpha, \mathcal{H}\right)$, acting on the full Fock space, and the theory $\left(\underline{\mathfrak{A}}^{(\mathrm{e})}, \underline{\alpha}, \mathcal{H}^{(\mathrm{e})}\right)$ acting on $\mathcal{H}^{(\mathrm{e})}$. First, we define the $C^{*}$-representation $\pi_{(\mathrm{e})}: \mathfrak{A}^{(\mathrm{e})} \rightarrow \underline{\mathfrak{A}}^{(\mathrm{e})}$ given by

$$
\pi_{(\mathrm{e})}(A)=\underline{A}:=\left.A\right|_{\mathcal{H}^{(\mathrm{e})}}, \quad A \in \mathfrak{A}^{(\mathrm{e})}
$$


Due to the Reeh-Schlieder property of the vacuum and the fact that local operators are norm dense in $\mathfrak{A}$ we obtain that $\pi_{(\mathrm{e})}$ is a faithful representation. Thus there holds, by Proposition 2.3.3 of [BR],

$$
\|\underline{A}\|=\|A\|, \quad A \in \mathfrak{A}^{(\mathrm{e})} .
$$

Next, we note that the natural embedding $\mathcal{H}^{(\mathrm{e})} \hookrightarrow \mathcal{H}$ induces the embedding of the preduals $\iota_{(\mathrm{e})}: \mathcal{T}^{(\mathrm{e})} \hookrightarrow \mathcal{T}$, where $\mathcal{T}^{(\mathrm{e})}=B\left(\mathcal{H}^{(\mathrm{e})}\right)_{*}$. There clearly holds

$$
\iota_{(\mathrm{e})}(\underline{\varphi})(A)=\underline{\varphi}(\underline{A}), \quad A \in \mathfrak{A}^{(\mathrm{e})}, \underline{\varphi} \in \mathcal{T}^{(\mathrm{e})},
$$

and it is easy to see that $\iota_{(\mathrm{e})}$ is an isometry: The absolute value of any $\underline{\varphi} \in \mathcal{T}^{(\mathrm{e})}$ can be expressed as $\left.|\underline{\varphi}|=\sum_{i} p_{i} \mid \Psi_{i}\right)\left(\Psi_{i} \mid\right.$, where $p_{i}>0$ and $\Psi_{i} \in \mathcal{H}^{(\mathrm{e})}$ form an orthonormal system. Completing it to an orthonormal basis in $\mathcal{H}$, we obtain

$$
\left.\|\underline{\varphi}\|=\operatorname{Tr}|\underline{\varphi}|=\operatorname{Tr}\left|\iota_{(\mathrm{e})}(\underline{\varphi})\right|=\| \iota_{(\mathrm{e})} \underline{\varphi}\right) \| .
$$

Thus, making use of the fact that the embedding $\mathcal{H}^{(\mathrm{e})} \hookrightarrow \mathcal{H}$ preserves the energy of a vector, we obtain that

$$
\iota_{(\mathrm{e})}: \mathcal{T}_{E, 1}^{(\mathrm{e})} \rightarrow \mathcal{T}_{E, 1},
$$

what implies, together with relation ( $(\underline{B .2 .18})$, that $\|\underline{A}\|_{E, 2} \leq\|A\|_{E, 2}$ for square-integrable operators $A \in \hat{\mathfrak{A}}^{(\mathrm{e})}$.

Our next goal is to describe the field content of these theories. (Cf. Section 2.3 for a general discussion of this concept). For this purpose we introduce the domain $D_{S}=\{\Psi \in$ $\left.D_{F} \mid \Psi_{n} \in S\left(\mathbb{R}^{s \times n}\right)\right\}$ on which there acts the annihilation operator of the mode $\vec{p} \in \mathbb{R}^{s}$

$$
(a(\vec{p}) \Psi)_{n}\left(\vec{p}_{1}, \ldots, \vec{p}_{n}\right)=\sqrt{n+1} \Psi_{n+1}\left(\vec{p}, \vec{p}_{1}, \ldots, \vec{p}_{n}\right) .
$$

Clearly, on $D_{S}$ there holds the equality

$$
a(f)=\int d^{s} p \bar{f}(\vec{p}) a(\vec{p}), \quad f \in S\left(\mathbb{R}^{s}\right) .
$$

The adjoint $a^{*}(\vec{p})$ is only a quadratic form on $D_{S} \times D_{S}$. The Hamiltonian can be expressed as a quadratic form on this domain by

$$
H=\int d^{s} p \omega(\vec{p}) a^{*}(\vec{p}) a(\vec{p}) .
$$

Similarly, we define the pointlike localized canonical fields and momenta as quadratic forms on $D_{S} \times D_{S}$

$$
\begin{aligned}
& \phi_{+}(\vec{x})=\frac{1}{(2 \pi)^{s / 2}} \int \frac{d^{s} p}{\sqrt{2 \omega(\vec{p})}}\left(e^{-i \vec{p} \vec{x}} a^{*}(\vec{p})+e^{i \vec{p} \vec{x}} a(\vec{p})\right), \\
& \phi_{-}(\vec{x})=\frac{i}{(2 \pi)^{s / 2}} \int d^{s} p \sqrt{\frac{\omega(\vec{p})}{2}}\left(e^{-i \vec{p} \vec{x}} a^{*}(\vec{p})-e^{i \vec{p} \vec{x}} a(\vec{p})\right) .
\end{aligned}
$$

This terminology is justified by the fact that on this domain there holds for any $g \in S\left(\mathbb{R}^{s}\right)$

$$
\phi_{ \pm}(g)=\int d^{s} x \phi_{ \pm}(\vec{x}) g(\vec{x}) .
$$


Moreover, for any $s$-index $\kappa$, (see Section B.1 for our multiindex notation), we define the derivatives $\partial^{\kappa} \phi_{ \pm}:=\left.\partial^{\kappa} \phi_{ \pm}(\vec{x})\right|_{\vec{x}=0}$ and the corresponding Wick monomials

$$
: \partial^{\kappa_{1}^{+}} \phi_{+} \ldots \partial^{\kappa_{k+}^{+}} \phi_{+} \partial^{\kappa_{1}^{-}} \phi_{-} \ldots \partial^{\kappa_{k-}^{-}} \phi_{-}:
$$

which are also quadratic forms on $D_{S} \times D_{S}$. The Wick powers are defined by the standard prescription consisting in shifting all the creation operators to the left disregarding the commutator: 1 .

It is a well known fact (see e.g. Bos00]) that the Wick monomials can be extended by continuity to bounded functionals on $\mathcal{T}_{\infty}$ and the field content of scalar free field theory is given by

$$
\begin{array}{r}
\Phi_{\mathrm{FH}}=\operatorname{Span}\left\{: \partial^{\kappa_{1}^{+}} \phi_{+} \ldots \partial^{\kappa_{k^{+}}^{+}} \phi_{+} \partial^{\kappa_{1}^{-}} \phi_{-} \ldots \partial^{\kappa_{k^{-}}^{-}} \phi_{-}: \mid \kappa_{j^{ \pm}}^{ \pm} \in \mathbb{N}_{0}^{s}, j^{ \pm} \in\left\{1, \ldots, k^{ \pm}\right\},\right. \\
\left.k^{ \pm} \in \mathbb{N}_{0}\right\}(\mathrm{B}, 2
\end{array}
$$

i.e. it consists of finite linear combinations of the Wick monomials. Furthermore, it can be extracted from Section 7.4.2. of Bos00, that the field content of the sub-theory $\left(\mathfrak{A}^{(\mathrm{d})}, \alpha, \mathcal{H}\right)$ generated by the derivatives of the field has the form

$$
\begin{array}{r}
\Phi_{\mathrm{FH}}^{(\mathrm{d})}=\operatorname{Span}\left\{: \partial^{\kappa_{1}^{+}} \phi_{+} \ldots \partial^{\kappa_{k^{+}}^{+}} \phi_{+} \partial^{\kappa_{1}^{-}} \phi_{-} \ldots \partial^{\kappa_{k^{-}}^{-}} \phi_{-}: \mid \kappa_{j^{ \pm}}^{ \pm} \in \mathbb{N}_{0}^{s}, j^{ \pm} \in\left\{1, \ldots, k^{ \pm}\right\},\right. \\
\left.k^{ \pm} \in \mathbb{N}_{0},\left|\kappa_{j^{+}}^{+}\right|>0\right\} .(\mathrm{B} .
\end{array}
$$

(As mentioned above, in the massive case one can show that $\Phi_{\mathrm{FH}}^{(\mathrm{d})}=\Phi_{\mathrm{FH}}$, making use of the equation of motion. In the massless case, however, $\Phi_{\mathrm{FH}}^{(\mathrm{d})}$ is a proper subspace of $\left.\Phi_{\mathrm{FH}}\right)$. Finally, the even part $\left(\underline{\mathfrak{A}}^{(\mathrm{e})}, \underline{\alpha}, \mathcal{H}^{(\mathrm{e})}\right)$ of scalar free field theory has the following field content

$$
\Phi_{\mathrm{FH}}^{(\mathrm{e})}=\operatorname{Span}\left\{\frac{: \partial^{\kappa_{1}^{+}} \phi_{+} \ldots \partial^{\kappa_{k^{+}}^{+}} \phi_{+} \partial^{\kappa_{1}^{-}} \phi_{-} \ldots \partial^{\kappa_{k^{-}}^{-}} \phi_{-}: \mid}{k^{ \pm}} \in \kappa_{j^{ \pm}}^{ \pm} \in \mathbb{N}_{0}^{s}, j^{ \pm} \in\left\{1, \ldots, k^{ \pm}\right\}, k^{+}+k^{-} \text {is even }\right\},(\mathrm{B}, 2
$$

where the underlining indicates that the Wick monomials act on the Hilbert space $\mathcal{H}^{(\mathrm{e})}$.

To close the present section, we show that Condition $T$, stated in Section 2.3. holds in massive scalar free field theory and its even part.

Theorem B.2.1. Massive scalar free field theory and its even part satisfy Condition $T$ for any dimension of space $s \geq 1$.

Proof. First, we consider the full massive scalar free field theory. The $(0,0)$-component of the stress-energy tensor $T^{00} \in \Phi_{\mathrm{FH}}$ is given by

$$
T^{00}=\frac{1}{2}: \phi_{-}^{2}:+\frac{1}{2} \sum_{j=1}^{s}:\left(\partial_{j} \phi_{+}\right)^{2}:+\frac{1}{2} m^{2}: \phi_{+}^{2}: .
$$

The standard computation, which makes use of representation (B.2.23) of the Hamiltonian, gives for $\Psi, \Phi \in D_{S}$

$$
\int d^{s} x\left(\Psi \mid T^{00}(\vec{x}) \Phi\right)=(\Psi \mid H \Phi)
$$

\footnotetext{
${ }^{1}$ We warn the reader that the Wick ordering is not linear on the algebra of (smeared) creation and annihilation operators.
} 
Certainly, equality (B.2.32) still holds if $T^{00}$ is replaced with $T^{00}(g)$, where the timesmearing function $g$ is specified in Condition T. It is shown in Appendix D that massive scalar free field theory satisfies Condition $L^{(1)}$ which ensures that $\left\|T^{00}(g)\right\|_{E, 1}<\infty$. (See relation (2.3.15D). Due to this bound and the fact that $D_{S} \cap P_{E} \mathcal{H}$ is dense in $P_{E} \mathcal{H}$, we obtain for any $\Psi, \Phi \in P_{E} \mathcal{H}$

$$
\int d^{s} x\left(\Psi \mid T^{00}(g)(\vec{x}) \Phi\right)=(\Psi \mid H \Phi) .
$$

Making use of the fact that any functional $\varphi \in \mathcal{T}_{E}$ is of the form $\varphi(\cdot)=\sum_{j}\left(\Psi_{j} \mid \cdot \Phi_{j}\right)$, where $\Psi_{j}, \Phi_{j} \in P_{E} \mathcal{H}$ and $\sum_{j}\left\|\Psi_{j}\right\|\left\|\Phi_{j}\right\|<\infty[\mathrm{BR}$, we conclude the proof for the full theory. The even part is treated analogously, exploiting the fact that $\underline{T^{00}}$ is an element of $\Phi_{\mathrm{FH}}^{(\mathrm{e})}$ and restricting attention to $\Psi, \Phi \in \mathcal{H}^{(\mathrm{e})}$.

\section{B.3 Special Functionals on Local Algebra}

In this section we construct suitable functionals on the local algebra $\mathfrak{A}(\mathcal{O}(r))$, estimate their norms and establish certain energy bounds. For this last purpose we introduce the Sobolev spaces [RS2] given, for any $l \geq 0$, by

$$
L^{2}\left(\mathbb{R}^{s}, d^{s} x\right)_{l}=\left\{\left.f \in L^{2}\left(\mathbb{R}^{s}, d^{s} x\right)\left|\int d^{s} p\left(1+|\vec{p}|^{2}\right)^{l}\right| \tilde{f}(\vec{p})\right|^{2}<\infty\right\} .
$$

These spaces are equipped with the norm

$$
\|f\|_{2, l}=\left(\int d^{s} p\left(1+|\vec{p}|^{2}\right)^{l}|\tilde{f}(\vec{p})|^{2}\right)^{\frac{1}{2}} .
$$

Clearly, $\|\cdot\|_{2,0}=\|\cdot\|_{2}$. The goal of this section is to prove the following proposition which is a slight generalization of Lemmas 7.8 and 7.9 of [Bos00].

Proposition B.3.1. Let $l \geq 0$ and $\left\{b_{i}^{+}\right\}_{1}^{\infty},\left\{b_{i}^{-}\right\}_{1}^{\infty}$ be two sequences of J-invariant vectors from $L^{2}\left(\mathbb{R}^{s}, d^{s} x\right)_{l}$. Let $\mu^{+}, \mu^{-}$be two multiindices, $f^{ \pm} \in \stackrel{\mathcal{L}}{r}_{r}^{ \pm}$and $f=f^{+}+i f^{-}$. Then there exists a normal functional $\sigma_{\mu^{+}, \mu^{-}}$on $B(\mathcal{H})$ s.t.

$$
\begin{aligned}
\sigma_{\mu^{+}, \mu^{-}}(W(f)) & =e^{-\frac{1}{2}\|f\|_{2}^{2}\left\langle b^{+} \mid f^{+}\right\rangle^{\mu^{+}}\left\langle b^{-} \mid f^{-}\right\rangle^{\mu^{-}}} \\
\left\|R^{-l} \sigma_{\mu^{+}, \mu^{-}} R^{-l}\right\| & \leq\left(2 c_{l}\right)^{\left|\mu^{+}\right|+\left|\mu^{-}\right|} \sqrt{\left(\left|\mu^{+}\right|+\left|\mu^{-}\right|\right) !}\left\|b^{+}\right\|_{2, l}^{\mu^{+}}\left\|b^{-}\right\|_{2, l}^{\mu^{-}},
\end{aligned}
$$

where $R=(1+H)^{-1}, c_{l}=\left(12+2 m^{2}\right)^{l / 2}$, and $m$ is the mass of the theory. Moreover, if $b_{i}^{+}=b_{i}^{-}$for any $i \in \mathbb{N}$ and $\left\{b_{i}^{+}\right\}_{1}^{\infty}$ form an orthonormal system of vectors in $L^{2}\left(\mathbb{R}^{s}, d^{s} x\right)$, then there holds

$$
\left\|\sigma_{\mu^{+}, \mu^{-}}\right\| \leq 4^{\left|\mu^{+}\right|+\left|\mu^{-}\right|} \sqrt{\mu^{+} ! \mu^{-} !}
$$

For the proof of this proposition we need two simple lemmas. First, we introduce energy damping operators which are more convenient than the resolvents $R$ considered above.

Lemma B.3.2. Let $l \geq 0$ and $G_{l}=\int d^{s} p \log (2+\omega(\vec{p}))^{l} a^{*}(\vec{p}) a(\vec{p})$. Then there holds

$$
\left\|R^{-l} e^{-G_{l}}\right\| \leq 1 \text {. }
$$


Proof. Let $n \geq 1$ and $\Psi_{n}$ be an $n$-particle (Schwartz-class) wavefunction. Then there holds

$$
\begin{aligned}
\left(\Psi_{n} \mid R^{-l} e^{-G_{l}} \Psi_{n}\right) & =\int d^{s} p_{1} \ldots d^{s} p_{n}\left|\Psi_{n}\left(\vec{p}_{1}, \ldots, \vec{p}_{n}\right)\right|^{2} \frac{\left(1+\omega\left(\vec{p}_{1}\right)+\cdots+\omega\left(\vec{p}_{n}\right)\right)^{l}}{\left(2+\omega\left(\vec{p}_{1}\right)\right)^{l} \ldots\left(2+\omega\left(\vec{p}_{n}\right)\right)^{l}} \\
& \leq\left\|\Psi_{n}\right\|^{2},
\end{aligned}
$$

where we made use of the fact that $R^{-l} e^{-G_{l}}$ is a positive operator and that $(x+y) \leq x y$ for any $x \geq 2, y \geq 2$. We can thus drop the restriction that $\Psi_{n}$ is a Schwartz-class function. Now making use of the fact that any vector $\Psi$ in the Fock space can be expressed as $\Psi=\sum_{n=0}^{\infty} \Psi_{n}$, where $\Psi_{0}=c \Omega$ for some $c \in \mathbb{C}$ and $\|\Psi\|^{2}=\sum_{n=0}^{\infty}\left\|\Psi_{n}\right\|<\infty$, we obtain from (B.3.7)

$$
\left(\Psi \mid R^{-l} e^{-G_{l}} \Psi\right) \leq\|\Psi\|^{2}
$$

what concludes the proof.

Next, we recall the elementary combinatorial Lemma 7.6 of Bos00] which gives useful bounds on the lengths of certain vectors in the Fock space.

Lemma B.3.3. For any family of vectors $b_{1} \ldots b_{n} \in L^{2}\left(\mathbb{R}^{s}, d^{s} x\right)$ there holds

$$
\left\|a\left(b_{1}\right) \ldots a\left(b_{k}\right) a^{*}\left(b_{k+1}\right) \ldots a^{*}\left(b_{n}\right) \Omega\right\| \leq \sqrt{n !}\left\|b_{1}\right\| \ldots\left\|b_{n}\right\| .
$$

Moreover, if $\left\{b_{i}\right\}_{1}^{\infty}$ form an orthonormal system of vectors in $L^{2}\left(\mathbb{R}^{s}, d^{s} x\right)$ and $\alpha, \beta$ are multiindices, then there holds

$$
\left\|a(e)^{\alpha} a^{*}(e)^{\beta} \Omega\right\| \leq \sqrt{(\alpha+\beta) !} .
$$

After this preparation we are in position to prove the main result of this section.

Proof of Proposition B.3.1 This is a slight generalization of the argument from Appendix 4.2.B of [Bos00]. Consider the generating functional

$$
F(\underline{u}, \underline{w})(A)=\left(\Omega \mid e^{\frac{1}{2} a\left(i \underline{u} \underline{b}^{+}-\underline{w} \underline{b}^{-}\right)} e^{\frac{1}{2} a^{*}\left(i \underline{u} \underline{b}^{+}-\underline{w} \underline{b}^{-}\right)} A e^{-\frac{1}{2} a^{*}\left(i \underline{u} \underline{b}^{+}-\underline{w} \underline{b}^{-}\right)} \Omega\right),
$$

where $A$ is a bounded operator on the Fock space and $\underline{u}=\left\{u_{i}\right\}_{1}^{\infty}, \underline{w}=\left\{w_{i}\right\}_{1}^{\infty}$ are sequences of real numbers with only a finite number of entries different from zero. We denote $\underline{u b}^{+}=\sum_{i} u_{i} b_{i}^{+}, \underline{w b}^{-}=\sum_{i} w_{i} b_{i}^{-}$and define the exponentials above by their Taylor series expansions which converge in view of Lemma B.3.3. In particular, for $A=W(f)$ we obtain

$$
F(\underline{u}, \underline{w})(W(f))=e^{-\frac{1}{2}\|f\|_{2}^{2}} e^{\underline{u}\left\langle\underline{b}^{+} \mid f^{+}\right\rangle+\underline{w}\left\langle\underline{b}^{-} \mid f^{-}\right\rangle} .
$$

Differentiating the above formula, we obtain

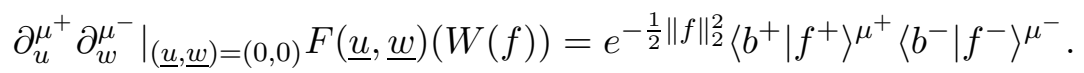

Therefore, we can define

$$
\sigma_{\mu^{+}, \mu^{-}}(A):=\left.\partial_{u}^{\mu^{+}} \partial_{w}^{\mu^{-}}\right|_{(\underline{u}, \underline{w})=(0,0)} F(\underline{u}, \underline{w})(A) .
$$


From this definition and formula (B.3.11) we obtain

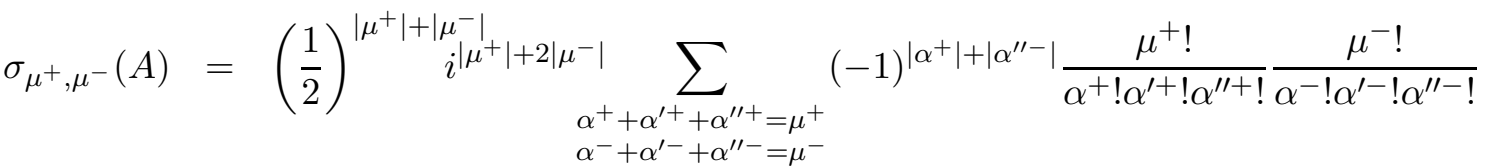

$$
\begin{aligned}
& \text { - }\left(\Omega \mid a\left(b^{+}\right)^{\alpha^{+}} a\left(b^{-}\right)^{\alpha^{-}} a^{*}\left(b^{+}\right)^{\alpha^{++}} a^{*}\left(b^{-}\right)^{\alpha^{-}} A a^{*}\left(b^{+}\right)^{\alpha^{\prime \prime+}} a^{*}\left(b^{-}\right)^{\alpha^{\prime \prime}-} \Omega\right) \\
& =\left(\frac{1}{2}\right)^{\left|\mu^{+}\right|+\left|\mu^{-}\right|} i^{\left|\mu^{+}\right|+2\left|\mu^{-}\right|} \sum_{\bar{\alpha}+\bar{\alpha}^{\prime}+\bar{\alpha}^{\prime \prime}=\bar{\mu}}(-1)^{\left|\alpha^{+}\right|+\left|\alpha^{\prime \prime}-\right|} \frac{\bar{\mu} !}{\bar{\alpha} ! \bar{\alpha}^{\prime} ! \bar{\alpha}^{\prime \prime} !} . \\
& \cdot\left(\Omega \mid a(b)^{\bar{\alpha}} a^{*}(b)^{\bar{\alpha}^{\prime}} A a^{*}(b)^{\bar{\alpha}^{\prime \prime}} \Omega\right) .
\end{aligned}
$$

Here $\bar{\alpha}=\left(\alpha^{+}, \alpha^{-}\right)$etc. are 2-multiindices. Since the operator $G_{l}$, introduced in Lemma B.3.2 satisfies $\left\|R^{-l} e^{-G_{l}}\right\| \leq 1$ and $e^{G_{l}} a^{*}(b) e^{-G_{l}}=a^{*}\left((2+\omega)^{l} b\right)$, there holds

$$
\begin{aligned}
& \sup _{A \in B(\mathcal{H})_{1}}\left|\left(\Omega \mid a(b)^{\bar{\alpha}} a^{*}(b)^{\bar{\alpha}^{\prime}} R^{-l} A R^{-l} a^{*}(b)^{\bar{\alpha}^{\prime \prime}} \Omega\right)\right| \\
\leq & \sup _{A \in B(\mathcal{H})_{1}}\left|\left(\Omega \mid a\left((2+\omega)^{-l} b\right)^{\bar{\alpha}} a^{*}\left((2+\omega)^{l} b\right)^{\bar{\alpha}^{\prime}} A a^{*}\left((2+\omega)^{l} b\right)^{\bar{\alpha}^{\prime \prime}} \Omega\right)\right| \\
\leq & c_{l}^{\left|\mu^{+}\right|+\left|\mu^{-}\right|} \sqrt{\left(\left|\mu^{+}\right|+\left|\mu^{-}\right|\right) !}\left\|b^{+}\right\|_{2, l}^{\mu^{+}}\left\|b^{-}\right\|_{2, l}^{\mu^{-}},
\end{aligned}
$$

where in the last step we made use of estimate (B.3.9) and $c_{l}$ is defined in the statement of the proposition. It follows from this relation and formula $(\mathrm{B} .3 .15)$ that $R^{-l} \sigma_{\mu^{+}, \mu^{-}} R^{-l}$ is a bounded functional. Making use of relation (B.3.15) and exploiting the following formula, valid for any $n \in \mathbb{N}$,

$$
\sum_{\substack{a, a^{\prime}, a^{\prime \prime} \in \mathbb{N}_{0} \\ a+a^{\prime}+a^{\prime \prime}=n}} \frac{n !}{a ! a^{\prime} ! a^{\prime \prime !} !}=3^{n},
$$

we prove estimate (B.3.4) from the statement of the proposition. In order to verify relation (B.3.5), suppose that $b_{i}^{+}=b_{i}^{-}$for $i \in \mathbb{N}$ and $\left\{b_{i}^{+}\right\}_{1}^{\infty}$ form an orthogonal system. Then estimate (B.3.10) gives

$$
\sup _{A \in B(\mathcal{H})_{1}}\left|\left(\Omega \mid a(b)^{\bar{\alpha}} a^{*}(b)^{\bar{\alpha}^{\prime}} A a^{*}(b)^{\bar{\alpha}^{\prime \prime}} \Omega\right)\right| \leq \sqrt{\left(\mu^{+}+\mu^{-}\right) !} \leq 2^{\left|\mu^{+}\right|+\left|\mu^{-}\right|} \sqrt{\mu^{+} ! \mu^{-} !}
$$

Substituting this bound to formula (B.3.15) and making use again of identity (B.3.17), we conclude the proof of the proposition.

\section{B.4 Expansions in Single-Particle Space}

In this section we derive two expansions of the functions $F^{ \pm} \in D\left(\mathcal{O}_{r}\right)_{\mathbb{R}}$ which enter into definition B.2.6) of the local algebra. The first expansion relies on the fact that $\tilde{F}^{ \pm}$are analytic functions and thus can be expanded into convergent Taylor series. The coefficients of these expansions are given in terms of vectors $\left\{\tilde{b}_{\kappa_{j}, r}^{ \pm}\right\}_{1}^{\infty}$ defined by (B.4.1) below. We show in Lemma B.4.3 stated below, that these vectors have finite Sobolev norms. Thus the corresponding functionals $\sigma_{\mu^{+}, \mu^{-}}$, constructed in Proposition B.3.1 satisfy the energy 
bounds (B.3.4). Due to the rapid growth with $\left|\mu^{+}\right|+\left|\mu^{-}\right|$, this estimate does not suffice to establish the convergence of the sum (B.0.2) in the massless case. In order to exploit the more tame estimate (B.3.5), in the second part of this section we construct a suitable orthonormal basis expansion.

\section{B.4.1 Taylor Expansion}

We introduce two approximate characteristic functions: $\vec{p} \rightarrow \chi_{E}(\vec{p}) \in C_{0}^{\infty}\left(\mathbb{R}^{s}\right)$ which is equal to one on the set $\left\{\vec{p} \in \mathbb{R}^{s}|| \omega(\vec{p}) \mid \leq E\right\}$ and $\vec{x} \rightarrow \chi\left(\mathcal{O}_{r}\right)(\vec{x}) \in C_{0}^{\infty}\left(\mathbb{R}^{s}\right)$ which is equal to one on the ball $\mathcal{O}_{r}$, and define, for any $s$-index $\kappa$, the functions from $L^{2}\left(\mathbb{R}^{s}, d^{s} p\right)$

$$
\begin{aligned}
\tilde{b}_{\kappa, r}^{ \pm}(\vec{p}) & =\frac{1}{(2 \pi)^{\frac{s}{2}}} \frac{\left.\omega(\vec{p})^{ \pm \frac{1}{2}} \widehat{x^{\kappa} \chi\left(\mathcal{O}_{r}\right.}\right)(\vec{p})}{\kappa !}, \\
\tilde{h}_{\kappa, E}^{ \pm}(\vec{p}) & =(-1)^{|\kappa|} \omega(\vec{p})^{\mp \frac{1}{2}}(i p)^{\kappa} \chi_{E}(\vec{p}) .
\end{aligned}
$$

We recall that $f^{ \pm} \in \mathcal{L}_{r}^{ \pm}$have the form $\tilde{f}^{ \pm}=\omega^{\mp \frac{1}{2}} \tilde{F}^{ \pm}$, where $F^{ \pm} \in D\left(\mathcal{O}_{r}\right)_{\mathbb{R}}$, set $\tilde{F}_{E}^{ \pm}:=$ $\chi_{E} \tilde{F}^{ \pm}$and note that the functions $\tilde{F}^{ \pm}$are analytic. From their Taylor expansions we obtain Bos00]

$$
\omega^{\mp \frac{1}{2}} \tilde{F}_{E}^{ \pm}=\chi_{E} \tilde{f}^{ \pm}=\sum_{\kappa}\left\langle b_{\kappa, r}^{ \pm} \mid f^{ \pm}\right\rangle \tilde{h}_{\kappa, E}^{ \pm}=\sum_{j=1}^{\infty}\left\langle b_{\kappa_{j}, r}^{ \pm} \mid f^{ \pm}\right\rangle \tilde{h}_{\kappa_{j}, E}^{ \pm},
$$

where in the second step we numbered the $s$-indices $\kappa$ with some index $j \in \mathbb{N}$ in such a way that $\kappa_{1}=0$. There holds the following proposition.

Proposition B.4.1. [Bos00] Expansion B.4.3) converges in $L^{2}\left(\mathbb{R}^{s}, d^{s} p\right)$.

This statement follows from estimate (B.4.17) below which relies on the bounds on the norms of functions (B.4.1) and (B.4.2), established in Lemmas B.4.3 and B.4.4 respectively. Actually, we derive here more general estimates on the Sobolev norms of these functions, (see definition (B.3.2) $)$, which appear in Proposition B.3.1 above. First, we note the following auxiliary fact.

Lemma B.4.2. Let $\chi \in C_{0}^{\infty}\left(\mathbb{R}^{s}\right)$. Then, for any $s$-index $\kappa, i \in\{1, \ldots, s\}$ and $n \in \mathbb{N}$, there holds

$$
\int d^{s} x\left|\partial_{x_{i}}^{n}\left(x^{\kappa} \chi(\vec{x})\right)\right|^{2} \leq\left(c_{n}\right)^{|\kappa|+1}
$$

for some constant $c_{n}$, independent of $\kappa$.

Proof. We note the following identity which follows from the Leibniz rule

$$
\partial_{x_{i}}^{n}\left(x^{\kappa} \chi\right)(\vec{x})=x^{\hat{\kappa}} \sum_{k=0}^{\min (n, \kappa(i))} \frac{n !}{(n-k) ! k !} \frac{\kappa(i) !}{(\kappa(i)-k) !} x_{i}^{\kappa(i)-k} \partial_{x_{i}}^{n-k} \chi(\vec{x}),
$$

where the $s$-index $\hat{\kappa}$ is obtained from $\kappa$ by setting $\kappa(i)=0$. There easily follows the bound

$$
\int d^{s} x\left|\partial_{x_{i}}^{n}\left(x^{\kappa} \chi(\vec{x})\right)\right|^{2} \leq 2^{n}\left(c_{n}\right)^{|\kappa|} \int d^{s} x\left(\sum_{k=0}^{n}\left|\partial_{x_{i}}^{k} \chi(\vec{x})\right|\right)^{2}
$$

for some constant $c_{n}$, independent of $\kappa$.

Now we are ready to prove the required bounds on the Sobolev norms of $b_{\kappa}^{ \pm}$. 
Lemma B.4.3. For any $l \geq 0$ the functions $b_{\kappa, r}^{ \pm}$, defined by B.4.1), satisfy the bound

$$
\left\|b_{\kappa, r}^{ \pm}\right\|_{2, l} \leq \frac{\left(c_{l, r}\right)^{|\kappa|+1}}{\kappa !}
$$

where the constant $c_{l, r}$, is independent of $\kappa$.

Proof. First, we note that $\left\|b_{\kappa, r}^{+}\right\|_{2, l} \leq(1+m)^{1 / 4}\left\|b_{\kappa, r}^{-}\right\|_{2, l+\frac{1}{2}}$, so it suffices to consider the (-) case. Clearly $l \rightarrow\left\|b_{\kappa, r}^{-}\right\|_{2, l}$ is a monotonically increasing function, so it is enough to establish the bound for $l=n+\frac{1}{2}, n \in \mathbb{N}$. We consider the expression

$$
\begin{aligned}
& \left.\left\|\omega^{-\frac{1}{2}} \widetilde{x^{\kappa} \chi\left(\mathcal{O}_{r}\right)}\right\|_{2, n+\frac{1}{2}}^{2}=\left(\widetilde{x^{\kappa} \chi\left(\mathcal{O}_{r}\right.}\right) \mid \omega(\vec{p})^{-1}\left(1+|\vec{p}|^{2}\right)^{n+\frac{1}{2}} \widetilde{x^{\kappa} \chi\left(\mathcal{O}_{r}\right)}\right)
\end{aligned}
$$

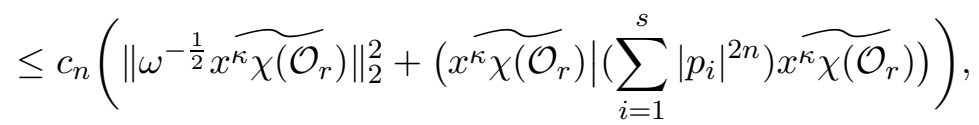

where on the r.h.s. above we mean the scalar product in $L^{2}\left(\mathbb{R}^{s}, d^{s} p\right)$ and the constant $c_{n}$ depends only on $n$ and $s$. In order to study the first term on the r.h.s. above, we write $\omega^{-\frac{1}{2}}=\left(\omega^{-\frac{1}{2}}\right)_{+}+\left(\omega^{-\frac{1}{2}}\right)_{-}$, where $\left(\omega^{-\frac{1}{2}}\right)_{ \pm}(\vec{p})=\omega(\vec{p})^{-\frac{1}{2}} \theta( \pm(|\vec{p}|-1))$. There clearly holds

$$
\begin{aligned}
& \left.\|\left(\omega^{-\frac{1}{2}}\right)_{+} \widetilde{x^{\kappa} \chi\left(\mathcal{O}_{r}\right.}\right)\left\|_{2} \leq\right\| x^{\kappa} \chi\left(\mathcal{O}_{r}\right) \|_{2} \leq \frac{1}{2}\left(c_{r}\right)^{|\kappa|+1}, \\
& \left.\left.\|\left(\omega^{-\frac{1}{2}}\right)_{-} \widetilde{x^{\kappa} \chi\left(\mathcal{O}_{r}\right.}\right)\left\|_{2} \leq\right\| \widetilde{x^{\kappa} \chi\left(\mathcal{O}_{r}\right.}\right)\left\|_{\infty}\right\|\left(\omega^{-\frac{1}{2}}\right)_{-} \|_{2} \leq \frac{1}{2}\left(c_{r}\right)^{|\kappa|+1},
\end{aligned}
$$

for some constant $c_{r} \geq 0$, independent of $\kappa$, and therefore

$$
\left\|\omega^{-\frac{1}{2}} \widetilde{x^{\kappa} \chi\left(\mathcal{O}_{r}\right)}\right\|_{2} \leq\left(c_{r}\right)^{|\kappa|+1} .
$$

Next, we study the second term on the r.h.s. of $(\mathbb{B} .4 .8)$. Making use of Lemma B.4.2 we obtain

$$
\left.\left.\widetilde{\left(x^{\kappa} \chi\left(\mathcal{O}_{r}\right)\right.}|| p_{i}\right|^{2 n} \widetilde{x^{\kappa} \chi\left(\mathcal{O}_{r}\right)}\right)=\int d^{s} x\left|\partial_{x_{i}}^{n}\left(x^{\kappa} \chi\left(\mathcal{O}_{r}\right)\right)(\vec{x})\right|^{2} \leq\left(c_{n, r}\right)^{|\kappa|+1},
$$

where the constant $c_{n, r}$ does not depend on $\kappa$. This concludes the proof of the lemma.

While the above result holds for any $m \geq 0$, in the next statement, concerning the Sobolev norms of the functions $\tilde{h}_{\kappa, E}^{ \pm}$, we have to make a distinction between the massive and the massless case.

Lemma B.4.4. In massive scalar free field theory for $s \geq 1$ the functions $\tilde{h}_{\kappa, E}^{ \pm}$, defined by relation B.4.2), satisfy, for any $\lambda \geq 0, \beta \in \mathbb{R}$,

$$
\left\|\omega^{-\beta} \tilde{h}_{\kappa, E}^{ \pm}\right\|_{2, \lambda} \leq\left(c_{\lambda, \beta, E}\right)^{|\kappa|+1},
$$

where the constants $c_{\lambda, \beta, E}$ are independent of $\kappa$. In massless scalar free field theory for $s \geq 3$ the bound B.4.13) holds (in particular) in the following two cases:

(a) For any $\beta<1$ and $\lambda=0$.

(b) For $\beta=\mp \frac{1}{2}$ and any $\lambda \geq 0$. (The \pm signs are correlated with these appearing in formula B.4.13). 
Proof. We define $\chi_{E}^{ \pm}(\vec{p}):=\omega(\vec{p})^{-\beta \mp \frac{1}{2}} \chi_{E}(\vec{p})$. Then

$$
\left(\omega^{-\beta} \tilde{h}_{\kappa, E}^{ \pm}\right)(\vec{p})=(-1)^{|\kappa|}(i p)^{\kappa} \chi_{E}^{ \pm}(\vec{p}) .
$$

We first consider the case $m=0$. Then the functions $\chi_{E}^{ \pm}$are square-integrable for $\beta<1$ and there holds for some constant $c_{E}$, independent of $\kappa$,

$$
\left\|\omega^{-\beta} \tilde{h}_{\kappa, E}^{ \pm}\right\|_{2} \leq c_{E}^{|\kappa|}\left\|\chi_{E}^{ \pm}\right\|_{2}
$$

what proves part (a) and part (b) for $\lambda=0$. For $m>0$ inequality (B.4.15) holds for any $\beta \in \mathbb{R}$.

In the massive case and the case considered in part (b), $\vec{p} \rightarrow \chi_{E}^{ \pm}(\vec{p})$ are smooth, compactly supported functions. It suffices to take into account $\lambda \in \mathbb{N}$, since the functions $\lambda \rightarrow\left\|\omega^{-\beta} \tilde{h}_{\kappa, E}^{ \pm}\right\|_{2, \lambda}$ are monotonically increasing. By Hölder's inequality applied to the term $\left(1+|\vec{x}|^{2}\right)^{\lambda}$, identity (B.4.14), Lemma B.4.2 and relation (B.4.15) there holds the following bound

$$
\left\|\omega^{-\beta} \tilde{h}_{\kappa, E}^{ \pm}\right\|_{2, \lambda}^{2} \leq c_{\lambda}\left(\left\|\omega^{-\beta} \tilde{h}_{\kappa, E}^{ \pm}\right\|_{2}^{2}+\sum_{i=1}^{s} \int d^{s} p\left|\partial_{p_{i}}^{\lambda}\left(p^{\kappa} \chi_{E}^{ \pm}(\vec{p})\right)\right|^{2}\right) \leq\left(c_{\lambda, \beta, E}\right)^{|\kappa|+1},(B
$$

where the constant $c_{\lambda, \beta, E}$ is independent of $\kappa$.

From Lemmas B.4.3 and B.4.4 we obtain the bound for any combination of \pm -signs, any $0 \leq \beta \leq 1$ and $0<p \leq 1$

$$
\sum_{\kappa \in \mathbb{N}_{0}^{s}}\left\|b_{\kappa, r}^{ \pm}\right\|_{2}^{p}\left\|\omega^{-\beta} \tilde{h}_{\kappa, E}^{ \pm}\right\|_{2}^{p} \leq \sum_{\kappa \in \mathbb{N}_{0}^{s}} \frac{\left(c_{0, r} c_{0, \beta, E}\right)^{p(|\kappa|+1)}}{(\kappa !)^{p}}<\infty .
$$

Thus we have proven Proposition B.4.1.

\section{B.4.2 Orthonormal Basis Expansion}

Let $Q_{E}$ be the projection on the single-particle space onto states of energy lower than $E$. Let $h_{r} \in D\left(\mathcal{O}_{r}\right)_{\mathbb{R}}$ be s.t. $\tilde{h}_{r}>0$. We introduce the closed, linear subspaces $\mathcal{L}_{r}^{ \pm}=$ $\left[\omega^{ \pm \frac{1}{2}} \widetilde{D}\left(\mathcal{O}_{r}\right)\right]$ in $L^{2}\left(\mathbb{R}^{s}, d^{s} p\right)$ and denote the respective projections by the same symbols. We choose $\frac{1}{2} \leq \gamma<\frac{s-1}{2}$ and define operators $T_{E, \pm}=\omega^{-\frac{1}{2}} Q_{E} \mathcal{L}_{r}^{ \pm}, T_{h, \pm}=\omega^{-\gamma} \tilde{h}_{r}^{1 / 2} \mathcal{L}_{r}^{ \pm}$, where $\tilde{h}_{r}$ is the corresponding multiplication operator in momentum space. We recall that the $p$-norm of an operator $A$ is given, for any $p>0$, by $\|A\|_{p}=\left\||A|^{p}\right\|_{1}^{1 / p}$, where $\|\cdot\|_{1}$ denotes the trace norm. By a slight modification of Lemma 3.5 from BP90, one obtains the following result:

Lemma B.4.5. For any $p>0$ the operators $T_{E, \pm}$ and $T_{h, \pm}$ are $p$-nuclear i.e. there holds

$$
\begin{aligned}
\left\|T_{E, \pm}\right\|_{p} & <\infty \\
\left\|T_{h, \pm}\right\|_{p} & <\infty
\end{aligned}
$$


Proof. In order to show that the operators $T_{h, \pm}$ are $p$-nuclear for any $p>0$, it suffices to demonstrate that their adjoints $T_{h, \pm}^{*}$ are products of an arbitrary number of HilbertSchmidt operators. (The Hilbert-Schmidt property is preserved under the adjoint operation due to the cyclicity of the trace). We set, as in BP90, for $i \in \mathbb{N}$

$$
\begin{aligned}
h_{i} & =\omega\left(1+\omega^{2}\right)^{(i-1) s} \chi\left(\mathcal{O}_{r}\right)\left(1+\omega^{2}\right)^{-i s} \omega^{-1} \\
k_{i} & =\left(1+\omega^{2}\right)^{(i-1) s} \chi\left(\mathcal{O}_{r}\right)\left(1+\omega^{2}\right)^{-i s} .
\end{aligned}
$$

These operators, and also $\omega^{-\frac{1}{2}} h_{1}, \omega^{-\frac{1}{2}} k_{1}$, are in the Hilbert-Schmidt class BP90. For any $n \in \mathbb{N}$ there hold the identities

$$
\begin{aligned}
& T_{h,+}^{*}=\mathcal{L}_{r}^{+} \omega^{-\frac{1}{2}} h_{1} \ldots h_{n} \omega^{-\gamma+\frac{1}{2}}\left(1+\omega^{2}\right)^{n s} \tilde{h}^{1 / 2}, \\
& T_{h,-}^{*}=\mathcal{L}_{r}^{-} \omega^{-\frac{1}{2}} k_{1} \ldots k_{n} \omega^{-\gamma+\frac{1}{2}}\left(1+\omega^{2}\right)^{n s} \tilde{h}^{1 / 2},
\end{aligned}
$$

so it suffices to check that $h_{n} \omega^{-\gamma+\frac{1}{2}}$ and $k_{n} \omega^{-\gamma+\frac{1}{2}}$ are Hilbert-Schmidt. Since the HilbertSchmidt norm $\|\cdot\|_{\text {HS }}$ of an operator is equal to the $L^{2}$-norm of its integral kernel, we obtain

$$
\begin{array}{r}
\left\|h_{n} \omega^{-\gamma+\frac{1}{2}}\right\|_{\mathrm{HS}}^{2}=(2 \pi)^{-s} \int d^{s} p d^{s} q \omega(\vec{p})^{2}\left(1+\omega(\vec{p})^{2}\right)^{2(n-1) s} \widetilde{\left.\chi_{\left(\mathcal{O}_{r}\right)}(\vec{p}-\vec{q})\right|^{2}} \\
\cdot \frac{1}{\left(1+\omega(\vec{q})^{2}\right)^{2 n s}} \frac{1}{\omega(\vec{q})^{2 \gamma+1}} .
\end{array}
$$

Making use of the following two identities (see e.g. formula (7.2.109) of [Bos00] for the proof of the first statement)

$$
\begin{gathered}
\frac{1+\omega(\vec{p})^{2}}{1+\omega(\vec{q})^{2}} \leq(|\vec{p}-\vec{q}|+1)^{2}, \\
\omega(\vec{p})^{2} \leq 2\left(\omega(\vec{p}-\vec{q})^{2}+\omega(\vec{q})^{2}\right),
\end{gathered}
$$

we arrive at the bound

$$
\begin{aligned}
& \left\|h_{n} \omega^{-\gamma+\frac{1}{2}}\right\|_{\mathrm{HS}}^{2} \\
\leq & 2(2 \pi)^{-s} \int d^{s} p d^{s} q \frac{1}{\left(1+\omega(\vec{q})^{2}\right)^{2 s}} \frac{1}{\omega(\vec{q})^{2 \gamma-1}}(|\vec{p}|+1)^{4(n-1) s} \widetilde{\left.\chi_{\left(\mathcal{O}_{r}\right)}(\vec{p})\right|^{2}} \\
+ & 2(2 \pi)^{-s} \int d^{s} p d^{s} q \frac{1}{\left(1+\omega(\vec{q})^{2}\right)^{2 s}} \frac{1}{\omega(\vec{q})^{2 \gamma+1}} \omega(\vec{p})^{2}(|\vec{p}|+1)^{4(n-1) s}\left|\widetilde{\chi\left(\mathcal{O}_{r}\right)}(\vec{p})\right|^{2} .
\end{aligned}
$$

These integrals are clearly convergent for $0 \leq \gamma<\frac{s-1}{2}$. The (simpler) case of $k_{n} \omega^{-\gamma+\frac{1}{2}}$ is treated analogously. Finally, the $p$-nuclearity of the operators $T_{E, \pm}$ follows from the fact that $Q_{E} \tilde{h}_{r}^{-1}$ is a bounded operator and there holds $T_{E, \pm}=\left(Q_{E} \tilde{h}_{r}^{-1}\right) T_{h, \pm}$ for $\gamma=\frac{1}{2}$.

We define the operator $T$ as follows

$$
T=\left(\left|T_{E,+}\right|^{2}+\left|T_{E,-}\right|^{2}+\left|T_{h,+}\right|^{2}+\left|T_{h,-}\right|^{2}\right)^{\frac{1}{2}} .
$$

Making use of the fact Ko84 that for any $0<p \leq 1$ and any pair of positive operators $A, B$ s.t. $A^{p}, B^{p}$ are trace-class, there holds $\left\|(A+B)^{p}\right\|_{1} \leq\left\|A^{p}\right\|_{1}+\left\|B^{p}\right\|_{1}$, we get

$$
\|T\|_{p}^{p} \leq\left\|T_{E,+}\right\|_{p}^{p}+\left\|T_{E,-}\right\|_{p}^{p}+\left\|T_{h,+}\right\|_{p}^{p}+\left\|T_{h,-}\right\|_{p}^{p}
$$


Since $T$ commutes with the operator $J$ of complex conjugation in configuration space, it has a $J$-invariant orthonormal basis of eigenvectors $\left\{e_{j}\right\}_{1}^{\infty}$ and we denote the corresponding eigenvalues by $\left\{t_{j}\right\}_{1}^{\infty}$. As we will see in the next section, the expansion

$$
Q_{E} f^{ \pm}=\sum_{j=1}^{\infty}\left\langle e_{j} \mid f^{ \pm}\right\rangle Q_{E} \mathcal{L}_{r}^{ \pm} e_{j},
$$

valid for any $f^{ \pm} \in \stackrel{\mathcal{L}}{r}_{r}^{ \pm}$, has the required convergence properties.

\section{B.5 Expansion of $\Theta_{E}$ into Rank-One Mappings}

In this section we will decompose the map $\Theta_{E}: \mathfrak{A}(\mathcal{O}(r)) \rightarrow B(\mathcal{H})$, given by $\Theta_{E}(A)=$ $P_{E} A P_{E}$, as follows

$$
\Theta_{E}(A)=\hat{\Theta}_{E, 1}(A)+\stackrel{\vee}{\Theta}_{E, 1}(A)+\Theta_{E, 2}(A), \quad A \in \mathfrak{A}(\mathcal{O}) .
$$

Here $\hat{\Theta}_{E, 1}$ is a finite rank map, the part $\stackrel{\vee}{\Theta}_{E, 1}(A)$ collects the terms involving high derivatives of the field and $\Theta_{E, 2}(A)$ contains the contributions to $A$ coming from high Wick powers. In order to construct such a decomposition, we evaluate the map $\Theta_{E}$ on a Weyl operator $W(f), f \in \dot{\mathcal{L}}_{r}$, given by definition (B.2.10). We obtain the following expansion valid in the sense of quadratic forms on $D_{F} \times D_{F}$ :

$$
\begin{aligned}
\Theta_{E}(W(f)) & =e^{-\frac{1}{2}\|f\|_{2}^{2}} P_{E}: e^{i \sqrt{2} \phi_{+}\left(F^{+}\right)} e^{i \sqrt{2} \phi_{-}\left(F^{-}\right)}: P_{E} \\
& =e^{-\frac{1}{2}\|f\|_{2}^{2}} \sum_{k^{+}, k^{-} \in \mathbb{N}_{0}} \frac{(i \sqrt{2})^{k^{+}+k^{-}}}{k^{+} ! k^{-} !} P_{E}: \phi_{+}\left(F^{+}\right)^{k^{+}} \phi_{-}\left(F^{-}\right)^{k^{-}}: P_{E},
\end{aligned}
$$

where $\tilde{f}^{ \pm}=\omega^{\mp \frac{1}{2}} \tilde{F}^{ \pm}$. Now we introduce natural numbers $K^{+}, K^{-}$and the set of indices $\mathcal{S}=\left\{\left(k^{+}, k^{-}\right) \in \mathbb{N}_{0}^{2} \mid k^{+} \leq K^{+}\right.$and $\left.k^{-} \leq K^{-}\right\}$. We decompose the above sum into two parts

$$
\begin{aligned}
\Theta_{E}(W(f)) & =\Theta_{E, 1}(W(f))+\Theta_{E, 2}(W(f)) \\
\Theta_{E, 1}(W(f)) & =e^{-\frac{1}{2}\|f\|_{2}^{2}} \sum_{k^{+}, k^{-} \in \mathcal{S}} \frac{(i \sqrt{2})^{k^{+}+k^{-}}}{k^{+} ! k^{-} !} P_{E}: \phi_{+}\left(F^{+}\right)^{k^{+}} \phi_{-}\left(F^{-}\right)^{k^{-}}: P_{E}, \\
\Theta_{E, 2}(W(f)) & =e^{-\frac{1}{2}\|f\|_{2}^{2}} \sum_{k^{+}, k^{-} \in \mathcal{S}^{\prime}} \frac{(i \sqrt{2})^{k^{+}+k^{-}}}{k^{+} ! k^{-} !} P_{E}: \phi_{+}\left(F^{+}\right)^{k^{+}} \phi_{-}\left(F^{-}\right)^{k^{-}}: P_{E},
\end{aligned}
$$

where $\mathcal{S}^{\prime}$ is the complement of $\mathcal{S}$ and $\Theta_{E, 1}, \Theta_{E, 2}$ are understood as linear maps from the *-algebra $\mathfrak{A}(\mathcal{O}(r))$ of finite linear combinations of the Weyl operators, given by

$$
\mathfrak{\mathfrak { A }}(\mathcal{O}(r))=\operatorname{Span}\left\{W(f) \mid f \in \stackrel{\circ}{\mathcal{L}}_{r}\right\},
$$

to quadratic forms on $D_{F} \times D_{F}$. In the sequel we will show that $\Theta_{E, 1}$ and $\Theta_{E, 2}$ have their ranges in $B(\mathcal{H})$. Moreover, we will extend their domain, by continuity, to the whole local algebra $\mathfrak{A}(\mathcal{O}(r))$. For this purpose we will use the expansions of the functions $f^{ \pm} \in \dot{\mathcal{L}}_{r}^{ \pm}$ introduced in Section B.4. 


\section{B.5.1 Expansion of $\Theta_{E, 1}$}

First, recalling that $\tilde{F}_{E}^{ \pm}:=\chi_{E} \tilde{F}^{ \pm}$and exploiting the Wick ordering, we obtain

$$
P_{E}: \phi_{+}\left(F^{+}\right)^{k^{+}} \phi_{-}\left(F^{-}\right)^{k^{-}}: P_{E}=P_{E}: \phi_{+}\left(F_{E}^{+}\right)^{k^{+}} \phi_{-}\left(F_{E}^{-}\right)^{k^{-}}: P_{E} .
$$

With the help of the multinomial formula (B.1.4) and expansion (B.4.3) we get the following identity

$$
\begin{aligned}
& P_{E}: \phi_{+}\left(F_{E}^{+}\right)^{k^{+}} \phi_{-}\left(F_{E}^{-}\right)^{k^{-}}: P_{E} \\
& =\sum_{\substack{\alpha^{+}, \alpha^{-} \\
\left|\alpha^{ \pm}\right|=k^{ \pm}}} \frac{\left|\alpha^{+}\right| !|| \alpha^{-} \mid !}{\alpha^{+} ! \alpha^{-} !}\left\langle b_{\kappa, r}^{+} \mid f^{+}\right\rangle^{\alpha^{+}}\left\langle b_{\kappa, r}^{-} \mid f^{-}\right\rangle^{\alpha^{-}} P_{E}:\left(\partial \phi_{+}\right)^{\alpha^{+}}\left(\partial \phi_{-}\right)^{\alpha^{-}}: P_{E}
\end{aligned}
$$

where $\alpha^{ \pm}$are multiindices and we introduced the short-hand notation

$$
\left(\partial \phi_{ \pm}\right)^{\alpha^{ \pm}}=\prod_{j=1}^{\infty}\left(\partial^{\kappa_{j}} \phi_{ \pm}\right)^{\alpha^{ \pm}(j)}
$$

which refers to the numbering of the $s$-indices $\kappa$, introduced in formula (B.4.3). Identity (B.5.7) relies on the fact that the vectors $b_{\kappa, r}^{ \pm}$and $f^{ \pm}$are real in configuration space and exploits the relation

$$
\begin{aligned}
\left(\frac{1}{\sqrt{2}}\right)^{\left|\alpha^{+}\right|+\left|\alpha^{-}\right|} P_{E}:\left(a^{*}\left(\tilde{h}_{\kappa, E}^{+}\right)+a\left(\tilde{h}_{\kappa, E}^{+}\right)\right)^{\alpha^{+}} & \left(a^{*}\left(i \tilde{h}_{\kappa, E}^{-}\right)+a\left(i \tilde{h}_{\kappa, E}^{-}\right)\right)^{\alpha^{-}}: P_{E} \\
& =P_{E}:\left(\partial \phi_{+}\right)^{\alpha^{+}}\left(\partial \phi_{-}\right)^{\alpha^{-}}: P_{E}
\end{aligned}
$$

Substituting expansion (B.5.7) to (B.5.3), we obtain

$$
\begin{gathered}
\Theta_{E, 1}(W(f))=\sum_{\begin{array}{c}
\alpha^{+}, \alpha^{-} \\
\left(\left|\alpha^{+}\right|,\left|\alpha^{-}\right|\right) \in \mathcal{S}
\end{array}} \frac{(i \sqrt{2})^{\left|\alpha^{+}\right|+\left|\alpha^{-}\right|}}{\alpha^{+} ! \alpha^{-} !} e^{-\frac{1}{2}\|f\|_{2}^{2}}\left\langle b_{\kappa, r}^{+} \mid f^{+}\right\rangle^{\alpha^{+}}\left\langle b_{\kappa, r}^{-} \mid f^{-}\right\rangle^{\alpha^{-}} \\
\cdot P_{E}:\left(\partial \phi_{+}\right)^{\alpha^{+}}\left(\partial \phi_{-}\right)^{\alpha^{-}}: P_{E}
\end{gathered}
$$

Next, we choose some finite subset $\mathcal{M}$ of the set of all pairs of multiindices and decompose the map $\Theta_{E, 1}$ as follows

$$
\begin{aligned}
& \Theta_{E, 1}(W(f))=\hat{\Theta}_{E, 1}(W(f))+\stackrel{\vee}{\Theta}_{E, 1}(W(f)), \\
& \hat{\Theta}_{E, 1}(W(f))=e^{-\frac{1}{2}\|f\|_{2}^{2}} \sum_{\substack{\left(\alpha^{+}, \alpha^{-}\right) \in \mathcal{M} \\
\left(\left|\alpha^{+}\right|,\left|\alpha^{-}\right|\right) \in \mathcal{S}}} \frac{(i \sqrt{2})^{\left|\alpha^{+}\right|+\left|\alpha^{-}\right|}}{\alpha^{+} ! \alpha^{-} !}\left\langle b_{\kappa, r}^{+} \mid f^{+}\right\rangle^{\alpha^{+}}\left\langle b_{\kappa, r}^{-} \mid f^{-}\right\rangle^{\alpha^{-}} \\
& \text {- } P_{E}:\left(\partial \phi_{+}\right)^{\alpha^{+}}\left(\partial \phi_{-}\right)^{\alpha^{-}}: P_{E}, \\
& \stackrel{\vee}{\Theta}_{E, 1}(W(f))=e^{-\frac{1}{2}\|f\|_{2}^{2}} \sum_{\substack{\left(\alpha^{+}, \alpha^{-}\right) \in \mathcal{M}^{\prime} \\
\left(\left|\alpha^{+}\right|,\left|\alpha^{-}\right|\right) \in \mathcal{S}}} \frac{(i \sqrt{2})^{\left|\alpha^{+}\right|+\left|\alpha^{-}\right|}}{\alpha^{+} ! \alpha^{-} !}\left\langle b_{\kappa, r}^{+} \mid f^{+}\right\rangle^{\alpha^{+}}\left\langle b_{\kappa, r}^{-} \mid f^{-}\right\rangle^{\alpha^{-}} \\
& \cdot P_{E}:\left(\partial \phi_{+}\right)^{\alpha^{+}}\left(\partial \phi_{-}\right)^{\alpha^{-}}: P_{E} \cdot
\end{aligned}
$$


The above expressions can be restated in terms of suitable normal functionals on $B(\mathcal{H})$. We denote by $\bar{\sigma}_{\alpha^{+}, \alpha^{-}}^{(r)}$ the functionals from Proposition B.3.1, corresponding to the families of vectors $\left\{b_{\kappa_{j}, r}^{ \pm}\right\}_{j \in \mathbb{N}}$. Next, we define the normal functionals $\hat{\tau}_{\alpha^{+}, \alpha^{-}}^{(r)}$ on $B(\mathcal{H})$ given by

$$
\hat{\tau}_{\alpha^{+}, \alpha^{-}}^{(r)}=\frac{(i \sqrt{2})^{\left|\alpha^{+}\right|+\left|\alpha^{-}\right|}}{\alpha^{+} ! \alpha^{-} !} \bar{\sigma}_{\alpha^{+}, \alpha^{-}}^{(r)} .
$$

Making use of formula (B.5.12), we can write

$$
\hat{\Theta}_{E, 1}(A)=\sum_{\substack{\left(\alpha^{+}, \alpha^{-}\right) \in \mathcal{M} \\\left(\left|\alpha^{+}\right|,\left|\alpha^{-}\right|\right) \in \mathcal{S}}} \hat{\tau}_{\alpha^{+}, \alpha^{-}}^{(r)}(A) P_{E}:\left(\partial \phi_{+}\right)^{\alpha^{+}}\left(\partial \phi_{-}\right)^{\alpha^{-}}: P_{E}
$$

where $A \in \mathfrak{A}(\mathcal{O}(r))$ is any finite linear combination of Weyl operators. The Wick monomials : $\left(\partial \phi_{+}\right)^{\alpha^{+}}\left(\partial \phi_{-}\right)^{\alpha^{-}}$: belong to the field content of the theory (see definition (2.3.10) and relation (B.2.28) $)$, hence they are elements of $\mathcal{T}_{\infty}^{*}$. It follows that $\left\|P_{E}:\left(\partial \phi_{+}\right)^{\alpha^{+}}\left(\partial \phi_{-}\right)^{\alpha^{-}}: P_{E}\right\|<\infty$, thus $\hat{\Theta}_{E, 1}$ is a finite rank map from $\mathfrak{A}(\mathcal{O}(r))$ to $B(\mathcal{H})$. Since the functionals $\hat{\tau}_{\alpha^{+}, \alpha^{-}}^{(r)}$ are normal, $\hat{\Theta}_{E, 1}$ extends to a finite rank map from $\mathfrak{A}(\mathcal{O}(r))$ to $B(\mathcal{H})$.

In order to simplify expression (B.5.13) defining the map $\stackrel{\vee}{\Theta}_{E, 1}$, we note that for any two families of functions $\left\{F_{j}^{+}\right\}_{j \in \mathbb{N}},\left\{F_{j}^{-}\right\}_{j \in \mathbb{N}}$ from $S\left(\mathbb{R}^{s}\right)_{\mathbb{R}}$ and for any pair of multiindices $\left(\alpha^{+}, \alpha^{-}\right)$there holds the identity

$$
\begin{aligned}
2^{\frac{1}{2}\left|\alpha^{+}\right|} 2^{\frac{1}{2}\left|\alpha^{-}\right|}: & \phi_{+}\left(F^{+}\right)^{\alpha^{+}} \phi_{-}\left(F^{-}\right)^{\alpha^{-}}: \\
= & \sum_{\substack{\mu^{ \pm}, \nu^{ \pm} \\
\mu^{+}+\nu^{+}=\alpha^{+} \\
\mu^{-}+\nu^{-}=\alpha^{-}}} \frac{\alpha^{+} ! \alpha^{-} !}{\mu^{+} ! \nu^{+} ! \mu^{-} ! \nu^{-!}} a^{*}\left(\tilde{f}^{+}\right)^{\mu^{+}} a^{*}\left(i \tilde{f}^{-}\right)^{\mu^{-}} a\left(\tilde{f}^{+}\right)^{\nu^{+}} a\left(i \tilde{f}^{-}\right)^{\nu^{-}}
\end{aligned}
$$

where $\tilde{f}^{ \pm}=\omega^{\mp \frac{1}{2}} \tilde{F}^{ \pm}$and the equality holds in the sense of quadratic forms on $D_{F} \times D_{F}$. Consequently, we obtain from (B.5.13)

$$
\begin{aligned}
& \stackrel{\vee}{\Theta}_{E, 1}(W(f)) \\
& =\sum_{\substack{\bar{\mu}, \bar{\nu} \\
\mu+\bar{\nu} \in \mathcal{M}^{\prime} \\
\left(\left|\mu^{+}\right|+\left|\nu^{+}\right|,\left|\mu^{-}\right|+\left|\nu^{-}\right|\right) \in \mathcal{S}}} \frac{i^{\left|\mu^{+}\right|+\left|\nu^{+}\right|+2\left|\mu^{-}\right|}}{\bar{\mu} ! \bar{\nu} !} e^{-\frac{1}{2}\|f\|_{2}^{2}}\left\langle b_{\kappa, r} \mid f\right\rangle^{\bar{\mu}+\bar{\nu}} P_{E} a^{*}\left(h_{\kappa, E}\right)^{\bar{\mu}} a\left(h_{\kappa, E}\right)^{\bar{\nu}} P_{E} .
\end{aligned}
$$

We introduce the normal functionals $\stackrel{\vee}{\tau} \bar{\mu}, \bar{\nu}$ and the quadratic forms $\stackrel{\vee}{S}{ }_{\bar{\mu}, \bar{\nu}}$ on $D_{F} \times D_{F}$ given by

$$
\begin{aligned}
& \stackrel{\vee}{\tau} \bar{\mu}, \bar{\nu}_{(r)}^{(r)}:=\frac{i^{\left|\mu^{+}\right|+\left|\nu^{+}\right|+2\left|\mu^{-}\right|}}{\bar{\mu} ! \bar{\nu} !} \bar{\sigma}_{\bar{\mu}+\bar{\nu}}^{(r)}, \\
& \stackrel{\vee}{S}_{\bar{\mu}, \bar{\nu}}:=P_{E} a^{*}\left(h_{\kappa, E}\right)^{\bar{\mu}} a\left(h_{\kappa, E}\right)^{\bar{\nu}} P_{E} .
\end{aligned}
$$


Now expansion (B.5.17) takes the form

$$
\stackrel{\vee}{\Theta}_{E, 1}(A)=\sum_{\substack{\bar{\mu}, \bar{\nu} \\ \bar{\mu}+\bar{\nu} \in \mathcal{M}^{\prime} \\\left(\left|\mu^{+}\right|+\left|\nu^{+}\right|,\left|\mu^{-}\right|+\left|\nu^{-}\right|\right) \in \mathcal{S}}} \stackrel{\vee}{\tau}_{\bar{\mu}, \bar{\nu}}^{(r)}(A) \stackrel{\vee}{S}_{\bar{\mu}, \bar{\nu} .}
$$

In order to study its convergence properties we collect several auxiliary results. From Proposition B.3.1 we obtain the following lemma.

Lemma B.5.1. For any $l \geq 0$, the functionals $\stackrel{\vee}{\tau_{\bar{\mu}, \bar{\nu}}^{(r)}}$, defined by (B.5.18), satisfy the bound

$$
\left\|R^{-l} \tau_{\bar{\mu}, \bar{\nu}}^{\vee(r)} R^{-l}\right\| \leq\left(2 c_{l}\right)^{|\bar{\mu}|+|\bar{\nu}|} \sqrt{(|\bar{\mu}|+|\bar{\nu}|) !}\left\|b_{\kappa, r}\right\|_{2, l}^{\bar{\mu}+\bar{\nu}},
$$

where $c_{l}=\left(12+2 m^{2}\right)^{l / 2}$, and $m$ is the mass of the theory.

Next, to study the forms $\stackrel{\vee}{S}_{\bar{\mu}, \bar{\nu}}$, we recall the so-called energy bounds [BP90]:

Lemma B.5.2. For any $h_{1}, \ldots, h_{n} \in L^{2}\left(\mathbb{R}^{s}, d^{s} p\right)$ in the domain of $\omega^{\frac{1}{2}}$ there holds the bound

$$
\left\|a\left(\omega^{\frac{1}{2}} h_{1}\right) \ldots a\left(\omega^{\frac{1}{2}} h_{n}\right) P_{E}\right\| \leq E^{\frac{n}{2}}\left\|h_{1}\right\|_{2} \ldots\left\|h_{n}\right\|_{2} .
$$

Making use of the above result and Lemma B.4.4 we obtain that the forms $\stackrel{\vee}{S}_{\bar{\mu}, \bar{\nu}}$, defined by (B.5.19), are actually elements of $B(\mathcal{H})$ and satisfy the bound

$$
\left\|\stackrel{\vee}{S}_{\bar{\mu}, \bar{\nu}}\right\| \leq E^{\frac{|\bar{\mu}|+|\bar{\nu}|}{2}}\left\|\omega^{-\frac{1}{2}} \tilde{h}_{\kappa, E}\right\|_{2}^{\bar{\mu}+\bar{\nu}} .
$$

Now we are ready to prove the convergence of expansion (B.5.20).

Proposition B.5.3. Bos00] Let $\mathcal{S}=\left\{\left(k^{+}, k^{-}\right) \in \mathbb{N}_{0}^{2} \mid k^{+} \leq K^{+}\right.$and $\left.k^{-} \leq K^{-}\right\}$for some $K^{ \pm} \in \mathbb{N}_{0}$. Then, for any $0<p \leq 1$, there holds

$$
\sum_{\substack{\bar{\mu}, \bar{\nu} \\\left(\left|\mu^{+}\right|+\left|\nu^{+}\right|,\left|\mu^{-}\right|+\left|\nu^{-}\right|\right) \in \mathcal{S}}}\|\overbrace{\bar{\mu}, \bar{\nu}}^{\vee}\|^{p}\left\|\stackrel{\vee}{S}_{\bar{\mu}, \bar{\nu}}\right\|^{p}<\infty .
$$

Proof. From estimates (B.5.21), (B.5.23) we obtain

$$
\begin{aligned}
& \sum_{\substack{\bar{\mu}, \bar{\nu} \\
\left(\left|\mu^{+}\right|+\left|\nu^{+}\right|,\left|\mu^{-}\right|+\left|\nu^{-}\right|\right) \in \mathcal{S}}}\left\|\underset{\tau_{\bar{\mu}, \bar{\nu}}^{\vee}}{(r)}\right\|^{p}\left\|\stackrel{\vee}{S}_{\bar{\mu}, \bar{\nu}}\right\|^{p} \leq \\
& \leq\left(2^{K^{+}+K^{-}} \sqrt{\left(K^{+}+K^{-}\right) !}\right)^{p} \sum_{\substack{\bar{\mu}, \bar{\nu} \\
|\bar{\mu}|+|\bar{\nu}| \leq K^{+}+K^{-}}}\left(\left\|b_{\kappa, r}\right\|_{2}^{p}\left\|\omega^{-\frac{1}{2}} \tilde{h}_{\kappa, E}\right\|_{2}^{p}\right)^{\bar{\mu}+\bar{\nu}} .(\mathrm{B} .5 .)
\end{aligned}
$$

The sum on the r.h.s. can be estimated as follows

$$
\begin{aligned}
\left.\sum_{\substack{\mu, \bar{\nu} \\
|\bar{\mu}|+|\bar{\nu}| \leq K^{+}+K^{-}}}\left(\left\|b_{\kappa, r}\right\|_{2}^{p} \| \omega^{-\frac{1}{2}} \tilde{h}_{\kappa, E}\right) \|_{2}^{p}\right)^{\bar{\mu}+\bar{\nu}} \leq & \left(\sum_{k^{+}=0}^{M_{E}^{+}+M_{E}^{-}} \sum_{\substack{\mu^{+} \\
\left|\mu^{+}\right|=k^{+}}}\left(\left\|b_{\kappa, r}^{+}\right\|_{2}^{p}\left\|\omega^{-\frac{1}{2}} \tilde{h}_{\kappa, E}^{+}\right\|_{2}^{p}\right)^{\mu^{+}}\right)^{2} \\
& \cdot\left(\sum_{k^{-}=0}^{M_{E}^{+}+M_{E}^{-}} \sum_{\substack{\mu^{-} \\
\left|\mu^{-}\right|=k^{-}}}\left(\left\|b_{\kappa, r}^{-}\right\|_{2}^{p}\left\|\omega^{-\frac{1}{2}} \tilde{h}_{\kappa, E}^{-}\right\|_{2}^{p}\right)^{\mu^{-}}\right)^{2} .
\end{aligned}
$$


Making use of the fact that the multinomial coefficients are larger or equal to one and of the multinomial formula (B.1.4 $)$, we get

$$
\sum_{\substack{\mu^{+} \\\left|\mu^{+}\right|=k^{+}}}\left(\left\|b_{\kappa, r}^{+}\right\|_{2}^{p}\left\|\omega^{-\frac{1}{2}} \tilde{h}_{\kappa, E}^{+}\right\|_{2}^{p}\right)^{\mu^{+}} \leq\left(\sum_{\kappa \in \mathbb{N}_{0}^{s}}\left\|b_{\kappa, r}^{+}\right\|_{2}^{p}\left\|\omega^{-\frac{1}{2}} \tilde{h}_{\kappa, E}^{+}\right\|_{2}^{p}\right)^{k^{+}}
$$

and similarly for the sum w.r.t. $\mu^{-}$. The last expression is finite due to relation (B.4.17).

This proves the uniform convergence of expansion (B.5.17). It follows that the map $\stackrel{\vee}{\Theta}_{E, 1}$, which was defined on the norm dense subalgebra $\mathfrak{A}(\mathcal{O}(r))$, can be extended to the full local algebra $\mathfrak{A}(\mathcal{O}(r))$.

From definition (B.5.19) we obtain that in massive scalar free field theory of mass $m>0$ there holds $\stackrel{\vee}{S}_{\bar{\mu}, \bar{\nu}}=0$ for $|\bar{\mu}|>M_{E}$ or $|\bar{\nu}|>M_{E}$, where $M_{E}=\frac{E}{m}$. Thus, choosing $\mathcal{M}=\emptyset$ and $K^{+}=K^{-}=2\left[M_{E}\right]$, we obtain that $\Theta_{E}=\stackrel{\vee}{\Theta}_{E, 1}$. Hence Proposition $\mathbb{B} .5 .3$ and Lemma 3.5.1 give the known fact [BP90, Bos00] that Condition $N_{\sharp}$ holds in massive scalar free field theory.

Proposition B.5.4. In massive scalar free field theory there holds the identity

$$
\Theta_{E}(A)=\sum_{\bar{\mu}, \bar{\nu}} \underset{\tau}{\vee_{\bar{\mu}}(r)}(A) \stackrel{\vee}{S_{\bar{\mu}, \bar{\nu}}}, \quad A \in \mathfrak{A}(\mathcal{O}(r)),
$$

in the sense of norm convergence in $B(\mathcal{H})$. Moreover, for any $0<p \leq 1$ there holds the bound

$$
\sum_{\bar{\mu}, \bar{\nu}}\left\|\stackrel{\vee}{\tau_{\bar{\mu}}(r)} \bar{\nu}\right\|^{p}\left\|\stackrel{\vee}{S}_{\bar{\mu}, \bar{\nu}}\right\|^{p}<\infty
$$

However, the methods of the present subsection do not suffice to verify Condition $N_{\sharp}$ in massless free field theory. This goal is accomplished in the next subsection.

\section{B.5.2 Expansion of $\Theta_{E, 2}$}

Our last task is to complete the construction of the map $\Theta_{E, 2}$. In Subsection B.4.2 we introduced the $p$-nuclear positive operator $T$ which has a $J$-invariant orthonormal basis of eigenvectors $\left\{e_{i}\right\}_{1}^{\infty}$. We expand the functions $f^{ \pm} \in \stackrel{\circ}{\mathcal{L}}_{r}^{ \pm}$in this basis

$$
f^{ \pm}=\sum_{j=1}^{\infty}\left\langle e_{j} \mid f^{ \pm}\right\rangle e_{j}
$$

and make use of the multinomial formula $(\underline{B .1 .4})$, obtaining the following equality valid on $D_{F}$

$$
a^{(*)}\left(f^{ \pm}\right)^{m^{ \pm}}=\sum_{\mu^{ \pm},\left|\mu^{ \pm}\right|=m^{ \pm}} \frac{m^{ \pm} !}{\mu^{ \pm} !}\langle e \mid f\rangle^{\mu^{ \pm}} a^{(*)}\left(\mathcal{L}_{r}^{ \pm} e\right)^{\mu^{ \pm}} .
$$

Using relation (B.5.16), we obtain from definition (B.5.4) the following expansion, understood in the sense of quadratic forms on $D_{F} \times D_{F}$

$$
=\sum_{\substack{\bar{\mu}, \bar{\nu} \\\left(\left|\mu^{+}\right|+\left|\nu^{+}\right|,\left|\mu^{-}\right|+\left|\nu^{-}\right|\right) \in \mathcal{S}^{\prime}}}^{\Theta_{E, 2}(W(f))} \frac{i^{\left|\mu^{+}\right|+\left|\nu^{+}\right|+2\left|\mu^{-}\right|}}{\bar{\mu} ! \bar{\nu} !} e^{-\frac{1}{2}\|f\|_{2}^{2}}\langle e \mid f\rangle^{\bar{\mu}+\bar{\nu}} P_{E} a^{*}\left(\mathcal{L}_{r} e\right)^{\bar{\mu}} a\left(\mathcal{L}_{r} e\right)^{\bar{\nu}} P_{E} \cdot(
$$


From the second part of Proposition B.3.1 there follows the existence of normal functionals $\tau_{\bar{\mu}, \bar{\nu}}$ on $B(\mathcal{H})$ which have the property

$$
\tau_{\bar{\mu}, \bar{\nu}}(W(f))=\frac{i^{\left|\mu^{+}\right|+\left|\nu^{+}\right|+2\left|\mu^{-}\right|}}{\bar{\mu} ! \bar{\nu} !} e^{-\frac{1}{2}\|f\|_{2}^{2}}\langle e \mid f\rangle^{\bar{\mu}+\bar{\nu}}
$$

and satisfy the bound

$$
\left\|\tau_{\bar{\mu}, \bar{\nu}}\right\| \leq \frac{4^{|\bar{\mu}|+|\bar{\nu}|}}{(\bar{\mu} ! \bar{\nu} !)^{\frac{1}{2}}}\left(\frac{(\bar{\mu}+\bar{\nu}) !}{\bar{\mu} ! \bar{\nu} !}\right)^{\frac{1}{2}} \leq \frac{2^{\frac{5}{2}(|\bar{\mu}|+|\bar{\nu}|)}}{(\bar{\mu} ! \bar{\nu} !)^{\frac{1}{2}}}
$$

where we used properties of multinomial coefficients. Finally, we define the quadratic forms on $D_{F} \times D_{F}$ given by

$$
S_{\bar{\mu}, \bar{\nu}}=P_{E} a^{*}\left(\mathcal{L}_{r} e\right)^{\bar{\mu}} a\left(\mathcal{L}_{r} e\right)^{\bar{\nu}} P_{E} .
$$

We note that $a\left(\mathcal{L}_{r}^{ \pm} e\right)^{\bar{\nu}} P_{E}=a\left(Q_{E} \mathcal{L}_{r}^{ \pm} e\right)^{\bar{\nu}} P_{E}$ and $\omega^{-\frac{1}{2}} Q_{E} \mathcal{L}_{r}^{ \pm} e_{i}=T_{E, \pm} e_{i}$, where $T_{E, \pm}$ are bounded operators by Lemma B.4.5. Thus we obtain from the energy bounds (B.5.22) and definition (B.4.28) of the operator $T$

$$
\left\|S_{\bar{\mu}, \bar{\nu}}\right\| \leq E^{\frac{|\bar{\mu}|+|\bar{\nu}|}{2}}\left\|\omega^{-\frac{1}{2}} Q_{E} \mathcal{L}_{r} e\right\|_{2}^{\bar{\mu}}\left\|\omega^{-\frac{1}{2}} Q_{E} \mathcal{L}_{r} e\right\|_{2}^{\bar{\nu}} \leq E^{\frac{|\overline{\mid}|+|\bar{\nu}|}{2}} t^{\bar{\mu}} t^{\bar{\nu}}
$$

where $\left\{t_{j}\right\}_{1}^{\infty}$ are eigenvalues of $T$. We have arrived at the following expansion, still in the sense of quadratic forms on $D_{F} \times D_{F}$

$$
\Theta_{E, 2}(A)=\sum_{\substack{\bar{\mu}, \bar{\nu} \\\left(\left|\mu^{+}\right|+\left|\nu^{+}\right|,\left|\mu^{-}\right|+\left|\nu^{-}\right|\right) \in \mathcal{S}^{\prime}}} \tau_{\bar{\mu}, \bar{\nu}}(A) S_{\bar{\mu}, \bar{\nu}}
$$

valid for any $A \in \mathfrak{A}(\mathcal{O}(r))$ i.e. for any finite, linear combination of Weyl operators. Our task is to establish the convergence of this sum in the norm topology of $B(\mathcal{H})$ and extend this map by continuity to all $A \in \mathfrak{A}(\mathcal{O}(r))$. It suffices to consider the case $\mathcal{S}=\{0,0\}$ when there holds

$$
\Theta_{E}(A)=\omega_{0}(A) P_{E}+\Theta_{E, 2}(A), \quad A \in \mathfrak{A}(\mathcal{O}) .
$$

The following proposition verifies the known fact that Condition $N_{\sharp}$ holds in scalar free field theory.

Proposition B.5.5. [BP90, Bos00] In massive and massless scalar free field theory there holds the identity

$$
\Theta_{E}(A)=\sum_{\bar{\mu}, \bar{\nu}} \tau_{\bar{\mu}, \bar{\nu}}(A) S_{\bar{\mu}, \bar{\nu}}, \quad A \in \mathfrak{A}(\mathcal{O}(r)),
$$

in the sense of norm convergence in $B(\mathcal{H})$. Moreover, for any $0<p \leq 1$ there holds the bound

$$
\sum_{\bar{\mu}, \bar{\nu}}\left\|\tau_{\bar{\mu}, \bar{\nu}}\right\|^{p}\left\|S_{\bar{\mu}, \bar{\nu}}\right\|^{p}<\infty
$$


Proof. We note the following estimate

$$
\begin{aligned}
\sum_{\bar{\mu}, \bar{\nu}}\left\|\tau_{\bar{\mu}, \bar{\nu}}\right\|^{p}\left\|S_{\bar{\mu}, \bar{\nu}}\right\|^{p} & \leq \sum_{\bar{\mu}, \bar{\nu}} \frac{\left(2^{5} E\right)^{\frac{1}{2} p(|\bar{\mu}|+|\bar{\nu}|)}}{(\bar{\mu} !)^{\frac{1}{2} p}(\bar{\nu} !)^{\frac{1}{2} p}} t^{p \bar{\mu}} t^{p \bar{\nu}} \\
& \leq \sum_{m^{ \pm}, n^{ \pm} \in \mathbb{N}_{0}} \frac{\left.\left(2^{5 / 2} E^{1 / 2}\|T\|_{p}\right)^{p\left(m^{+}+m^{-}+n^{+}+n^{-}\right.}\right)}{\left(m^{+} ! m^{-} ! n^{+} ! n^{-} !\right)^{\frac{1}{2} p}}
\end{aligned}
$$

where in the first step we used (B.5.34) and (B.5.36). In the second step we made use of the fact that the multinomial coefficients are larger or equal to one, of the identity

$$
\sum_{\mu^{ \pm},\left|\mu^{ \pm}\right|=m^{ \pm}} \frac{m^{ \pm} !}{\mu^{ \pm !}}\left(t^{p}\right)^{\mu^{ \pm}}=\left(\|T\|_{p}^{p}\right)^{m^{ \pm}}
$$

and its counterpart for the sums w.r.t. $\nu^{ \pm}$.

Thus the map $\Theta_{E, 2}$, given by (B.5.37), has its range in $B(\mathcal{H})$ and extends by continuity to the whole local algebra $\mathfrak{A}(\mathcal{O}(r))$. We denote the resulting map by the same symbol. 


\section{Appendix C}

\section{Verification of Condition $L^{(2)}$ in Scalar Free Field Theory}

The goal of this appendix is to verify that Condition $L^{(2)}$, introduced in Section 2.2, holds in scalar free field theory. In the massive case this fact follows from Condition $L^{(1)}$, verified in Appendix [D] and Theorem 2.3.1. Thus our main interest in the present appendix is in massless scalar free field theory, although some results will be stated for general $m \geq 0$ to facilitate their application in other contexts. Our aim is to prove Theorems 2.2.5 2.2.6 and 2.2.7 which are at the basis of our discussion in Subsection 2.2.3

The proofs of these three statements are given in Section C.1. They rely on the auxiliary Theorem C.1.1 stated below, whose proof is the subject of the later part of this appendix. In Section C.2 we define the functionals $\tau_{k}, k \in\{1,2,3\}$, on $\hat{\mathfrak{A}}$ and verify that they have the properties required in the statement of Theorem C.1.1 We also show that the map $R^{(2)}$, defined in Theorem C.1.1 can be expressed in terms of the maps $\stackrel{\vee}{\Theta}_{E, 1}$ and $\Theta_{E, 2}$, introduced in Appendix B. In Section C.3 we show that the range of $R^{(2)}$ consists of square-integrable operators. The argument is divided into three parts: In Subsection C.3.1 we prove a variant of Theorem 1.6.1 which is applicable to the present problem. In Subsections C.3.2 and C.3.3 we apply this result to prove the square-integrability of the ranges of the maps $\stackrel{\vee}{\Theta}_{E, 1}$ and $\Theta_{E, 2}$, respectively.

\section{C.1 Proofs of Theorems 2.2.5, 2.2.6 and 2.2.7 based on The- orem C.1.1}

Our discussion in this section is based on the following theorem, whose proof is given in Sections C.2 and C.3

Theorem C.1.1. In massless scalar free field theory in $s \geq 3$ dimensional space there exist linear functionals $\tau_{1}, \tau_{2}, \tau_{3}$ on $\hat{\mathfrak{A}}$, invariant under translations in space, s.t. for any $A \in \hat{\mathfrak{A}}$ the quantity

$$
R^{(2)}(A):=A-\omega_{0}(A) I-\tau_{1}(A) \phi_{+}-\tau_{2}(A): \phi_{+}^{2}:-\tau_{3}(A): \phi_{+}^{3}:,
$$

defined as a quadratic form on the domain $D_{B} \times D_{B}$ of vectors of bounded energy, satisfies $\left\|R^{(2)}(A)\right\|_{E, 2}<\infty$ for any $E \geq 0$. Moreover, $\omega_{0}, \tau_{1}, \tau_{2}, \tau_{3}$ form a linearly independent 
family. In addition, there hold the following assertions:

$$
\begin{aligned}
& \hat{\mathfrak{A}}^{(e)} \subset \operatorname{ker} \tau_{1} \cap \operatorname{ker} \tau_{3} \text { and }\left.\tau_{2}\right|_{\hat{\mathfrak{A}}^{(e)}} \neq 0, \\
& \hat{\mathfrak{A}}^{(d)} \subset \operatorname{ker} \tau_{1} \cap \operatorname{ker} \tau_{2} \cap \operatorname{ker} \tau_{3} .
\end{aligned}
$$

It is an immediate consequence of this theorem that the theory $\left(\mathfrak{A}^{(\mathrm{d})}, \alpha, \mathcal{H}\right)$, generated by the derivatives of the massless scalar free field, satisfies Condition $L^{(2)}$ and has trivial point-continuous subspace. In fact:

Proof of Theorem 2.2.7; The statement follows directly from relations (C.1.3) and (C.1.1).

Let us now consider the full massless scalar free field theory $(\mathfrak{A}, \alpha, \mathcal{H})$. Theorem C.1.1 reduces the analysis of the point-continuous subspace in this model to the study of the three pointlike-localized fields: $\phi_{+},: \phi_{+}^{2}:,: \phi_{+}^{3}:$. We will show below that the concepts of square-integrability and of the infrared order of an operator, defined for observables from $\hat{\mathfrak{A}}_{\mathrm{c}}$ by relations (2.2.17) and (2.2.22), respectively, can be extended to the fields in question. Moreover, there holds the following proposition, whose proof is given in the later part of this section.

Proposition C.1.2. In massless scalar free field theory the following statements hold true:

(a) If $s \geq 3$, then $\operatorname{ord}\left(\phi_{+}\right)=2$.

(b) If $s=3$, then $\operatorname{ord}\left(: \phi_{+}^{2}:\right)=1$.

If $s=4$, then $\operatorname{ord}\left(: \phi_{+}^{2}:\right)=0$.

If $s \geq 5$, then $\left\|: \phi^{2}:\right\|_{E, 2}<\infty$ for any $E \geq 0$.

(c) If $s=3$, then $\operatorname{ord}\left(: \phi_{+}^{3}:\right)=0$.

If $s \geq 3$, then $\left\|: \phi^{3}:\right\|_{E, 2}<\infty$ for any $E \geq 0$.

Part (b) also holds if $: \phi_{+}^{2}:$ is replaced with $: \phi_{+}^{2}: \in \Phi_{\mathrm{FH}}^{(e)}$.

We note that vanishing of the infrared order does not imply that a given operator is square-integrable. There remains an open question if $: \phi_{+}^{2}:$ for $s=4$ and $: \phi_{+}^{3}:$ for $s=3$ have the property of square-integrability. Its resolution would allow one to determine exactly the dimensions of the point-continuous subspaces in Theorem 2.2.5 (a) and (b), and Theorem 2.2.6 (b).

After this preparation we estimate the dimension of the point-continuous subspace in (full) massless scalar free field theory and compute the infrared orders of its elements. As expected, the infrared structure improves with increasing dimension of space, in the sense that the dimension of the point-continuous subspace decreases. However, this subspace remains non-trivial for any $s \geq 3$.

Proof of Theorem 2.2.5 We consider only the case $s=3$ as the remaining cases can be proven analogously. Since we do not know whether : $\phi_{+}^{3}:$, whose infrared order is zero by Proposition C.1.2, is also square-integrable, we have to consider both possibilities. First, we show that if $\left\|: \phi_{+}^{3}:\right\|_{E, 2}=\infty$ for some $E \geq 0$, then for any $A \in \hat{\mathfrak{A}}_{\mathrm{c}}$ there hold the following statements:

(i) $\tau_{1}(A) \neq 0 \Leftrightarrow \operatorname{ord}(A)=2$.

(ii) $\left(\tau_{1}(A)=0\right.$ and $\left.\tau_{2}(A) \neq 0\right) \Leftrightarrow \operatorname{ord}(A)=1$. 
(iii) $\left(\tau_{1}(A)=0, \tau_{2}(A)=0\right.$ and $\left.\tau_{3}(A) \neq 0\right) \Leftrightarrow\left(\operatorname{ord}(A)=0\right.$ and $\exists_{E \geq 0}$ s.t. $\left.\|A\|_{E, 2}=\infty\right)$.

(iv) $\left(\tau_{1}(A)=0, \tau_{2}(A)=0\right.$ and $\left.\tau_{3}(A)=0\right) \Leftrightarrow \forall_{E \geq 0}\|A\|_{E, 2}<\infty$.

To justify these claims, suppose that $\tau_{0}(A)=\cdots=\tau_{l-1}(A)=0$ for some $l \in\{1,2,3,4\}$. $\left(\tau_{0}:=\omega_{0}\right.$ is understood here). Then relation (C.1.1) gives the following bounds for any $\varphi \in \mathcal{T}_{E, 1}$

$$
\begin{aligned}
& \pm\left\{\left(\int d^{s} p|\vec{p}|^{\beta}|\varphi(\widetilde{A}(\vec{p}))|^{2}\right)^{\frac{1}{2}}-\left|\tau_{l}(A)\right|\left(\int d^{s} p|\vec{p}|^{\beta}\left|\varphi\left(\widetilde{: \phi_{+}^{l}}:(\vec{p})\right)\right|^{2}\right)^{\frac{1}{2}}\right\} \\
\leq & \sum_{k=l+1}^{3}\left|\tau_{k}(A)\right| \sup _{\varphi^{\prime} \in \mathcal{T}_{E, 1}}\left(\int d^{s} p|\vec{p}|^{\beta}\left|\varphi^{\prime}\left(\widetilde{: \phi_{+}^{k}}:(\vec{p})\right)\right|^{2}\right)^{\frac{1}{2}}+E^{\frac{\beta}{2}}\left\|R^{(2)}(A)\right\|_{E, 2} .
\end{aligned}
$$

We note that all the terms in this expression are finite for sufficiently large $\beta$ by estimate (2.2.15) and Proposition C.1.2. We will now study their behavior with decreasing $\beta$. By Proposition C.1.2 ord $\left(: \phi_{+}^{l}:\right)>\operatorname{ord}\left(: \phi_{+}^{k}:\right)$, for $k>l$ in the above formula. Thus, by considering $\beta$ in a small neighborhood of ord $\left(: \phi_{+}^{l}:\right)$ and taking supremum w.r.t. $\varphi \in \mathcal{T}_{E, 1}$, we easily obtain that $\operatorname{ord}(A)=\operatorname{ord}\left(: \phi_{+}^{l}:\right)$ if and only if $\tau_{l}(A) \neq 0$. Hence, there holds (i) and (ii). In part (iii) we set $\beta=0$ and make use of our assumption that $\left\|: \phi_{+}^{3}:\right\|_{E, 2}=\infty$ for some $E \geq 0$. In (iv) the implication $(\Rightarrow$ ) follows trivially from the square-integrability of $R^{(2)}(A)$. The opposite implication is a consequence of (i), (ii) and (iii).

Thus we have verified that $\operatorname{Ord}\left(\hat{\mathfrak{A}}_{\mathrm{c}}\right)=\{0,1,2\}$ and the subspace $\hat{\mathfrak{A}}_{\text {ac }}$, consisting of the square-integrable observables, can be expressed as follows

$$
\hat{\mathfrak{A}}_{\mathrm{ac}}=\operatorname{ker} \omega_{0} \cap \operatorname{ker} \tau_{1} \cap \operatorname{ker} \tau_{2} \cap \operatorname{ker} \tau_{3},
$$

Now computation of the dimension of the point-continuous subspace is a simple exercise in linear algebra: Since the above functionals are linearly independent (by Theorem C.1.1), we can find $A_{1}, A_{2}, A_{3} \in \hat{\mathfrak{A}}_{\mathrm{c}}$ s.t. $\tau_{i}\left(A_{j}\right)=\delta_{i, j}$. For any $A \in \hat{\mathfrak{A}}_{\mathrm{c}}$ we obtain the decomposition

$$
A=\left(A-A_{1} \tau_{1}(A)-A_{2} \tau_{2}(A)-A_{3} \tau_{3}(A)\right)+A_{1} \tau_{1}(A)+A_{2} \tau_{2}(A)+A_{3} \tau_{3}(A),
$$

where the term in bracket belongs to $\hat{\mathfrak{A}}_{\mathrm{ac}}$ due to (C.1.5). Choosing the point-continuous subspace as $\hat{\mathfrak{A}}_{\mathrm{pc}}=\operatorname{Span}\left\{A_{1}, A_{2}, A_{3}\right\}$ and noting that $\left\{A_{j}\right\}_{1}^{3}$ are linearly independent, we obtain that $\operatorname{dim} \hat{\mathfrak{A}}_{\mathrm{pc}}=3$.

Assuming that for any $E \geq 0$ there holds $\left\|: \phi_{+}^{3}:\right\|_{E, 2}<\infty$, we can incorporate the term $\tau_{3}(\cdot): \phi_{+}^{3}$ : to $R^{(2)}(\cdot)$ in formula (C.1.1). Thus, proceeding analogously as in the previous case, we verify the following facts for any $A \in \hat{\mathfrak{A}}_{\mathrm{c}}$ :

$\left(\mathrm{i}^{\prime}\right) \tau_{1}(A) \neq 0 \Leftrightarrow \operatorname{ord}(A)=2$.

(ii') $\left(\tau_{1}(A)=0\right.$ and $\left.\tau_{2}(A) \neq 0\right) \Leftrightarrow \operatorname{ord}(A)=1$.

(iii') $\left(\tau_{1}(A)=0\right.$ and $\left.\tau_{2}(A)=0\right) \Leftrightarrow\left(\forall_{E \geq 0}\|A\|_{E, 2} \leq \infty\right)$.

Again, it follows that $\operatorname{Ord}\left(\hat{\mathfrak{A}}_{\mathrm{c}}\right)=\{0,1,2\}$. However, the absolutely continuous subspace is now given by

$$
\hat{\mathfrak{A}}_{\mathrm{ac}}=\operatorname{ker} \omega_{0} \cap \operatorname{ker} \tau_{1} \cap \operatorname{ker} \tau_{2} .
$$

Hence the point-continuous subspace is two-dimensional in this case. In view of relations (C.1.5) and (C.1.7) the theory satisfies Condition $L^{(2)}$. 
The last example which we consider is the even part of massless scalar free field theory. Making use of Theorem C.1.1 we will show that the theory $\left(\underline{\mathfrak{A}}^{(\mathrm{e})}, \underline{\alpha}, \mathcal{H}^{(\mathrm{e})}\right)$, introduced in Section B.2. satisfies Condition $L^{(2)}$ and we will analyze the resulting point-continuous subspace. To this end, we define the functionals $\underline{\omega}_{0}=\omega_{0} \circ \pi_{(\mathrm{e})}^{-1}, \underline{\tau}_{2}=\tau_{2} \circ \pi_{(\mathrm{e})}^{-1}$ and set for any $\underline{A} \in \underline{\mathfrak{A}}^{(\mathrm{e})}$

$$
R_{(\mathrm{e})}^{(2)}(\underline{A}):=\underline{A}-\underline{\omega}_{0}(\underline{A}) \underline{I}-\underline{\tau}_{2}(\underline{A}): \underline{\phi_{+}^{2}}:
$$

as a quadratic form on states of bounded energy in $\mathcal{H}^{(\mathrm{e})}$. Then, due to relation (B.2.18) and the fact that $\underline{\varphi}\left(: \phi_{+}^{2}:\right)=\iota_{(\mathrm{e})}(\underline{\varphi})\left(: \phi_{+}^{2}:\right)$ for any $\underline{\varphi} \in \mathcal{T}_{E}^{(\mathrm{e})}$, there holds

$$
\underline{\varphi}\left(R_{(\mathrm{e})}^{(2)}(\underline{A})\right)=\iota_{(\mathrm{e})}(\underline{\varphi})\left(R^{(2)}\left(\pi_{(\mathrm{e})}^{-1}(\underline{A})\right)\right), \quad \underline{A} \in \underline{\mathfrak{A}}^{(\mathrm{e})}, \underline{\varphi} \in \mathcal{T}_{E}^{(\mathrm{e})} .
$$

Finally, making use of relation $(\underline{B .2 .20})$, we obtain $\left\|R_{(\mathrm{e})}^{(2)}(\underline{A})\right\|_{E, 2} \leq\left\|R^{(2)}\left(\pi_{(\mathrm{e})}^{-1}(\underline{A})\right)\right\|_{E, 2}<$ $\infty$, where the last bound follows from Theorem C.1.1. With the help of Proposition C.1.2 we obtain the description of the point-continuous subspace in the even part of massless scalar free field theory. Here the only possible obstruction to the square-integrability of observables form $\hat{\mathfrak{A}}_{\mathrm{c}}^{(\mathrm{e})}$ is the presence of the term : $\phi_{+}^{2}$ : in relation (C.1.8).

Proof of Theorem 2.2.6: Exploiting relation (C.1.8) and the subsequent discussion, and proceeding as in the proof of Theorem 2.2.5 above, we obtain the result.

The remaining part of this section is devoted to the proof of Proposition C.1.2 which was the main technical input of the above discussion. We note that any field $\phi \in \Phi_{\mathrm{FH}}$ belongs to $\mathcal{T}_{\infty}^{*}$ and therefore $\varphi(\phi(\vec{x}))$ is a bounded, continuous function for any $\varphi \in \mathcal{T}_{E}$. We are interested in the regularity properties of its Fourier transform $\varphi(\widetilde{\phi}(\vec{p}))$ which is a tempered distribution. To begin with, we prove a simple, technical lemma.

Lemma C.1.3. Let $0<\beta<s, \phi \in \mathcal{T}_{E}^{*}$ and $D \subset \mathcal{H}$ be a domain s.t. $P_{E} \mathcal{H} \cap D$ is dense in $P_{E} \mathcal{H}$ and $|\vec{p}|^{\frac{\beta}{2}}\left(\Psi_{1} \mid \widetilde{\phi}(\vec{p}) \Psi_{2}\right)$ is square-integrable, uniformly in $\Psi_{1}, \Psi_{2} \in\left(P_{E} \mathcal{H} \cap D\right)_{1}$. Then $|\vec{p}|^{\frac{\beta}{2}} \varphi(\widetilde{\phi}(\vec{p}))$ is square-integrable, uniformly in $\varphi \in \mathcal{T}_{E, 1}$, and

$$
\sup _{\Psi_{1}, \Psi_{2} \in\left(P_{E} \mathcal{H} \cap D\right)_{1}} \int d^{s} p|\vec{p}|^{\beta}\left|\left(\Psi_{1} \mid \widetilde{\phi}(\vec{p}) \Psi_{2}\right)\right|^{2}=\sup _{\varphi \in \mathcal{T}_{E, 1}} \int d^{s} p|\vec{p}|^{\beta}|\varphi(\widetilde{\phi}(\vec{p}))|^{2} .
$$

Proof. By the Cauchy-Schwarz inequality, there holds for any $g \in S\left(\mathbb{R}^{s}\right)$ and $\Psi_{1}, \Psi_{2} \in$ $\left(P_{E} \mathcal{H} \cap D\right)$

$$
\begin{aligned}
& \left|\left(\Psi_{1} \mid \phi(g) \Psi_{2}\right)\right| \\
& \leq\left\|\Psi_{1}\right\|\left\|\Psi_{2}\right\| \sup _{\Psi^{\prime}{ }_{1}, \Psi^{\prime}{ }_{2} \in\left(P_{E} \mathcal{H} \cap D\right)_{1}}\left(\int d^{s} p|\vec{p}|^{\beta}\left|\left(\Psi^{\prime}{ }_{1} \mid \widetilde{\phi}(\vec{p}) \Psi^{\prime}{ }_{2}\right)\right|^{2}\right)^{\frac{1}{2}}\left(\int d^{s} p \frac{1}{|\vec{p}|^{\beta}}|\tilde{g}(\vec{p})|^{2}\right)^{\frac{1}{2}} \cdot(\mathrm{C})
\end{aligned}
$$

The above bound extends, by continuity, to any $\Psi_{1}, \Psi_{2} \in P_{E} \mathcal{H}$ and we can proceed as in the proof of Theorem 2.5 from [Bu90]: Let $L^{2}\left(\mathbb{R}^{s}\right)_{(\beta)}$ be the Hilbert space of (classes of) functions $h$ on $\mathbb{R}^{s}$ for which

$$
\|h\|_{2,(\beta)}^{2}=\int d^{s} p|\vec{p}|^{-\beta}|h(\vec{p})|^{2}<\infty .
$$


Clearly, the subspace of test functions from $S\left(\mathbb{R}^{s}\right)$ is dense in $L^{2}\left(\mathbb{R}^{s}\right)_{(\beta)}$. It follows from relation (C.1.11) that for any $\varphi \in \mathcal{T}_{E, 1}$

$$
\begin{aligned}
& |\varphi(\phi(g))| \\
& \leq\left\|P_{E} \phi(g) P_{E}\right\| \leq\|g\|_{2,(\beta)} \sup _{\Psi^{\prime}{ }_{1}, \Psi^{\prime}{ }_{2} \in\left(P_{E} \mathcal{H} \cap D\right)_{1}}\left(\int d^{s} p|\vec{p}|^{\beta} \mid\left(\Psi^{\prime}{ }_{1} \mid \widetilde{\phi}(\vec{p}) \Psi^{\prime}{ }_{2}\right)^{2}\right)^{2} .
\end{aligned}
$$

The above inequality and the Riesz theorem imply that $\varphi(\widetilde{\phi}(\vec{p})) \in L^{2}\left(\mathbb{R}^{s}\right)_{(-\beta)}$ and

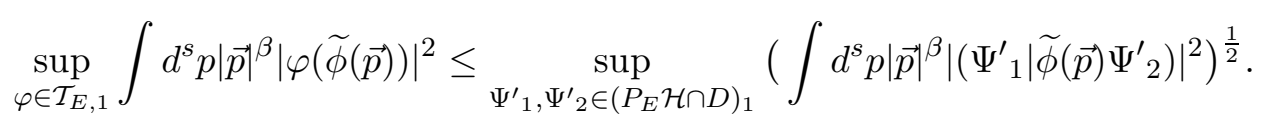

The opposite inequality is trivial, since the supremum on the r.h.s extends over a smaller set.

Setting in the above lemma $\phi=\phi_{+}$and $D=D_{S}$, we obtain a prescription for computation of the infrared order of the field $\phi_{+}$:

Proof of Proposition C.1.2 (a): We will establish the bound

$$
\sup _{\varphi \in \mathcal{T}_{E, 1}} \int d^{s} p|\vec{p}|^{2}\left|\varphi\left(\widetilde{\phi}_{+}(\vec{p})\right)\right|^{2}<\infty
$$

and show that the power of the mollifier $|\vec{p}|^{2}$ cannot be reduced. By Lemma C.1.3, it suffices to consider $\varphi(\cdot)=\left(\Psi_{1} \mid \cdot \Psi_{2}\right)$, where $\Psi_{1}, \Psi_{2} \in D_{S} \cap P_{E} \mathcal{H}$. Making use of the fact that

$$
\widetilde{\phi}_{+}(\vec{p})=\frac{1}{\sqrt{2|\vec{p}|}}\left(a^{*}(\vec{p})+a(-\vec{p})\right),
$$

in the sense of quadratic forms on $D_{S} \times D_{S}$, we obtain the estimate

$$
\begin{aligned}
\int d^{s} p|\vec{p}|^{2} & \left.\left(\Psi_{1} \mid \widetilde{\phi}_{+}(\vec{p}) \Psi_{2}\right)\right|^{2} \\
& \leq \int d^{s} p|\vec{p}|\left(\left\|\Psi_{1}\right\|^{2}\left(\Psi_{2} \mid a^{*}(\vec{p}) a(\vec{p}) \Psi_{2}\right)+\left\|\Psi_{2}\right\|^{2}\left(\Psi_{1} \mid a^{*}(\vec{p}) a(\vec{p}) \Psi_{1}\right)\right) \\
& \leq 2 E\left\|\Psi_{1}\right\|^{2}\left\|\Psi_{2}\right\|^{2}
\end{aligned}
$$

where we used representation $(\mathbb{B} .2 .23)$ of the Hamiltonian. To show that the bound for $\phi_{+}$is optimal, we construct a suitable sequence of functionals: We choose a positive function $h(\vec{p}) \in C_{0}^{\infty}\left(\mathbb{R}^{s}\right)$ s.t. $\operatorname{supp} h(\vec{p}) \subset\left\{\vec{p} \in \mathbb{R}^{s}|| \vec{p} \mid \leq E\right\}, \int|h(\vec{p})|^{2} d^{s} p=1$ and set $H_{n}(\vec{p})=n^{\frac{s}{2}} h(n \vec{p})$ for $n \in \mathbb{N}$. Then the functionals on $B(\overline{\mathcal{H}})$, given by

$$
\varphi_{n}^{(1)}(\cdot)=\left(H_{n}^{\otimes(n-1)} \mid \cdot H_{n}^{\otimes n}\right),
$$

clearly belong to $\mathcal{T}_{E, 1}$. We note that for any $\varepsilon>0$

$$
\begin{aligned}
\int d^{s} p|\vec{p}|^{2-\varepsilon}\left|\varphi_{n}^{(1)}\left(\widetilde{\phi}_{+}(\vec{p})\right)\right|^{2} & =\frac{1}{2} \int d^{s} p|\vec{p}|^{1-\varepsilon}\left|\left(H_{n}^{\otimes(n-1)} \mid a(\vec{p}) H_{n}^{\otimes n}\right)\right|^{2} \\
& =\frac{n^{\varepsilon}}{2} \int d^{s} p|\vec{p}|^{1-\varepsilon}|h(\vec{p})|^{2} .
\end{aligned}
$$

By taking the limit $n \rightarrow \infty$, the claim follows.

In order to compute the infrared orders of higher Wick powers of $\phi_{+}$, we need the following lemma. 
Lemma C.1.4. Let $\Psi_{1}, \Psi_{2}$ be normalized vectors from $D_{S} \cap P_{E} \mathcal{H}$. Then

$$
\int d^{s} p_{1} \ldots d^{s} p_{n}\left|\vec{p}_{1}\right|^{2} \ldots\left|\vec{p}_{n}\right|^{2}\left|\left(\Psi_{1} \mid: \widetilde{\phi}_{+}\left(\vec{p}_{1}\right) \ldots \widetilde{\phi}_{+}\left(\vec{p}_{n}\right): \Psi_{2}\right)\right|^{2} \leq c_{n, s, E}
$$

for some constant $c_{n, s, E}$ independent of $\Psi_{1}, \Psi_{2}$.

Proof. Due to formula (C.1.16), it is clear that the expression on the l.h.s. of (C.1.20) can be bounded by a linear combination of terms of the form

$$
\begin{aligned}
& \int d^{s} p_{1} \ldots d^{s} p_{n}\left|\vec{p}_{1}\right| \ldots\left|\vec{p}_{n}\right|\left|\left(\Psi_{1} \mid a^{*}\left(\vec{p}_{1}\right) \ldots a^{*}\left(\vec{p}_{k}\right) a\left(\vec{p}_{k+1}\right) \ldots a\left(\vec{p}_{n}\right) \Psi_{2}\right)\right|^{2} \\
& \leq \int d^{s} p_{1} \ldots d^{s} p_{k}\left|\vec{p}_{1}\right| \ldots\left|\vec{p}_{k}\right|\left(\Psi_{1} \mid a^{*}\left(\vec{p}_{1}\right) \ldots a^{*}\left(\vec{p}_{k}\right) a\left(\vec{p}_{k}\right) \ldots a\left(\vec{p}_{1}\right) \Psi_{1}\right) \\
& \cdot \int d^{s} p_{k+1} \ldots d^{s} p_{n}\left|\vec{p}_{k+1}\right| \ldots\left|\vec{p}_{n}\right|\left(\Psi_{2} \mid a^{*}\left(\vec{p}_{k+1}\right) \ldots a^{*}\left(\vec{p}_{n}\right) a\left(\vec{p}_{n}\right) \ldots a\left(\vec{p}_{k+1}\right) \Psi_{2}\right) \leq E^{n},
\end{aligned}
$$

where in the first step we made use of the Cauchy-Schwarz inequality and in the second step of the representation (B.2.23) of the Hamiltonian.

After this preparation we turn to the Wick powers of the field $\phi_{+}$.

Lemma C.1.5. Let $n>1, s \geq 3$. Then, for any $\beta \geq 0$ s.t. $\beta>2-(s-2)(n-1)$, there holds the bound

$$
\sup _{\varphi \in \mathcal{T}_{E, 1}} \int d^{s} p|\vec{p}|^{\beta}\left|\varphi\left(: \widetilde{\phi}_{+}^{n}:(\vec{p})\right)\right|^{2}<\infty .
$$

Proof. We fix $\Psi_{1}, \Psi_{2} \in\left(D_{S} \cap P_{E} \mathcal{H}\right)_{1}$ and define the functions

$$
\begin{aligned}
F_{n}(\vec{p})=\int d^{s} q_{1} \ldots d^{s} q_{n-1}\left|\vec{p}-\vec{q}_{1}\right|^{2}\left|\vec{q}_{1}-\vec{q}_{2}\right|^{2} \ldots\left|\vec{q}_{n-1}\right|^{2} \\
\cdot\left|\left(\Psi_{1} \mid: \widetilde{\phi}_{+}\left(\vec{p}-\vec{q}_{1}\right) \widetilde{\phi}_{+}\left(\vec{q}_{1}-\vec{q}_{2}\right) \ldots \widetilde{\phi}_{+}\left(\vec{q}_{n-1}\right): \Psi_{2}\right)\right|^{2},
\end{aligned}
$$

which, according to Lemma C.1.4 belong to $L^{1}\left(\mathbb{R}^{s}, d^{s} p\right)$ and their $L^{1}$-norms are uniformly bounded in $\Psi_{1}, \Psi_{2}$ from the above set. We introduce the function $\chi_{E}(\vec{p}) \in C_{0}^{\infty}\left(\mathbb{R}^{s}\right)$ which is equal to one on the set $\left\{\vec{p} \in \mathbb{R}^{s}|| \vec{p} \mid \leq E\right\}$ and obtain the following string of inequalities

$$
\begin{aligned}
& \quad \int d^{s} p|\vec{p}|^{\beta}\left|\left(\Psi_{1} \mid: \widetilde{\phi}_{+}^{n}:(\vec{p}) \Psi_{2}\right)\right|^{2} \\
& \leq(2 \pi)^{-s(n-1)} \int d^{s} p|\vec{p}|^{\beta} \mid \int d^{s} q_{1} \ldots d^{s} q_{n-1} \frac{\chi_{E}\left(\vec{p}-\vec{q}_{1}\right)}{\left|\vec{p}-\vec{q}_{1}\right|} \frac{\chi_{E}\left(\vec{q}_{1}-\vec{q}_{2}\right)}{\left|\vec{q}_{1}-\vec{q}_{2}\right|} \ldots \frac{\chi_{E}\left(\vec{q}_{n-1}\right)}{\left|\vec{q}_{n-1}\right|} \\
& \left.\quad \cdot\left|\vec{p}-\vec{q}_{1}\right|\left|\vec{q}_{1}-\vec{q}_{2}\right| \ldots\left|\vec{q}_{n-1}\right|\left(\Psi_{1} \mid: \widetilde{\phi}_{+}\left(\vec{p}-\vec{q}_{1}\right) \widetilde{\phi}_{+}\left(\vec{q}_{1}-\vec{q}_{2}\right) \ldots \widetilde{\phi}_{+}\left(\vec{q}_{n-1}\right): \Psi_{2}\right)\right|^{2} \\
& \leq(2 \pi)^{-s(n-1)} n^{\beta+1}|| F_{n} \|_{1} \\
& \sup _{\vec{p}_{1} \in \mathbb{R}^{s}} \int d^{s} q_{1} \ldots d^{s} q_{n-1} \frac{\left|\chi_{E}\left(\vec{p}_{1}-\vec{q}_{1}\right)\right|^{2}}{\left|\vec{p}_{1}-\vec{q}_{1}\right|^{2-\beta}} \frac{\left|\chi_{E}\left(\vec{q}_{1}-\vec{q}_{2}\right)\right|^{2}}{\left|\vec{q}_{1}-\vec{q}_{2}\right|^{2}} \ldots \frac{\left|\chi_{E}\left(\vec{q}_{n-1}\right)\right|^{2}}{\left|\vec{q}_{n-1}\right|^{2}} .
\end{aligned}
$$

Here in the first step we made use of the fact that the Fourier transform of a product is a convolution of the Fourier transforms of the factors. We also used the support properties 
of the wavefunctions corresponding to $\Psi_{1}, \Psi_{2}$. In the second step we applied the CauchySchwarz inequality and the bound $|\vec{p}|^{\beta} \leq n^{\beta}\left(\left|\vec{p}-\vec{q}_{1}\right|^{\beta}+\left|\vec{q}_{1}-\vec{q}_{2}\right|^{\beta} \cdots+\left|\vec{q}_{n-1}\right|^{\beta}\right)$ valid for any $\beta \geq 0$. To show that expression (C.1.24) is bounded in the cases considered in the lemma, we make use of the Young inequality [RS2] which implies that for $f_{i} \in L^{r_{i}}\left(\mathbb{R}^{s}, d^{s} p\right)$, $i \in\{1, \ldots, n\}$, there holds

$$
\left\|f_{1} * \cdots * f_{n}\right\|_{r} \leq\left\|f_{1}\right\|_{r_{1}} \ldots\left\|f_{n}\right\|_{r_{n}}
$$

if $1 \leq r, r_{i} \leq \infty$ are s.t. $r_{1}^{-1}+\cdots+r_{n}^{-1}=n-1+r^{-1}$. First, we assume that $\beta \geq 2$. Then, by choosing $r_{1}^{-1}=0, r_{2}^{-1}=\cdots=r_{n}^{-1}=1$, we conclude the proof of estimate (C.1.22). For $0 \leq \beta<2$, it follows from our assumptions that there exists $\varepsilon>0$ s.t. $(2-\beta)(1+\varepsilon)<$ $\min (s,(s-2)(n-1))$. We choose $r_{1}^{-1}=\frac{(2-\beta)(1+\varepsilon)}{s}, r_{2}^{-1}=\cdots=r_{n}^{-1}=1-\frac{r_{1}^{-1}}{(n-1)}$. One easily checks that these parameters satisfy the conditions specified above and, moreover, $(2-\beta) r_{1}<s, 2 r_{i}<s$ for $i \in\{2, \ldots, n\}$.

Proof of Proposition C.1.2 (b) and (c): Upper bounds from part (b) and part (c) follow from Lemma C.1.5 It remains to prove the lower bound in part (b) for $s=3$. Let $H_{n}$ be defined as in the proof of Proposition C.1.2 (a) above and let us consider the family of functionals from $\mathcal{T}_{E, 1}$ given by

$$
\varphi_{n}^{(2)}(\cdot)=\left(H_{n}^{\otimes(n-2)} \mid \cdot H_{n}^{\otimes n}\right) .
$$

We fix $\varepsilon>0$ and compute

$$
\begin{aligned}
\int d^{s} p|\vec{p}|^{1-\varepsilon} & \left|\varphi_{n}^{(2)}\left(: \widetilde{\phi}_{+}^{2}:(\vec{p})\right)\right|^{2} \\
= & \frac{(2 \pi)^{-s}}{4} \int d^{s} p|\vec{p}|^{1-\varepsilon}\left|\int d^{s} q \frac{1}{|\vec{p}-\vec{q}|^{\frac{1}{2}}|\vec{q}|^{\frac{1}{2}}}\left(H_{n}^{\otimes(n-2)} \mid a(\vec{p}-\vec{q}) a(\vec{q}) H_{n}^{\otimes n}\right)\right|^{2} \\
= & \frac{(2 \pi)^{-s}}{4} \frac{n^{3}(n-1)}{n^{s+1}} n^{\varepsilon} \int d^{s} p|\vec{p}|^{1-\varepsilon}\left|\int d^{s} q \frac{h(\vec{p}-\vec{q})}{|\vec{p}-\vec{q}|^{\frac{1}{2}}} \frac{h(\vec{q})}{|\vec{q}|^{\frac{1}{2}}}\right|^{2}
\end{aligned}
$$

By taking the limit $n \rightarrow \infty$, we verify that $\operatorname{ord}\left(: \phi_{+}^{2}:\right)=1$ for $s=3$. In the case of $: \phi_{+}^{2}: \in \Phi_{\mathrm{FH}}^{(\mathrm{e})}$ the lower bound is established analogously, restricting attention to even $n$. Similarly, the upper bound established in Lemma C.1.5 still holds, since the supremum in relation (C.1.22) extends now over a smaller set of functionals.

\section{C.2 Proof of Theorem C.1.1 (I): Functionals $\left\{\tau_{i}\right\}_{1}^{3}$}

In Section B.5 we introduced the decomposition of the map $\Theta_{E}: \mathfrak{A}(\mathcal{O}(r)) \rightarrow B(\mathcal{H})$, given by $\Theta_{E}(A)=P_{E} A P_{E}$, into three components

$$
\Theta_{E}=\hat{\Theta}_{E, 1}+\stackrel{\vee}{\Theta}_{E, 1}+\Theta_{E, 2},
$$

which are determined by the sets $\mathcal{S}$ and $\mathcal{M}$. With the statement of Theorem C.1.1 in mind, we choose $K^{+}=3, K^{-}=0$ in the definition of the set $\mathcal{S}$ and define the set $\mathcal{M}$ as consisting of four 2-multiindices

$$
\mathcal{M}=\left\{\bar{\alpha}_{0}, \ldots, \bar{\alpha}_{3}\right\}
$$


which are given by: $\alpha_{k}^{+}(j)=k \delta_{j, 1}, \alpha_{k}^{-}(j)=0$ for $k \in\{0,1,2,3\}, j \in \mathbb{N}$. Then, making use of definition (B.5.15) of the map $\hat{\Theta}_{E, 1}$ and recalling that our numbering of the $s$-indices $\left\{\kappa_{j}\right\}_{1}^{\infty}$, introduced after formula $(\underline{B .4 .3})$, was chosen so that $\kappa_{1}=0$, we obtain

$$
\hat{\Theta}_{E, 1}(A)=P_{E}\left(\omega_{0}(A) I+\hat{\tau}_{\bar{\alpha}_{1}}^{(r)}(A) \phi+\hat{\tau}_{\bar{\alpha}_{2}}^{(r)}(A): \phi^{2}:+\hat{\tau}_{\bar{\alpha}_{3}}^{(r)}(A): \phi^{3}:\right) P_{E},
$$

for any $A \in \mathfrak{A}(\mathcal{O}(r))$. Now we are in position to construct the functionals $\tau_{k}, k \in\{1,2,3\}$, appearing in Theorem C.1.1, and verify that they have the required properties.

Proposition C.2.1. In massless scalar free field theory for $s \geq 3$ there exist linear functionals $\tau_{k}, k \in\{1,2,3\}$ on $\hat{\mathfrak{A}}$ which satisfy

$$
\begin{aligned}
& \left.\tau_{k}\right|_{\mathfrak{A}(\mathcal{O}(r))}=\hat{\tau}_{\bar{\alpha}_{k}}^{(r)}, \\
& \tau_{k}(A(\vec{x}))=\tau_{k}(A)
\end{aligned}
$$

for any $r>0, A \in \hat{\mathfrak{A}}$ and $\vec{x} \in \mathbb{R}^{s}$. Moreover, $\omega_{0}, \tau_{1}, \tau_{2}, \tau_{3}$ form a linearly independent family. In addition there holds:

$$
\begin{aligned}
& \hat{\mathfrak{A}}^{(e)} \subset \operatorname{ker} \tau_{1} \cap \operatorname{ker} \tau_{3} \text { and }\left.\tau_{2}\right|_{\hat{\mathfrak{A}}^{(e)}} \neq 0, \\
& \hat{\mathfrak{A}}^{(d)} \subset \operatorname{ker} \tau_{1} \cap \operatorname{ker} \tau_{2} \cap \operatorname{ker} \tau_{3} .
\end{aligned}
$$

Proof. Let $\bar{\alpha}_{k}, k \in\{1,2,3\}$ be the multiindices introduced in (C.2.2) above. By definition (B.5.14), there holds

$$
\hat{\tau}_{\bar{\alpha}_{k}}^{(r)}=\frac{(i \sqrt{2})^{|k|}}{k !} \bar{\sigma}_{\bar{\alpha}_{k}}^{(r)}
$$

where $\bar{\sigma}_{\bar{\alpha}_{k}}^{(r)}$ are the functionals introduced in Lemma B.3.1, corresponding to the families of vectors $\left\{b_{\kappa_{j}, r}^{ \pm}\right\}_{j \in \mathbb{N}}$. Thus for any $f \in{\stackrel{\mathcal{L}}{r_{0}}}$ and $r \geq r_{0}$ we obtain

$$
\bar{\sigma}_{\bar{\alpha}_{k}}^{(r)}(W(f))=e^{-\frac{1}{2}\|f\|_{2}^{2}}\left\langle b_{0, r}^{+} \mid f^{+}\right\rangle^{k},
$$

where we made use of our convention that $\kappa_{1}=0$. We recall that $\tilde{f}^{ \pm}=\omega^{\mp \frac{1}{2}} \tilde{F}^{ \pm}$, where $F^{ \pm} \in D\left(\mathcal{O}_{r_{0}}\right)_{\mathbb{R}}$. Moreover, by definition (B.4.1),

$$
\tilde{b}_{0, r}^{ \pm}(\vec{p})=\frac{1}{(2 \pi)^{\frac{s}{2}}} \omega(\vec{p})^{ \pm \frac{1}{2}} \widetilde{\chi\left(\mathcal{O}_{r}\right)}(\vec{p})
$$

where $\chi\left(\mathcal{O}_{r}\right)=1$ on $\mathcal{O}_{r}$. We note that for $r \geq r_{0}$ and any $\vec{y} \in \mathbb{R}^{s}$ s.t. $\mathcal{O}_{r_{0}}+\vec{y} \subset \mathcal{O}_{r}$ there holds

$$
\begin{aligned}
\left\langle b_{0, r}^{+} \mid U(\vec{y}) f^{+}\right\rangle=\frac{1}{(2 \pi)^{\frac{s}{2}}}\left\langle\chi\left(\mathcal{O}_{r}\right) \mid U(\vec{y}) F^{+}\right\rangle & =\frac{1}{(2 \pi)^{\frac{s}{2}}} \int d^{s} x \chi\left(\mathcal{O}_{r}\right)(\vec{x}) F^{+}(\vec{x}-\vec{y}) \\
& =\frac{1}{(2 \pi)^{\frac{s}{2}}} \int d^{s} x F^{+}(\vec{x}),
\end{aligned}
$$

i.e the functions $r \rightarrow\left\langle b_{0, r}^{+} \mid U(\vec{y}) f^{+}\right\rangle$are constant for $r \geq r_{0}$ and independent of $\vec{y}$ within the above restrictions. Thus for any $r, r^{\prime} \geq r_{0}, A \in \mathfrak{A}\left(\mathcal{O}\left(r_{0}\right)\right)$ (i.e. $A$ is a finite linear combination of Weyl operators) and $\vec{y}$ as specified above, we obtain

$$
\begin{aligned}
& \hat{\tau}_{\bar{\alpha}_{k}}^{(r)}(A)-\hat{\tau}_{\bar{\alpha}}^{\left(r^{\prime}\right)}(A)=0, \\
& \hat{\tau}_{\bar{\alpha}_{k}}^{(r)}(A)-\hat{\tau}_{\bar{\alpha}}^{(r)}(A(\vec{y}))=0 .
\end{aligned}
$$


Since the functionals above are normal, the identities extend to any $A$ from $\mathfrak{A}\left(\mathcal{O}\left(r_{0}\right)\right)$. In view of the above relation and the fact that any $A \in \hat{\mathfrak{A}}$ belongs to $\mathfrak{A}\left(\mathcal{O}\left(r_{0}\right)\right)$ for sufficiently large $r_{0}$, the following formulas

$$
\tau_{k}(A)=\lim _{r \rightarrow \infty} \hat{\tau}_{\bar{\alpha}_{k}}^{(r)}(A), \quad A \in \hat{\mathfrak{A}}
$$

define linear functionals on $\hat{\mathfrak{A}}$ which satisfy conditions (C.2.4) and (C.2.5). By this former condition, relation (C.2.8) and formula (C.2.11), we obtain

$$
\tau_{k}(W(f))=\hat{\tau}_{\bar{\alpha}_{k}}^{\left(r_{0}\right)}(W(f))=\frac{(i \sqrt{2})^{|k|}}{k !} e^{-\frac{1}{2}\|f\|_{2}^{2}}\left(\frac{1}{(2 \pi)^{\frac{s}{2}}} \int d^{s} x F^{+}(\vec{x})\right)^{k},
$$

for $f, F^{+}$defined as above. The last integral vanishes if $F^{+}(\vec{x})=\sum_{j=1}^{s} \partial_{x_{j}} F_{j}^{+}(\vec{x})$ for some $F_{j}^{+} \in D\left(\mathcal{O}_{r_{0}}\right)_{\mathbb{R}}$. From this fact and the strong continuity of $\hat{\tau}_{\bar{\alpha}_{k}}\left(r_{0}\right)$ there follows statement (C.2.7). Next, we note that, by formula (C.2.15),

$$
\tau_{k}(W(f)+W(-f))=\hat{\tau}_{\bar{\alpha}_{k}}^{\left(r_{0}\right)}(W(f)+W(-f))=\left(1+(-1)^{k}\right) \hat{\tau}_{\bar{\alpha}_{k}}^{\left(r_{0}\right)}(W(f)) .
$$

Thus for $k \in\{1,3\}$ the functionals $\hat{\tau}_{\bar{\alpha}_{k}}^{\left(r_{0}\right)}$ are zero on $\mathfrak{A}^{(\mathrm{e})}\left(\mathcal{O}\left(r_{0}\right)\right)$, since they vanish on a strongly dense subspace of this algebra. This implies that $\hat{\mathfrak{A}}^{(\mathrm{e})}$ belongs to $\operatorname{ker} \tau_{1} \cap \operatorname{ker} \tau_{3}$. Choosing $f$ so that $\int d^{s} x F^{+}(\vec{x}) \neq 0$, we obtain from formulas (C.2.15), (C.2.16) that $\tau_{2}((W(f)+W(-f)) \neq 0$, what concludes the proof of statement (C.2.6).

We still have to show that the functionals $\omega_{0}, \tau_{1}, \tau_{2}, \tau_{3}$ are linearly independent. Suppose that

$$
c_{0} \omega_{0}+c_{1} \tau_{1}+c_{2} \tau_{2}+c_{3} \tau_{3}=0
$$

for some complex numbers $c_{0}, \ldots, c_{3}$. Evaluating this expression on the unity operator we obtain from relation (C.2.9) that $c_{0}=0$. Since there exist $A \in \hat{\mathfrak{A}}^{(\mathrm{e})}$ s.t. $\tau_{2}(A) \neq 0$, we get $c_{2}=0$. It remains to find $B \in \hat{\mathfrak{A}}$ s.t. $\tau_{1}(B)=0$ and $\tau_{3}(B) \neq 0$. Given $f, F^{+}$as introduced above, with the additional condition that $\int d^{s} x F^{+}(\vec{x}) \neq 0$, we pick a function $h \in S(\mathbb{R})$ s.t.

$$
\begin{aligned}
& \int e^{-\frac{1}{2} u^{2}\|f\|_{2}^{2}} u h(u) d u=0, \\
& \int e^{-\frac{1}{2} u^{2}\|f\|_{2}^{2}} u^{3} h(u) d u=1 .
\end{aligned}
$$

(The existence of such function can be established with the help of the Gram-Schmidt procedure as in the proof of Lemma D.3.1). The weak integral

$$
B:=\int W(u f) h(u) d u
$$

defines an element of $\mathfrak{A}\left(\mathcal{O}\left(r_{0}\right)\right)$ by the von Neumann bicommutant theorem. With the help of relations (C.2.8), (C.2.9) and (C.2.11) as well as properties (C.2.18), (C.2.19) of the function $h$ we obtain that $\tau_{1}(B)=0$ and $\tau_{3}(B) \neq 0$. Since $\tau_{1}$ is non-zero, this concludes the proof of linear independence of the functionals. 
The functionals $\tau_{k}, k \in\{1,2,3\}$, constructed in the above proposition, determine the map $R^{(2)}$ introduced in the statement of Theorem C.1.1 For any $A \in \mathfrak{A}(\mathcal{O}(r))$ we obtain

$$
R^{(2)}(A)=A-\omega_{0}(A) I-\tau_{1}(A) \phi_{+}-\tau_{2}(A): \phi_{+}^{2}:-\tau_{3}(A): \phi_{+}^{3}:
$$

in the sense of quadratic forms on the domain $D_{B} \times D_{B}$ of vectors of bounded energy. In view of property (C.2.4), formula (C.2.3) and decomposition (C.2.1) there holds

$$
P_{E} R^{(2)}(A) P_{E}=\stackrel{\vee}{\Theta}_{E, 1}(A)+\Theta_{E, 2}(A), \quad A \in \mathfrak{A}(\mathcal{O}(r)) .
$$

Thus to conclude the proof of Theorem C.1.1, we have to verify that the ranges of the maps $\stackrel{\vee}{\Theta}_{E, 1}, \Theta_{E, 2}$ are square-integrable. This is the subject of the next section.

\section{C.3 Proof of Theorem C.1.1 (II): Square-Integrability of $R^{(2)}$}

In this section we complete the proof of Theorem C.1.1. After introducing the necessary technical background in Subsection C.3.1, we prove the square-integrability of the ranges of the maps $\stackrel{\vee}{\Theta}_{E, 1}$ and $\Theta_{E, 2}$ in Subsections C.3.2 and C.3.3, respectively.

\section{C.3.1 Key Lemma}

The main goal of this subsection is to prove Lemma C.3.1 below, which is inspired by Lemma 2.2 of Bu90. To state and prove this result, it is convenient to proceed to the full (non-symmetrized) Fock space $\widehat{\mathcal{H}}$ on which there act the (prototype) creation operators $b^{*}(\Psi), \Psi \in L^{2}\left(\mathbb{R}^{s}, d^{s} p\right)$, given by

$$
b^{*}(\Psi) \Phi=\Psi \otimes \Phi, \quad \Phi \in \widehat{\mathcal{H}}
$$

and their adjoints $b(\Psi)$. Upon restriction to the symmetric Fock space $\mathcal{H}$, the formula $a(\Psi)=\sqrt{\widehat{N}} b(\Psi)$, where $\widehat{N}$ is the number operator, gives the standard annihilation operator introduced in Subsection B.2. With these definitions at hand we proceed to the main technical result of this appendix.

Lemma C.3.1. Let $E \geq 0$ and $h$ be a Borel function on $\mathbb{R}^{s}$ which is bounded on $\{\vec{p} \in$ $\left.\mathbb{R}^{s} \mid \omega(\vec{p}) \leq E\right\}$. We denote the operator of multiplication by $h$ on $L^{2}\left(\mathbb{R}^{s}, d^{s} p\right)$ by the same symbol. Let $\left\{g_{1, i}\right\}_{1}^{\infty},\left\{g_{2, i}\right\}_{1}^{\infty}$ be two families of functions from $L^{2}\left(\mathbb{R}^{s}, d^{s} p\right)$ which belong to the domain of $h \omega^{\frac{1}{2}}$ and let $\mu, \nu$ be multiindices. Then

$$
\begin{array}{r}
\sup _{\varphi \in \mathcal{T}_{E, 1}^{+}} \int_{K} d^{s} x \varphi\left(a^{*}\left(h \omega^{\frac{1}{2}} g_{1, \vec{x}}\right)^{\mu} a\left(h \omega^{\frac{1}{2}} g_{1, \vec{x}}\right)^{\mu}\right) \varphi\left(a^{*}\left(h \omega^{\frac{1}{2}} g_{2, \vec{x}}\right)^{\nu} a\left(h \omega^{\frac{1}{2}} g_{2, \vec{x}}\right)^{\nu}\right) \\
\leq\left(\sup _{\omega(\vec{p}) \leq E}|h(\vec{p})|^{2} E\right)^{|\mu|+|\nu|} \int_{\Delta K} d^{s} y\left|\left(\Omega \mid b\left(g_{1}\right)^{\mu} b^{*}\left(g_{1, \vec{y}}\right)^{\mu} \Omega\right)\right| \\
\left|\left(\Omega \mid b\left(g_{2}\right)^{\nu} b^{*}\left(g_{2, \vec{y}}\right)^{\nu} \Omega\right)\right|
\end{array}
$$

where $K$ is any compact subset of $\mathbb{R}^{s}, \Delta K=\{\vec{x}-\vec{y} \mid \vec{x}, \vec{y} \in K\}, g_{k, i, \vec{x}}=U_{1}(\vec{x}) g_{k, i}$, $k \in\{1,2\}, i \in \mathbb{N}$. 
Proof. Let $Q=\int_{K} d^{s} x b^{*}\left(g_{1, \vec{x}}\right)^{\mu} b\left(g_{1, \vec{x}}\right)^{\mu} \otimes b^{*}\left(g_{2, \vec{x}}\right)^{\nu} b\left(g_{2, \vec{x}}\right)^{\nu}$ act on $\widehat{\mathcal{H}} \otimes \widehat{\mathcal{H}}$ and $\Psi_{1}, \Psi_{2} \in \widehat{\mathcal{H}}$ be $|\mu|-$, resp. $|\nu|-$, particle vectors. For $|\mu|=0$ (resp. $|\nu|=0$ ) we choose $\Psi_{1}$ (resp. $\Psi_{2}$ ) to be a multiple of $\Omega$. Then $\varphi(\cdot)=\left(\Psi_{1} \mid \cdot \Psi_{1}\right)\left(\Psi_{2} \mid \cdot \Psi_{2}\right)$ is a positive functional on $B(\widehat{\mathcal{H}}) \otimes B(\widehat{\mathcal{H}})$ and we obtain

$$
\begin{array}{r}
\varphi(Q)^{2} \leq \varphi(I) \varphi(Q Q)=\varphi(I) \int_{K} d^{s} x \int_{K} d^{s} y\left(\Psi_{1} \mid b^{*}\left(g_{1, \vec{x}}\right)^{\mu} b\left(g_{1, \vec{x}}\right)^{\mu} b^{*}\left(g_{1, \vec{y}}\right)^{\mu} b\left(g_{1, \vec{y}}\right)^{\mu} \Psi_{1}\right) \\
\left(\Psi_{2} \mid b^{*}\left(g_{2, \vec{x}}\right)^{\nu} b\left(g_{2, \vec{x}}\right)^{\nu} b^{*}\left(g_{2, \vec{y}}\right)^{\nu} b\left(g_{2, \vec{y}}\right)^{\nu} \Psi_{2}\right) \\
\leq \varphi(I) \int_{K} d^{s} x \int_{K} d^{s} y\left|\left(\Psi_{1} \mid b^{*}\left(g_{1, \vec{x}}\right)^{\nu} \Omega\right)\left(\Omega \mid b\left(g_{1, \vec{x}}\right)^{\nu} b^{*}\left(g_{1, \vec{y}}\right)^{\nu} \Omega\right)\left(\Omega \mid b\left(g_{1, \vec{y}}\right)^{\nu} \Psi_{1}\right)\right| \\
\left|\left(\Psi_{2} \mid b^{*}\left(g_{2, \vec{x}}\right)^{\nu} \Omega\right)\left(\Omega \mid b\left(g_{2, \vec{x}}\right)^{\nu} b^{*}\left(g_{2, \vec{y}}\right)^{\nu} \Omega\right)\left(\Omega \mid b\left(g_{2, \vec{y}}\right)^{\nu} \Psi_{2}\right)\right| \\
\leq \varphi(I) \varphi(Q) \int_{\Delta K} d^{s} y\left|\left(\Omega \mid b\left(g_{1}\right)^{\mu} b^{*}\left(g_{1, \vec{y}}\right)^{\mu} \Omega\right)\right|\left|\left(\Omega \mid b\left(g_{2}\right)^{\nu} b^{*}\left(g_{2, \vec{y}}\right)^{\nu} \Omega\right)\right|,
\end{array}
$$

where in the last step we made use of the inequality $|f(\vec{x}) f(\vec{y})| \leq \frac{1}{2}\left(|f(\vec{x})|^{2}+|f(\vec{y})|^{2}\right)$. Consequently

$$
\begin{aligned}
& \int_{K} d^{s} x\left(\Psi_{1} \mid b^{*}\left(g_{1, \vec{x}}\right)^{\mu} b\left(g_{1, \vec{x}}\right)^{\mu} \Psi_{1}\right)\left(\Psi_{2} \mid b^{*}\left(g_{2, \vec{x}}\right)^{\nu} b\left(g_{2, \vec{x}}\right)^{\nu} \Psi_{2}\right) \\
& \quad \leq\left\|\Psi_{1}\right\|^{2}\left\|\Psi_{2}\right\|^{2} \int_{\Delta K} d^{s} y\left|\left(\Omega \mid b\left(g_{1}\right)^{\mu} b^{*}\left(g_{1, \vec{y}}\right)^{\mu} \Omega\right)\right|\left|\left(\Omega \mid b\left(g_{2}\right)^{\nu} b^{*}\left(g_{2, \vec{y}}\right)^{\nu} \Omega\right)\right|
\end{aligned}
$$

We pick $n_{1}, n_{2} \in \mathbb{N}$ s.t. $n_{1} \geq|\mu|, n_{2} \geq|\nu|$. Given $n_{1^{-}}$and $n_{2}$-particle vectors $\Psi_{n_{1}}, \Psi_{n_{2}} \in$ $\left(P_{E} \mathcal{H}\right)_{1}$, whose wavefunctions are Schwartz-class, we define $|\mu|-$, resp. $|\nu|$-particle vectors, where the remaining arguments are treated as parameters

$$
\begin{aligned}
& \Psi_{1}\left(\vec{p}_{1}, \ldots, \vec{p}_{|\mu|}\right)_{\vec{p}_{|\mu|+1}, \ldots, \vec{p}_{n_{1}}}:=\bar{h}\left(\vec{p}_{1}\right) \omega\left(\vec{p}_{1}\right)^{\frac{1}{2}} \ldots \bar{h}\left(\vec{p}_{|\mu|}\right) \omega\left(\vec{p}_{|\mu|}\right)^{\frac{1}{2}} \Psi_{n_{1}}\left(\vec{p}_{1}, \ldots, \vec{p}_{n_{1}}\right), \\
& \Psi_{2}\left(\vec{q}_{1}, \ldots, \vec{q}_{|\nu|}\right)_{\vec{q}_{|\nu|+1}, \ldots, \vec{q}_{n_{2}}}:=\bar{h}\left(\vec{q}_{1}\right) \omega\left(\vec{q}_{1}\right)^{\frac{1}{2}} \ldots \bar{h}\left(\vec{q}_{|\nu|}\right) \omega\left(\vec{q}_{|\nu|}\right)^{\frac{1}{2}} \Psi_{n_{2}}\left(\vec{q}_{1}, \ldots, \vec{q}_{n_{2}}\right) .
\end{aligned}
$$

With these definitions we compute

$$
\begin{array}{r}
\int_{K} d^{s} x\left(\Psi_{n_{1}} \mid a^{*}\left(h \omega^{\frac{1}{2}} g_{1, \vec{x}}\right)^{\mu} a\left(h \omega^{\frac{1}{2}} g_{1, \vec{x}}\right)^{\mu} \Psi_{n_{1}}\right)\left(\Psi_{n_{2}} \mid a^{*}\left(h \omega^{\frac{1}{2}} g_{2, \vec{x}}\right)^{\nu} a\left(h \omega^{\frac{1}{2}} g_{2, \vec{x}}\right)^{\nu} \Psi_{n_{2}}\right) \\
=\frac{n_{1} !}{\left(n_{1}-|\mu|\right) !} \frac{n_{2} !}{\left(n_{2}-|\nu|\right) !} \int d^{s} p_{|\mu|+1} \ldots d^{s} p_{n_{1}} \int d^{s} q_{|\nu|+1} \ldots d^{s} q_{n_{2}} \\
\int_{K} d^{s} x\left(\Psi_{1, \vec{p}_{|\mu|+1}, \ldots, \vec{p}_{n_{1}} \mid} \mid b^{*}\left(g_{1, \vec{x}}\right)^{\mu} b\left(g_{1, \vec{x}}\right)^{\mu} \Psi_{1, \vec{p}_{|\mu|+1}, \ldots, \vec{p}_{n_{1}}}\right) \\
\left(\Psi_{2, \vec{q}_{|\nu|+1}, \ldots, \vec{q}_{n_{2}}} \mid b^{*}\left(g_{2, \vec{x}}\right)^{\nu} b\left(g_{2, \vec{x}}\right)^{\nu} \Psi_{2, \vec{q}_{|\nu|+1}, \ldots, \vec{q}_{n_{2}}}\right) \\
\leq\left(\sup _{|\vec{p}| \leq E}|h(\vec{p})|^{2} E\right)^{|\mu|+|\nu|} \int_{\Delta K} d^{s} y\left|\left(\Omega \mid b\left(g_{1}\right)^{\mu} b^{*}\left(g_{1, \vec{y}}\right)^{\mu} \Omega\right)\right| \\
\left|\left(\Omega \mid b\left(g_{2}\right)^{\nu} b^{*}\left(g_{2, \vec{y}}\right)^{\nu} \Omega\right)\right|,
\end{array}
$$

where in the last step we applied formula (C.3.4) and made use of the following computa- 
tion to control the $n_{1}$ and $n_{2}$ dependence

$$
\begin{aligned}
& \frac{n_{1} !}{\left(n_{1}-|\mu|\right) !} \int d^{s} p_{1} \ldots d^{s} p_{n_{1}} \omega\left(\vec{p}_{1}\right) \ldots \omega\left(\vec{p}_{|\mu|}\right)\left|\Psi_{n_{1}}\left(\vec{p}_{1}, \ldots, \vec{p}_{n_{1}}\right)\right|^{2} \\
& \quad=|\mu| ! \int d^{s} p_{1} \ldots d^{s} p_{n_{1}} \sum_{i_{1}<\ldots<i_{|\mu|}} \omega\left(\vec{p}_{i_{1}}\right) \ldots \omega\left(\vec{p}_{i_{|\mu|}}\right)\left|\Psi_{n_{1}}\left(\vec{p}_{1}, \ldots, \vec{p}_{n_{1}}\right)\right|^{2} \\
& \quad \leq \int d^{s} p_{1} \ldots d^{s} p_{n_{1}}\left(\omega\left(\vec{p}_{1}\right)+\cdots+\omega\left(\vec{p}_{n_{1}}\right)\right)^{|\mu|}\left|\Psi_{n_{1}}\left(\vec{p}_{1}, \ldots, \vec{p}_{n_{1}}\right)\right|^{2} \leq E^{|\mu|} .
\end{aligned}
$$

Here in the first step we used the symmetry of the wavefunction and in the second step we applied the multinomial formula. By an approximation argument, the bound (C.3.7) holds also without the restriction that $\Psi_{n_{1}}, \Psi_{n_{2}}$ have Schwartz-class wavefunctions.

To conclude the argument, we need several simple, geometrical observations: We note that any $\varphi \in \mathcal{T}_{E, 1}^{+}$has the form $\varphi(\cdot)=\sum_{k=1}^{\infty} p_{k}\left(\Psi_{k} \mid \cdot \Psi_{k}\right)$, where $p_{k} \geq 0, \sum_{k=1}^{\infty} p_{k} \leq 1$, and $\Psi_{k} \in\left(P_{E} \mathcal{H}\right)_{1}$. Let $C_{1}, C_{2}$ be a pair of bounded, positive operators. (The operators $P_{E} a^{*}\left(h \omega^{\frac{1}{2}} g_{1}\right)^{\mu} a\left(h \omega^{\frac{1}{2}} g_{1}\right)^{\mu} P_{E}, P_{E} a^{*}\left(h \omega^{\frac{1}{2}} g_{2}\right)^{\nu} a\left(h \omega^{\frac{1}{2}} g_{2}\right)^{\nu} P_{E}$, appearing on the l.h.s. of relation (C.3.7), are bounded due to the energy bounds (B.5.22) and the fact that $\left.\left\|Q_{E} h\right\|<\infty\right)$. There holds the simple estimate

$$
\begin{aligned}
\sup _{\varphi \in \mathcal{T}_{E, 1}^{+}} \int_{K} d^{s} x \varphi\left(C_{1}(\vec{x})\right) \varphi\left(C_{2}(\vec{x})\right) & \\
\leq & \sup _{\Psi_{1}, \Psi_{2} \in\left(P_{E} \mathcal{H}\right)_{1}} \int_{K} d^{s} x\left(\Psi_{1} \mid C_{1}(\vec{x}) \Psi_{1}\right)\left(\Psi_{2} \mid C_{2}(\vec{x}) \Psi_{2}\right) .
\end{aligned}
$$

Finally, we can decompose any $\Psi \in\left(P_{E} \mathcal{H}\right)_{1}$ as $\Psi=c_{0} \Omega+\sum_{n=1}^{\infty} c_{n} \Psi_{n}$, where $\Psi_{n}$ are normalized $n$-particle wavefunctions and $\left|c_{0}\right|^{2}+\sum_{n=1}^{\infty}\left|c_{n}\right|^{2} \leq 1$. Consequently, for $C_{1}$ and $\mathrm{C}_{2}$ as above, conserving particle number, we obtain

$$
\begin{aligned}
\int_{K} d^{s} x\left(\Psi_{1} \mid C_{1}(\vec{x})\right. & \left.\Psi_{1}\right)\left(\Psi_{2} \mid C_{2}(\vec{x}) \Psi_{2}\right) \\
& \leq \sup _{n_{1}, n_{2}} \int_{K} d^{s} x\left(\Psi_{1, n_{1}} \mid C_{1}(\vec{x}) \Psi_{1, n_{1}}\right)\left(\Psi_{2, n_{2}} \mid C_{2}(\vec{x}) \Psi_{2, n_{2}}\right) .
\end{aligned}
$$

Now the bound in the statement of the lemma follows from estimate (C.3.7).

In the next two subsections we will use the above lemma to prove the square-integrability of the individual terms appearing in the expansions of the maps $\stackrel{\vee}{\Theta}_{E, 1}$ and $\Theta_{E, 2}$. Then the square-integrability of the ranges of these maps follows from the special case of the Minkowski inequality [Hal] which we state below.

Lemma C.3.2. For any family of $f_{n} \in L^{2}\left(\mathbb{R}^{s}, d^{s} p\right), n \in \mathbb{N}$, whose norms $\left\|f_{n}\right\|_{2}$ form a summable sequence, there holds the bound

$$
\left(\int d^{s} p\left|\sum_{n=1}^{\infty} f_{n}(\vec{p})\right|^{2}\right)^{\frac{1}{2}} \leq \sum_{n=1}^{\infty}\left(\int d^{s} p\left|f_{n}(\vec{p})\right|^{2}\right)^{\frac{1}{2}} .
$$

After this technical preparation we can proceed with the proof of Theorem C.1.1. 


\section{C.3.2 Square-Integrability of $\stackrel{\vee}{\Theta}_{E, 1}$}

In this subsection we apply Lemma C.3.1 to prove the square-integrability of the range of the map $\stackrel{\vee}{\Theta}_{E, 1}$. In order to control the expressions appearing on the r.h.s. of estimate (C.3.2), we need the following result:

Lemma C.3.3. Let $\lambda>\frac{s}{2}$ and suppose that $\tilde{h} \in L^{2}\left(\mathbb{R}^{s}, d^{s} p\right)_{\lambda}$. Then the function $\mathbb{R}^{s} \ni$ $\vec{x} \rightarrow\langle\tilde{h} \mid U(\vec{x}) \tilde{h}\rangle$ is absolutely integrable and there holds

$$
\int d^{s} x|\langle\tilde{h} \mid U(\vec{x}) \tilde{h}\rangle| \leq c_{\lambda}\|\tilde{h}\|_{2, \lambda}^{2}
$$

where the constant $c_{\lambda}$ depends only on $\lambda$ and $s .($ Here $\langle\cdot \mid \cdot\rangle$ denotes the scalar product in $\left.L^{2}\left(\mathbb{R}^{s}, d^{s} p\right)\right)$.

Proof. We pick $\tilde{h} \in S\left(\mathbb{R}^{s}\right)$, a compact subset $K \subset \mathbb{R}^{s}$ and estimate

$$
\begin{aligned}
\int_{K} d^{s} x|\langle\tilde{h} \mid U(\vec{x}) \tilde{h}\rangle| & =\int_{K} d^{s} x\left|\int d^{s} p e^{-i \vec{p} \vec{x}} \bar{h}(\vec{p}) \tilde{h}(\vec{p})\right| \\
& \leq \int d^{s} x\left|\int d^{s} y \bar{h}(\vec{y}) h(\vec{y}+\vec{x})\right| \leq\left(\int d^{s} y|h(\vec{y})|\right)^{2} \\
& \leq \int d^{s} x \frac{1}{\left(1+|\vec{x}|^{2}\right)^{\lambda}} \int d^{s} y\left(1+|\vec{y}|^{2}\right)^{\lambda}|h(\vec{y})|^{2} \\
& =c_{\lambda}\|\tilde{h}\|_{2, \lambda}^{2},
\end{aligned}
$$

where in the fourth step we made use of the Cauchy-Schwarz inequality. Since $S\left(\mathbb{R}^{s}\right)$ is dense in $L^{2}\left(\mathbb{R}^{s}, d^{s} p\right)_{\lambda}$, this estimate extends to any $\tilde{h} \in L^{2}\left(\mathbb{R}^{s}, d^{s} p\right)_{\lambda}$. Finally, as the constant $c_{\lambda}$ is independent of $K$, we can take the limit $K \nearrow \mathbb{R}^{s}$.

With our choice of the sets $\mathcal{S}$ and $\mathcal{M}$, (see definition $(\mathbf{C . 2 . 2})$ ), the map $\stackrel{\vee}{\Theta}_{E, 1}$, given by formula (B.5.20), has the following form

$$
\stackrel{\vee}{\Theta}_{E, 1}(A)=\sum_{\substack{\mu^{+}, \nu^{+} \\ \exists j>1}} \stackrel{\vee}{\tau}_{\mu^{+}, \nu^{+}}^{(r)}(A) \stackrel{\vee}{\mu^{+}(j)+\nu^{+}(j) \neq 0} \mu_{\mu^{+}, \nu^{+}}, \quad A \in \mathfrak{A}(\mathcal{O}(r)),
$$

where we made use of the fact that $\mu^{-}=\nu^{-}=0$, since $K^{-}=0$. We recall from formula (B.5.19) that

$$
\stackrel{\vee}{S}_{\mu^{+}, \nu^{+}}=P_{E} a^{*}\left(h_{\kappa, E}^{+}\right)^{\mu^{+}} a\left(h_{\kappa, E}^{+}\right)^{\nu^{+}} P_{E}
$$

and that the numbering $\left\{\kappa_{j}\right\}_{1}^{\infty}$ of the $s$-indices is chosen so that $\kappa_{1}=0$. The following lemma establishes the square-integrability of these operators under the restrictions on the multiindices $\mu^{+}, \nu^{+}$given in the sum (C.3.14). We will exploit the useful fact that for any $C \in B(\mathcal{H})$ which satisfies $\|C\|_{E, 2}<\infty$ there holds

$$
\|C\|_{E, 2} \leq 4 \sup _{\varphi \in \mathcal{T}_{E, 1}^{+}}\left(\int d^{s} x|\varphi(C(\vec{x}))|^{2}\right)^{\frac{1}{2}} .
$$

This bound follows immediately from decomposition (1.6.2). 
Lemma C.3.4. Suppose that $\mu^{+}(j)+\nu^{+}(j) \neq 0$ for some $j>1$. Then in massless scalar free field theory for $s \geq 3$ there holds the bound

$$
\left\|\stackrel{\vee}{S}_{\mu^{+}, \nu^{+}}\right\|_{E, 2} \leq\left(c_{E}^{|\kappa|+1}\right)^{\mu^{+}+\nu^{+}}
$$

for some constant $c_{E}$ independent of $\kappa$.

Proof. We assume, without restriction, that there exist $j>1$ s.t. $\nu^{+}(j) \neq 0$. Thus we can choose multiindices $\nu_{a}^{+}, \nu_{b}^{+}$in such a way that $\nu^{+}=\nu_{a}^{+}+\nu_{b}^{+}$and $\left|\nu_{a}^{+}\right|=\nu_{a}^{+}(j)=1$. Making use of formula (C.3.15), we obtain for any compact subset $K \subset \mathbb{R}^{s}$

$$
\begin{aligned}
& \sup _{\varphi \in \mathcal{T}_{E, 1}^{+},} \int_{K} d^{s} x\left|\varphi\left(\stackrel{\vee}{S}_{\mu^{+}, \nu^{+}}(\vec{x})\right)\right|^{2} \\
& \leq \sup _{\varphi \in \mathcal{T}_{E, 1}^{+}} \int_{K} d^{s} x \varphi_{\vec{x}}\left(a^{*}\left(h_{\kappa, E}^{+}\right)^{\mu^{+}} a\left(h_{\kappa, E}^{+}\right)^{\mu^{+}}\right) \varphi_{\vec{x}}\left(a^{*}\left(h_{\kappa, E}^{+}\right)^{\nu^{+}} a\left(h_{\kappa, E}^{+}\right)^{\nu^{+}}\right) \\
& \leq E^{\left|\mu^{+}\right|+\left|\nu_{b}^{+}\right|}\left\|\omega^{-\frac{1}{2}} h_{\kappa, E}^{+}\right\|_{2}^{2\left(\left|\mu^{+}\right|+\left|\nu_{b}^{+}\right|\right)} \sup _{\varphi \in \mathcal{T}_{E, 1}^{+}} \int_{K} d^{s} x \varphi_{\vec{x}}\left(a^{*}\left(h_{\kappa_{j}, E}^{+}\right) a\left(h_{\kappa_{j}, E}^{+}\right)\right),
\end{aligned}
$$

where in the first step we applied the Cauchy-Schwarz inequality and in the second step we exploited the energy bounds (B.5.22), Lemma B.4.4 the fact that $a\left(h_{\kappa, E}\right)^{\nu^{+}} P_{E}=$ $a\left(h_{\kappa, E}\right)^{\nu_{b}^{+}} P_{E} a\left(h_{\kappa, E}\right)^{\nu_{a}^{+}} P_{E}$ and the properties of $\nu_{a}^{+}$.

We will apply Lemma C.3.1 to the integral on the r.h.s. of relation (C.3.18): Since $\kappa_{j} \neq 0$, we can write $\kappa_{j}=\hat{\kappa}_{j}+\stackrel{\vee}{\kappa}_{j}$ in such a way that $\left|\hat{\kappa}_{j}\right|=1$. Next, we set $h(\vec{p})=\frac{(i p)^{\hat{\kappa}_{j}}}{\omega(\vec{p})}$ for $\vec{p} \neq 0$ and $h(0)=0$. There holds $\|h\|_{\infty} \leq 1$ and $\tilde{h}_{\kappa_{j}, E}^{+}=h \omega^{\frac{1}{2}}\left(\omega^{\frac{1}{2}} \tilde{h}_{\aleph_{j}, E}^{+}\right)$. (We recall that $\tilde{h}_{\kappa, E}^{+}(\vec{p})=(-1)^{|\kappa|} \omega(\vec{p})^{-\frac{1}{2}}(i p)^{\kappa} \chi_{E}(\vec{p})$ by definition (B.4.2) $)$. Moreover, by Lemma B.4.4 there holds for any $\lambda \geq 0$

$$
\begin{aligned}
& \left\|\omega^{\frac{1}{2}} \tilde{h}_{\kappa, E}^{+}\right\|_{2, \lambda} \leq\left(c_{\lambda,-\frac{1}{2}, E}\right)^{|\kappa|+1}, \\
& \left\|\omega^{-\frac{1}{2}} \tilde{h}_{\kappa, E}^{+}\right\|_{2} \leq\left(c_{0, \frac{1}{2}, E}\right)^{|\kappa|+1},
\end{aligned}
$$

where the constants $c_{\lambda,-\frac{1}{2}, E}, c_{0, \frac{1}{2}, E}$ are independent of $\kappa$. We pick some $\lambda>\frac{s}{2}$ and obtain from Lemmas C.3.1 and C.3.3

$$
\begin{aligned}
\sup _{\varphi \in \mathcal{T}_{E, 1}^{+}} \int_{K} d^{s} x \varphi_{\vec{x}}\left(a^{*}\left(h_{\kappa_{j}, E}^{+}\right) a\left(h_{\kappa_{j}, E}^{+}\right)\right) & \leq E \int_{\Delta K} d^{s} x\left|\left\langle\omega^{\frac{1}{2}} \tilde{h}_{\kappa_{j}, E}^{+} \mid U(\vec{x}) \omega^{\frac{1}{2}} \tilde{h}_{\kappa_{j}, E}^{+}\right\rangle\right| \\
& \leq E c_{\lambda}\left\|\omega^{\frac{1}{2}} \tilde{h}_{\kappa_{\kappa_{j}, E}^{+}}^{+}\right\|_{2, \lambda}^{2} \\
& \leq\left(c_{E}\right)^{\left|\kappa_{j}\right|+1}=\left(\left(c_{E}\right)^{|\kappa|+1}\right)^{\nu_{a}^{+}}
\end{aligned}
$$

Here in the third step we used the bound (C.3.19), absorbed the constants involved into one constant $c_{E}$, independent of $\stackrel{\vee}{\kappa}_{j}$, and made use of the fact that $\left|\kappa_{j}\right|=\left|\aleph_{j}\right|+1$. Substituting the above bound to relation (C.3.18) and making use of estimates (C.3.20) and (C.3.16), we obtain the estimate in the statement of the lemma (after readjusting the constant $c_{E}$ ).

After this preparation we can prove the square-integrability of the range of the map $\stackrel{\vee}{\Theta}_{E, 1}$. 
Proposition C.3.5. In massless scalar free field theory for $s \geq 3$ the map $\stackrel{\vee}{\Theta}_{E, 1}$, given by relation (C.3.14), satisfies the bound

$$
\sup _{A \in \mathfrak{A}(\mathcal{O}(r))_{1}}\left\|\stackrel{\vee}{\Theta}_{E, 1}(A)\right\|_{E, 2}<\infty .
$$

Proof. First, we recall that due to the bound (B.5.21) and Lemma B.4.3 there holds

$$
\begin{aligned}
\left\|{\stackrel{\mathcal{\tau}}{\mu^{+}, \nu^{+}}}^{(r)}\right\| & \leq 2^{\left|\mu^{+}\right|+\left|\nu^{+}\right|} \sqrt{\left(\left|\mu^{+}\right|+\left|\nu^{+}\right|\right) !}\left\|b_{\kappa, r}\right\|_{2}^{\mu^{+}+\nu^{+}} \\
& \leq \sqrt{\left(\left|\mu^{+}\right|+\left|\nu^{+}\right|\right) !}\left(\frac{\left(c_{r}\right)^{|\kappa|+1}}{\kappa !}\right)^{\mu^{+}+\nu^{+}}
\end{aligned}
$$

for some constant $c_{r}$, independent of $\kappa$. Next, making use of the Minkowski inequality (C.3.11), we obtain for any $A \in \mathfrak{A}(\mathcal{O}(r))_{1}$

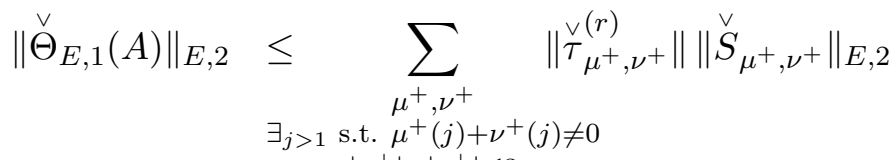

$$
\begin{aligned}
& \left|\mu^{+}\right|+\left|\nu^{+}\right| \leq 3 \\
& \leq \sum_{\substack{\mu^{+}, \nu^{+} \\
\left|\mu^{+}\right|+\left|\nu^{+}\right| \leq 3}} \sqrt{\left(\left|\mu^{+}\right|+\left|\nu^{+}\right|\right) !}\left(\frac{\left(c_{r} c_{E}\right)^{|\kappa|+1}}{\kappa !}\right)^{\mu^{+}+\nu^{+}} \\
& \leq \sqrt{3 !}\left(\sum_{\mu^{+},\left|\mu^{+}\right| \leq 3}\left(\frac{\left(c_{r} c_{E}\right)^{|\kappa|+1}}{\kappa !}\right)^{\mu^{+}}\right)^{2} \\
& \leq \sqrt{3 !}\left(\sum_{k=0}^{3}\left(\sum_{\kappa \in \mathbb{N}_{0}^{s}} \frac{\left(c_{r} c_{E}\right)^{|\kappa|+1}}{\kappa !}\right)^{k}\right)^{2}
\end{aligned}
$$

where in the second step we made use of the bounds (C.3.23), (C.3.17). In the last step we used the fact that the multinomial coefficients are larger or equal to one and we applied the multinomial formula (B.1.4). The last expression is clearly finite.

\section{C.3.3 Square-Integrability of $\Theta_{E, 2}$}

Now we proceed to the proof of the square-integrability of the range of the map $\Theta_{E, 2}$, defined by expansion (B.5.37). Again, we will first apply Lemma C.3.1 to the individual terms of this expansion and then use the Minkowski inequality (C.3.11) to conclude the proof. In order to control the terms appearing on the r.h.s. of estimate (C.3.2), we need two auxiliary lemmas proven below.

In Subsection B.4.1 we introduced, for any $\rho>0$, a function $\chi\left(\mathcal{O}_{\rho}\right) \in C_{0}^{\infty}\left(\mathbb{R}^{s}\right)$ s.t. $\chi\left(\mathcal{O}_{\rho}\right)(\vec{x})=1$ for $\vec{x} \in \mathcal{O}_{\rho}$. Here we demand in addition that $\chi\left(\mathcal{O}_{\rho}\right)(\vec{x})=0$ for $\vec{x} \notin \mathcal{O}_{\rho+\tilde{\varepsilon}}$, for some fixed $\tilde{\varepsilon}>0$, independent of $\rho$. We denote the operator of multiplication by $\chi\left(\mathcal{O}_{\rho}\right)$ in configuration space by the same symbol.

Lemma C.3.6. Suppose that $F \in S^{\prime}\left(\mathbb{R}^{s}\right)$ coincides with a bounded, measurable function in the region $\left\{\vec{y} \in \mathbb{R}^{s}|| \vec{y} \mid \geq \rho\right\}$ and its Fourier transform $\tilde{F}$ is a positive, measurable 
function s.t. $\tilde{F}^{1 / 2} \in L^{2}\left(\mathbb{R}^{s}, d^{s} p\right)+L^{\infty}\left(\mathbb{R}^{s}, d^{s} p\right)$. Then $\tilde{F}^{1 / 2} \chi\left(\mathcal{O}_{\rho}\right)$ is a bounded operator and there holds

$$
\left\|\chi\left(\mathcal{O}_{\rho}\right) \tilde{F} \chi_{\vec{x}}\left(\mathcal{O}_{\rho}\right)\right\| \leq c_{\rho, \tilde{\varepsilon}} \sup _{|\vec{z}| \leq 2 \rho+3 \tilde{\varepsilon}}|F(\vec{z}+\vec{x})| \text { for }|\vec{x}| \geq 3(\rho+\tilde{\varepsilon}),
$$

where $\chi_{\vec{x}}\left(\mathcal{O}_{\rho}\right)(\vec{y})=\chi\left(\mathcal{O}_{\rho}\right)(\vec{y}-\vec{x})$, the constant $c_{\rho, \tilde{\varepsilon}}$ is independent of $\vec{x}$ and we denote the operator of multiplication by $\tilde{F}$ in momentum space by the same symbol.

Proof. In order to prove the first statement, we make a decomposition $\tilde{F}^{1 / 2}=\tilde{F}_{2}^{1 / 2}+\tilde{F}_{\infty}^{1 / 2}$, where $\tilde{F}_{2}^{1 / 2} \in L^{2}\left(\mathbb{R}^{s}, d^{s} p\right), \tilde{F}_{\infty}^{1 / 2} \in L^{\infty}\left(\mathbb{R}^{s}, d^{s} p\right)$. Since $\tilde{F}_{\infty}^{1 / 2}$ is a bounded operator, it suffices to consider $\tilde{F}_{2}^{1 / 2} \chi\left(\mathcal{O}_{\rho}\right)$. We pick $f_{1}, f_{2} \in S\left(\mathbb{R}^{s}\right)$ and estimate

$$
\begin{array}{r}
\left|\left\langle f_{1} \mid \tilde{F}_{2}^{1 / 2} \chi\left(\mathcal{O}_{\rho}\right) f_{2}\right\rangle\right|=(2 \pi)^{-\frac{s}{2}}\left|\int d^{s} p d^{s} q \overline{\tilde{f}}_{1}(\vec{p}) \tilde{F}_{2}^{1 / 2}(\vec{p}) \widetilde{\chi\left(\mathcal{O}_{\rho}\right)}(\vec{p}-\vec{q}) \tilde{f}_{2}(\vec{q})\right| \\
\leq c\left\|\tilde{\tilde{f}}_{1} \tilde{F}_{2}^{1 / 2}\right\|_{1}\left\|\chi\left(\mathcal{O}_{\rho}\right)\right\|_{2}\left\|f_{2}\right\|_{2} \leq c\left\|f_{1}\right\|_{2}\left\|\tilde{F}_{2}^{1 / 2}\right\|_{2}\left\|\chi\left(\mathcal{O}_{\rho}\right)\right\|_{2}\left\|f_{2}\right\|_{2},
\end{array}
$$

where in the second step we made use of the Young inequality 1 RS2 and in the last estimate we applied Hölder's inequality.

Next, we verify relation (C.3.25). If $|\vec{x}| \geq 3(\rho+\tilde{\varepsilon})$, then $|\vec{y}-\vec{x}| \leq 2 \rho+3 \tilde{\varepsilon}$ implies $|\vec{y}| \geq \rho$ and the expression

$$
\tilde{F}_{\vec{x}}(\vec{p}):=(2 \pi)^{-\frac{s}{2}} \int d^{s} y e^{i \vec{p} \vec{y}} F(\vec{y}) \chi_{\vec{x}}\left(\mathcal{O}_{2(\rho+\tilde{\varepsilon})}\right)(\vec{y})
$$

defines a bounded, continuous function. The operator of multiplication by $\tilde{F}_{\vec{x}}$ in momentum space, denoted by the same symbol, satisfies the identity

$$
\chi\left(\mathcal{O}_{\rho}\right) \tilde{F}_{\vec{x}} \chi_{\vec{x}}\left(\mathcal{O}_{\rho}\right)=\chi\left(\mathcal{O}_{\rho}\right) \tilde{F} \chi_{\vec{x}}\left(\mathcal{O}_{\rho}\right) .
$$

To justify this equality of two bounded operators, it suffices to compare their matrix elements between $f_{1}, f_{2} \in S\left(\mathbb{R}^{s}\right)$. We introduce vectors $g_{1}=\chi\left(\mathcal{O}_{\rho}\right)^{*} f_{1}, g_{2}=\chi_{\vec{x}}\left(\mathcal{O}_{\rho}\right) f_{2}$ and compute

$$
\begin{array}{r}
\left\langle f_{1}\left|\chi\left(\mathcal{O}_{\rho}\right) \tilde{F} \chi_{\vec{x}}\left(\mathcal{O}_{\rho}\right)\right| f_{2}\right\rangle=\int d^{s} p \overline{\widetilde{g}}_{1}(\vec{p}) \tilde{F}(\vec{p}) \widetilde{g}_{2}(\vec{p})=\tilde{F}\left(\overline{\widetilde{g}}_{1} \widetilde{g}_{2}\right)=F\left((2 \pi)^{-\frac{s}{2}} \bar{g}_{1}^{\wedge} * g_{2}\right) \\
=\left(\chi_{\vec{x}}\left(\mathcal{O}_{2 \rho+2 \tilde{\varepsilon}}\right) F\right)\left((2 \pi)^{-\frac{s}{2}} \bar{g}_{1}^{\wedge} * g_{2}\right)=\left\langle f_{1}\left|\chi\left(\mathcal{O}_{\rho}\right) \tilde{F}_{\vec{x}} \chi_{\vec{x}}\left(\mathcal{O}_{\rho}\right)\right| f_{2}\right\rangle,
\end{array}
$$

where $g_{1}^{\wedge}(\vec{x})=g_{1}(-\vec{x})$, in the fourth step we made use of the support properties of $g_{1}$ and $g_{2}$ in configuration space and in the last step we reversed the first three steps with $F$ replaced with $\chi_{\vec{x}}\left(\mathcal{O}_{2 \rho+2 \tilde{\varepsilon}}\right) F$. Finally, we obtain from (C.3.27)

$$
\begin{array}{r}
\left|\tilde{F}_{\vec{x}}(\vec{p})\right| \leq(2 \pi)^{-\frac{s}{2}} \int d^{s} y\left|\chi\left(\mathcal{O}_{2(\rho+\tilde{\varepsilon})}\right)(\vec{y})\right| \sup _{|\vec{z}| \leq 2 \rho+3 \tilde{\varepsilon}}|F(\vec{z}+\vec{x})| \\
=c_{\rho, \tilde{\varepsilon}} \sup _{|\vec{z}| \leq 2 \rho+3 \tilde{\varepsilon}}|F(\vec{z}+\vec{x})| .
\end{array}
$$

\footnotetext{
${ }^{1}$ The Young inequality states that for any positive functions $f \in L^{r_{1}}\left(\mathbb{R}^{s}, d^{s} p\right), g \in L^{r_{2}}\left(\mathbb{R}^{s}, d^{s} p\right), h \in$ $L^{r_{3}}\left(\mathbb{R}^{s}, d^{s} p\right)$, where $1 \leq r_{1}, r_{2}, r_{3} \leq \infty$ s.t. $\frac{1}{r_{1}}+\frac{1}{r_{2}}+\frac{1}{r_{3}}=2$, there holds the bound

$$
\int d^{s} p d^{s} q f(\vec{p}) g(\vec{p}-\vec{q}) h(\vec{q}) \leq c_{r_{1}, r_{2}, r_{3}}\|f\|_{r_{1}}\|g\|_{r_{2}}\|h\|_{r_{3}} .
$$
}


From this bound and identity (C.3.28) there follows the estimate in the statement of the lemma.

We recall that $h_{r} \in D\left(\mathcal{O}_{r}\right)_{\mathbb{R}}$ has strictly positive Fourier transform and, by Lemma B.4.5 the operators $T_{h, \pm}=\omega^{-\gamma} \tilde{h}_{r}^{1 / 2} \mathcal{L}_{r}^{ \pm}$are bounded for any $\frac{1}{2} \leq \gamma<\frac{s-1}{2}$. Thus we can set in Lemma C.3.1 $h=\tilde{h}_{r}^{-1}, g_{k, i}=\omega^{-\frac{1}{2}} \tilde{h}_{r} \mathcal{L}_{r}^{ \pm} e_{i}$ for $k \in\{1,2\}$ and $i \in \mathbb{N}$, where $\left\{e_{i}\right\}_{1}^{\infty}$ is the orthonormal basis of eigenvectors of the operator $T$, given by (B.4.28). The resulting estimate, studied in Lemma C.3.8 below, relies on the decay properties of the functions

$$
\mathbb{R}^{s} \ni \vec{x} \rightarrow\left\langle\omega^{-\frac{1}{2}} \tilde{h}_{r} \mathcal{L}_{r}^{ \pm} e_{i} \mid U(\vec{x}) \omega^{-\frac{1}{2}} \tilde{h}_{r} \mathcal{L}_{r}^{ \pm} e_{i}\right\rangle
$$

which appear on the r.h.s. of relation (C.3.1). These properties are established in the following lemma with the help of the relation

$$
\mathcal{L}_{r}^{ \pm}=\omega^{\mp \frac{1}{2}} \chi\left(\mathcal{O}_{r}\right) \omega^{ \pm \frac{1}{2}} \mathcal{L}_{r}^{ \pm}
$$

which provides a link with Lemma C.3.6.

Lemma C.3.7. Assume that $s \geq 3$ and let e be a normalized eigenvector of the operator $T$, given by B.4.28), corresponding to the eigenvalue $t$. Then there holds

(a) $\left\langle\omega^{-\frac{1}{2}} \tilde{h}_{r} \mathcal{L}_{r}^{-} e \mid U(\vec{x}) \omega^{-\frac{1}{2}} \tilde{h}_{r} \mathcal{L}_{r}^{-} e\right\rangle=0$ for $|\vec{x}|>4 r$,

(b) $\left|\left\langle\omega^{-\frac{1}{2}} \tilde{h}_{r} \mathcal{L}_{r}^{ \pm} e \mid U(\vec{x}) \omega^{-\frac{1}{2}} \tilde{h}_{r} \mathcal{L}_{r}^{ \pm} e\right\rangle\right| \leq \frac{c_{r}(m) e^{-\frac{m|\vec{x}|}{2}} t^{2}}{(|\vec{x}|+1)^{s-2}}$,

where the constant $c_{r}(m)$ is independent of $\vec{x}$, $e$ and finite for any $m \geq 0$. (If $m>0$, the above relations hold for $s \geq 1$ ).

Proof. To prove part (a), we set again $\chi_{\vec{x}}\left(\mathcal{O}_{r}\right)(\vec{y})=\chi\left(\mathcal{O}_{r}\right)(\vec{y}-\vec{x})$ and note that

$$
\begin{aligned}
& \left\langle\omega^{-\frac{1}{2}} \tilde{h}_{r} \mathcal{L}_{r}^{-} e \mid U(\vec{x}) \omega^{-\frac{1}{2}} \tilde{h}_{r} \mathcal{L}_{r}^{-} e\right\rangle \\
& =\left\langle\omega^{-\frac{1}{2}} \tilde{h}_{r} \mathcal{L}_{r}^{-} e \mid \chi\left(\mathcal{O}_{2 r}\right) \chi_{\vec{x}}\left(\mathcal{O}_{2 r}\right) U(\vec{x}) \omega^{-\frac{1}{2}} \tilde{h}_{r} \mathcal{L}_{r}^{-} e\right\rangle=0,
\end{aligned}
$$

for $|\vec{x}|>4 r$, since $h_{r} \in D\left(\mathcal{O}_{r}\right)$ and hence $\omega^{-\frac{1}{2}} \tilde{h}_{r} \mathcal{L}_{r}^{-} e \in\left[\widetilde{D}\left(\mathcal{O}_{2 r}\right)\right]$. This latter statement follows from the fact that $\omega^{-\frac{1}{2}} \tilde{h}_{r} \mathcal{L}_{r}^{-} e=\left(\omega^{-\frac{1}{2}} \tilde{h}_{r} \mathcal{L}_{r}^{-}\right) \mathcal{L}_{r}^{-} e$ and $\omega^{-\frac{1}{2}} \tilde{h}_{r} \mathcal{L}_{r}^{-}$is a bounded operator due to Lemma B.4.5. In view of the uniform bound

$$
\left|\left\langle\omega^{-\frac{1}{2}} \tilde{h}_{r} \mathcal{L}_{r}^{ \pm} e \mid U(\vec{x}) \omega^{-\frac{1}{2}} \tilde{h}_{r} \mathcal{L}_{r}^{ \pm} e\right\rangle\right| \leq\left\|\omega^{\gamma-\frac{1}{2}} \tilde{h}_{r}^{1 / 2}\right\|_{\infty}^{2}\left\langle e \mid T_{h, \pm}^{2} e\right\rangle \leq\left\|\omega^{2 \gamma-1} \tilde{h}_{r}\right\|_{\infty} t^{2},
$$

which involves the parameter $\gamma \in\left[\frac{1}{2}, \frac{s-1}{2}\right.$ [ from the definition of the operator $T$, there also follows the $(-)$ part of $(\mathrm{b})$. To prove the $(+)$ part we estimate

$$
\begin{aligned}
\left|\left\langle\omega^{-\frac{1}{2}} \tilde{h}_{r} \mathcal{L}_{r}^{+} e \mid U(\vec{x}) \omega^{-\frac{1}{2}} \tilde{h}_{r} \mathcal{L}_{r}^{+} e\right\rangle\right| & =\left|\left\langle\tilde{h}_{r} \omega^{\frac{1}{2}} \mathcal{L}_{r}^{+} e \mid \chi\left(\mathcal{O}_{2 r}\right) \omega^{-2} \chi_{\vec{x}}\left(\mathcal{O}_{2 r}\right) \tilde{h}_{r} \omega^{\frac{1}{2}} U(\vec{x}) \mathcal{L}_{r}^{+} e\right\rangle\right| \\
& \leq t^{2}\left\|\omega^{2 \gamma+1} \tilde{h}_{r}\right\|_{\infty}\left\|\chi\left(\mathcal{O}_{2 r}\right) \omega^{-2} \chi_{\vec{x}}\left(\mathcal{O}_{2 r}\right)\right\| . \quad(\text { C.3. }
\end{aligned}
$$

Now we are in position to apply Lemma C.3.6. We set $\tilde{F}(\vec{p})=\left(|\vec{p}|^{2}+m^{2}\right)^{-1}, m \geq 0$ and obtain

$$
\tilde{F}(\vec{p})^{1 / 2}=\left(|\vec{p}|^{2}+m^{2}\right)^{-\frac{1}{2}} \theta(-|\vec{p}|+1)+\left(|\vec{p}|^{2}+m^{2}\right)^{-\frac{1}{2}} \theta(|\vec{p}|-1) \in L^{2}\left(\mathbb{R}^{s}, d^{s} p\right)+L^{\infty}\left(\mathbb{R}^{s}, d^{s} p\right) .
$$


For $s \geq 2, F(\vec{x})=\frac{1}{|\vec{x}|^{s-2}}(m|\vec{x}|)^{\frac{s-2}{2}} K_{\frac{s-2}{2}}(m|\vec{x}|)$, where $K_{\frac{s-2}{2}}$ is the modified Bessel function of the second kind. It satisfies the following bound for $s^{2} \geq 3$ and $z \geq 0$

$$
z^{\frac{s-2}{2}} K_{\frac{s-2}{2}}(z) \leq c e^{-\frac{z}{2}}
$$

where the constant $c$ is independent of $z$. (See Section 7.2 of [GJ] for a proof). Consequently, we obtain for $|\vec{x}| \geq 6 r+3 \tilde{\varepsilon}$

$$
\left\|\chi\left(\mathcal{O}_{2 r}\right) \omega^{-2} \chi_{\vec{x}}\left(\mathcal{O}_{2 r}\right)\right\| \leq \frac{c_{r} e^{-\frac{m}{2}(|\vec{x}|-4 r-3 \tilde{\varepsilon})}}{(|\vec{x}|-4 r-3 \tilde{\varepsilon})^{s-2}} .
$$

Making use of the uniform bound (C.3.34), we get the estimate from the statement of the lemma for a suitable constant $c_{r}(m)$ and $s \geq 3$.

It remains to consider the massive case in low dimensions. For $s=2$ there holds $F(\vec{x})=$ $K_{0}(m|\vec{x}|)$, what implies $|F(\vec{x})| \leq c_{r, m} e^{-m|\vec{x}|}$ for $|\vec{x}| \geq 2 r$. (See [GJ] Proposition 7.2.1. (c)). For $s=1$ we have the explicit formula $F(\vec{x})=\left((2 \pi)^{\frac{1}{2}} m\right)^{-1} e^{-m|\vec{x}|}$. In both cases we easily verify, by an analogous argument as above, that the estimate in part (b) of the lemma holds for $m>0$.

With our choice of the set $\mathcal{S}$, (see Section C.2), the map $\Theta_{E, 2}$, given by expansion (B.5.37), has the form

$$
\Theta_{E, 2}(A)=\sum_{\substack{\bar{\mu}, \bar{\nu} \\\left|\mu^{+}\right|+\left|\nu^{+}\right|>3 \text { or }\left|\mu^{-}\right|+\left|\nu^{-}\right|>0}} \tau_{\bar{\mu}, \bar{\nu}}(A) S_{\bar{\mu}, \bar{\nu}}
$$

We recall from formula (B.5.35) and the bound (B.5.36) that

$$
S_{\bar{\mu}, \bar{\nu}}=P_{E} a^{*}\left(\mathcal{L}_{r} e\right)^{\bar{\mu}} a\left(\mathcal{L}_{r} e\right)^{\bar{\nu}} P_{E}
$$

are bounded operators. In the following lemma we show that they are square-integrable under the restrictions on $\bar{\mu}, \bar{\nu}$ appearing in the sum (C.3.39).

Lemma C.3.8. Let $(\bar{\mu}, \bar{\nu})$ be a pair of 2-multiindices $(\bar{\mu}, \bar{\nu})$ s.t. $\left|\mu^{+}\right|+\left|\nu^{+}\right|>3$ or $\left|\mu^{-}\right|+\left|\nu^{-}\right|>0$. Then, in massless scalar free field theory for $s \geq 3$, there holds the bound

$$
\left\|S_{\bar{\mu}, \bar{\nu}}\right\|_{E, 2} \leq C_{r, E} E^{\frac{|\bar{\mu}|+|\bar{\nu}|}{2}} t^{\bar{\mu}} t^{\bar{\nu}}
$$

where $\left\{t_{j}\right\}_{1}^{\infty}$ are the eigenvalues of the operator $T$ given by B.4.28) and the constant $C_{r, E}$ is independent of $\bar{\mu}, \bar{\nu}$.

Proof. Any pair of 2-multiindices $(\bar{\mu}, \bar{\nu})$ satisfying the restrictions from the statement of the lemma can be decomposed as follows:

$$
\begin{aligned}
& \bar{\mu}=\bar{\mu}_{a}+\bar{\mu}_{b}, \\
& \bar{\nu}=\bar{\nu}_{a}+\bar{\nu}_{b},
\end{aligned}
$$

where $\left|\bar{\mu}_{a}\right|+\left|\bar{\nu}_{a}\right|=\left|\mu_{a}^{+}\right|+\left|\nu_{a}^{+}\right|=4$ or $\left|\bar{\mu}_{a}\right|+\left|\bar{\nu}_{a}\right|=\left|\mu_{a}^{-}\right|+\left|\nu_{a}^{-}\right|=1$. For any compact 
subset $K \subset \mathbb{R}^{s}$ and $\varphi \in \mathcal{T}_{E, 1}^{+}$we obtain

$$
\begin{array}{r}
\int_{K} d^{s} x\left|\varphi\left(S_{\bar{\mu}, \bar{\nu}}(\vec{x})\right)\right|^{2} \leq \int_{K} d^{s} x \varphi_{\vec{x}}\left(a^{*}\left(\mathcal{L}_{r} e\right)^{\bar{\mu}} a\left(\mathcal{L}_{r} e\right)^{\bar{\mu}}\right) \varphi_{\vec{x}}\left(a^{*}\left(\mathcal{L}_{r} e\right)^{\bar{\nu}} a\left(\mathcal{L}_{r} e\right)^{\bar{\nu}}\right) \\
=\int_{K} d^{s} x \varphi_{\vec{x}}\left(a^{*}\left(\mathcal{L}_{r} e\right)^{\bar{\mu}_{a}} a^{*}\left(\mathcal{L}_{r} e\right)^{\bar{\mu}_{b}} a\left(\mathcal{L}_{r} e\right)^{\bar{\mu}_{b}} a\left(\mathcal{L}_{r} e\right)^{\bar{\mu}_{a}}\right) \\
\cdot \varphi_{\vec{x}}\left(a^{*}\left(\mathcal{L}_{r} e\right)^{\bar{\nu}_{a}} a^{*}\left(\mathcal{L}_{r} e\right)^{\bar{\nu}_{b}} a\left(\mathcal{L}_{r} e\right)^{\bar{\nu}_{b}} a\left(\mathcal{L}_{r} e\right)^{\bar{\nu}_{a}}\right) \\
\leq E^{\left|\bar{\mu}_{b}\right|+\left|\bar{\nu}_{b}\right|} t^{2 \bar{\mu}_{b}} t^{2 \bar{\nu}_{b}} \int_{K} d^{s} x \varphi_{\vec{x}}\left(a^{*}\left(\mathcal{L}_{r} e\right)^{\bar{\mu}_{a}} a\left(\mathcal{L}_{r} e\right)^{\bar{\mu}_{a}}\right) \\
\cdot \varphi_{\vec{x}}\left(a^{*}\left(\mathcal{L}_{r} e\right)^{\bar{\nu}_{a}} a\left(\mathcal{L}_{r} e\right)^{\bar{\nu}_{a}}\right),
\end{array}
$$

where in the first step we made use of the Cauchy-Schwarz inequality and in the third step we used estimate (B.5.36). Now we set in Lemma C.3.1 $h=\tilde{h}_{r}^{-1}, g_{k, i}=\omega^{-\frac{1}{2}} \tilde{h}_{r} \mathcal{L}_{r}^{ \pm} e_{i}$ for $k \in\{1,2\}, i \in \mathbb{N}$, where the function $\tilde{h}_{r}>0$ entered into the definition (B.4.28) of the operator $T$. We obtain

$$
\begin{aligned}
& \sup _{\varphi \in \mathcal{T}_{E, 1}^{+}} \int_{K} d^{s} x\left|\varphi\left(S_{\bar{\mu}, \bar{\nu}}(\vec{x})\right)\right|^{2} \leq E^{|\bar{\mu}|+|\bar{\nu}|} t^{2 \bar{\mu}_{b}} t^{2 \bar{\nu}_{b}}\left(\sup _{\omega(\vec{p}) \leq E}\left|\tilde{h}_{r}(\vec{p})\right|^{-2}\right)^{\left|\bar{\mu}_{a}\right|+\left|\bar{\nu}_{a}\right|} \\
& \cdot \int_{\Delta K} d^{s} y\left|\left(\Omega \mid b\left(\tilde{h}_{r} \omega^{-\frac{1}{2}} \mathcal{L}_{r} e\right)^{\bar{\mu}_{a}} b^{*}\left(U(\vec{y}) \tilde{h}_{r} \omega^{-\frac{1}{2}} \mathcal{L}_{r} e\right)^{\bar{\mu}_{a}} \Omega\right)\right| \\
& \cdot\left|\left(\Omega \mid b\left(\tilde{h}_{r} \omega^{-\frac{1}{2}} \mathcal{L}_{r} e\right)^{\bar{\nu}_{a}} b^{*}\left(U(\vec{y}) \tilde{h}_{r} \omega^{-\frac{1}{2}} \mathcal{L}_{r} e\right)^{\bar{\nu}_{a}} \Omega\right)\right| \\
& \leq E^{|\bar{\mu}|+|\bar{\nu}|} t^{2 \bar{\mu}_{b}} t^{2 \bar{\nu}_{b}}\left(\sup _{\omega(\vec{p}) \leq E}\left|\tilde{h}_{r}(\vec{p})\right|^{-2}\right)^{\left|\bar{\mu}_{a}\right|+\left|\bar{\nu}_{a}\right|} \\
& \cdot \int_{\Delta K} d^{s} y\left|\left\langle\tilde{h}_{r} \omega^{-\frac{1}{2}} \mathcal{L}_{r} e \mid U(\vec{y}) \omega^{-\frac{1}{2}} \tilde{h}_{r} \mathcal{L}_{r} e\right\rangle^{\bar{\mu}_{a}}\right| \cdot\left|\left\langle\tilde{h}_{r} \omega^{-\frac{1}{2}} \mathcal{L}_{r} e \mid U(\vec{y}) \tilde{h}_{r} \omega^{-\frac{1}{2}} \mathcal{L}_{r} e\right\rangle^{\bar{\nu}_{a}}\right| \\
& \leq C_{r, E}^{2} E^{|\bar{\mu}|+|\bar{\nu}|} t^{2 \bar{\mu}} t^{2 \bar{\nu}}
\end{aligned}
$$

We note that the integral, obtained in the second step, converges with $\Delta K \nearrow \mathbb{R}^{s}$ due to the properties of the 2-multiindices $\bar{\nu}_{a}, \bar{\mu}_{a}$ and Lemma C.3.7. Thus the constant $C_{r, E}$ can be chosen independently of $K$ and the above bound still holds after replacing $K$ with $\mathbb{R}^{s}$. Now the estimate in the statement of the lemma follows from the bound (C.3.16) after readjusting the constant $C_{r, E}$.

Now we are ready to prove the main result of this subsection.

Proposition C.3.9. In massless scalar free field theory for $s \geq 3$ the map $\Theta_{E, 2}$, given by relation (C.3.39), satisfies the bound

$$
\sup _{A \in \mathfrak{A}(\mathcal{O}(r))_{1}}\left\|\Theta_{E, 2}(A)\right\|_{E, 2}<\infty
$$

Proof. First, we recall that the bound (B.5.34) gives $\left\|\tau_{\bar{\mu}, \bar{\nu}}\right\| \leq 2^{\frac{5}{2}(|\bar{\mu}|+|\bar{\nu}|)}(\bar{\mu} ! \bar{\nu} !)^{-\frac{1}{2}}$. Next, 
making use of the Minkowski inequality (C.3.11), we obtain for any $A \in \mathfrak{A}(\mathcal{O}(r))_{1}$

$$
\begin{aligned}
\left\|\Theta_{E, 2}(A)\right\|_{E, 2} & \leq \sum_{\substack{\bar{\mu}, \bar{\nu} \\
\left|\mu^{+}\right|+\left|\nu^{+}\right|>3 \text { or }\left|\mu^{-}\right|+\left|\nu^{-}\right|>0}}\left\|\tau_{\bar{\mu}, \bar{\nu} \|}\right\| S_{\bar{\mu}, \bar{\nu} \|} \|_{E, 2} \leq C_{r} \sum_{\bar{\mu}, \bar{\nu}} \frac{\left(2^{5} E\right)^{\frac{|\bar{\mu}|+|\bar{\nu}|}{2}}}{(\bar{\mu} ! \bar{\nu} !)^{\frac{1}{2}}} t^{\bar{\mu}} t^{\bar{\nu}} \\
& \left.=C_{r}\left(\sum_{\mu^{+}} \frac{\left(2^{5} E\right)^{\frac{\left|\mu^{+}\right|}{2}}}{\left(\mu^{+} !\right)^{\frac{1}{2}}} t^{\mu^{+}}\right)^{4} \leq C_{r}\left(\sum_{k=0}^{\infty} \frac{\left(2^{5} E\|T\|_{1}^{2}\right)^{\frac{k}{2}}}{k !^{\frac{1}{2}}}\right)^{4}, \quad \text { C.3.4 }\right)
\end{aligned}
$$

where in the second step we applied Lemma C.3.8 and in the last step we made use of the fact that the multinomial coefficients are larger or equal to one, and of the multinomial formula (B.1.4). Clearly, the last expression on the r.h.s. of the above estimate is finite.

From relation (C.2.22) and Propositions C.3.5 C.3.9 we conclude that for any $r>0$, $E \geq 0$ and $A \in \mathfrak{A}(\mathcal{O}(r))$ there holds

$$
\left\|R^{(2)}(A)\right\|_{E, 2}<\infty
$$

what completes the proof of Theorem C.1.1 Thus we have verified that Condition $L^{(2)}$ holds in massless scalar free field theory, its even part and its sub-theory generated by the derivatives of the field for $s \geq 3$. 


\section{Appendix D}

\section{Verification of Condition $L_{\sharp}^{(1)}$ in Massive Scalar Free Field Theory}

In this appendix we verify that Condition $L_{\sharp}^{(1)}$, stated in Section 2.3. holds in massive free field theory. In Section D.1 we recall the relevant background material from Appendix B. In Section D.2 we verify Condition $L^{(1)}$. Section D.3 is devoted to its strengthened variant.

\section{D.1 Preliminaries}

We infer from Propositions B.5.4 and B.5.5 that in massive free field theory the map $\Theta_{E}: \mathfrak{A}(\mathcal{O}(r)) \rightarrow B(\mathcal{H})$, given by $\Theta_{E}(A)=P_{E} A P_{E}$, has the following expansions for $A \in \mathfrak{A}_{\mathrm{c}}(\mathcal{O}(r))$,

$$
\begin{aligned}
& \Theta_{E}(A)=\sum_{\substack{\bar{\mu}, \bar{\nu} \\
|\bar{\mu}|+|\bar{\nu}| \neq 0}} \stackrel{\vee}{\tau}_{\bar{\mu}, \bar{\nu}}^{(r)}(A) \stackrel{\vee}{S_{\bar{\mu}}, \bar{\nu}}, \\
& \Theta_{E}(A)=\sum_{\substack{\bar{\mu}, \bar{\nu} \\
|\bar{\mu}|+|\bar{\nu}| \neq 0}} \tau_{\bar{\mu}, \bar{\nu}}(A) S_{\bar{\mu}, \bar{\nu}},
\end{aligned}
$$

which converge in the norm topology in $B(\mathcal{H})$. Here we made use of the fact that $\stackrel{\vee}{\tau_{0,0}}=$ $\tau_{0,0}=\omega_{0}$. By definitions (B.5.19) and (B.5.35), the operators $\stackrel{\vee}{S}{ }_{\bar{\mu}, \bar{\nu}}, S_{\bar{\mu}, \bar{\nu}}$ have the form

$$
\begin{aligned}
& \stackrel{\vee}{S}_{\bar{\mu}, \bar{\nu}}=P_{E} a^{*}\left(h_{\kappa, E}\right)^{\bar{\mu}} a\left(h_{\kappa, E}\right)^{\bar{\nu}} P_{E}, \\
& S_{\bar{\mu}, \bar{\nu}}=P_{E} a^{*}\left(\mathcal{L}_{r} e\right)^{\bar{\mu}} a\left(\mathcal{L}_{r} e\right)^{\bar{\nu}} P_{E},
\end{aligned}
$$

where the vectors $\left\{\tilde{h}_{\kappa_{j}, E}^{ \pm}\right\}_{1}^{\infty}$ are defined by (B.4.2) and $\left\{e_{j}\right\}_{1}^{\infty}$ are the eigenvectors of the operator $T$, given by (B.4.28), whose eigenvalues are denoted by $\left\{t_{j}\right\}_{1}^{\infty}$. Due to the bounds (B.5.23) and (B.5.36) there holds

$$
\begin{aligned}
&\left\|S_{\bar{\mu}, \bar{\nu}}^{\vee}\right\| \leq E^{\frac{|\bar{\mu}|+|\bar{\nu}|}{2}}\left\|\omega^{-\frac{1}{2}} \tilde{h}_{\kappa, E}\right\|_{2}^{\bar{\mu}+\bar{\nu}} \leq M_{E}^{\frac{|\bar{\mu}|+|\bar{\nu}|}{2}}\left\|\tilde{h}_{\kappa, E}\right\|_{2}^{\bar{\mu}+\bar{\nu}}, \\
&\left\|S_{\bar{\mu}, \bar{\nu}}\right\| \leq E^{\frac{|\bar{\mu}|+|\bar{\nu}|}{2}} t^{\bar{\mu}} t^{\bar{\nu}}
\end{aligned}
$$


where in the second step of the first estimate we made use of the fact that in the massive theory $\left\|\omega^{-\frac{1}{2}}\right\|=m^{-\frac{1}{2}}$ and we set $M_{E}=\frac{E}{m}$. We note that $\stackrel{\vee}{S}{ }_{\bar{\mu}, \bar{\nu}}=S_{\bar{\mu}, \bar{\nu}}=0$ for $|\bar{\mu}|>\left[M_{E}\right]$ or $|\bar{\nu}|>\left[M_{E}\right]$. Thus, by Lemma B.5.1, the normal functionals $\stackrel{\vee}{\tau_{\bar{\mu}}(r)}, \tau_{\bar{\mu}, \bar{\nu}}$, entering into the sums (D.1.1), (D.1.2), satisfy the following estimates for any $l \geq 0$

$$
\begin{aligned}
\left\|R^{-l} \tau_{\bar{\mu}, \bar{\nu}}^{\vee(r)} R^{-l}\right\| & \leq\left(2 c_{l}\right)^{2 M_{E}} \sqrt{\left(2\left[M_{E}\right]\right) !}\left\|b_{\kappa, r}\right\|_{2, l}^{\bar{\mu}+\bar{\nu}}, \\
\left\|\tau_{\bar{\mu}, \bar{\nu}}\right\| & \leq \frac{2^{\frac{5}{2}(|\bar{\mu}|+|\bar{\nu}|)}}{(\bar{\mu} ! \bar{\nu} !)^{\frac{1}{2}}} \leq 2^{5 M_{E}},
\end{aligned}
$$

where $c_{l}=\left(12+2 m^{2}\right)^{l / 2}$, the vectors $\left\{b_{\kappa_{j}, r}^{ \pm}\right\}_{1}^{\infty}$ are defined by (B.4.1) and the Sobolev norms are given by (B.3.2). Finally, by Lemmas B.4.3 and B.4.4 we obtain the following bound for any combination of \pm -signs and any $\lambda, l \geq 0$

$$
\sum_{\kappa \in \mathbb{N}_{0}^{s}}\left\|b_{\kappa, r}^{ \pm}\right\|_{2, l}\left\|\tilde{h}_{\kappa, E}^{ \pm}\right\|_{2, \lambda} \leq \sum_{\kappa \in \mathbb{N}_{0}^{s}} \frac{\left(c_{l, r} c_{\lambda, 0, E}\right)^{(|\kappa|+1)}}{\kappa !}<\infty .
$$

This concludes the list of auxiliary results which we need to verify Condition $L_{\sharp}^{(1)}$.

\section{D.2 Verification of Condition $L^{(1)}$}

Let us first briefly describe our strategy: By Theorem 1.6.1 observables of the form $B^{*} B$, where $B \in \mathfrak{A}$ is almost local and energy-decreasing, are integrable i.e. they belong to $\mathfrak{A}^{(1)}$. The operators $\stackrel{\vee}{S}_{\bar{\mu}, \bar{\nu}}$ are of similar form for $|\bar{\mu}| \neq 0$ and $|\bar{\nu}| \neq 0$. In fact, we will show in Lemma D.2.2 below that under such restriction $\left\|\stackrel{v}{S}_{\bar{\mu}, \bar{\nu}}\right\|_{E, 1}<\infty$. The role of the time-smearing function $g$, entering into Condition $L_{\sharp}^{(1)}$, is to eliminate all other terms.

Lemma D.2.1. Let $g \in S(\mathbb{R})$ be s.t. supp $\widetilde{g} \subset]-m, m[$, where $m>0$ is the mass of free field theory. Let $n \geq 1$ and $h_{1}, \ldots, h_{n} \in L^{2}\left(\mathbb{R}^{s}, d^{s} p\right)$. Then

$$
P_{E}\left(a\left(h_{1}\right) \ldots a\left(h_{n}\right)\right)(g) P_{E}=0 .
$$

In particular, if $|\bar{\mu}|=0$ or $|\bar{\nu}|=0$ but $|\bar{\mu}|+|\bar{\nu}| \neq 0$ then $\stackrel{\vee}{S}_{\bar{\mu}, \bar{\nu}}(g)=S_{\bar{\mu}, \bar{\nu}}(g)=0$.

Proof. Let $\Psi_{1}, \Psi_{2} \in P_{E} \mathcal{H} \cap D_{S}$. Then we obtain

$$
\begin{aligned}
& \left(\Psi_{1} \mid P_{E}\left(a\left(h_{1}\right) \ldots a\left(h_{n}\right)\right)(g) P_{E} \Psi_{2}\right) \\
= & \int d t g(t) \int d^{s} p_{1} \ldots d^{s} p_{n} \bar{h}\left(\vec{p}_{1}\right) e^{-i \omega\left(\vec{p}_{1}\right) t} \ldots \bar{h}\left(\vec{p}_{n}\right) e^{-i \omega\left(\vec{p}_{n}\right) t}\left(\Psi_{1} \mid a\left(\vec{p}_{1}\right) \ldots a\left(\vec{p}_{n}\right) \Psi_{2}\right) \\
= & (2 \pi)^{\frac{1}{2}} \int d^{s} p_{1} \ldots d^{s} p_{n} \widetilde{g}\left(-\left(\omega\left(\vec{p}_{1}\right)+\cdots+\omega\left(\vec{p}_{n}\right)\right)\right) \\
\cdot & \left.\bar{h}\left(\vec{p}_{1}\right) \ldots \bar{h}\left(\vec{p}_{n}\right)\left(\Psi_{1} \mid a\left(\vec{p}_{1}\right) \ldots a\left(\vec{p}_{n}\right) \Psi_{2}\right)=0, \quad \text { D } .2 .2\right)
\end{aligned}
$$

where in the last step we exploited the support properties of $\widetilde{g}$. Making use of the energy bounds (B.5.22) and the fact that $\left\|\omega^{-\frac{1}{2}}\right\|<\infty$ in the massive theory, we conclude that

$$
P_{E}\left(a\left(h_{1}\right) \ldots a\left(h_{n}\right)\right)(g) P_{E}=0 .
$$


Given the restrictions on $(\bar{\mu}, \bar{\nu})$ in the statement of the lemma, either $\stackrel{\vee}{S}_{\bar{\mu}, \bar{\nu}}(g)$ or $\left(\stackrel{\vee}{S}_{\bar{\mu}, \bar{\nu}}(\bar{g})\right)^{*}$ is of the form considered above. The same is true for $S_{\bar{\mu}, \bar{\nu}}$.

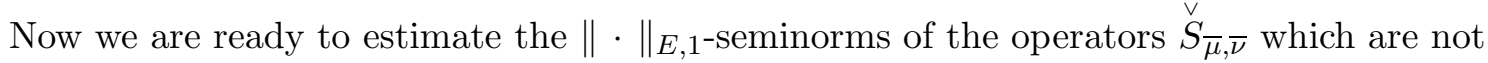
covered by the above lemma. We will exploit the fact that for any $C \in B(\mathcal{H})$, which satisfies $\|C\|_{E, 1}<\infty$, there holds

$$
\|C\|_{E, 1} \leq 4 \sup _{\varphi \in \mathcal{T}_{E, 1}^{+}} \int d^{s} x|\varphi(C(\vec{x}))| .
$$

This bound follows immediately from decomposition (1.6.2).

Lemma D.2.2. Let $(\bar{\mu}, \bar{\nu})$ be a pair of 2-multiindices s.t. $|\bar{\mu}| \cdot|\bar{\nu}| \neq 0$ and $\lambda>\frac{s}{2}$. Then there holds

$$
\left\|\stackrel{\vee}{S}_{\bar{\mu}, \bar{\nu}}\right\|_{E, 1} \leq c_{\lambda} M_{E}^{\frac{|\bar{\mu}|+|\bar{\nu}|}{2}}\left\|\tilde{h}_{\kappa, E}\right\|_{2, \lambda}^{\bar{\mu}+\bar{\nu}},
$$

where $M_{E}=\frac{E}{m}$ and $c_{\lambda}$ is a constant independent of $\bar{\mu}, \bar{\nu}$.

Proof. For any compact subset $K \subset \mathbb{R}^{s}$ we obtain from the Cauchy-Schwarz inequality

$$
\begin{aligned}
& \sup _{\varphi \in \mathcal{T}_{E, 1}^{+}} \int_{K} d^{s} x\left|\varphi\left(\stackrel{\vee}{S}_{\bar{\mu}, \bar{\nu}}(\vec{x})\right)\right| \\
\leq & \sup _{\varphi \in \mathcal{T}_{E, 1}^{+}}\left(\int_{K} d^{s} x\left|\varphi\left(\stackrel{\vee}{S}_{\bar{\mu}, \bar{\mu}}(\vec{x})\right)\right|\right)^{\frac{1}{2}} \sup _{\varphi \in \mathcal{T}_{E, 1}^{+}}\left(\int_{K} d^{s} x\left|\varphi\left(\stackrel{\vee}{S}_{\bar{\nu}, \bar{\nu}}(\vec{x})\right)\right|\right)^{\frac{1}{2}} .
\end{aligned}
$$

We decompose $\bar{\mu}$ into two 2 -multiindices $\bar{\mu}=\bar{\mu}_{a}+\bar{\mu}_{b}$ in such a way that $\left|\bar{\mu}_{b}\right|=1$.

$$
\begin{aligned}
\int_{K} d^{s} x\left|\varphi\left(\stackrel{\vee}{S}_{\bar{\mu}, \bar{\mu}}(\vec{x})\right)\right| & =\int_{K} d^{s} x\left|\varphi\left(\left(a^{*}\left(h_{\kappa, E}\right)^{\bar{\mu}_{b}} P_{E} a^{*}\left(h_{\kappa, E}\right)^{\bar{\mu}_{a}} a\left(h_{\kappa, E}\right)^{\bar{\mu}_{a}} P_{E} a\left(h_{\kappa, E}\right)^{\bar{\mu}_{b}}\right)(\vec{x})\right)\right| \\
& \leq\left\|a\left(h_{\kappa, E}\right)^{\bar{\mu}_{a}} P_{E}\right\|^{2} \int_{K} d^{s} x \varphi\left(\left(a^{*}\left(h_{\kappa, E}\right)^{\bar{\mu}_{b}} a\left(h_{\kappa, E}\right)^{\bar{\mu}_{b}}\right)(\vec{x})\right) \\
& \leq\left\|\tilde{h}_{\kappa, E}\right\|_{2}^{2 \bar{\mu}_{a}} M_{E}^{|\bar{\mu}|} \int_{\Delta K} d^{s} x\left|\left\langle\tilde{h}_{\kappa, E}^{\bar{\mu}_{b}} \mid U(\vec{x}) \tilde{h}_{\kappa, E}^{\bar{\mu}_{b}}\right\rangle\right| \\
& \leq c_{\lambda} M_{E}^{|\bar{\mu}|}\left\|\tilde{h}_{\kappa, E}\right\|_{2, \lambda}^{2 \bar{\mu}} .
\end{aligned}
$$

Here in the third step we applied the bound (D.1.5 and Lemma C.3.1 with $h=\omega^{-\frac{1}{2}}$. In the last step we made use of Lemma C.3.3 and of the fact that $\|f\|_{2} \leq\|f\|_{2, \lambda}$ for any $f \in L^{2}\left(\mathbb{R}^{s}, d^{s} p\right)_{\lambda}$. The second factor on the r.h.s. of relation (D.2.6) satisfies an analogous bound. Thus we obtain

$$
\sup _{\varphi \in \mathcal{T}_{E, 1}^{+}} \int_{K} d^{s} x\left|\varphi\left(\stackrel{\vee}{S}_{\bar{\mu}, \bar{\nu}}(\vec{x})\right)\right| \leq M_{E}^{\frac{|\bar{\mu}|+|\bar{\nu}|}{2}} c_{\lambda}\left\|\tilde{h}_{\kappa, E}\right\|_{2, \lambda}^{\bar{\mu}+\bar{\nu}}
$$

Making use of the bound (D.2.4), redefining the constant $c_{\lambda}$ and taking the limit $K \nearrow \mathbb{R}^{s}$, we obtain the estimate in the statement of the lemma.

Next, we note that if $C \in B(\mathcal{H})$ satisfies $\|C\|_{E, 1}<\infty$ and $g \in S(\mathbb{R})$ is a time-smearing function then there holds

$$
\|C(g)\|_{E, 1} \leq\|g\|_{1}\|C\|_{E, 1}
$$


This bound is a simple consequence of the Fubini theorem. It concludes the list of auxiliary results needed to verify Condition $L^{(1)}$. Now we are ready to show that for any $r>0$ there holds the bound

$$
\|A(g)\|_{E, 1} \leq c_{l, E, r}\left\|R^{l} A R^{l}\right\|, \quad A \in \mathfrak{A}_{\mathrm{c}}(\mathcal{O}(r))
$$

where $g$ is defined as in the statement of Lemma D.2.1 and the constant $c_{l, E, r}$ is independent of $A$.

Theorem D.2.3. Condition $L^{(1)}$ holds in massive scalar free field theory for any dimension of space $s \geq 1$.

Proof. Let $g \in S(\mathbb{R})$ be s.t. $\operatorname{supp} \widetilde{g} \subset]-m, m[$ and $l \geq 0$. Making use of relation (D.1.1), we obtain

$$
\begin{aligned}
\|A(g)\|_{E, 1} \leq & \left\|R^{l} A R^{l}\right\| \sum_{\substack{\bar{\mu}, \bar{\nu} \\
|\bar{\mu}|+|\bar{\nu}| \neq 0}}\left\|R^{-l} \tau_{\bar{\mu}, \bar{\nu}}^{\vee(r)} R^{-l}\right\|\left\|\stackrel{S}{S}_{\bar{\mu}, \bar{\nu}}(g)\right\|_{E, 1} \\
\leq & \left\|R^{l} A R^{l}\right\|\|g\|_{1} c_{\lambda}\left(4 c_{l}^{2} M_{E}\right)^{M_{E}} \sqrt{\left(2\left[M_{E}\right]\right) !} \sum_{\bar{\mu}, \bar{\nu}}\left(\left\|b_{\kappa, r}\right\|_{2, l}\left\|\tilde{h}_{\kappa, E}\right\|_{2, \lambda}\right)^{\bar{\mu}+\bar{\nu}} \\
\leq & \left\|R^{l} A R^{l}\right\|\|g\|_{1} c_{\lambda}\left(4 c_{l}^{2} M_{E}\right)^{M_{E}} \sqrt{\left(2\left[M_{E}\right]\right) !} \\
& \cdot\left(\sum_{n_{1}=0}^{\left[M_{E}\right]}\left(\sum_{\kappa \in \mathbb{N}_{0}^{s}}\left\|b_{\kappa, r}^{+}\right\|_{2, l}\left\|\tilde{h}_{\kappa, E}^{+}\right\|_{2, \lambda}\right)^{n_{1}}\right)^{2}\left(\sum_{n_{2}=0}^{\left[M_{E}\right]}\left(\sum_{\kappa \in \mathbb{N}_{0}^{s}}\left\|b_{\kappa, r}^{-}\right\|_{2, l}\left\|\tilde{h}_{\kappa, E}^{-}\right\|_{2, \lambda}\right)^{n_{2}}\right)^{2} .
\end{aligned}
$$

Here in the second step we exploited estimate (D.1.7), Lemmas D.2.1 and D.2.2, and relation (D.2.9). In the last step we made use of the fact that the multinomial coefficients are larger or equal to one and of the multinomial formula (B.1.4). The last expression is finite by estimate (D.1.9).

We conclude this section with a brief comment on the even part of massive scalar free field theory $\left(\underline{\mathfrak{A}}^{(\mathrm{e})}, \underline{\alpha}, \mathcal{H}^{(\mathrm{e})}\right)$, introduced in Section B.2. There clearly holds for any $\underline{A} \in \underline{\mathfrak{A}}_{\mathrm{c}}^{(\mathrm{e})}(\mathcal{O})$ and $g \in S(\mathbb{R})$ as specified in Condition $L^{(1)}$

$$
\|\underline{A}(g)\|_{E, 1} \leq\left\|\pi_{(\mathrm{e})}^{-1}(\underline{A})(g)\right\|_{E, 1} \leq c_{0}\left\|\pi_{(\mathrm{e})}^{-1}(\underline{A})\right\|=c_{0}\|\underline{A}\|
$$

Here in the first step we made use of formula $(\underline{B .2 .18})$ and relation $(\underline{B .2 .20})$, in the second step we exploited Condition $L^{(1)}$ (for $l=0$ ), which is valid in (full) massive scalar free field theory by Theorem D.2.3 and in the last step we applied equality (B.2.17). We obtain:

Corollary D.2.4. Condition $L^{(1)}$ (a) holds in the even part of massive scalar free field theory $\left(\underline{\mathfrak{A}}^{(e)}, \underline{\alpha}, \mathcal{H}^{(e)}\right)$ for any dimension of space $s \geq 1$.

We conjecture that this model satisfies part (b) of Condition $L^{(1)}$ as well, but we do not have a complete argument at the moment. 


\section{D.3 Verification of Condition $L_{\sharp}^{(1)}$}

In this section we verify the strengthened variant of Condition $L^{(1)}$. In addition to the bound (D.2.10) above, we have to show that the operators $A(g)$, where $A \in \hat{\mathfrak{A}}_{\mathrm{c}}$ and $\widetilde{g}$ is supported in ] $-m, m[$, can be approximated by elements from $\mathfrak{C}(g)=\{C(g) \mid C \in \mathfrak{C}\}$ in the $T^{(1)}$-topology introduced in Section 2.3. In view of relation (2.3.19) we have to find, for any $E \geq 0$ and $\varepsilon>0$, an observable $C \in \mathfrak{C}$ s.t.

$$
\|A(g)-C(g)\|_{E, 1} \leq \varepsilon
$$

We will accomplish this task in the last part of this section. (See relations (D.3.16) and (D.3.17) below). The key observation is that in massive scalar free field theory, for $|\bar{\mu}| \cdot|\bar{\nu}| \neq 0$, there holds

$$
S_{\bar{\mu}, \bar{\nu}}=P_{E} B_{\bar{\mu}}^{*} B_{\bar{\nu}} P_{E}
$$

where $S_{\bar{\mu}, \bar{\nu}}$ is given by (D.1.4) and $B_{\bar{\mu}}, B_{\bar{\nu}} \in \mathfrak{A}$ are energy-decreasing and almost local observables which depend on $E$. This fact is established in the following lemma.

Lemma D.3.1. Let $s \geq 1$ and $e \in L^{2}\left(\mathbb{R}^{s}, d^{s} p\right)$ be s.t. Je $=e$. Then, in massive scalar free field theory, for any $E \geq 0$ there exist operators $B_{E}^{ \pm} \in \mathfrak{A}$ which are almost local, energy-decreasing and s.t. $a\left(\mathcal{L}_{r}^{ \pm} e\right) P_{E}=B_{E}^{ \pm} P_{E}$.

Proof. We consider only the $(+)$ case as the $(-)$ case is analogous. Without loss of generality we can assume that $\left[M_{E}\right]>1$ and $\left\|\mathcal{L}_{r}^{+} e\right\| \neq 0$. We pick a function $h \in S(\mathbb{R})$ which satisfies, for $n \in\left\{0,1, \ldots, 2\left[M_{E}\right]\right\}$,

$$
\int d u e^{-\frac{1}{2} u^{2}\left\|\mathcal{L}_{r}^{+} e\right\|^{2}} u^{n} h(u)=-i \delta_{n, 1} .
$$

Such function can be constructed as follows: Let $f_{n}(u)=e^{-\frac{1}{2} u^{2}\left\|\mathcal{L}_{r}^{+} e\right\|^{2}} u^{n}$. In the subspace $X=\operatorname{Span}\left\{f_{0}, f_{2}, \ldots, f_{2\left[M_{E}\right]}\right\}$ of $L^{2}(\mathbb{R}, d u)$ we can construct, with the help of the GramSchmidt procedure, an orthonormal basis $\left\{g_{0}, g_{2}, \ldots, g_{2\left[M_{E}\right]}\right\}$ in $X$, consisting of Schwartzclass functions. We introduce the projection on $X$

$$
\left.P_{X}=\mid g_{0}\right)\left(g_{0}|+| g_{2}\right)\left(g_{2}|+\cdots+| g_{2\left[M_{E}\right]}\right)\left(g_{2\left[M_{E}\right]} \mid\right.
$$

and note that $\left(I-P_{X}\right) f_{1} \neq 0$, since the functions $\left\{f_{n}\right\}_{1}^{2\left[M_{E}\right]}$ are linearly independent. We set

$$
h=-i \frac{\left(I-P_{X}\right) f_{1}}{\left\|\left(I-P_{X}\right) f_{1}\right\|^{2}}
$$

It is manifestly a Schwartz-class function which satisfies (D.3.3).

Next, we note that $e^{i u\left(a\left(\mathcal{L}_{r}^{+} e\right)+a^{*}\left(\mathcal{L}_{r}^{+} e\right)\right)} \in \mathfrak{A}(\mathcal{O}(r))$ for any $u \in \mathbb{R}$. (In fact, there exists a sequence $f_{n}^{+} \in(1+J) \dot{\mathcal{L}}_{r}^{+}$s.t. $\lim _{n \rightarrow \infty}\left\|f_{n}^{+}-\mathcal{L}_{r}^{+} e\right\|_{2}=0$. Thus, by Theorem X.41 (d) of [RS2], the corresponding sequence of Weyl operators $W\left(u f_{n}\right)$ converges to $e^{i u\left(a\left(\mathcal{L}_{r}^{+} e\right)+a^{*}\left(\mathcal{L}_{r}^{+} e\right)\right)}$ in the strong operator topology). Also the weak integral

$$
A=\int d u e^{i u\left(a\left(\mathcal{L}_{r}^{+} e\right)+a^{*}\left(\mathcal{L}_{r}^{+} e\right)\right)} h(u)
$$


defines an element of $\mathfrak{A}(\mathcal{O}(r))$ by the von Neumann bicommutant theorem. This operator satisfies

$$
P_{E} A P_{E}=P_{E}\left(a^{*}\left(\mathcal{L}_{r}^{+} e\right)+a\left(\mathcal{L}_{r}^{+} e\right)\right) P_{E} .
$$

In fact, let $\Psi_{1}, \Psi_{2} \in P_{E} \mathcal{H}$. Then, making use of the fact that $\Psi_{1}, \Psi_{2}$ belong to $D_{F}$ in a massive theory, we obtain

$$
\begin{aligned}
\left(\Psi_{1} \mid A \Psi_{2}\right) & =\int d u e^{-\frac{1}{2} u^{2}\left\|\mathcal{L}_{r}^{+} e\right\|^{2}}\left(\Psi_{1} \mid e^{i u\left(a^{*}\left(\mathcal{L}_{r}^{+} e\right)\right.} e^{i u\left(a\left(\mathcal{L}_{r}^{+} e\right)\right)} \Psi_{2}\right) h(u) \\
& =\int d u e^{-\frac{1}{2} u^{2}\left\|\mathcal{L}_{r}^{+} e\right\|^{2}} \sum_{n_{1}, n_{2} \in \mathbb{N}_{0}} \frac{(i u)^{n_{1}+n_{2}}}{n_{1} ! n_{2} !}\left(\Psi_{1} \mid a^{*}\left(\mathcal{L}_{r}^{+} e\right)^{n_{1}} a\left(\mathcal{L}_{r}^{+} e\right)^{n_{2}} \Psi_{2}\right) h(u) \\
& =\left(\Psi_{1} \mid\left(a^{*}\left(\mathcal{L}_{r}^{+} e\right)+a\left(\mathcal{L}_{r}^{+} e\right)\right) \Psi_{2}\right)
\end{aligned}
$$

where in the first step we proceeded to the normal ordered form of $A$, in the second step we expanded the resulting exponentials into the (finite) power series and in the last step we made use of relation (D.3.3). Finally, we pick a function $f \in S(\mathbb{R})$ s.t. $\tilde{f}\left(p_{0}\right)=(2 \pi)^{-\frac{1}{2}}$ for $p^{0} \in[-m,-E]$ and $\tilde{f}\left(p^{0}\right)=0$ for $p^{0} \notin\left[-\frac{1}{2} m,-2 E\right]$. By smearing both sides of equality (D.3.7) in time with $f$ we obtain $A(f) P_{E}=a\left(\mathcal{L}_{r}^{+} e\right) P_{E}$ where we made use of the fact that $A(f)$ is energy-decreasing. Since it is also almost local, the proof is complete.

Thus we have verified relation (D.3.2). It remains to establish suitable convergence properties of expansion (D.1.2). For this purpose we need the following lemma which is similar to Lemma D.2.2 above.

Lemma D.3.2. Let $(\bar{\mu}, \bar{\nu})$ be a pair of 2-multiindices s.t. $|\bar{\mu}| \cdot|\bar{\nu}| \neq 0$. Then there holds in massive scalar free field theory for any $s \geq 1$

$$
\left\|S_{\bar{\mu}, \bar{\nu}}\right\|_{E, 1} \leq c_{r, E} E^{\frac{|\bar{\mu}|+|\bar{\nu}|}{2}} t^{\bar{\mu}+\bar{\nu}}
$$

where the constant $c_{r, E}$ is independent of $\bar{\mu}, \bar{\nu}$.

Proof. For any compact subset $K \subset \mathbb{R}^{s}$ we obtain from the Cauchy-Schwarz inequality

$$
\begin{aligned}
& \sup _{\varphi \in \mathcal{T}_{E, 1}^{+}} \int_{K} d^{s} x\left|\varphi\left(S_{\bar{\mu}, \bar{\nu}}(\vec{x})\right)\right| \\
\leq & \sup _{\varphi \in \mathcal{T}_{E, 1}^{+}}\left(\int_{K} d^{s} x\left|\varphi\left(S_{\bar{\mu}, \bar{\mu}}(\vec{x})\right)\right|\right)^{\frac{1}{2}} \sup _{\varphi \in \mathcal{T}_{E, 1}^{+}}\left(\int_{K} d^{s} x\left|\varphi\left(S_{\bar{\nu}, \bar{\nu}}(\vec{x})\right)\right|\right)^{\frac{1}{2}} .
\end{aligned}
$$

We decompose $\bar{\mu}$ into two 2-multiindices $\bar{\mu}=\bar{\mu}_{a}+\bar{\mu}_{b}$ in such a way that $\left|\bar{\mu}_{b}\right|=1$.

$$
\begin{array}{r}
\int_{K} d^{s} x\left|\varphi\left(S_{\bar{\mu}, \bar{\mu}}(\vec{x})\right)\right|=\int_{K} d^{s} x\left|\varphi\left(\left(a^{*}\left(\mathcal{L}_{r} e\right)^{\bar{\mu}_{b}} P_{E} a^{*}\left(\mathcal{L}_{r} e\right)^{\bar{\mu}_{a}} a\left(\mathcal{L}_{r} e\right)^{\bar{\mu}_{a}} P_{E} a\left(\mathcal{L}_{r} e\right)^{\bar{\mu}_{b}}\right)(\vec{x})\right)\right| \\
\leq\left\|a\left(\mathcal{L}_{r} e\right)^{\bar{\mu}_{a}} P_{E}\right\|^{2} \int_{K} d^{s} x \varphi\left(\left(a^{*}\left(\mathcal{L}_{r} e\right)^{\bar{\mu}_{b}} a\left(\mathcal{L}_{r} e\right)^{\bar{\mu}_{b}}\right)(\vec{x})\right) \\
\leq E^{|\bar{\mu}|} t^{2 \bar{\mu}_{a}} \sup _{\omega(\vec{p}) \leq E}\left|\tilde{h}_{r}(\vec{p})\right|^{-2} \int_{\Delta K} d^{s} x\left|\left\langle\omega^{\frac{1}{2}} \tilde{h}_{r}\left(\mathcal{L}_{r} e\right)^{\bar{\mu}_{b}} \mid U(\vec{x}) \omega^{\frac{1}{2}} \tilde{h}_{r}\left(\mathcal{L}_{r} e\right)^{\bar{\mu}_{b}}\right\rangle\right| \\
\leq c_{r, E}^{2} E^{|\bar{\mu}|} t^{2 \bar{\mu}} .(\mathrm{D} .
\end{array}
$$


Here in the third step we applied the bound (D.1.6) and Lemma C.3.1 with $h=\tilde{h}_{r}^{-1}$, where $\tilde{h}_{r}$ entered into definition (B.4.28) of the operator $T$. In the last step we made use of Lemma C.3.7 which guarantees the convergence of the integral in the massive case. The second factor on the r.h.s. of relation (D.3.10) satisfies an analogous bound. Thus we obtain

$$
\sup _{\varphi \in \mathcal{T}_{E, 1}^{+},} \int_{K} d^{s} x\left|\varphi\left(\stackrel{\vee}{S}_{\bar{\mu}, \bar{\nu}}(\vec{x})\right)\right| \leq c_{r, E}^{2} E^{\frac{|\overline{\mid}|+|\bar{\nu}|}{2}} t^{\bar{\mu}+\bar{\nu}} .
$$

Since the r.h.s. is independent of $K$, we can take the limit $K \nearrow \mathbb{R}^{s}$. Now the statement of the lemma follows from the bound (D.2.4 $)$ after readjusting the constant $c_{r, E}$.

The last auxiliary result which we need to establish Condition $L_{\sharp}^{(1)}$ is the following summability property.

Lemma D.3.3. Let $g \in S(\mathbb{R})$ be s.t. supp $\widetilde{g} \subset]-m, m[$. Then, for arbitrary dimension of space $s \geq 1$, in massive scalar free field theory of mass $m$ there holds the bound

$$
\sum_{\substack{\bar{\mu}, \bar{\nu} \\|\bar{\mu}|+|\bar{\nu}| \neq 0}}\left\|\tau_{\bar{\mu}, \bar{\nu}}\right\|\left\|S_{\bar{\mu}, \bar{\nu}}(g)\right\|_{E, 1}<\infty .
$$

Proof. Making use of Lemma D.2.1, we get

$$
\begin{aligned}
\sum_{\substack{\bar{\mu}, \bar{\nu} \\
|\bar{\mu}|+|\bar{\nu}| \neq 0}}\left\|\tau_{\bar{\mu}, \bar{\nu}}\right\|\left\|S_{\bar{\mu}, \bar{\nu}}(g)\right\|_{E, 1} & =\sum_{\substack{\bar{\mu}, \bar{\nu} \\
|\bar{\mu}| \cdot|\bar{\nu}| \neq 0}}\left\|\tau_{\bar{\mu}, \bar{\nu}}\right\|\left\|S_{\bar{\mu}, \bar{\nu}}(g)\right\|_{E, 1} \\
& \leq 2^{5 M_{E}} c_{r, E}\|g\|_{1} \sum_{\substack{\bar{\mu}, \bar{\nu} \\
|\bar{\mu}| \cdot|\bar{\nu}| \neq 0}} E^{\frac{|\bar{\mu}|+|\bar{\nu}|}{2}} t^{\bar{\mu}+\bar{\nu}} \\
& \leq 2^{5 M_{E}} c_{r, E}\|g\|_{1}\left(\sum_{\substack{\mu^{+} \\
\left|\mu^{+}\right| \leq M_{E}}} E^{\frac{\left|\mu^{+}\right|}{2}} t^{\mu^{+}}\right)^{4} .
\end{aligned}
$$

Here in the second step we made use of Lemma D.3.2 estimate (D.1.8) and the bound (D.2.10). The last expression is finite due to the following relation

$$
\sum_{\substack{\mu^{+} \\\left|\mu^{+}\right| \leq M_{E}}} E^{\frac{\left|\mu^{+}\right|}{2}} t^{\mu^{+}}=\sum_{k=0}^{M_{E}} E^{\frac{k}{2}} \sum_{\substack{\mu^{+} \\\left|\mu^{+}\right|=k}} t^{\mu^{+}} \leq \sum_{k=0}^{M_{E}} E^{\frac{k}{2}}\|T\|_{1}^{k},
$$

where in the last step we made use of the fact that the multinomial coefficients are larger or equal to one and of the multinomial formula (B.1.4).

According to Lemma D.3.3, for any $\varepsilon>0$ there exists a finite set $\mathcal{M}_{\mathrm{f}}$ of pairs of 2multiindices s.t.

$$
\left\|A(g)-\sum_{(\bar{\mu}, \bar{\nu}) \in \mathcal{M}_{\mathrm{f}}} \tau_{\bar{\mu}, \bar{\nu}}(A) S_{\bar{\mu}, \bar{\nu}}(g)\right\|_{E, 1} \leq \varepsilon .
$$

In view of relation (D.3.2), proved in Lemma D.3.1, there exists $C \in \mathfrak{C}$ s.t.

$$
\sum_{(\bar{\mu}, \bar{\nu}) \in \mathcal{M}_{\mathrm{f}}} \tau_{\bar{\mu}, \bar{\nu}}(A) S_{\bar{\mu}, \bar{\nu}}(g)=P_{E} C(g) P_{E} .
$$


122 Appendix D. Verification of Condition $L_{\sharp}^{(1)}$ in Massive Scalar Free Field Theory

Substituting this formula to relation (D.3.16) we establish property (D.3.1). We summarize:

Theorem D.3.4. Condition $L_{\sharp}^{(1)}$ holds in massive scalar free field theory for any dimension of space $s \geq 1$. 


\section{Appendix E}

\section{Verification of Condition $N_{\natural}$ in Scalar Free Field Theory}

It is the goal of the present appendix to verify that Condition $N_{\text {G }}$, stated in Section 3.5, holds both in massive and massless scalar free field theory. The argument relies on Lemma E.2.1 stated below, which is a variant of Theorem 1.6.1 adopted to the problem at hand. This result is combined with Lemma C.3.7 which describes the decay of correlations between certain operators under translations in space.

The necessary background material from Appendix B is summarized in Section E.1. The verification argument, based on the publication Dy08.2 of the author, is given in Section E.2.

\section{E.1 Preliminaries}

The main object of our interest is the map $\Pi_{E}^{\mathrm{c}}: \mathcal{T}_{E} \rightarrow \mathfrak{A}_{\mathrm{c}}(\mathcal{O}(r))^{*}$, given by

$$
\Pi_{E}^{\mathrm{c}}(\varphi)=\left.\varphi\right|_{\mathfrak{A}_{\mathrm{c}}(\mathcal{O}(r))}, \quad \varphi \in \mathcal{T}_{E}
$$

for some $E \geq 0, r>0$. In order to find a suitable expansion of this map into rank-one mappings, we note that there holds

$$
\Pi_{E}^{\mathrm{c}}(\varphi)(A)=\varphi\left(\Theta_{E}(A)\right), \quad A \in \mathfrak{A}_{\mathrm{c}}(\mathcal{O}(r)) .
$$

The map $\Theta_{E}: \mathfrak{A}(\mathcal{O}(r)) \rightarrow B(\mathcal{H})$, given by $\Theta_{E}(A)=P_{E} A P_{E}$, was thoroughly studied in the literature [BP90, Bos00, and we summarized the relevant results in Appendix B] In particular, we recall from Proposition B.5.5 the following decomposition into rank-one maps

$$
\Theta_{E}(A)=\sum_{\bar{\mu}, \bar{\nu}} \tau_{\bar{\mu}, \bar{\nu}}(A) S_{\bar{\mu}, \bar{\nu}}, \quad A \in \mathfrak{A}(\mathcal{O}(r)),
$$

where the sum extends over all pairs of 2-multiindices and converges in the norm topology of $B(\mathcal{H})$. According to definition (B.5.35) and estimate (B.5.36), the bounded operators $S_{\bar{\mu}, \bar{\nu}}$ are given by

$$
S_{\bar{\mu}, \bar{\nu}}=P_{E} a^{*}\left(\mathcal{L}_{r} e\right)^{\bar{\mu}} a\left(\mathcal{L}_{r} e\right)^{\bar{\nu}} P_{E}
$$

and satisfy the bound

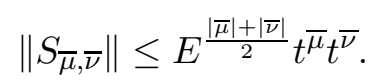


Here $\left\{e_{j}\right\}_{1}^{\infty}$ are the eigenvectors of the operator $T$, defined by (B.4.28), and $\left\{t_{j}\right\}_{1}^{\infty}$ are the corresponding eigenvalues. The normal functionals $\tau_{\bar{\mu}, \bar{\nu}}$ can be estimated as follows

$$
\left\|\tau_{\bar{\mu}, \bar{\nu}}\right\| \leq \frac{2^{\frac{5}{2}(|\bar{\mu}|+|\bar{\nu}|)}}{(\bar{\mu} ! \bar{\nu} !)^{\frac{1}{2}}} .
$$

(See relation $(\underline{B .5 .34})$ ). In view of equality $(\underline{E .1 .2})$ we obtain from (E.1.3) the following expansion for any $\varphi \in \mathcal{T}_{E}, A \in \mathfrak{A}_{\mathrm{c}}(\mathcal{O}(r))$

$$
\Pi_{E}^{\mathrm{c}}(\varphi)(A)=\sum_{\substack{\bar{\mu}, \bar{\nu} \\(|\bar{\mu}|,|\bar{\nu}|) \neq(0,0)}} \tau_{\bar{\mu}, \bar{\nu}}(A) \varphi\left(S_{\bar{\mu}, \bar{\nu}}\right),
$$

where we made use of the fact that $\tau_{0,0}=\omega_{0}$. We associate with operators $S_{\bar{\mu}, \bar{\nu}}$ elements of $\mathcal{T}_{E}^{*}$, denoted by the same symbol, given by $\mathcal{T}_{E} \ni \varphi \rightarrow \varphi\left(S_{\bar{\mu}, \bar{\nu}}\right)$. Their norms are clearly equal to the operator norms of $S_{\bar{\mu}, \bar{\nu}}$. Thus we obtain from Proposition B.5.5 and definition of the $p$-norms stated in Section 3.5

$$
\left\|\Pi_{E}^{\mathrm{c}}\right\|_{p}^{p} \leq \sum_{\substack{\overline{\bar{\mu}, \bar{\nu}} \\(|\bar{\mu}|,|\bar{\nu}|) \neq(0,0)}}\left\|\tau_{\bar{\mu}, \bar{\nu}}\right\|^{p}\left\|S_{\bar{\mu}, \bar{\nu}}\right\|^{p}<\infty .
$$

That is, the maps $\Pi_{E}^{\mathrm{c}}$ are $p$-nuclear w.r.t. the standard norms on $\mathcal{L}\left(\mathcal{T}_{E}, \mathfrak{A}_{\mathrm{c}}(\mathcal{O}(r))\right)$. We will use the same expansion (E.1.7) to estimate the $p$-norms of these maps w.r.t. the norms $\|\cdot\|_{x_{1}, \ldots, x_{N}}$, given by (3.5.3). There clearly holds the bound

$$
\left\|\Pi_{E}^{\mathrm{c}}\right\|_{p, x_{1}, \ldots, x_{N}} \leq\left(\sum_{\substack{\bar{\mu}, \bar{\nu} \\(|\bar{\mu}|,|\bar{\nu}|) \neq(0,0)}}\left\|\tau_{\bar{\mu}, \bar{\nu}}\right\|^{p}\left\|S_{\bar{\mu}, \bar{\nu}}\right\|_{x_{1}, \ldots, x_{N}}^{p}\right)^{\frac{1}{p}} .
$$

To verify Condition $N_{\natural}$, we have to find estimates on the norms $\left\|S_{\bar{\mu}, \bar{\nu}}\right\|_{x_{1}, \ldots, x_{N}}$, whose growth with $N$ can be controlled at large spacelike distances $x_{i}-x_{j}$ for $i \neq j$. We undertake this task in the next section.

\section{E.2 Verification of Condition $N_{\natural}$}

The crucial ingredient of the argument is the following lemma which is inspired by Lemma 2.2 from Bu90]. (See also Theorem 1.6.1). Similarly as in Lemma C.3.1 the present estimate is uniform in the particle number and depends only on the energy of the state in question. This result substantiates the underlying physical idea of additivity of energy over isolated subregions.

Lemma E.2.1. Let $E \geq 0$ and $h$ be a Borel function on $\mathbb{R}^{s}$ which is bounded on $\{\vec{p} \in$ $\left.\mathbb{R}^{s} \mid \omega(\vec{p}) \leq E\right\}$. We denote the operator of multiplication by $h$ on $L^{2}\left(\mathbb{R}^{s}, d^{s} p\right)$ by the same symbol. Suppose that $g \in L^{2}\left(\mathbb{R}^{s}, d^{s} p\right)$ is in the domain of $h \omega^{\frac{1}{2}}$. Then, for any $x_{1}, \ldots, x_{N} \in \mathbb{R}^{s+1}$, there holds the bound

$$
\begin{aligned}
& \left\|P_{E} \sum_{k=1}^{N}\left(a^{*}\left(h \omega^{\frac{1}{2}} g\right) a\left(h \omega^{\frac{1}{2}} g\right)\right)\left(x_{k}\right) P_{E}\right\| \\
& \quad \leq E \sup _{\omega(\vec{p}) \leq E}|h(\vec{p})|^{2}\left\{\|g\|_{2}^{2}+(N-1) \sup _{i \neq j}\left|\left\langle g \mid U\left(x_{i}-x_{j}\right) g\right\rangle\right|\right\} .
\end{aligned}
$$


Proof. We pick single-particle vectors $\Psi_{1}, g_{1} \in L^{2}\left(\mathbb{R}^{s}, d^{s} p\right)$ and define $Q=$ $\sum_{k=1}^{N}\left(a^{*}\left(g_{1}\right) a\left(g_{1}\right)\right)\left(x_{k}\right)$. Then there holds

$$
\begin{aligned}
\left(\Psi_{1} \mid Q Q \Psi_{1}\right) & \leq \sum_{l=1}^{N}\left(\Psi_{1} \mid\left(a^{*}\left(g_{1}\right) a\left(g_{1}\right)\right)\left(x_{l}\right) \Psi_{1}\right) \sum_{k=1}^{N}\left|\left\langle U\left(x_{k}\right) g_{1} \mid U\left(x_{l}\right) g_{1}\right\rangle\right| \\
& \leq\left(\Psi_{1} \mid Q \Psi_{1}\right)\left\{\left\|g_{1}\right\|_{2}^{2}+(N-1) \sup _{i \neq j}\left|\left\langle U\left(x_{j}\right) g_{1} \mid U\left(x_{i}\right) g_{1}\right\rangle\right|\right\},
\end{aligned}
$$

where we made use of the fact that $a\left(U\left(x_{k}\right) g_{1}\right) a\left(U\left(x_{l}\right) g_{1}\right) \Psi_{1}=0$ and of the CauchySchwarz inequality. Since $\left(\Psi_{1} \mid Q \Psi_{1}\right)^{2} \leq\left(\Psi_{1} \mid Q Q \Psi_{1}\right)\left\|\Psi_{1}\right\|^{2}$, we obtain

$$
\begin{aligned}
\sum_{k=1}^{N}\left(\Psi_{1} \mid\right. & \left.\left(a^{*}\left(g_{1}\right) a\left(g_{1}\right)\right)\left(x_{k}\right) \Psi_{1}\right) \\
& \leq\left\|\Psi_{1}\right\|^{2}\left\{\left\|g_{1}\right\|_{2}^{2}+(N-1) \sup _{i \neq j}\left|\left\langle U\left(x_{j}\right) g_{1} \mid U\left(x_{i}\right) g_{1}\right\rangle\right|\right\} .
\end{aligned}
$$

Next, let $n \geq 1$ and $\Psi_{n} \in P_{E} \mathcal{H}$ be an $n$-particle vector s.t. the corresponding symmetric wavefunction $\Psi_{n}\left(\vec{p}_{1}, \ldots, \vec{p}_{n}\right)$ belongs to $S\left(\mathbb{R}^{s \times n}\right)$. We also introduce a single-particle wavefunction associated with $\Psi_{n}$ given by $\Psi_{1}\left(\vec{p}_{1}\right)_{\vec{p}_{2}, \ldots, \vec{p}_{n}}=\omega\left(\vec{p}_{1}\right)^{\frac{1}{2}} \vec{h}\left(\vec{p}_{1}\right) \Psi_{n}\left(\vec{p}_{1}, \ldots, \vec{p}_{n}\right)$, where we treat $\vec{p}_{2}, \ldots, \vec{p}_{n}$ as parameters. With the help of relation (E.2.3) we get

$$
\begin{aligned}
& \sum_{k=1}^{N}\left(\Psi_{n} \mid\left(a^{*}\left(h \omega^{\frac{1}{2}} g\right) a\left(h \omega^{\frac{1}{2}} g\right)\right)\left(x_{k}\right) \Psi_{n}\right) \\
& \quad=n \int d^{s} p_{2} \ldots d^{s} p_{n} \sum_{k=1}^{N}\left(\Psi_{1, \vec{p}_{2}, \ldots, \vec{p}_{n}} \mid\left(a^{*}(g) a(g)\right)\left(x_{k}\right) \Psi_{1, \vec{p}_{2}, \ldots, \vec{p}_{n}}\right) \\
& \leq n \int d^{s} p_{1} \ldots d^{s} p_{n}\left|h\left(\vec{p}_{1}\right)\right|^{2} \omega\left(\vec{p}_{1}\right)\left|\Psi_{n}\left(p_{1}, \ldots, p_{n}\right)\right|^{2} \\
& \cdot\left\{\|g\|_{2}^{2}+(N-1) \sup _{i \neq j}\left|\left\langle g \mid U\left(x_{i}-x_{j}\right) g\right\rangle\right|\right\}
\end{aligned}
$$

Finally, we note that

$$
\begin{aligned}
n \int & d^{s} p_{1} \ldots d^{s} p_{n}\left|h\left(\vec{p}_{1}\right)\right|^{2} \omega\left(\vec{p}_{1}\right)\left|\Psi_{n}\left(\vec{p}_{1}, \ldots, \vec{p}_{n}\right)\right|^{2} \\
\quad \leq \sup _{\omega(\vec{p}) \leq E}|h(\vec{p})|^{2} \int d^{s} p_{1} \ldots d^{s} p_{n}\left(\omega\left(\vec{p}_{1}\right)+\cdots+\omega\left(\vec{p}_{n}\right)\right)\left|\Psi_{n}\left(\vec{p}_{1}, \ldots, \vec{p}_{n}\right)\right|^{2} & \quad \leq \sup _{\omega(\vec{p}) \leq E}|h(\vec{p})|^{2} E\left\|\Psi_{n}\right\|^{2}
\end{aligned}
$$

where we made use of the fact that the wavefunction is symmetric. Since the operators $\left(a^{*}(g) a(g)\right)\left(x_{k}\right)$ conserve the particle number and vectors of the form $\Psi=c_{0} \Omega+\sum_{n=1}^{\infty} \Psi_{n}$, where $\|\Psi\|^{2}=\left|c_{0}\right|^{2}+\sum_{n=1}^{\infty}\left\|\Psi_{n}\right\|^{2}<\infty$, are dense in $P_{E} \mathcal{H}$, we easily obtain the bound in the statement of the lemma.

Our next task is to control the expressions appearing on the r.h.s. of estimate (E.2.1). Similarly as in Subsection C.3.3 we recall from Lemma B.4.5 that $T_{h, \pm}=\omega^{-\gamma} \tilde{h}_{r}^{1 / 2} \mathcal{L}_{r}^{ \pm}$are bounded operators for any $\frac{1}{2} \leq \gamma<\frac{s-1}{2}$ and $\tilde{h}_{r}>0$. Thus we can set in Lemma E.2.1 
$h=\tilde{h}_{r}^{-1}$ and $g=\omega^{-\frac{1}{2}} \tilde{h}_{r} \mathcal{L}_{r}^{ \pm} e$, where $e$ is an eigenvector of the operator $T$ given by (B.4.28). The resulting estimate, studied in Proposition E.2.4 below, relies on the decay properties of the functions

$$
\mathbb{R}^{s+1} \ni x \rightarrow\left\langle\omega^{-\frac{1}{2}} \tilde{h}_{r} \mathcal{L}_{r}^{ \pm} e \mid U(x) \omega^{-\frac{1}{2}} \tilde{h}_{r} \mathcal{L}_{r}^{ \pm} e\right\rangle
$$

which appear on the right-hand side of relation (E.2.1). We studied these functions for $x=(0, \vec{x})$ in Lemma C.3.7 In order to obtain estimates which are valid for arbitrary spacelike translations $x$, we recall, in a slightly generalized form, the following result from BDL87.

Lemma E.2.2. Let $\delta>0$. Then there exists some continuous function $f(\omega)$ which decreases almost exponentially, i.e. $\sup _{\omega}|f(\omega)| e^{|\omega|^{\kappa}}<\infty$ for any $0<\kappa<1$, and which has the property that for any pair of operators $A, B$ s.t. $\Omega$ belongs to their domains and to the domains of their adjoints, satisfying

$$
\left(\Omega \mid\left[A, e^{i t H} B e^{-i t H}\right] \Omega\right)=0 \text { for }|t|<\delta,
$$

there holds the identity $(\Omega \mid A B \Omega)=\frac{1}{2}\{(\Omega \mid A f(\delta H) B \Omega)+(\Omega \mid B f(\delta H) A \Omega)\}$.

With the help of the above lemma we prove the desired bounds.

Lemma E.2.3. Assume that $s \geq 3$. Let $e \in L^{2}\left(\mathbb{R}^{s}, d^{s} p\right)$ satisfy $T e=t e, J e=e$ and $\|e\|=1$. Then, for any $\varepsilon>0,0<\kappa<1$ and $x \in \mathbb{R}^{s+1}$ s.t. $|\vec{x}| \geq\left|x^{0}\right|$, there hold the estimates

$$
\left|\left\langle\tilde{h}_{r} \omega^{-\frac{1}{2}} \mathcal{L}_{r}^{ \pm} e \mid U(x) \tilde{h}_{r} \omega^{-\frac{1}{2}} \mathcal{L}_{r}^{ \pm} e\right\rangle\right| \leq \frac{c_{r, \varepsilon, \kappa}(m) e^{-\frac{1}{2}\left(\frac{m}{5}\right)^{\kappa}\left(|\vec{x}|-\left|x^{0}\right|\right)^{\kappa}} t^{2}}{\left(|\vec{x}|-\left|x^{0}\right|+1\right)^{s-2-\varepsilon}}
$$

where the constant $c_{r, \varepsilon, \kappa}(m) \geq 1$ is independent of $x$ and $e$, and finite for any $m \geq 0$. (If $m>0$, the bound holds for $s \geq 1$ ).

Proof. First, we define the operators $\phi^{+}(e)=\frac{1}{\sqrt{2}}\left(a^{*}\left(\tilde{h}_{r} \mathcal{L}_{r}^{+} e\right)+a\left(\tilde{h}_{r} \mathcal{L}_{r}^{+} e\right)\right), \phi^{-}(e)=$ $\frac{1}{\sqrt{2}}\left(a^{*}\left(i \tilde{h}_{r} \mathcal{L}_{r}^{-} e\right)+a\left(i \tilde{h}_{r} \mathcal{L}_{r}^{-} e\right)\right)$ and their translates $\phi^{ \pm}(e)(x)=U(x) \phi^{ \pm}(e) U(x)^{-1}$. Since the projections $\mathcal{L}_{r}^{ \pm}$and the multiplication operators $\tilde{h}_{r}$ commute with $J$, and $J e=e$, the operators $\phi^{ \pm}(e)$ are localized in the double cone of radius $2 r$ centered at zero. In fact, by definition of the projections $\mathcal{L}_{r}^{ \pm}$, we can find, for any $\varepsilon^{\prime}>0$, functions $g_{ \pm} \in D\left(\mathcal{O}_{r}\right)_{\mathbb{R}}$ s.t. $\left\|\mathcal{L}_{r}^{ \pm} e-\omega^{ \pm \frac{1}{2}} \widetilde{g}_{ \pm}\right\|_{2} \leq \varepsilon^{\prime}$. Setting $F^{ \pm}=(2 \pi)^{-\frac{s}{2}} h_{r} * g_{ \pm}$we obtain from definitions (B.2.4) (B.2.5) of the canonical fields and momenta the following bound

$$
\left\|\phi^{ \pm}(e) \Omega-\phi_{ \pm}\left(F^{ \pm}\right) \Omega\right\|=\frac{1}{\sqrt{2}}\left\|\tilde{h}_{r} \mathcal{L}_{r}^{ \pm} e-\tilde{h}_{r} \omega^{ \pm \frac{1}{2}} \widetilde{g}_{ \pm}\right\|_{2} \leq \frac{\left\|\tilde{h}_{r}\right\|_{\infty} \varepsilon^{\prime}}{\sqrt{2}}
$$

Thus for $x \in \mathbb{R}^{s+1}$ s.t. $\mathcal{O}_{2 r}+x$ and $\mathcal{O}_{2 r}$ are spacelike separated and for any combination of \pm signs there holds

$$
\left[\phi^{ \pm}(e), \phi^{ \pm}(e)(x)\right]=\left(\Omega \mid\left[\phi^{ \pm}(e), \phi^{ \pm}(e)(x)\right] \Omega\right)=0 .
$$

Now we are ready to prove the desired estimate: We assume without loss of generality that $x^{0}>0$, introduce functions $G^{ \pm}(\tau)=\left\langle\tilde{h}_{r} \mathcal{L}_{r}^{ \pm} e \mid \omega^{-1} U\left(\vec{x}+\tau \hat{e}_{0}\right) \tilde{h}_{r} \mathcal{L}_{r}^{ \pm} e\right\rangle$ for $0 \leq \tau \leq x^{0}$, where $\hat{e}_{0}$ is the unit vector in the time direction, and consider the derivative

$$
\left|\frac{d G^{ \pm}(\tau)}{d \tau}\right|=2\left|\left(\Omega \mid \phi^{ \pm}(e) \phi^{ \pm}(e)\left(\vec{x}+\tau \hat{e}_{0}\right) \Omega\right)\right|
$$


We define $\delta_{\tau}=|\vec{x}|-\tau-4 r$ and assume that $|\vec{x}|-x^{0} \geq 5 r$ what guarantees that $\delta_{\tau}>0$ for $0 \leq \tau \leq x^{0}$. Then, by relation (E.2.10), $\phi^{ \pm}(e)$ and $\phi^{ \pm}(e)\left(\vec{x}+\tau \hat{e}_{0}\right)$ satisfy the assumptions of Lemma E.2.2 with $\delta=\delta_{\tau}$. Making use of this result, we obtain

$$
\begin{aligned}
\left|\frac{d G^{ \pm}(\tau)}{d \tau}\right| & =\frac{1}{2} \mid\left\langle\omega^{-\gamma} \tilde{h}_{r} \mathcal{L}_{r}^{ \pm} e \mid \omega^{2 \gamma} f\left(\delta_{\tau} \omega\right) e^{\frac{1}{2}\left(\delta_{\tau} \omega\right)^{\kappa}} e^{-\frac{1}{2}\left(\delta_{\tau} \omega\right)^{\kappa}} U\left(\vec{x}+\tau \hat{e}_{0}\right) \omega^{-\gamma} \tilde{h}_{r} \mathcal{L}_{r}^{ \pm} e\right\rangle \\
& +\left\langle\omega^{-\gamma} \tilde{h}_{r} \mathcal{L}_{r}^{ \pm} e \mid \omega^{2 \gamma} f\left(\delta_{\tau} \omega\right) e^{\frac{1}{2}\left(\delta_{\tau} \omega\right)^{\kappa}} e^{-\frac{1}{2}\left(\delta_{\tau} \omega\right)^{\kappa}} U\left(-\vec{x}-\tau \hat{e}_{0}\right) \omega^{-\gamma} \tilde{h}_{r} \mathcal{L}_{r}^{ \pm} e\right\rangle \mid \\
& \leq \frac{1}{\delta_{\tau}^{2 \gamma}} t^{2}\left\|\tilde{h}_{r}\right\|_{\infty} \sup _{\omega \geq 0}\left|\omega^{2 \gamma} e^{\frac{1}{2} \omega^{\kappa}} f(\omega)\right| e^{-\frac{1}{2} m^{\kappa}\left(|\vec{x}|-x^{0}-4 r\right)^{\kappa}} .
\end{aligned}
$$

Next, we set $\gamma=\frac{s-1-\varepsilon}{2}$ for $0<\varepsilon<1$ and arrive at the following estimate

$$
\begin{aligned}
\left|\left\langle\omega^{-\frac{1}{2}} \tilde{h}_{r} \mathcal{L}_{r}^{ \pm} e \mid U(x) \omega^{-\frac{1}{2}} \tilde{h}_{r} \mathcal{L}_{r}^{ \pm} e\right\rangle\right|=\left|G^{ \pm}\left(x^{0}\right)\right| & \leq\left|G^{ \pm}(0)\right|+\int_{0}^{x^{0}} d \tau\left|\frac{d G^{ \pm}(\tau)}{d \tau}\right| \\
& \leq \frac{c_{r, \varepsilon, \kappa}(m) e^{-\frac{1}{2}\left(\frac{m}{5}\right)^{\kappa}\left(|\vec{x}|-x^{0}\right)^{\kappa}} t^{2}}{\left(|\vec{x}|-x^{0}+1\right)^{s-2-\varepsilon}}
\end{aligned}
$$

where in the last step we applied Lemma C.3.7 and estimate (E.2.12). Since the 1.h.s. of relation (E.2.13) satisfies a uniform bound analogous to (C.3.34), we obtain the estimate in the statement of the lemma.

Now we are ready to prove the required estimates on the norms of the functionals $S_{\bar{\mu}, \bar{\nu}}$.

Proposition E.2.4. Given a family of points $x_{1}, \ldots, x_{N} \in \mathbb{R}^{s+1}$, we define $\delta(\underline{\vec{x}})=$ $\inf _{i \neq j}\left(\left|\vec{x}_{i}-\vec{x}_{j}\right|-\left|x_{i}^{0}-x_{j}^{0}\right|\right)$. For $s \geq 3, \delta(\underline{\vec{x}}) \geq 0$, $(|\bar{\mu}|,|\bar{\nu}|) \neq(0,0)$ and any $\varepsilon>0$, $0<\kappa<1$ the functionals $S_{\bar{\mu}, \bar{\nu}}$ satisfy the bound

$\left\|S_{\bar{\mu}, \bar{\nu}}\right\|_{x_{1}, \ldots, x_{N}}^{2} \leq 16 c_{r, \varepsilon, \kappa}(m) \sup _{\omega(\vec{p}) \leq E}\left|\tilde{h}_{r}(\vec{p})\right|^{-2} E^{|\bar{\mu}|+|\bar{\nu}|} t^{2(\bar{\mu}+\bar{\nu})}\left\{1+(N-1) \frac{e^{-\frac{1}{2}\left(\frac{m}{5}\right)^{\kappa} \delta(\underline{\vec{x}})^{\kappa}}}{(\delta(\underline{\vec{x}})+1)^{s-2-\varepsilon}}\right\}$,

where the constant $c_{r, \varepsilon, \kappa}(m) \geq 1$ appeared in Lemma E.2.3 and is finite for any $m \geq 0$. (If $m>0$, the bound holds for $s \geq 1$ ).

Proof. We denote by $\mathcal{T}_{E, 1}^{+}$the set of positive functionals from $\mathcal{T}_{E, 1}$. Making use of the definition of $\|\cdot\|_{x_{1}, \ldots, x_{N}}$, decomposition (1.6.2) and the Cauchy-Schwarz inequality, we obtain

$$
\begin{aligned}
\left\|S_{\bar{\mu}, \bar{\nu}}\right\|_{x_{1}, \ldots, x_{N}}^{2}=\sup _{\varphi \in \mathcal{T}_{E, 1}} \sum_{k=1}^{N}\left|\varphi_{x_{k}}\left(S_{\bar{\mu}, \bar{\nu}}\right)\right|^{2} \leq 16 \sup _{\varphi \in \mathcal{T}_{E, 1}^{+},} \sum_{k=1}^{N}\left|\varphi_{x_{k}}\left(a^{*}\left(\mathcal{L}_{r} e\right)^{\bar{\mu}} a\left(\mathcal{L}_{r} e\right)^{\bar{\nu}}\right)\right|^{2} \\
\quad \leq 16 \sup _{\varphi \in \mathcal{T}_{E, 1}^{+}} \sum_{k=1}^{N} \varphi_{x_{k}}\left(a^{*}\left(\mathcal{L}_{r} e\right)^{\bar{\mu}} a\left(\mathcal{L}_{r} e\right)^{\bar{\mu}}\right) \varphi_{x_{k}}\left(a^{*}\left(\mathcal{L}_{r} e\right)^{\bar{\nu}} a\left(\mathcal{L}_{r} e\right)^{\bar{\nu}}\right) \\
\leq 16 E^{|\bar{\mu}|} t^{2 \bar{\mu}}\left\|P_{E} \sum_{k=1}^{N}\left(a^{*}\left(\mathcal{L}_{r} e\right)^{\bar{\nu}} a\left(\mathcal{L}_{r} e\right)^{\bar{\nu}}\right)\left(x_{k}\right) P_{E}\right\|,
\end{aligned}
$$

where in the last step we applied the bound (E.1.5). We can assume, without loss of generality, that $\bar{\nu} \neq 0$ and decompose it into two 2-multiindices $\bar{\nu}=\bar{\nu}_{a}+\bar{\nu}_{b}$ in such a way 
that $\left|\bar{\nu}_{b}\right|=1$. Since $a\left(\mathcal{L}_{r} e\right)^{\bar{\nu}}=a\left(\mathcal{L}_{r} e\right)^{\bar{\nu}_{a}} a\left(\mathcal{L}_{r} e\right)^{\bar{\nu}_{b}}$, we get

$$
\begin{aligned}
P_{E} \sum_{k=1}^{N}\left(a^{*}\left(\mathcal{L}_{r} e\right)^{\bar{\nu}} a\left(\mathcal{L}_{r} e\right)^{\bar{\nu}}\right)\left(x_{k}\right) P_{E} \\
\quad=P_{E} \sum_{k=1}^{N}\left(a^{*}\left(\mathcal{L}_{r} e\right)^{\bar{\nu}_{b}} P_{E} a^{*}\left(\mathcal{L}_{r} e\right)^{\bar{\nu}_{a}} a\left(\mathcal{L}_{r} e\right)^{\bar{\nu}_{a}} P_{E} a\left(\mathcal{L}_{r} e\right)^{\bar{\nu}_{b}}\right)\left(x_{k}\right) P_{E} \\
\leq\left\|a\left(\mathcal{L}_{r} e\right)^{\bar{\nu}_{a}} P_{E}\right\|^{2} P_{E} \sum_{k=1}^{N}\left(a^{*}\left(\mathcal{L}_{r} e\right)^{\bar{\nu}_{b}} a\left(\mathcal{L}_{r} e\right)^{\bar{\nu}_{b}}\right)\left(x_{k}\right) P_{E} \\
=E^{\left|\bar{\nu}_{a}\right|} t^{2 \bar{\nu}_{a}} P_{E} \sum_{k=1}^{N}\left(a^{*}\left(\mathcal{L}_{r} e\right)^{\bar{\nu}_{b}} a\left(\mathcal{L}_{r} e\right)^{\bar{\nu}_{b}}\right)\left(x_{k}\right) P_{E}
\end{aligned}
$$

where in the last step we used again estimate (E.1.5). From relations (E.2.15) and (E.2.16) we obtain the bound

$$
\left\|S_{\bar{\mu}, \bar{\nu}}\right\|_{x_{1}, \ldots, x_{N}}^{2} \leq 16 E^{|\bar{\mu}|+\left|\bar{\nu}_{a}\right|} t^{2\left(\bar{\mu}+\bar{\nu}_{a}\right)}\left\|P_{E} \sum_{k=1}^{N}\left(a^{*}\left(\mathcal{L}_{r} e\right)^{\bar{\nu}_{b}} a\left(\mathcal{L}_{r} e\right)^{\bar{\nu}_{b}}\right)\left(x_{k}\right) P_{E}\right\|
$$

From Lemmas E.2.1 and E.2.3 we get

$$
\begin{aligned}
\| P_{E} \sum_{k=1}^{N}\left(a^{*}\right. & \left.\left(\mathcal{L}_{r} e\right)^{\bar{\nu}_{b}} a\left(\mathcal{L}_{r} e\right)^{\bar{\nu}_{b}}\right)\left(x_{k}\right) P_{E} \| \leq E \sup _{\omega(\vec{p}) \leq E}\left|\tilde{h}_{r}(\vec{p})\right|^{-2}\left\{\left\|\tilde{h}_{r} \omega^{-\frac{1}{2}}\left(\mathcal{L}_{r} e\right)^{\bar{\nu}_{b}}\right\|^{2}\right. \\
& \left.+(N-1) \sup _{i \neq j}\left|\left\langle\tilde{h}_{r} \omega^{-\frac{1}{2}}\left(\mathcal{L}_{r} e\right)^{\bar{\nu}_{b}} \mid U\left(x_{i}-x_{j}\right) \tilde{h}_{r} \omega^{-\frac{1}{2}}\left(\mathcal{L}_{r} e\right)^{\bar{\nu}_{b}}\right\rangle\right|\right\} \\
& \leq c_{r, \varepsilon, \kappa}(m) \sup _{\omega(\vec{p}) \leq E}\left|\tilde{h}_{r}(\vec{p})\right|^{-2} E t^{2 \bar{\nu}_{b}}\left\{1+(N-1) \frac{e^{-\frac{1}{2}\left(\frac{m}{5}\right)^{\kappa} \delta(\underline{\vec{x}})^{\kappa}}}{(\delta(\underline{\vec{x}})+1)^{s-2-\varepsilon}}\right\} .(\mathrm{E}
\end{aligned}
$$

Substituting inequality (E.2.18) to formula (E.2.17), we obtain the estimate in the statement of the proposition.

We note that the bound from Proposition E.2.4 has similar structure to estimate (E.1.5) for the ordinary norms of $S_{\bar{\mu}, \bar{\nu}}$. Therefore, making use of formulas (E.1.9) and (E.1.6), and proceeding as in the proof of Lemma B.5.5, we obtain

$$
\begin{aligned}
\left\|\Pi_{E}^{\mathrm{c}}\right\|_{p, x_{1}, \ldots, x_{N}} \leq 4\left(c_{r, \varepsilon, \kappa}(m)\right)^{1 / 2} \sup _{\omega(\vec{p}) \leq E}|\tilde{h}(\vec{p})|^{-1}\left(\sum_{k=0}^{\infty} \frac{\left(2^{5} E\right)^{\frac{1}{2} p k}\left\|T^{p}\right\|_{1}^{k}}{(k !)^{\frac{1}{2} p}}\right)^{\frac{4}{p}} \\
\cdot\left\{1+(N-1) \frac{e^{-\frac{1}{2}\left(\frac{m}{5}\right)^{\kappa} \delta(\underline{\vec{x}})^{\kappa}}}{(\delta(\underline{\vec{x}})+1)^{s-2-\varepsilon}}\right\}^{\frac{1}{2}} .
\end{aligned}
$$

It follows that $\lim \sup _{\delta(\underline{\vec{x}}) \rightarrow \infty}\left\|\Pi_{E}^{\mathrm{c}}\right\|_{p, x_{1}, \ldots, x_{N}}$ satisfies a bound which is independent of $N$. Consequently, we get

Theorem E.2.5. Condition $N_{\natural}$ holds in scalar free field theory in $s \geq 3$ dimensional space. (If $m>0$, the same is true for $s \geq 1$ ). 
It is obvious that Condition $N_{\natural}$ holds also in the sub-theory $\left(\mathfrak{A}^{(\mathrm{d})}, \alpha, \mathcal{H}\right)$, generated by the derivatives of the field, since all our bounds remain valid if one restricts attention to a smaller set of observables. It also holds in the even part of scalar free field theory $\left(\underline{\mathfrak{A}}^{(\mathrm{e})}, \underline{\alpha}, \mathcal{H}^{(\mathrm{e})}\right)$ as can be seen by the following argument: We consider the map $\underline{\Pi}_{E}^{\mathrm{c}}: \mathcal{T}_{E}^{(\mathrm{e})} \rightarrow$ $\underline{\mathfrak{A}}_{\mathrm{c}}^{(\mathrm{e})}(\mathcal{O})^{*}$ given by

$$
\underline{\Pi}_{E}^{\mathrm{c}}(\underline{\varphi})=\left.\underline{\varphi}\right|_{\underline{\mathfrak{A}}_{\mathrm{c}}^{(\mathrm{e})}(\mathcal{O})}, \quad \underline{\varphi} \in \mathcal{T}_{E}^{(\mathrm{e})} .
$$

Due to formula (B.2.18), we obtain

$$
\underline{\Pi}_{E}^{\mathrm{c}}(\underline{\varphi})(\underline{A})=\Pi_{E}^{\mathrm{c}}(\iota(\mathrm{e}) \underline{\varphi})\left(\pi_{(\mathrm{e})}^{-1}(\underline{A})\right)
$$

for any $\underline{\varphi} \in \mathcal{T}_{E}^{(\mathrm{e})}$ and $\underline{A} \in \underline{\mathfrak{A}}_{\mathrm{c}}^{(\mathrm{e})}(\mathcal{O})$. Thus the expansion of the map $\Pi_{E}^{\mathrm{c}}$ into rank-one mappings, given by (E.1.7), induces the corresponding expansion of the map $\underline{\Pi}_{E}^{\mathrm{c}}$

$$
\underline{\Pi}_{E}^{\mathrm{c}}(\underline{\varphi})(\underline{A})=\sum_{\substack{\bar{\mu}, \bar{\nu} \\(|\bar{\mu}|,|\bar{\nu}|) \neq(0,0)}} \underline{\tau}_{\bar{\mu}, \bar{\nu}}(\underline{A}) \underline{S}_{\bar{\mu}, \bar{\nu}}(\underline{\varphi}),
$$

where $\underline{\tau}_{\bar{\mu}, \bar{\nu}}(\underline{A})=\tau_{\bar{\mu}, \bar{\nu}}\left(\pi_{(\mathrm{e})}^{-1}(\underline{A})\right)$ and $\underline{S}_{\bar{\mu}, \bar{\nu}}(\underline{\varphi})=S_{\bar{\mu}, \bar{\nu}}\left(\iota_{(\mathrm{e})} \underline{\varphi}\right)$. Making use of the facts that $\|\underline{A}\|=\left\|\pi_{(\mathrm{e})}^{-1}(\underline{A})\right\|$ and $\|\underline{\varphi}\|=\left\|\iota_{(\mathrm{e})} \underline{\varphi}\right\|$, justified in Section $\mathbb{B} .2$, we obtain that $\left\|\underline{\tau}_{\bar{\mu}, \bar{\nu}}\right\| \leq$ $\left\|\tau_{\bar{\mu}, \bar{\nu}}\right\|$ and $\left\|\underline{S}_{\bar{\mu}, \bar{\nu}}\right\|_{x_{1}, \ldots, x_{N}} \leq\left\|S_{\bar{\mu}, \bar{\nu}}\right\|_{x_{1}, \ldots, x_{N}}$. It follows that the $p$-norms $\left\|\underline{\Pi}_{E}^{\mathrm{c}}\right\|_{p, x_{1}, \ldots, x_{N}}$ satisfy the bound (E.2.19). Thus we obtain:

Corollary E.2.6. Let $s \geq 3$. Then Condition $N_{\bullet}$ holds in the even part of scalar free field theory $\left(\underline{\mathfrak{A}}^{(e)}, \underline{\alpha}, \mathcal{H}^{(e)}\right)$ and in the sub-theory $\left(\mathfrak{A}^{(d)}, \alpha, \mathcal{H}\right)$ generated by the derivatives of the field. (If $m>0$, the same is true for $s \geq 1$ ). 


\section{Appendix F}

\section{Verification of Condition $C_{\mathrm{b}}$ in Massive Scalar Free Field Theory}

We showed in Theorem 3.3.1 that the qualitative part (a) of Condition $C_{\mathrm{b}}$ holds in all theories satisfying Condition $C_{\sharp}$, in particular in (massive and massless) scalar free field theory in physical spacetime BP90. Moreover, we argued in Section 3.3 that in physically meaningful, massive theories there should also hold the strengthened, quantitative part (b) of this condition. We demonstrated that this quantitative refinement has a number of interesting consequences pertaining to the vacuum structure. It is the goal of the present appendix to illustrate the mechanism which enforces Condition $C_{\mathrm{b}}$ (b) by a direct computation in the theory of massive, non-interacting particles.

This appendix is organized as follows: In Section [F.1 we present the argument relying on some technical information stated in Lemma F.1.3 below. The remaining two sections are devoted to the proof of this lemma.

\section{F.1 Main Line of Argument}

In Section 3.2 we introduced, for any $\underline{\vec{x}} \in \Gamma_{N, \delta}$ and $\varphi \in \mathcal{T}_{E}$, the following elements of $\left(\mathfrak{A}(\mathcal{O}(r))^{\times N}\right)^{*}$

$$
\varphi_{\underline{\vec{x}}}\left(A_{1} \times \cdots \times A_{N}\right)=\varphi\left(A_{1}\left(\vec{x}_{1}\right) \ldots A_{N}\left(\vec{x}_{N}\right)\right)
$$

and considered the maps $\Pi_{E, N, \delta} \in \mathcal{L}\left(\mathcal{T}_{E} \times \Gamma_{N, \delta},\left(\mathfrak{A}(\mathcal{O}(r))^{\times N}\right)^{*}\right)$ given by

$$
\Pi_{E, N, \delta}(\varphi, \underline{\vec{x}})=\varphi_{\underline{\vec{x}}} .
$$

In Section 3.3 we introduced the maps $\Pi_{E, N, \delta}^{\mathrm{c}} \in \mathcal{L}\left(\mathcal{T}_{E} \times \Gamma_{N, \delta},\left(\mathfrak{A}_{\mathrm{c}}(\mathcal{O}(r))^{\times N}\right)^{*}\right)$ defined as

$$
\Pi_{E, N, \delta}^{\mathrm{c}}(\varphi, \underline{\vec{x}})=\left.\Pi_{E, N, \delta}(\varphi, \underline{\vec{x}})\right|_{\mathfrak{A}_{\mathrm{c}}(\mathcal{O}(r))^{\times N}} .
$$

It is our goal to show that $\lim _{\delta \rightarrow 0}\left\|\Pi_{E, N, \delta}^{\mathrm{c}}\right\|=0$. Then, by Lemma 3.3.2 there follows the statement of Condition $C_{\mathrm{b}}(\mathrm{b})$.

Theorem F.1.1. Massive scalar free field theory satisfies Condition $C_{\mathrm{b}}$ for any dimension of space $s \geq 1$.

Proof. The main ingredient of the proof consists in the following elementary evaluation of the $N$-linear form $\Pi_{E, N, \delta}^{\mathrm{c}}(\varphi, \underline{\vec{x}})$, where $\varphi \in \mathcal{T}_{E, 1}, \underline{\vec{x}} \in \Gamma_{N, \delta}$, on the generating elements 
of $\mathfrak{A}_{\mathrm{c}}(\mathcal{O}(r))$. We pick $f_{1}, \ldots, f_{N} \in \mathcal{L}_{r}$, abbreviate their translates $U(\vec{x}) f_{j}$ as $f_{j, \vec{x}}$ and compute

$$
\begin{aligned}
& \Pi_{E, N, \delta}^{\mathrm{c}}(\varphi, \underline{\vec{x}})\left(\left\{W\left(f_{1}\right)-\omega_{0}\left(W\left(f_{1}\right)\right) I\right\} \times \cdots \times\left\{W\left(f_{N}\right)-\omega_{0}\left(W\left(f_{N}\right)\right) I\right\}\right) \\
& =\varphi\left(\left(W\left(f_{1, \vec{x}_{1}}\right)-\omega_{0}\left(W\left(f_{1}\right)\right) I\right) \ldots\left(W\left(f_{N, \vec{x}_{N}}\right)-\omega_{0}\left(W\left(f_{N}\right)\right) I\right)\right) \\
& =\sum_{R_{1}, R_{2}}(-1)^{\left|R_{2}\right|} e^{-\frac{1}{2} \sum_{k=1}^{\left|R_{2}\right|}\left\|f_{j_{k}}\right\|_{2}^{2}} \varphi\left(W\left(f_{i_{1}, \vec{x}_{i_{1}}}+\cdots+f_{i_{\left|R_{1}\right|}, \vec{x}_{i\left|R_{1}\right|}}\right)\right) \\
& =\sum_{R_{1}, R_{2}}(-1)^{\left|R_{2}\right|} e^{-\frac{1}{2} \sum_{k=1}^{N}\left\|f_{k}\right\|_{2}^{2}} e^{-\sum_{1 \leq k<l \leq\left|R_{1}\right|} \operatorname{Re}\left\langle f_{i_{k}, \vec{x}_{i}} \mid f_{i_{l}, \vec{x}_{i}}\right\rangle} \\
& \cdot \varphi\left(: W\left(f_{i_{1}, \vec{x}_{i_{1}}}\right) \ldots W\left(f_{i_{\left|R_{1}\right|}, \vec{x}_{i R_{1} \mid}}\right):\right) \\
& =\sum_{R_{1}, R_{2}}(-1)^{\left|R_{2}\right|} e^{-\frac{1}{2} \sum_{k=1}^{N}\left\|f_{k}\right\|_{2}^{2}}\left(e^{-\sum_{1 \leq k<l \leq\left|R_{1}\right|} \operatorname{Re}\left\langle f_{i_{k}, \vec{x}_{i}} \mid f_{i_{l}, \vec{x}_{i}}\right\rangle}-1\right) \\
& \cdot \varphi\left(: W\left(f_{i_{1}, \vec{x}_{i_{1}}}\right) \ldots W\left(f_{i_{\left|R_{1}\right|}, \vec{x}_{\left|R_{1}\right|}}\right):\right) \\
& +e^{-\frac{1}{2} \sum_{k=1}^{N}\left\|f_{k}\right\|_{2}^{2}} \sum_{R_{1}, R_{2}}(-1)^{\left|R_{2}\right|} \varphi\left(: W\left(f_{i_{1}, \vec{x}_{i_{1}}}+\cdots+f_{i_{\left|R_{1}\right|}, \vec{x}_{i\left|R_{1}\right|}}\right):\right),
\end{aligned}
$$

where the sum extends over all partitions $R_{1}=\left(i_{1}, \ldots, i_{\left|R_{1}\right|}\right), R_{2}=\left(j_{1}, \ldots, j_{\left|R_{2}\right|}\right)$ of an $N$ element set into two, possibly improper, ordered subsets. (If the condition $1 \leq k<l \leq\left|R_{1}\right|$ is empty, the corresponding sum is understood to be zero). In the second step we made use of the fact that the Weyl operators are localized in spacelike separated regions. In the third step we applied the identity $W(f)=e^{-\frac{1}{2}\|f\|_{2}^{2}}: W(f)$ : and in the last step we divided the expression into two parts: The first part tends to zero for large spacelike separations, due to the decay of $\left\langle f_{1, x_{1}} \mid f_{2, x_{2}}\right\rangle$ when $x_{1}-x_{2}$ tends to spacelike infinity. In the next lemma we show that the last sum on the r.h.s. of (F.1.4) vanishes for $N>2 \frac{E}{m}$, so we can omit this last term in the subsequent discussion.

Lemma F.1.2. Let $\varphi \in \mathcal{T}_{E}$ and $N>2 \frac{E}{m}$ be a natural number. Then there holds

$$
S:=\sum_{R_{1}, R_{2}}(-1)^{\left|R_{2}\right|} \varphi\left(: W\left(f_{i_{1}, \vec{x}_{i_{1}}}+\cdots+f_{i_{\left|R_{1}\right|}, \vec{x}_{\left|R_{1}\right|} \mid}\right):\right)=0,
$$

where the sum extends over all partitions of an $N$-element set.

Proof. For any $f \in L^{2}\left(\mathbb{R}^{s}, d^{s} p\right)$ we introduce the map $M(f): B(\mathcal{H}) \rightarrow B(\mathcal{H})$ given by

$$
M(f)(C)=P_{E} e^{i a^{*}(f)} C e^{i a(f)} P_{E}, \quad C \in B(\mathcal{H}) .
$$

The exponentials are defined by their Taylor expansions which are finite (in the massive theory) due to the energy projections. The range of $M(f)$ belongs to $B(\mathcal{H})$ due to the energy bounds (B.5.22). We note that $M\left(f_{1}\right) M\left(f_{2}\right)=M\left(f_{2}\right) M\left(f_{1}\right)$ for any $f_{1}, f_{2} \in$ $L^{2}\left(\mathbb{R}^{s}, d^{s} p\right)$ and that $M(0)(C)=P_{E} C P_{E}$ for any $C \in B(\mathcal{H})$. We denote by $\hat{I}$ the identity operator acting from $B(\mathcal{H})$ to $B(\mathcal{H})$. There clearly holds

$$
\begin{aligned}
S & =\sum_{R_{1}, R_{2}}(-1)^{\left|R_{2}\right|} \varphi\left(M\left(f_{i_{1}, \vec{x}_{i_{1}}}\right) \ldots M\left(f_{i_{\left|R_{1}\right|}, \vec{x}_{i_{\mid} R_{1} \mid}}\right)(I)\right) \\
& =\varphi\left(\left(M\left(f_{1, \vec{x}_{1}}\right)-\hat{I}\right) \ldots\left(M\left(f_{N, \vec{x}_{N}}\right)-\hat{I}\right)(I)\right) \\
& =\varphi\left(\left(M\left(f_{1, \vec{x}_{1}}\right)-M(0)\right) \ldots\left(M\left(f_{N, \vec{x}_{N}}\right)-M(0)\right)(I)\right),
\end{aligned}
$$


where the last equality holds due to the fact that $\varphi \in \mathcal{T}_{E}$. Finally, we note that for any $C \in B(\mathcal{H})$

$$
(M(f)-M(0))(C)=\sum_{k+l \geq 1} P_{E} \frac{\left(i a^{*}(f)\right)^{k}}{k !} C \frac{(i a(f))^{l}}{l !} P_{E} .
$$

Substituting this relation to (F.1.7), we verify the claim.

We will exploit formula (F.1.4 $)$ to show that for $N>2 \frac{E}{m}$ the norms of the maps $\Pi_{E, N, \delta}^{\mathrm{c}}$ tend to zero with $\delta \rightarrow \infty$. To this end, we introduce the $*$-algebra $\mathfrak{A}(\mathcal{O}(r))$, generated by finite linear combinations of Weyl operators, and denote by $\left(\mathfrak{A}(\mathcal{O}(r))^{\times M}\right)^{*}$ the space of (not necessarily bounded) $M$-linear forms on $\mathfrak{A}(\mathcal{O}(r))$. We define the maps $\Pi_{E, M, \delta}^{\prime}$ : $\mathcal{T}_{E} \times \Gamma_{M, \delta} \rightarrow\left(\mathfrak{\mathfrak { A }}(\mathcal{O}(r))^{\times M}\right)^{*}$, linear in the first argument, extending by linearity the following expression

$$
\begin{aligned}
& \Pi_{E, M, \delta}^{\prime}(\varphi, \underline{\vec{x}})\left(W\left(f_{1}\right) \times \cdots \times W\left(f_{M}\right)\right) \\
& =e^{-\frac{1}{2} \sum_{k=1}^{M}\left\|f_{k}\right\|_{2}^{2}}\left(e^{-\sum_{1 \leq i<j \leq M} \operatorname{Re}\left\langle f_{i, \vec{x}_{i}} \mid f_{j, \vec{x}_{j}}\right\rangle}-1\right) \varphi\left(: W\left(f_{1, \vec{x}_{1}}+\cdots+f_{M, \vec{x}_{M}}\right):\right) .
\end{aligned}
$$

We obtain from (F.1.4) the equality valid for $N>2 \frac{E}{m}$

$$
\begin{aligned}
& \Pi_{E, N, \delta}^{\mathrm{c}}(\varphi, \underline{\vec{x}})\left(\left\{W\left(f_{1}\right)-\omega_{0}(\right.\right.\left.\left.\left.W\left(f_{1}\right)\right) I\right\} \times \cdots \times\left\{W\left(f_{N}\right)-\omega_{0}\left(W\left(f_{N}\right)\right) I\right\}\right) \\
&= \sum_{R_{1}, R_{2}}(-1)^{\left|R_{2}\right|} \omega_{0}\left(W\left(f_{j_{1}}\right)\right) \ldots \omega_{0}\left(W\left(f_{j_{\left|R_{2}\right|}}\right)\right) . \\
& \cdot \Pi_{E,\left|R_{1}\right|, \delta}^{\prime}\left(\varphi, \underline{\vec{x}}_{R_{1}}\right)\left(W\left(f_{i_{1}}\right) \times \cdots \times W\left(f_{i_{\mid R_{1}} \mid}\right)\right),
\end{aligned}
$$

where $\vec{x}_{R_{1}}:=\left(\vec{x}_{i_{1}}, \ldots, \vec{x}_{i_{\left|R_{1}\right|}}\right)$ is clearly an element of $\Gamma_{\left|R_{1}\right|, \delta}$. To conclude the argument, we need the following technical lemma.

Lemma F.1.3. For any $M \in \mathbb{N}, E \geq 0$, double cone $\mathcal{O}(r)$ and sufficiently large $\delta>0$ (depending on $M, E$ and $\mathcal{O}(r)$ ) there exist maps $\Pi_{E, M, \delta}^{\prime \prime} \in \mathcal{L}\left(\mathcal{T}_{E} \times \Gamma_{M, \delta},\left(\mathfrak{A}(\mathcal{O}(r))^{\otimes M}\right)^{*}\right.$ ) which have the properties

(a) $\lim _{\delta \rightarrow \infty}\left\|\Pi_{E, M, \delta}^{\prime \prime}\right\|=0$,

(b) $\Pi_{E, M, \delta}^{\prime \prime}(\varphi, \underline{\vec{x}})\left(A_{1} \otimes \cdots \otimes A_{M}\right)=\Pi_{E, M, \delta}^{\prime}(\varphi, \underline{\vec{x}})\left(A_{1} \times \cdots \times A_{M}\right)$ for $A_{1}, \ldots, A_{M} \in \mathfrak{A}(\mathcal{O}(r))$ and any $(\varphi, \underline{\vec{x}}) \in \mathcal{T}_{E} \times \Gamma_{M, \delta}$.

In view of this lemma, whose proof is postponed to Section F.2, equality (F.1.10) can now be rewritten as follows, for sufficiently large $N, \delta$ and any $A_{1}, \ldots, A_{N} \in \mathfrak{A}_{\mathrm{c}}(\mathcal{O}(r))$

$$
\Pi_{E, N, \delta}^{\mathrm{c}}(\varphi, \underline{\vec{x}})\left(A_{1} \times \cdots \times A_{N}\right)=\Pi_{E, N, \delta}^{\prime \prime}(\varphi, \underline{\vec{x}})\left(A_{1} \otimes \cdots \otimes A_{N}\right),
$$

where we made use of the facts that $\omega_{0}\left(A_{1}\right)=\cdots=\omega_{0}\left(A_{N}\right)=0$ and that $\mathfrak{A}(\mathcal{O}(r))$ is dense in $\mathfrak{A}(\mathcal{O}(r))$ in the strong operator topology. Consequently, for $N>2 \frac{E}{m}$, the maps $\Pi_{E, N, \delta}^{\mathrm{c}}$ share the properties of $\Pi_{E, N, \delta}^{\prime \prime}$ stated in Lemma F.1.3. In addition, we know from Theorem 3.3.1 that the maps $\Pi_{E, N, \delta}^{\mathrm{c}}$ are compact for any $\delta>0$. We conclude that Condition $C_{\mathrm{b}}$ is satisfied.

Now it easily follows that Condition $C_{\mathrm{b}}$ is also satisfied in the even part of massive scalar free field theory $\left(\underline{\mathfrak{A}}^{(\mathrm{e})}, \underline{\alpha}, \mathcal{H}^{(\mathrm{e})}\right)$, introduced in Section B.2. There we have shown that for 
any $\underline{A} \in \underline{\mathfrak{A}}^{(\mathrm{e})}$ and $\underline{\varphi} \in \mathcal{T}_{E}^{(\mathrm{e})}$ there holds $\|\underline{A}\|=\left\|\pi_{(\mathrm{e})}^{-1}(\underline{A})\right\|$ and $\|\underline{\varphi}\|=\left\|\iota_{(\mathrm{e})} \underline{\varphi}\right\|$. Making use of these equalities and formula (B.2.18), one easily obtains the bound

$$
\left\|\underline{\Pi}_{E, N, \delta}^{\mathrm{c}}\right\| \leq\left\|\Pi_{E, N, \delta}^{\mathrm{c}}\right\|,
$$

where the map on the l.h.s. is defined within $\left(\underline{\mathfrak{A}}^{(\mathrm{e})}, \underline{\alpha}, \mathcal{H}^{(\mathrm{e})}\right)$ and the mapping on the r.h.s. corresponds to the full massive scalar free field theory. Thus there follows the corollary:

Theorem F.1.4. The even part of massive scalar free field theory $\left(\underline{\mathfrak{A}}^{(e)}, \underline{\alpha}, \mathcal{H}^{(e)}\right)$ satisfies Condition $C_{\mathrm{b}}$ for any dimension of space $s \geq 1$.

We remark that the assumption $m>0$ is used only in one (crucial) step in the proof of Theorem [F.1.1 namely to eliminate the last term in relation (F.1.4) and establish equality (F.1.11). The properties of the maps $\Pi_{E, M, \delta}^{\prime}$, stated in Lemma F.1.3, hold in massless free field theory as well. However, we do not expect that the present Condition $C_{b}$ holds in massless theories as it stands. There, due to the existence of states with arbitrary number of particles in $\mathcal{T}_{E}$, the maximal number of localization centers $N_{0}$, visible in an experiment, should depend not only on the energy $E$ of the state, but also on the experimental accuracy $\varepsilon$. We conjecture that an accordingly modified condition has similar physical consequences to the present one and that it holds in massless free field theory.

\section{F.2 Proof of Lemma F.1.3}

The goal of this section is to construct the maps $\Pi_{E, M, \delta}^{\prime \prime} \in \mathcal{L}\left(\mathcal{T}_{E} \times \Gamma_{M, \delta},\left(\mathfrak{A}(\mathcal{O}(r))^{\otimes M}\right)^{*}\right)$ and verify that they have the properties (a) and (b) specified in Lemma F.1.3. We will define these maps as norm-convergent sums of rank-one mappings, i.e.

$$
\Pi_{E, M, \delta}^{\prime \prime}=\sum_{i=1}^{\infty} \tau_{i} S_{i}
$$

where $\tau_{i} \in\left(\mathfrak{A}(\mathcal{O}(r))^{\otimes M}\right)^{*}$ and $S_{i} \in \mathcal{L}\left(\mathcal{T}_{E} \times \Gamma_{M, \delta}, \mathbb{C}\right)$.

In order to construct a suitable family of functionals $\tau_{i}$, we recall the relevant results from Bos00, which we reproduced in Proposition B.3.1 For any 2-multiindex $\bar{\mu}=\left(\mu^{+}, \mu^{-}\right)$and an orthonormal basis $\left\{\stackrel{\circ}{e}_{i}\right\}_{1}^{\infty}$ of $J$-invariant eigenvectors in the singleparticle space $L^{2}\left(\mathbb{R}^{s}, d^{s} p\right)$ there exists a normal functional $\tau_{\bar{\mu}}$ on $B(\mathcal{H})$ s.t. for any $f \in \mathcal{L}_{r}$ there holds

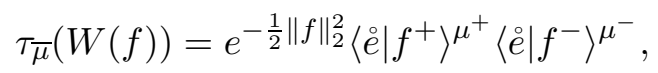

where $f^{+}, f^{-}$are the real and imaginary parts of $f$ in configuration space. These functionals satisfy the bound

$$
\left\|\tau_{\bar{\mu}}\right\| \leq 4^{\left|\mu^{+}\right|+\left|\mu^{-}\right|}\left(\mu^{+} ! \mu^{-} !\right)^{\frac{1}{2}}
$$

Turning to the definition of suitable functionals on $B(\mathcal{H})^{\otimes M}$, we introduce $M$-tuples of multiindices $\underline{\mu}^{ \pm}=\left(\mu^{ \pm}, \ldots, \mu^{ \pm}\right)$and the corresponding $2 M$-multiindices $\underline{\mu}=\left(\underline{\mu}^{+}, \underline{\mu}^{-}\right)$. We 
extend the standard rules of the multiindex notation (see Section B.1) as follows

$$
\begin{aligned}
& |\underline{\mu}|=\sum_{i=1}^{M}\left(\left|\mu_{i}^{+}\right|+\left|\mu_{i}^{-}\right|\right), \\
& \underline{\mu} !=\prod_{i=1}^{M} \mu_{i}^{+} ! \mu_{i}^{-} ! \\
& \langle\grave{e} \mid f\rangle \underline{\mu}=\prod_{i=1}^{M}\left\langle\grave{e} \mid f_{i}^{+}\right\rangle^{\mu_{i}^{+}}\left\langle\grave{e} \mid f_{i}^{-}\right\rangle^{\mu_{i}^{-}},
\end{aligned}
$$

where $f_{1}, \ldots, f_{M} \in \mathcal{L}_{r}$. Now for any $2 M$-multiindex $\underline{\mu}$ we define the normal functional $\tau_{\underline{\mu}}$ on $B(\mathcal{H})^{\otimes M}$ by the expression

$$
\tau_{\underline{\mu}}=\tau_{\bar{\mu}_{1}} \otimes \cdots \otimes \tau_{\bar{\mu}_{M}}
$$

From relations (F.2.2), (F.2.3) and the polar decomposition of a normal functional Sa] one immediately obtains:

Lemma F.2.1. Let $\left\{\stackrel{\circ}{i}_{i}\right\}_{1}^{\infty}$ be an orthonormal basis in $L^{2}\left(\mathbb{R}^{s}\right)$ of $J$-invariant eigenvectors. The functionals $\tau_{\underline{\mu}} \in\left(B(\mathcal{H})^{\otimes M}\right)^{*}$, given by F.2.7), have the following properties

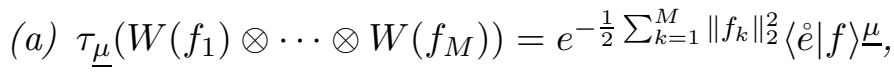

(b) $\left\|\tau_{\underline{\mu}}\right\| \leq 4 \underline{|\mu|}(\underline{\mu} !)^{\frac{1}{2}}$,

where $f_{1}, \ldots, f_{M} \in \mathcal{L}_{r}$.

In order to construct a basis $\left\{\dot{e}_{i}\right\}_{1}^{\infty}$ in $L^{2}\left(\mathbb{R}^{s}, d^{s} p\right)$ of $J$-invariant eigenvectors, which is suitable for our purposes, we modify slightly our discussion from Subsection B.4.2 (based on [BP90, Bos00]). Let $Q_{E}$ be the projection on states of energy lower than $E$ in the single-particle space. We define the operators $\stackrel{\circ}{T}_{E}^{ \pm}=Q_{E} \mathcal{L}_{r}^{ \pm}$and $\stackrel{\circ}{T}_{\kappa}^{ \pm}=e^{-\frac{|\omega|^{\kappa}}{2}} \mathcal{L}_{r}^{ \pm}$, where $0<\kappa<1$. By a slight modification of Lemma B.4.5 one finds that these operators satisfy $\left\|\stackrel{\circ}{T}_{E}^{ \pm}\right\|_{1}<\infty,\left\|\stackrel{\circ}{T}_{\kappa}^{ \pm}\right\|_{1}<\infty$, where $\|\cdot\|_{1}$ denotes the trace norm. Next, we introduce the operator $\stackrel{\circ}{T}$ given by

$$
\stackrel{\circ}{T}=\left(\left|\stackrel{\circ}{T}_{E}^{+}\right|^{2}+\left|\stackrel{\circ}{T}_{E}^{-}\right|^{2}+\left|\stackrel{\circ}{T}_{\kappa}^{+}\right|^{2}+\left|\stackrel{\circ}{T}_{\kappa}^{-}\right|^{2}\right)^{\frac{1}{2}} .
$$

Making use of the estimate $\left\|(A+B)^{p}\right\|_{1} \leq\left\|A^{p}\right\|_{1}+\left\|B^{p}\right\|_{1}$, valid for any $0<p \leq 1$ and any pair of positive operators $A, B$ s.t. $A^{p}, B^{p}$ are trace-class Ko84, we obtain

$$
\|\stackrel{\circ}{T}\|_{1} \leq\left\|\stackrel{\circ}{T}_{E}^{+}\right\|_{1}+\left\|\stackrel{\circ}{T}_{E}^{-}\right\|_{1}+\left\|\stackrel{\circ}{T}_{\kappa}^{+}\right\|_{1}+\left\|\stackrel{\circ}{T}_{\kappa}^{-}\right\|_{1}<\infty .
$$

Since $\stackrel{\circ}{T}$ commutes with $J$, it has a $J$-invariant orthonormal basis of eigenvectors $\left\{\stackrel{\circ}{i}_{i}\right\}_{1}^{\infty}$ and we denote the corresponding eigenvalues by $\left\{\dot{t}_{i}\right\}_{1}^{\infty}$.

Now we proceed to the construction of the functionals $S_{i} \in \mathcal{L}\left(\mathcal{T}_{E} \times \Gamma_{M, \delta}, \mathbb{C}\right)$, to appear in the expansion (F.2.1). Let $\hat{\alpha}^{ \pm}=\left(\alpha_{1,2}^{ \pm}, \ldots, \alpha_{M-1, M}^{ \pm}\right)$be $\left(\begin{array}{c}M \\ 2\end{array}\right)$-tuples of multiindices and let $\hat{\alpha}=\left(\hat{\alpha}^{+}, \hat{\alpha}^{-}\right)$be the corresponding $2\left(\begin{array}{c}M \\ 2\end{array}\right)$-multiindex. First, we define the contribution to the functional which is responsible for the correlations between measurements:

$$
\begin{aligned}
& F_{\hat{\alpha}, \hat{\beta}}(\underline{\vec{x}})=\prod_{1 \leq i<j \leq M} \frac{(-1)^{\left|\alpha_{i, j}^{-}\right|+\left|\alpha_{i, j}^{+}\right|}}{\sqrt{\alpha_{i, j}^{+} ! \beta_{i, j}^{+} ! \alpha_{i, j}^{-} ! \beta_{i, j}^{-} !}}\left(\Omega \mid a\left(\mathcal{L}_{r}^{+}{\stackrel{\circ}{\vec{x}_{i}}}^{\alpha_{i, j}^{+}} a^{*}\left(\mathcal{L}_{r}^{+}{\stackrel{\circ}{\vec{x}_{j}}}_{\vec{x}_{j}}\right)^{\left.\beta_{i, j}^{+} \Omega\right)}\right.\right. \\
& \cdot\left(\Omega \mid a\left(\mathcal{L}_{r}^{-}{\stackrel{\circ}{\vec{x}_{i}}}_{i}\right)^{\alpha_{i, j}^{-}} a^{*}\left(\mathcal{L}_{r}^{-} \stackrel{\circ}{e}_{\vec{x}_{j}}\right)^{\beta_{i, j}^{-}} \Omega\right),
\end{aligned}
$$


where we use the short-hand notation $\mathcal{L}_{r}^{ \pm}{\stackrel{\circ}{i,} \vec{x}_{j}}=U\left(\vec{x}_{j}\right) \mathcal{L}_{r}^{ \pm} \stackrel{\circ}{i}_{i}$. The functionals in question are given by

$$
S_{\underline{\mu}, \underline{\nu}, \hat{\alpha}, \hat{\beta}}(\varphi, \underline{\vec{x}})=\frac{i^{\left|\underline{\mu}^{+}\right|+\left|\underline{\nu}^{+}\right|+2\left|\underline{\mu}^{-}\right|}}{\underline{\mu} ! \underline{\nu} ! \sqrt{\hat{\alpha} ! \hat{\beta} !}} F_{\hat{\alpha}, \hat{\beta}}(\underline{\vec{x}}) \varphi\left(a^{*}\left(\mathcal{L}_{r} \stackrel{\circ}{\vec{x}}^{\prime} \underline{\mu}^{\mu} a\left(\mathcal{L}_{r} \stackrel{\circ}{\vec{x}}_{\vec{x}}\right)^{\underline{\nu}}\right),\right.
$$

where $\varphi \in \mathcal{T}_{E}$ and $\underline{\vec{x}} \in \Gamma_{M, \delta}$. The norms of these functionals satisfy the bound, stated in the following lemma, whose proof is postponed to Section F.3.

Lemma F.2.2. The functionals $S_{\underline{\mu,}, \underline{\hat{\alpha}}, \hat{\beta}} \in \mathcal{L}\left(\mathcal{T}_{E} \times \Gamma_{M, \delta}, \mathbb{C}\right)$, given by (F.2.11), satisfy the following estimates

$$
\left\|S_{\underline{\mu}, \underline{\nu}, \hat{\alpha}, \hat{\beta}}\right\| \leq\left(\frac{M_{E}^{\frac{1}{2}(|\underline{\mu}|+|\underline{\nu}|)}}{\underline{\mu} ! \underline{\nu} !} i^{\underline{\mu}+\underline{\nu}}\right)\left(\frac{1}{\sqrt{\hat{\alpha} ! \hat{\beta} !}} \sqrt{\frac{\left|\hat{\alpha}^{+}\right| !\left|\hat{\alpha}^{-}\right| !\left|\hat{\beta}^{+}\right| !\left|\hat{\beta}^{-}\right| !}{\hat{\alpha} ! \hat{\beta} !}} g(\delta)^{|\hat{\alpha}|+|\hat{\beta}|} t^{\hat{\alpha}+\hat{\beta}}\right),
$$

where $M_{E}=\frac{E}{m},\left\{\dot{t}_{i}\right\}_{1}^{\infty}$ are the eigenvalues of the operator $\stackrel{\circ}{T}$ given by (F.2.8) and the function $g$, which is independent of $\hat{\alpha}$ and $\hat{\beta}$, satisfies $\lim _{\delta \rightarrow \infty} g(\delta)=0$.

Given the estimates from Lemmas F.2.1 (b) and F.2.2, we can proceed to the study of the convergence properties of the expansion (F.2.1). For this purpose we need some notation: For any pair of $\left(\begin{array}{c}M \\ 2\end{array}\right)$-tuples of multiindices $\hat{\alpha}^{ \pm}=\left(\alpha_{1,2}^{ \pm}, \ldots, \alpha_{M-1, M}^{ \pm}\right)$we define the associated $M$-tuples of multiindices $\underset{\hat{\alpha}^{ \pm}}{\rightarrow}, \hat{\alpha}^{ \pm}$as follows

$$
\begin{aligned}
& \hat{\hat{\alpha}}_{i}^{ \pm}=\sum_{\substack{1<j \leq M \\
i<j}} \alpha_{i, j}^{ \pm}, \\
& \stackrel{\hat{\alpha}}{i}_{i}^{ \pm}=\sum_{\substack{1 \leq j<M, j<i}} \alpha_{j, i}^{ \pm},
\end{aligned}
$$

where $i \in\{1, \ldots, M\}$. The corresponding $2 M$-multiindices are denoted by $\stackrel{\hat{\alpha}}{\rightarrow}=\left(\stackrel{\hat{\alpha}^{+}}{\rightarrow}, \hat{\alpha}^{-}\right)$, $\stackrel{\hat{\alpha}}{\leftarrow}=\left(\hat{\alpha}^{+}, \hat{\alpha}^{-}\right)$. The relevant estimate is stated in the following lemma, whose proof is given in Section F.3.

Lemma F.2.3. The functionals $\tau_{\underline{\mu}} \in\left(\mathfrak{A}(\mathcal{O}(r))^{\otimes M}\right)^{*}$ and $S_{\underline{\mu}, \underline{\nu}, \hat{\alpha}, \hat{\beta}} \in \mathcal{L}\left(\mathcal{T}_{E} \times \Gamma_{M, \delta}, \mathbb{C}\right)$ satisfy

$$
\sum_{\substack{\underline{\mu}, \underline{\underline{\nu}} \\ \hat{\alpha}, \hat{\beta} \\ \hat{\hat{\beta}},|\hat{\beta}|) \neq(0,0)}}\left\|\tau_{\underline{\mu}+\underline{\nu}+\underline{\hat{\alpha}} \rightarrow+\hat{\beta}}^{\longrightarrow}\right\|\left\|S_{\underline{\mu}, \underline{\underline{\nu}}, \hat{\alpha}, \hat{\beta}}\right\|<\infty
$$

for sufficiently large $\delta>0$, depending on $M, E$ and the double cone $\mathcal{O}(r)$. Moreover, the above sum tends to zero with $\delta \rightarrow \infty$.

After this preparation we proceed to the main part of this section.

Proof of Lemma F.1.3 We define $\Pi_{E, M, \delta}^{\prime \prime} \in \mathcal{L}\left(\mathcal{T}_{E} \times \Gamma_{M, \delta},\left(\mathfrak{A}(\mathcal{O}(r))^{\otimes M}\right)^{*}\right)$ as follows

$$
\Pi_{E, M, \delta}^{\prime \prime}(\varphi, \underline{\vec{x}})=\sum_{\substack{\underline{\mu}, \underline{\underline{\alpha}}, \hat{\beta} \\(|\hat{\alpha}|,|\hat{\beta}|) \neq(0,0)}} \tau_{\underline{\mu}+\underline{\nu}+\underline{\hat{\alpha}} \rightarrow+\underline{\hat{\beta}}} S_{\underline{\mu,}, \underline{\hat{\alpha}, \hat{\beta}}, \hat{\beta}}(\varphi, \underline{\vec{x}}) .
$$


In view of Lemma F.2.3 this map is well defined for sufficiently large $\delta>0$ and satisfies $\lim _{\delta \rightarrow \infty}\left\|\Pi_{E, M, \delta}^{\prime \prime}\right\|=0$ as required in part (a) of Lemma F.1.3. In order to verify part (b), it suffices to show that

$$
\begin{aligned}
& \Pi_{E, M, \delta}^{\prime \prime}(\varphi, \underline{\vec{x}})\left(W\left(f_{1}\right) \otimes \cdots \otimes W\left(f_{M}\right)\right)=\Pi_{E, M, \delta}^{\prime}(\varphi, \underline{\vec{x}})\left(W\left(f_{1}\right) \times \cdots \times W\left(f_{M}\right)\right) \\
& =e^{-\frac{1}{2} \sum_{k=1}^{M}\left\|f_{k}\right\|_{2}^{2}}\left(e^{-\sum_{1 \leq i<j \leq M}\left(\left\langle f_{i, \vec{x}_{i}}^{+} \mid f_{j, \vec{x}_{j}}^{+}\right\rangle+\left\langle f_{i, \vec{x}_{i}}^{-} \mid f_{j, \vec{x}_{j}}^{-}\right\rangle\right)}-1\right) \\
& \cdot \varphi\left(: W\left(f_{1, \vec{x}_{1}}+\cdots+f_{M, \vec{x}_{M}}\right):\right)
\end{aligned}
$$

where the second equality restates the definition of the map $\Pi_{E, M, \delta}^{\prime}$, given by formula (F.1.9]). The l.h.s. can be evaluated making use of Lemma [F.2.1 (a) and definition (F.2.11)

$$
\begin{aligned}
& \Pi_{E, N, \delta}^{\prime \prime}(\varphi, \underline{\vec{x}})\left(W\left(f_{1}\right) \times \cdots \times W\left(f_{N}\right)\right)
\end{aligned}
$$

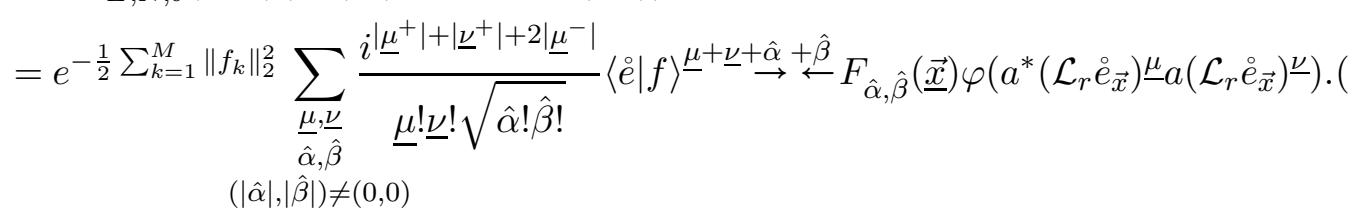

First, we consider the sum w.r.t. $\underline{\mu}, \underline{\nu}$. There holds

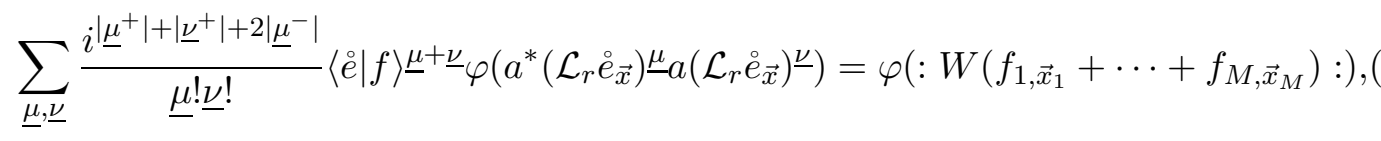

as one can verify by expanding the normal ordered Weyl operator on the r.h.s. into the power series of creation and annihilation operators of the functions $f_{j, \vec{x}_{j}}^{ \pm}$, expanding each function $f_{j}^{ \pm}$in the orthonormal basis $\left\{\stackrel{\circ}{e}_{i}\right\}_{1}^{\infty}$ and making use of the multinomial formula (B.1.4)

$$
a^{(*)}\left(f_{j, \vec{x}_{j}}^{ \pm}\right)^{m_{j}^{ \pm}}=\sum_{\mu_{j}^{ \pm},\left|\mu_{j}^{ \pm}\right|=m_{j}^{ \pm}} \frac{m_{j}^{ \pm} !}{\mu_{j}^{ \pm} !}\left\langle\stackrel{\ominus}{e} \mid f_{j}\right\rangle^{\mu_{j}^{ \pm}} a^{(*)}\left(\mathcal{L}_{r}^{+}{\stackrel{\circ}{\dot{x}_{j}}}_{\vec{x}_{j}}\right)^{\mu_{j}^{ \pm}}
$$

The sum w.r.t. $\hat{\alpha}, \hat{\beta}$ in (F.2.18) gives

$$
\sum_{\substack{\hat{\alpha}, \hat{\beta} \\(|\hat{\alpha}|,|\hat{\beta}|) \neq(0,0)}} \frac{1}{\sqrt{\hat{\alpha} ! \hat{\beta} !}}\langle\grave{e}| f \nmid \stackrel{\hat{\alpha}}{\stackrel{+\hat{\beta}}{\leftarrow}} F_{\hat{\alpha}, \hat{\beta}}(\underline{\vec{x}})=\left(\prod_{1 \leq i<j \leq M} e^{-\left\langle f_{i, \vec{x}_{i}}^{+} \mid f_{j, \vec{x}_{j}}^{+}\right\rangle} e^{-\left\langle f_{i, \vec{x}_{i}}^{-} \mid f_{j, \vec{x}_{j}}^{-}\right\rangle}\right)-1 .
$$

This relation can be verified by expanding the exponential functions on the r.h.s. into the Taylor series, making use of the identity

$$
\left\langle f_{i, \vec{x}_{i}}^{ \pm} \mid f_{j, \vec{x}_{j}}^{ \pm}\right\rangle_{i, j}^{k_{i, j}^{ \pm}}=\frac{\left(\Omega \mid a\left(f_{i, \vec{x}_{i}}^{ \pm}\right)^{k_{i, j}^{+}} a^{*}\left(f_{j, \vec{x}_{j}}^{ \pm}\right)^{\left.k_{i, j}^{+} \Omega\right)}\right.}{k_{i, j}^{+} !}
$$

and applying to the resulting expressions expansions (F.2.20). Comparing (F.2.21) and (F.2.19) with (F.2.17), we conclude the proof of Lemma F.1.3 


\section{F.3 Some Technical Proofs}

In this section we provide proofs of Lemmas F.2.2 and F.2.3 which we used to prove Lemma F.1.3 in the previous section.

The key ingredient of our proof of Lemma F.2.2 is the observation that when the spatial distance between two local operators is large, then the energy transfer between them is heavily damped. We exploited this idea in Section 3.2. where it was encoded in Lemma 3.2.2. In the present context it is more convenient to use a variant of Lemma 2.3 of [BDL87] which is reproduced as Lemma E.2.2 in the present work. With the help of this result we prove the following lemma which will help us to control the correlation terms $F_{\hat{\alpha}, \hat{\beta}}$.

Lemma F.3.1. Let $\delta>0,(\vec{x}, \vec{y}) \in \Gamma_{2, \delta},\left\{\stackrel{\circ}{e}_{i}\right\}_{1}^{\infty}$ be the basis of J-invariant eigenvectors of the operator $\stackrel{\circ}{T}_{\text {given by (F.2.8) }}$, let $\left\{\dot{t}_{i}\right\}_{1}^{\infty}$ be the corresponding eigenvalues and let $\alpha, \beta$ be multiindices. Then there holds, for any combination of \pm signs,

$$
\mid\left(\Omega\left|a\left(\mathcal{L}_{r}^{ \pm} \stackrel{\circ}{\vec{x}}_{\vec{x}}^{\alpha} a^{*}\left(\mathcal{L}_{r}^{ \pm} \stackrel{\circ}{\vec{y}}_{\vec{y}}\right)^{\beta} \Omega\right)\right| \leq \sqrt{|\alpha| !|\beta| !} g(\delta)^{|\alpha|+|\beta|}{ }^{\alpha+\beta},\right.
$$

where $\mathcal{L}_{r}^{ \pm} \stackrel{\circ}{e}_{i, \vec{x}}:=U(\vec{x}) \mathcal{L}_{r}^{ \pm} \stackrel{\circ}{e}_{i}$, the function $g$ is independent of $\alpha, \beta$ and satisfies $\lim _{\delta \rightarrow \infty} g(\delta)=0$.

Proof. We consider here only the $(++)$ case, as the remaining cases are treated analogously. We define the operators $\phi^{+}\left(\stackrel{\circ}{e}_{i}\right)=\frac{1}{\sqrt{2}}\left(a^{*}\left(\mathcal{L}_{r}^{+} \stackrel{\circ}{i}_{i}\right)+a\left(\mathcal{L}_{r}^{+} \stackrel{\circ}{i}_{i}\right)\right)$ and their translates $\phi^{+}\left(\stackrel{\circ}{e}_{i}\right)(\vec{x})=U(\vec{x}) \phi^{+}\left(\stackrel{\circ}{e}_{i}\right) U(\vec{x})^{-1}$. Since the projections $\mathcal{L}_{r}^{ \pm}$commute with $J, J \stackrel{\circ}{i}_{i}=\stackrel{\circ}{i}_{i}$ and $\delta>0$, these operators satisfy the assumptions of Lemma E.2.2 (See the proof of Lemma E.2.3 for a detailed justification). Therefore, we obtain

$$
\begin{aligned}
\left\langle\mathcal{L}_{r}^{+} \stackrel{\circ}{e}_{i, \vec{x}} \mid \mathcal{L}_{r}^{+} \stackrel{\circ}{e}_{j, \vec{y}}\right\rangle=2\left(\Omega \mid \phi^{+}\left(\stackrel{\circ}{e}_{i}\right)(\vec{x}) \phi^{+}\left(\stackrel{\circ}{e}_{j}\right)(\vec{y}) \Omega\right) & =\left(\Omega \mid \phi^{+}\left(\stackrel{\circ}{e}_{i}\right)(\vec{x}) f(\delta H) \phi^{+}\left(\stackrel{\circ}{e}_{j}\right)(\vec{y}) \Omega\right)+\left(\Omega \mid \phi^{+}\left(\stackrel{\circ}{e}_{j}\right)(\vec{y}) f(\delta H) \phi^{+}\left(\stackrel{\circ}{e}_{i}\right)(\vec{x}) \Omega\right) \\
& =\frac{1}{2}\left(\left\langle\mathcal{L}_{r}^{+} \stackrel{\circ}{e}_{i, \vec{x}} \mid f(\delta \omega) \mathcal{L}_{r}^{+} \stackrel{\circ}{e}_{j, \vec{y}}\right\rangle+\left\langle\mathcal{L}_{r}^{+} \stackrel{\circ}{e}_{j, \vec{y}} \mid f(\delta \omega) \mathcal{L}_{r}^{+} \stackrel{\circ}{e}_{i, \vec{x}}\right\rangle\right),
\end{aligned}
$$

where the function $f$ was defined in Lemma E.2.2. Making use of this result, exploiting the fact that the 1.h.s. of $(\mathbb{F . 3 . 1})$ vanishes for $|\alpha| \neq|\beta|$ and setting $|\alpha|=|\beta|=k$, we get

$$
\begin{aligned}
& \left(\Omega \mid a\left(\mathcal{L}_{r}^{+} \stackrel{\circ}{\vec{x}}_{\vec{x}}\right)^{\alpha} a^{*}\left(\mathcal{L}_{r}^{+} \stackrel{\circ}{e}_{\vec{y}}\right)^{\beta} \Omega\right) \\
& =\left(\Omega \mid a\left(\mathcal{L}_{r}^{+} \stackrel{\circ}{e}_{i_{1}, \vec{x}}\right) \ldots a\left(\mathcal{L}_{r}^{+}{\stackrel{\circ}{i_{k}, \vec{x}}}_{i}\right) a^{*}\left(\mathcal{L}_{r}^{+} \stackrel{\circ}{e}_{j_{1}, \vec{y}}\right) \ldots a^{*}\left(\mathcal{L}_{r}^{+}{\stackrel{\circ}{j_{k}, \vec{y}}}_{j}\right) \Omega\right) \\
& =\sum_{\sigma \in S_{k}}\left\langle\mathcal{L}_{r}^{+}{\stackrel{\circ}{e_{1}, \vec{x}}} \mid \mathcal{L}_{r}^{+} \stackrel{\circ}{e}_{j_{\sigma_{1}}, \vec{y}}\right\rangle \ldots\left\langle\mathcal{L}_{r}^{+}{\stackrel{\circ}{e_{k}, \vec{x}}} \mid \mathcal{L}_{r}^{+} \stackrel{\circ}{e}_{j_{\sigma_{k}}, \vec{y}}\right\rangle \\
& =\sum_{\sigma \in S_{k}} \frac{1}{2}\left(\left\langle\mathcal{L}_{r}^{+} \stackrel{\circ}{e}_{i_{1}, \vec{x}} \mid f(\delta \omega) \mathcal{L}_{r}^{+} \stackrel{\circ}{e}_{j_{\sigma_{1}}, \vec{y}}\right\rangle+\left\langle\mathcal{L}_{r}^{+}{\stackrel{\circ}{e_{\sigma_{1}}, \vec{y}}} \mid f(\delta \omega) \mathcal{L}_{r}^{+\stackrel{\circ}{e}_{i_{1}, \vec{x}}}\right\rangle\right) \\
& \ldots \frac{1}{2}\left(\left\langle\mathcal{L}_{r}^{+}{\stackrel{\circ}{e_{k}, \vec{x}}} \mid f(\delta \omega) \mathcal{L}_{r}^{+}{\stackrel{\circ}{j_{\sigma_{k}}, \vec{y}}}\right\rangle+\left\langle\mathcal{L}_{r}^{+}{\stackrel{\circ}{j_{\sigma_{k}}, \vec{y}}}\right| f(\delta \omega) \mathcal{L}_{r}^{+}{\stackrel{\circ}{i_{k}, \vec{x}}}\right),
\end{aligned}
$$

where the sum extends over all permutations of a $k$-element set. For any $0<\kappa<1$ there holds $c_{f}^{2}:=\sup _{\omega}\left|f(\omega) e^{|\omega|^{\kappa}}\right|<\infty$. Consequently, we get

$$
\begin{aligned}
\left|\left\langle\mathcal{L}_{r}^{+} \stackrel{\circ}{e}_{i, \vec{x}} \mid f(\delta \omega) \mathcal{L}_{r}^{+} \stackrel{\circ}{e}_{j, \vec{y}}\right\rangle\right| & =\left|\left\langle\mathcal{L}_{r}^{+} \stackrel{\circ}{e}_{i, \vec{x}} \mid f(\delta \omega) e^{(\delta|\omega|)^{\kappa}} e^{-\left(\delta^{\kappa}-1\right)|\omega|^{\kappa}} e^{-|\omega|^{\kappa}} \mathcal{L}_{r}^{+} \stackrel{\circ}{e}_{j, \vec{y}}\right\rangle\right| \\
& \leq c_{f}^{2} e^{-\left(\delta^{\kappa}-1\right) m^{\kappa}}\left\|e^{-\frac{|\omega|^{\kappa}}{2}} \mathcal{L}_{r}^{+} \stackrel{\circ}{e}_{i}\right\|\left\|e^{-\frac{|\omega|^{\kappa}}{2}} \mathcal{L}_{r}^{+} \stackrel{\circ}{e}_{j}\right\| .
\end{aligned}
$$


Finally, we note that $\left\|e^{-\frac{|\omega|^{\kappa}}{2}} \mathcal{L}_{r}^{+} \stackrel{\circ}{e}_{i}\right\|=\left\|\stackrel{\circ}{T}_{\kappa}^{+} \stackrel{\circ}{e}_{i}\right\| \leq\left\|\stackrel{\circ}{T}_{i}\right\|=\stackrel{\circ}{t}_{i}$ and the claim follows.

After this preparation we proceed to the proof of Lemma F.2.2

Proof of Lemma F.2.2, Exploiting the energy bounds (B.5.22) and the fact that $\left\|\omega^{-\frac{1}{2}}\right\|=m^{-\frac{1}{2}}$ in the massive theory, we obtain

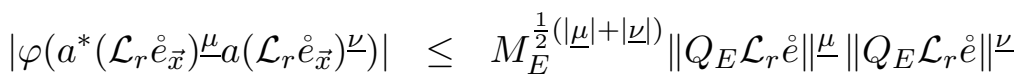

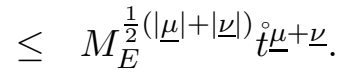

Next, with the help of Lemma F.3.1 we analyze the expressions $F_{\hat{\alpha}, \hat{\beta}}$, given by (F.2.10),

$$
\begin{array}{r}
\left|F_{\hat{\alpha}, \hat{\beta}}(\underline{\vec{x}})\right| \leq \prod_{1 \leq i<j \leq M} \sqrt{\frac{\left|\alpha_{i, j}^{+}\right| !\left|\beta_{i, j}^{+}\right| !\left|\alpha_{i, j}^{-}\right| !\left|\beta_{i, j}^{-}\right| !}{\alpha_{i, j}^{+} ! \beta_{i, j}^{+} ! \alpha_{i, j}^{-} ! \beta_{i, j}^{-} !}}\left(g(\delta) \AA^{\left.\alpha^{\alpha}\right)_{i, j}^{+}+\beta_{i, j}^{+}+\alpha_{i, j}^{-}+\beta_{i, j}^{-}}\right. \\
\leq \sqrt{\frac{\left|\hat{\alpha}^{+}\right| !\left|\hat{\alpha}^{-}\right| !\left|\hat{\beta}^{+}\right| !\left|\hat{\beta}^{-}\right| !}{\hat{\alpha} ! \hat{\beta} !}} g(\delta)^{|\hat{\alpha}|+|\hat{\beta}|} t^{\hat{\alpha}+\hat{\beta}},
\end{array}
$$

where we made use of the estimate $\prod_{1 \leq i<j \leq M}\left|\alpha_{i, j}^{+}\right| ! \leq\left(\sum_{1<i<j \leq M}\left|\alpha_{i, j}^{+}\right|\right) !=\left|\hat{\alpha}^{+}\right|$!. Altogether, combining (F.3.5) and (F.3.6), we obtain from (F.2.11) the bound (F.2.12).

We conclude this appendix with the proof of Lemma F.2.3.

Proof of Lemma F.2.3 First, we estimate the norms of the functionals $\tau_{\underline{\mu}+\underline{\nu}+\underline{\hat{\alpha}} \rightarrow+\underset{\hat{\beta}}{\leftarrow}}$. Making use of the bound stated in Lemma F.2.1 (b), and of the fact that $(a+b+\vec{c}) ! \leq$ $3^{a+b+c} a ! b ! c$ ! for any $a, b, c \in \mathbb{N}_{0}$, which follows from the properties of the multinomial coefficients, we get

$$
\begin{aligned}
\left\|\tau_{\underline{\mu}+\underline{\nu}+\underline{\hat{\alpha}} \rightarrow+\underline{\hat{\beta}}}\right\| & \leq 4^{|\underline{\mu}|+|\underline{\nu}|+|\underline{\hat{\alpha}}|+|\underline{\hat{\beta}}|} \sqrt{(\underline{\mu}+\underline{\nu}+\underline{\hat{\alpha}}+\underline{\hat{\beta}}) !} \\
& \leq\left((4 \sqrt{3})^{|\underline{\mu}|+|\underline{\nu}|} \sqrt{(\underline{\mu}+\underline{\nu}) !}\right)\left((4 \sqrt{3})^{|\hat{\alpha}|+|\hat{\beta}|} \sqrt{\underline{\hat{\alpha}} ! \underline{\hat{\beta}} !}\right),
\end{aligned}
$$

where we noted that $|\underline{\hat{\alpha}}|=|\hat{\alpha}|$ and $\underline{\hat{\beta}}|=| \hat{\beta} \mid$. (See definitions $(\underline{\mathrm{F} .2 .13})$ and $(\underline{\mathbb{F} .2 .14})$ ).

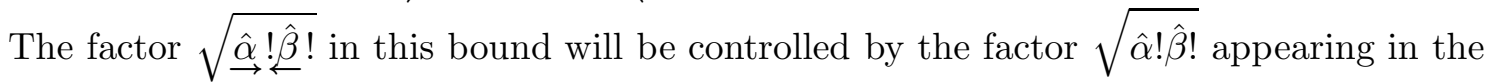
denominator in (F.2.12). We note the relevant estimate

$$
\begin{aligned}
\stackrel{\hat{\alpha} !}{\vec{\alpha} !}=\frac{\hat{\alpha}^{+}}{\overrightarrow{\hat{\alpha}^{+}} !} \stackrel{\hat{\alpha}^{-} !}{\overrightarrow{\hat{\alpha}^{-} !}} & =\prod_{i=1}^{M} \frac{\left(\sum_{1<j \leq M, j>i} \alpha_{i, j}^{+}\right) !}{\left(\prod_{1<j \leq M, j>i} \alpha_{i, j}^{+}\right) !} \frac{\left(\sum_{1<j \leq M, j>i} \alpha_{i, j}^{-}\right) !}{\left(\prod_{1<j \leq M, j>i} \alpha_{i, j}^{-}\right) !} \\
& \leq M^{\sum_{1 \leq i<j \leq M}\left(\left|\alpha_{i, j}^{+}\right|+\left|\alpha_{i, j}^{-}\right|\right)} \leq M^{|\hat{\alpha}|},
\end{aligned}
$$

where we made use of the properties of the multinomial coefficients. Similarly, the factor $\sqrt{(\underline{\mu}+\underline{\nu}) !}$ appearing in (F.3.7) will be counterbalanced by $\sqrt{\underline{\mu} \underline{\underline{\nu}} \underline{!}}$ extracted from the denominator of (F.2.12). The relevant estimate relies on the property of the binomial coefficients

$$
\frac{(\underline{\mu}+\underline{\nu}) !}{\underline{\mu} ! \underline{\underline{\nu}} !} \leq 2 \underline{|\mu|+|\underline{\nu}|}
$$


With the help of the last two bounds and relations (F.2.12), (F.3.7) we obtain

$$
\begin{aligned}
& \sum_{\substack{\underline{\mu}, \underline{\underline{\nu}} \\
\hat{\alpha}, \hat{\beta}}}\left\|\tau_{\underline{\mu}+\underline{\nu}+\underline{\hat{\alpha}} \rightarrow \underline{\hat{\beta}}}\right\|\left\|S_{\underline{\mu}, \underline{,}, \hat{\alpha}, \hat{\beta}}\right\| \leq\left(\sum_{\underline{\mu}, \underline{\nu}} \frac{\left(4 \sqrt{6 M_{E}}\right) \underline{|\underline{\mu}|+|\underline{\nu}|}}{\sqrt{\underline{\mu} ! \underline{\underline{\nu}} !}} t^{\mu}+\underline{\underline{\nu}}\right) \\
& (|\hat{\alpha}|,|\hat{\beta}|) \neq(0,0) \\
& \cdot\left(\sum_{\hat{\alpha}, \hat{\beta}} \sqrt{\frac{\left|\hat{\alpha}^{+}\right| !\left|\hat{\alpha}^{-}\right| !\left|\hat{\beta}^{+}\right| !\left|\hat{\beta}^{-}\right| !}{\hat{\alpha} ! \hat{\beta} !}}(4 \sqrt{3 M} g(\delta))^{|\hat{\alpha}|+|\hat{\beta}|} t^{\hat{\alpha}+\hat{\beta}}\right), \\
& (|\hat{\alpha}|,|\hat{\beta}|) \neq(0,0)
\end{aligned}
$$

where we made use of the bound (F.3.8). The sum w.r.t. $\underline{\mu}, \underline{\nu}$ can be easily estimated as it factorizes into $4 M$ independent sums: Let $\mu$ be an ordinary multiindex, then

$$
\begin{aligned}
& \left(\sum_{\underline{\mu}, \underline{\nu}} \frac{\left(4 \sqrt{6 M_{E}}\right)^{|\underline{\mu}|+|\underline{\nu}|}}{\sqrt{\underline{\mu} \underline{\underline{\nu}} \underline{\underline{\nu}}}} t^{\circ} \underline{\mu}+\underline{\nu}\right)=\left(\sum_{\mu} \frac{\left(4 \sqrt{6 M_{E}}\right)^{|\mu|}}{\sqrt{\mu !}} \dot{t}^{\mu}\right)^{4 M} \\
& \leq\left(\sum_{k=0}^{\infty} \frac{\left(4 \sqrt{6 M_{E}}\right)^{k}}{\sqrt{k !}} \sum_{\mu,|\mu|=k} \frac{|\mu| !}{\mu !} t^{\mu}\right)^{4 M} \\
& \leq\left(\sum_{k=0}^{\infty} \frac{\left(4 \sqrt{6 M_{E}}\|\stackrel{\circ}{T}\|_{1}\right)^{k}}{\sqrt{k !}}\right)^{4 M} \text {, }
\end{aligned}
$$

where in the second step we made use of the fact that the multinomial coefficients are greater than or equal to one and in the last step we used the multinomial formula (B.1.4). Clearly, the last sum is convergent. (As a matter of fact it would suffice to consider $k \leq M_{E}$, since $S_{\underline{\mu}, \underline{\underline{\nu}}, \hat{\alpha}, \hat{\beta}}$, given by formula ([F.2.11), vanishes for $|\underline{\mu}|>M_{E}$ or $\left.|\underline{\nu}|>M_{E}\right)$. As for the sum w.r.t. $\hat{\alpha}, \hat{\beta}$ on the r.h.s. of (F.3.10), it suffices to study the case $\left|\hat{\alpha}^{+}\right| \neq 0$. Then it factorizes into four independent sums and we discuss here one of the factors

$$
\begin{aligned}
\sum_{\hat{\alpha}^{+},\left|\hat{\alpha}^{+}\right| \neq 0} \sqrt{\frac{\left|\hat{\alpha}^{+}\right| !}{\hat{\alpha}^{+} !}} & (4 \sqrt{3 M} g(\delta))^{\left|\hat{\alpha}^{+}\right|} \hat{t}^{\hat{\alpha}^{+}} \\
& =\sum_{\hat{\alpha}^{+},\left|\hat{\alpha}^{+}\right| \neq 0}(4 \sqrt{3 M} g(\delta))^{\left|\hat{\alpha}^{+}\right|} \sqrt{\frac{\left(\left|\alpha_{1,2}^{+}\right|+\cdots+\left|\alpha_{M-1, M}^{+}\right|\right) !}{\alpha_{1,2}^{+} ! \ldots \alpha_{M-1, M}^{+} !}} t^{\hat{\alpha}^{+}} \\
\leq & \sum_{\hat{\alpha}^{+},\left|\hat{\alpha}^{+}\right| \neq 0}\left(4 \sqrt{3 M^{3}} g(\delta)\right)^{\left|\hat{\alpha}^{+}\right|} \frac{\left|\alpha_{1,2}^{+}\right| !}{\alpha_{1,2}^{+} !} \ldots \frac{\left|\alpha_{M-1, M}^{+}\right| !}{\alpha_{M-1, M}^{+} !} t^{\hat{\alpha}^{+}} \\
\leq & \sum_{\sum_{1,2}^{+}, \ldots, k_{M-1, M}^{+}} 1 \leq i<j \leq M \\
\leq & \sum_{\substack{k_{1,2}^{+}, \ldots, k_{M-1, M}^{+} \\
\sum_{1 \leq i<j \leq M} k_{i, j}^{+} \neq 0}}\left(4 \sqrt{3 M^{3}} g(\delta)\right)^{k_{i, j}^{+}} \sum_{\alpha_{i, j}^{+},\left|\alpha_{i, j}^{+}\right|=k_{i, j}^{+}} \frac{\left|\alpha_{i, j}^{+}\right| !}{\alpha_{i, j}^{+} !} t^{\alpha_{i, j}^{+}}
\end{aligned}
$$


In the second step we made use of the fact that

$$
\frac{\left(\left|\alpha_{1,2}^{+}\right|+\cdots+\left|\alpha_{M-1, M}^{+}\right|\right) !}{\left|\alpha_{1,2}^{+}\right| ! \ldots\left|\alpha_{M-1, M}^{+}\right| !} \leq M^{2\left(\left|\alpha_{1,2}^{+}\right|+\cdots+\left|\alpha_{M-1, M}^{+}\right|\right)}
$$

and in the last step we exploited the multinomial formula (B.1.4). The last expression on the r.h.s. of (F.3.12) is a convergent geometric series for sufficiently large $\delta$ and it tends to zero with $\delta \rightarrow \infty$, since $\lim _{\delta \rightarrow \infty} g(\delta)=0$. 


\section{Bibliography}

[Ar] H. Araki, Mathematical Theory of Quantum Fields. Oxford Science Publications, 1999.

[AH67] H. Araki and R. Haag, Collision cross sections in terms of local observables. Commun. Math. Phys. 4 (1967), 77-91.

[AHR62] H. Araki, K. Hepp and D. Ruelle, On the asymptotic behavior of Wightman functions in spacelike directions. Helv. Phys. Acta 35 (1962), 164-174.

[AB88] W. Arendt and C. J. K. Batty, Tauberian theorems and stability of oneparameter semigroups. Trans. Amer. Math. Soc. 309 (1988), 837-852.

[AB97] W. Arendt and C. J. K. Batty, Almost periodic solutions of first and second order Cauchy problems. J. Differential Equations 137 (1997), 363-383.

[AB99] W. Arendt and C. J. K. Batty, Asymptotically almost periodic solutions of inhomogeneous Cauchy problems on the half-line. Bull. London Math. Soc. 31 (1999), no. 3, 291-304.

[Ar75] W. Arveson, On groups of automorphisms of operator algebras. J. Funct. Anal. 15 (1974), 217-243.

[Ar82] W. Arveson, The harmonic analysis of automorphism groups. In Operator algebras and applications, Part I (Kingston, Ont., 1980), Proc. Sympos. Pure Math., 38, Amer. Math. Soc., Providence, R.I.,1982.D., pp. 199-269.

[BPh90] C. Batty and Vũ Quôc Phóng, Stability of individual elements under oneparameter semigroups. Trans. Amer. Math. Soc. 322 (1990), 805-818.

[Ba78] A. G. Baskakov, Spectral tests for the almost periodicity of the solutions of functional equations. Math. Notes 24 (1978), no. 1-2, 606-612.

[Bor65] H.-J. Borchers, On the vacuum state in quantum field theory II. Commun. Math. Phys. 1 (1965), 57-79.

[Bor70] H.-J. Borchers, Strongly continuous automorphism groups on $C^{*}$-algebras. Cargèse lecture notes in theoretical physics, 1969. D. Kastler ed. New York, London, Paris: Gordon and Breach, 1970.

[BBS01] H.-J. Borchers, D. Buchholz and B. Schroer, Polarization-free generators and the S-matrix. Commun. Math. Phys. 219 (2001), 125-140. 
[BHS63] H.-J. Borchers, R. Haag and B. Schroer, The vacuum state in quantum field theory. Nuovo Cimento 29 (1963), 148-162.

[Bos00] H. Bostelmann, Lokale Algebren und Operatorprodukte am Punkt. $\mathrm{PhD}$ Thesis, Universität Göttingen, 2000;

http://webdoc.sub.gwdg.de/diss/2000/bostelmann/

[Bos05.1] H. Bostelmann, Phase space properties and the short distance structure in quantum field theory. J. Math. Phys. 46 (2005), 052301-052318.

[Bos05.2] H. Bostelmann, Operator product expansions as a consequence of phase space properties. J. Math. Phys. 46 (2005), 082304-082317.

[BR] O. Bratelli and D. W. Robinson, Operator algebras and quantum statistical mechanics I. Springer-Verlag, 1979.

[Bu75] D. Buchholz, Collision theory for massless fermions. Commun. Math. Phys. 42 (1975), 269-279.

[Bu77] D. Buchholz, Collision theory for massless bosons. Commun. Math. Phys. 52 (1977), 147-173.

[Bu86] D. Buchholz, Gauss' law and the infraparticle problem. Phys. Lett. B 174 (1986), 331-334.

[Bu87] D. Buchholz, Particles, infraparticles and the problem of asymptotic completeness. In: VIIIth International Congress on Mathematical Physics. Marseille 1986. Singapore: World Scientific 1987.

[Bu90] D. Buchholz, Harmonic analysis of local operators. Commun. Math. Phys. 129 (1990), 631-641.

[Bu94] D. Buchholz, On the manifestations of particles. pp.177-202 in: Mathematical Physics Towards the 21st Century. Proceedings Beer-Sheva 1993, R.N. Sen and A. Gersten eds., Ben-Gurion University of the Negev Press 1994.

[Bu96] D. Buchholz, Phase space properties of local observables and structure of scaling limits. Ann. Inst. H. Poincaré 64 (1996), 433-459.

[BD95] D. Buchholz and C. D'Antoni, Phase space properties of charged fields in theories of local observables. Rev. Math. Phys. 7 (1995), 527-557.

[BDL87] D. Buchholz, C. D'Antoni, and R. Longo, The universal structure of local algebras. Commun. Math. Phys. 111 (1987), 123-135.

[BDL90] D. Buchholz, C. D'Antoni and R. Longo, Nuclear maps and modular structures II: applications to quantum field theory. Commun. Math. Phys. 129 (1990), 115-138.

[BF82] D. Buchholz and K. Fredenhagen, Locality and the structure of particle states. Commun. Math. Phys. 84 (1982), 1-54. 
[BJ89] D. Buchholz and P. Junglas, On the existence of equilibrium states in local quantum field theory. Commun. Math. Phys. 121 (1989), 255-270.

[BJa87] D. Buchholz and P. Jacobi, On the nuclearity condition for massless fields. Lett. Math. Phys. 13 (1987), 313-323.

[BP90] D. Buchholz and M. Porrmann, How small is the phase space in quantum field theory? Ann. Inst. H. Poincaré 52 (1990), 237-257.

[BPS91] D. Buchholz, M. Porrmann and U. Stein, Dirac versus Wigner: Towards a universal particle concept in quantum field theory. Phys. Lett. B 267 (1991), 377-381.

[BWi86] D. Buchholz and E. H. Wichmann, Causal independence and the energy-level density of states in local quantum field theory. Commun. Math. Phys. 106 (1986), 321-344.

[BWa92] D. Buchholz and R. Wanzenberg, The realm of the vacuum. Commun. Math. Phys. 143 (1992), 577-589.

[BY87] D. Buchholz and J. Yngvason, Generalized nuclearity conditions and the split property in quantum field theory. Lett. Math. Phys. 23 (1987), 159-167.

[Br03] J. Bros, A proof of Haag-Swieca's compactness property for elastic scattering states. Commun. Math. Phys. 237 (2003), 289-308.

[Co73] A. Connes, Une classification des facteurs de type III. Ann. Sci. Ecole Norm. Sup, 6 (1973), 133-252.

[CD82] M. Combescure and F. Dunlop, Three-body asymptotic completeness for $P(\phi)_{2}$ models. Commun. Math. Phys. 85 (1982), 381-418.

[CFP07] T. Chen, J. Froehlich and A. Pizzo, Infraparticle scattering states in non-relativistic QED: I. The Bloch-Nordsieck paradigm. Preprint: arXiv:0709.2493.

[DLZ81] C. D'Antoni, R. Longo and L. Zsido, A spectral mapping theorem for locally compact groups of operators. Pacific J. Math. 103 (1982), 17-24.

[DG] J. Dereziński and C. Gerard, Scattering theory of classical and quantum Nparticle systems. Springer 1997.

[De93] J. Dereziński, Asymptotic completeness of long-range $N$-body quantum systems. Ann. of Math. 138 (1993), 427-476.

[Dy05] W. Dybalski, Haag-Ruelle scattering theory in presence of massless particles. Lett. Math. Phys. 72 (2005), 27-38.

[Dy08.1] W. Dybalski, A sharpened nuclearity condition and the uniqueness of the vacuum in QFT. Commun. Math. Phys. 283 (2008), 523-542.

[Dy08.2] W. Dybalski, A sharpened nuclearity condition for massless fields. Lett. Math. Phys. 84 (2008), 217-230. 
[En75] V. Enss, Characterization of particles by means of local observables. Commun. Math. Phys. 45 (1975), 35-52.

[En78] V. Enss, Asymptotic completeness for quantum mechanical potential scattering. Commun. Math. Phys. 61 (1978), 285-291.

[Ex99] R. Exel, Unconditional integrability for dual actions. Bol. Soc. Brasil. Mat. (N.S.) 30 (1999), 99-124.

[Ex00] R. Exel, Morita-Rieffel equivalence and spectral theory for integrable automorphism groups of $C^{*}$-algebras. J. Funct. Anal. 172 (2000), 404-465.

[Ev76] D. Evans, On the spectrum of a one parameter strongly continuous representation. Math. Scand. 39 (1976), 80-82.

[FH81] K. Fredenhagen and J. Hertel, Local algebras of observables and pointlike localized fields. Commun. Math. Phys. 80 (1981), 555-561.

[GJ] J. Glimm and A. Jaffe, Quantum physics - a functional integral point of view. $2^{\text {nd }}$ ed., Springer, 1981.

[GJ70] J. Glimm and A. Jaffe, Quantum Field Theory Models. In: C. DeWitt and R. Stora eds. Statistical Mechanics and Quantum Field Theory. Université de Grenoble - Summer School of Theoretical Physics. Les Houches 1970.

[GJS73] J. Glimm, A. Jaffe and T. Spencer, The particle structure of the weakly coupled $P(\phi)_{2}$ model and other applications of high temperature expansions: Part I. Physics of quantum field models. Part II. The cluster expansion. In: Constructive quantum field theory. (Erice, 1973), G. Velo, A. S. Wightman eds. Berlin, Heidelberg, New York: Springer 1973.

[Gr90] G. M. Graf, Asymptotic completeness for N-body short-range quantum systems: a new proof. Commun. Math. Phys. 132 (1990), 73-101.

[Ha58] R. Haag, Quantum field theories with composite particles and asymptotic conditions. Phys. Rev. 112 (1958), 669-673.

[Ha] R. Haag, Local Quantum Physics. Springer 1992.

[Hal] P. R. Halmos, Measure Theory. D. Van Nostrand Company 1950.

[HS65] R. Haag and J.A. Swieca, When does a quantum field theory describe particles? Commun. Math. Phys. 1 (1965), 308-320.

[He65] K. Hepp, On the connection between the LSZ and Wightman quantum field theory. Commun. Math. Phys. 1 (1965), 95-111.

[He66] K. Hepp, On the connection between Wightman and LSZ quantum field theory. In: M. Chretien and S. Deser eds. Proc. Brandeis University Summer Institute in Theoretical Physics, vol.1, New York: Gordon and Breach 1966, pp. 137246. 
[Her71] I. Herbst, One-particle operators and local internal symmetries. J. Math. Phys. 12 (1971), 2480-2490.

[Herd07] A. Herdegen, Infrared problem and spatially local observables in electrodynamics. Ann. Henri Poincaré 9 (2008), 373-401.

[Hu99] Sen-Zhong Huang, Completeness of eigenvectors of group representations of operators whose Arveson spectrum is scattered. Proc. Amer. Math. Soc. 127 (1999), 1473-1482.

[Ja] H. Jarchow, Locally Convex Spaces. Stuttgart: B. G. Teubner, 1981.

[Jo92] P. E. T. Jorgensen, Spectral theory of one-parameter groups of isometries. J. Math. Anal. Appl. 168 (1992), 131-146.

[Jo82] P. E. T. Jorgensen, Spectral theory for infinitesimal generators of oneparameter groups of isometries: The mini-max principle and compact perturbations. J. Math. Anal. Appl. 90 (1982), 347-370.

[Ko84] H. Kosaki, On the continuity of the map $\phi \rightarrow|\phi|$ from the predual of a $W^{*}$ algebra. J. Funct. Anal. 59 (1984), 123-131.

[LN] K. B. Laursen and M.M. Neumann, An introduction to local spectral theory. Clarendon Press 2000.

[Le08] G. Lechner, Construction of quantum field theories with factorizing Smatrices. Commun. Math. Phys. 277 (2008), 821-860.

[LSZ55] H. Lehmann, K. Symanzik and W. Zimmermann Zur Formulierung quantisierter Feldtheorien. Nuovo Cim. 1 (1955), 205-225.

[Lo77] R. Longo, Some aspects of $C^{*}$-dynamics. In: Algèbres d'opérateurs et leur applications en physique mathèmatique. Colloques Internationaux du C.N.R.S. $\mathrm{n}^{\circ} 274,1977$.

[MS85] G. Morchio, F. Strocchi, Infrared problem, Higgs phenomenon and long range interactions. In: Fundamental problems of gauge field theory. Erice lecture notes 1985 .

[Me01] R. Meyer, Generalized fixed point algebras and square-integrable group actions. J. Funct. Anal. 186 (2001), 167-195.

[Ne92] M. M. Neumann, Banach algebras, decomposable convolution operators, and a spectral mapping property. In: Function spaces (Edwardswille, IL, 1990), 307-323, Lecture Notes in Pure and Appl. Math., 136, Dekker, New York, 1992.

[Ne98] M. M. Neumann, Natural spectrum, natural local spectra, and spectral mapping theorems for multipliers on Banach algebras. In: Banach algebras '97 (Blaubeuren), 377-395, de Gruyter, Berlin, 1998.

[Pe] G. K. Pedersen, $C^{*}$-algebras and their automorphism groups. London, New York, San Francisco: Academic Press, 1979. 
[Ph93] Vũ Quôc Phóng, On the spectrum, complete trajectories and asymptotic stability of linear semi-dynamical systems. J. Differential Equations 105 (1993), $30-45$.

[Po04.1] M. Porrmann, Particle weights and their disintegration I. Commun. Math. Phys. 248 (2004), 269-304.

[Po04.2] M. Porrmann, Particle weights and their disintegration II. Commun. Math. Phys. 248 (2004), 305-333.

[Ri90] M. A. Rieffel, Proper actions of groups on $C^{*}$-algebras. In: Mappings of operator algebras (Philadelphia, PA,1988), pp.141-182, Birkhäuser Boston, Boston, MA, 1990.

[RS1] M. Reed and B. Simon, Methods of modern mathematical physics. Part I: Functional analysis. Academic Press, New York, London, 1972.

[RS2] M. Reed and B. Simon, Methods of modern mathematical physics. Part II: Fourier analysis, self-adjointness. Academic Press, New York, San Francisco, London, 1975.

[Ru62] D. Ruelle, On the asymptotic condition in quantum field theory. Helv. Phys. Acta 35 (1962), 147-163.

[RS90] W. M. Ruess and W. H. Summers, Weakly almost periodic semigroups of operators. Pacific. J. Math. 143 (1990), 175-193.

[RS92] W. M. Ruess and W. H. Summers, Weak almost periodicity for semigroups of operators. J. Math. Anal. Appl. 164 (1992), 242-262.

[Sa] S. Sakai, $C^{*}$-algebras and $W^{*}$-algebras. Springer-Verlag, Berlin, Heidelberg, New York, 1971.

[Sch63] B. Schroer, Infrateilchen in der Quantenfeldtheorie. Fortschr. Phys. 11 (1963), $1-32$.

[SiSo87] I. M. Sigal and A. Soffer, The N-particle scattering problem: asymptotic completeness for short-range systems. Ann. of Math. 126 (1987), 35-108.

[Sp] H. Spohn, Dynamics of charged particles and their radiation field. Cambridge University Press, 2004.

[St89] U. Stein, Zur Konstruktion von Streuzuständen mit Hilfe lokaler Observabler. PhD Thesis, Universität Hamburg 1989.

[SZ76] T. Spencer and F. Zirilli, Scattering states and bound states in $\lambda P(\phi)_{2}$. Commun. Math. Phys. 49 (1976), 1-16.

[SW80] R. F. Streater and A. S. Wightman, PCT, spin and statistics, and all that. $3^{\text {rd }}$ ed., Princeton University Press, 1980.

[Wi39] E. P. Wigner, On unitary representations of the inhomogeneous Lorentz group. Ann. Math. 40 (1939), 149-204. 


\section{Notational Conventions}

Minkowski space notation: We work in Minkowski spacetime $\mathbb{R}^{s+1}$. We use the Minkowski metric $\eta_{\mu \nu}$ with signature $(+, \underbrace{-, \ldots,-}_{s})$. The Minkowski scalar product of two four-vectors is given by $p \cdot x=p^{0} x^{0}-\vec{p} \vec{x}$, where $\vec{p} \vec{x}=\sum_{i=1}^{s} p_{i} x_{i}$.

Fourier transform: Consider the Schwartz-class functions $f \in S\left(\mathbb{R}^{s+1}\right), g \in S\left(\mathbb{R}^{s}\right)$ and $h \in S(\mathbb{R})$. ( $h$ is understood as a function of time). We define their Fourier transforms as follows

$$
\begin{aligned}
\tilde{f}(p) & =(2 \pi)^{-\frac{s+1}{2}} \int d^{s+1} x e^{i p^{0} x^{0}-i \vec{p} \vec{x}} f\left(x^{0}, \vec{x}\right), \\
\tilde{g}(\vec{p}) & =(2 \pi)^{-\frac{s}{2}} \int d^{s} x e^{-i \vec{p} \vec{x}} g(\vec{x}), \\
\tilde{h}\left(p^{0}\right) & =(2 \pi)^{-\frac{1}{2}} \int d x^{0} e^{i p^{0} x^{0}} h\left(x^{0}\right) .
\end{aligned}
$$

The Fourier transforms of distributions $F \in S^{\prime}\left(\mathbb{R}^{s+1}\right), G \in S^{\prime}\left(\mathbb{R}^{s}\right)$ and $H \in S^{\prime}(\mathbb{R})$ are defined according to the relations $\tilde{F}(\tilde{f})=F(f), \tilde{G}(\tilde{g})=G(g)$ and $\tilde{H}(\tilde{h})=H(h)$. In the formal notation

$$
\begin{aligned}
\tilde{F}(p) & =(2 \pi)^{-\frac{s+1}{2}} \int d^{s+1} x e^{-i p^{0} x^{0}+i \vec{p} \vec{x}} F\left(x^{0}, \vec{x}\right), \\
\tilde{G}(\vec{p}) & =(2 \pi)^{-\frac{s}{2}} \int d^{s} x e^{i \vec{p} \vec{x}} G(\vec{x}), \\
\tilde{H}\left(p^{0}\right) & =(2 \pi)^{-\frac{1}{2}} \int d x^{0} e^{-i p^{0} x^{0}} H\left(x^{0}\right) .
\end{aligned}
$$


Additional conditions:

\begin{tabular}{|c|c|}
\hline Condition & Reference \\
\hline Condition $A$ & Page 63 \\
\hline Condition $A^{\prime}$ & Page 64 \\
\hline Condition $C_{\sharp}$ & Page 34 \\
\hline Condition $C_{b}$ & Page 43 \\
\hline Condition $C_{\natural}$ & Page 50 \\
\hline Condition $L^{(1)}$ & Page 27 \\
\hline Condition $L_{\sharp}^{(1)}$ & Page 30 \\
\hline Condition $L^{(2)}$ & Page 24 \\
\hline Condition $M$ & Page 64 \\
\hline Condition $N_{\sharp}$ & Page 53 \\
\hline Condition $N_{\natural}$ & Page 54 \\
\hline Condition $R$ & Page 37 \\
\hline Condition $S$ & Page 65 \\
\hline Condition $T$ & Page 29 \\
\hline Condition $V$ & Page 22 \\
\hline
\end{tabular}

\section{Frequently used symbols:}

\begin{tabular}{|c|c|c|}
\hline Symbol & Description & Reference \\
\hline$\|\cdot\|_{E, 2}$ & Square-integrability seminorm on $\hat{\mathfrak{A}}_{\mathrm{ac}}$ & Page 23 \\
\hline$\|\cdot\|_{E, 1}$ & Integrability seminorm on $\mathfrak{A}^{(1)}$ & Page 26 \\
\hline$\|\cdot\|_{p}$ & $p$-norm w.r.t. the norm $\|\cdot\|$ & Page 53 \\
\hline$\|\cdot\|_{p}$ & Norm on $L^{p}\left(\mathbb{R}^{s}\right) / p$-norm of an operator on $L^{2}\left(\mathbb{R}^{s}, d^{s} p\right)$ & Page 81,86 \\
\hline$\|\cdot\|_{x_{1}, \ldots, x_{N}}$ & Special norms on $\mathcal{L}\left(\mathcal{T}_{E}, X\right)$ & Page 54 \\
\hline$\|\cdot\|_{p, x_{1}, \ldots, x_{N}}$ & $p$-norm w.r.t. the norm $\|\cdot\|_{x_{1}, \ldots, x_{N}}$ & Page 54 \\
\hline$\|\cdot\|_{2, l}$ & Sobolev norms & Page 81 \\
\hline & Closure in $L^{2}\left(\mathbb{R}^{s}, d^{s} p\right)$ & Page 86 \\
\hline$a^{*}(\vec{p}), a(\vec{p})$ & Creation and annihilation operators of a mode $\vec{p}$ & Page 79 \\
\hline$a^{*}(f), a(f)$ & Creation and annihilation operators of a function $f$ & Page 77 \\
\hline$\underline{A}$ & Observable $A$ restricted to $\mathcal{H}^{(\mathrm{e})}$ & Page 78 \\
\hline$\overline{\hat{\mathfrak{A}}}$ & Algebra of local observables & Page $\overline{13}$ \\
\hline $\mathfrak{A}^{(\mathrm{d})}$ & Algebra of observables generated by derivatives of the free field & Page 78 \\
\hline$\underline{\mathfrak{A}}^{(\mathrm{e})}$ & $\begin{array}{l}\text { Algebra of observables of the even part of free field theory acting } \\
\text { on } \mathcal{H}^{(\mathrm{e})}\end{array}$ & Page 78 \\
\hline $\mathfrak{A}^{(\mathrm{e})}$ & $\begin{array}{l}\text { Algebra of observables of the even part of free field theory acting } \\
\text { on the (full) Fock space }\end{array}$ & Page 78 \\
\hline $\mathfrak{A}$ & Global algebra of observables & Page 13 \\
\hline$\stackrel{\mathfrak{A}}{(\mathcal{O})}$ & *-algebra of finite, linear combinations of Weyl operators & Page 88 \\
\hline $\mathfrak{A}(\mathcal{O})$ & Local algebra attached to $\mathcal{O}$ & Page 13 \\
\hline$\alpha_{x}$ & Translation automorphisms & Page 13 \\
\hline$\underline{\alpha}_{r}$ & Translation automorphisms of free field theory restricted to $\mathcal{H}^{(\mathrm{e})}$ & Page 78 \\
\hline
\end{tabular}




\begin{tabular}{|c|c|c|}
\hline$\widetilde{A}(\vec{p})$ & Fourier transform of a local observable & Page 15 \\
\hline$\hat{\mathfrak{A}}_{\mathrm{pp}}$ & Pure-point subspace of $\hat{\mathfrak{A}}$ & Page 20 \\
\hline$\hat{\mathfrak{A}}_{\mathrm{c}}$ & Continuous subspace of $\hat{\mathfrak{A}}$ & Page 22 \\
\hline $\mathfrak{A}_{\mathrm{c}}(\mathcal{O})$ & Continuous subspace of $\mathfrak{A}(\mathcal{O})$ & Page 22 \\
\hline$\hat{\mathfrak{A}}_{\mathrm{ac}}$ & Absolutely continuous subspace of $\hat{\mathfrak{A}}$ & Page 24 \\
\hline$\hat{\mathfrak{A}}_{\mathrm{pc}}$ & Point-continuous subspace of $\hat{\mathfrak{A}}$ & Page 24 \\
\hline $\mathfrak{A}^{(1)}$ & Space of integrable observables & Page 27 \\
\hline$\left(\mathfrak{A}(\mathcal{O})^{\times N}\right)^{*}$ & $N$-linear forms on $\mathfrak{A}(\mathcal{O})$ & Page 38 \\
\hline$B(\mathcal{H})$ & Bounded operators on $\mathcal{H}$ & Page 13 \\
\hline$\tilde{b}_{\kappa, r}^{ \pm}$ & Functions appearing in the Taylor expansion of $\tilde{F}^{ \pm}$ & Page 84 \\
\hline $\mathfrak{C}^{\prime}$ & Algebra of particle detectors & Page 30] \\
\hline $\mathbb{C}_{\text {sup }}^{N}$ & $\mathbb{C}^{N}$ equipped with the supremum norm & Page 48 \\
\hline$C_{0}^{\infty}\left(\mathbb{R}^{s}\right)$ & Smooth, compactly supported functions on $\mathbb{R}^{s}$ & \\
\hline$D_{B}$ & Vectors of bounded energy & Page 14 \\
\hline$D_{F}$ & Subspace of finite particle vectors in Fock space & Page 77 \\
\hline$D_{S}$ & Subspace of vectors with Schwartz-class wavefunctions in $D_{F}$ & Page 79 \\
\hline$D\left(\mathcal{O}_{r}\right)$ & Smooth functions supported in $\mathcal{O}_{r}$ & Page 77 \\
\hline$\left\{e_{j}\right\}_{1}^{\infty}$ & Basis of $J$-invariant eigenvectors of $T$ & Page 88 \\
\hline$\Phi_{\mathrm{FH}}$ & Field content & Page 28 \\
\hline$\Phi_{\mathrm{FH}}^{(\mathrm{d})}$ & Field content of $\left(\mathfrak{A}^{(\mathrm{d})}, \alpha, \mathcal{H}\right)$ & Page 80 \\
\hline$\Phi_{\mathrm{FH}}^{(\mathrm{e})}$ & Field content of $\left(\underline{\mathfrak{A}}^{(\mathrm{e})}, \underline{\alpha}, \mathcal{H}^{(\mathrm{e})}\right)$ & Page 80 \\
\hline $\mathcal{F}(V, W)$ & Finite rank maps from Banach space $V$ to $W$ & Page 34 \\
\hline $\mathcal{F}(V \times \Gamma, W)$ & Finite rank maps form $V \times \Gamma$ to $W$ & Page 38 \\
\hline$\varphi_{\underline{\vec{x}}}$ & Special elements of $\left(\mathfrak{A}(\mathcal{O})^{\times N}\right)^{*}$ & Page 39 \\
\hline$\phi_{ \pm}$ & Canonical field and momentum & Page 77 \\
\hline$: \phi_{ \pm}^{n}:$ & Wick powers of the fields $\phi_{ \pm}$ & Page 80 \\
\hline$\Gamma_{N, \delta}^{-}$ & Set of admissible configurations of bounded regions & Page 38 \\
\hline$H$ & Hamiltonian & Page 13 \\
\hline $\mathcal{H}$ & Hilbert space & Page 13 \\
\hline $\mathcal{H}^{(\mathrm{e})}$ & Even part of the Fock space & Page 78 \\
\hline$\chi_{E}$ & Approximate characteristic function of $\left\{\vec{p} \in \mathbb{R}^{s} \mid \omega(\vec{p}) \leq E\right\}$ & Page 84 \\
\hline$\chi\left(\mathcal{O}_{r}\right)$ & Approximate characteristic function of $\mathcal{O}_{r}$ & Page 84,109 \\
\hline$\tilde{h}_{\kappa, E}^{ \pm}$ & Functions appearing in the Taylor expansion of $\tilde{F}^{ \pm}$ & Page 84 \\
\hline$h_{r}$ & Special functions from $D\left(\mathcal{O}_{r}\right)$ & Page 86 \\
\hline$\iota_{(\mathrm{e})}$ & Isometric embedding $\mathcal{T}^{(\mathrm{e})} \hookrightarrow \mathcal{T}$ & Page 79 \\
\hline$J$ & Complex conjugation in $L^{2}\left(\mathbb{R}^{s}, d^{s} x\right)$ & Page 77 \\
\hline$\kappa$ & $s$-index & Page 76 \\
\hline $\mathcal{L}(V, W)$ & Linear maps from Banach space $V$ to $W$ & Page 34 \\
\hline $\mathcal{L}(V \times \Gamma, W)$ & Maps form $V \times \Gamma$ to $W$, linear in the first argument & Page 38 \\
\hline $\mathcal{L}(V \times \Gamma, W)$ & Maps form $V \times \Gamma$ to $W$, linear in the first argument & Page 38 \\
\hline$\stackrel{\circ}{\mathcal{L}}_{r}, \stackrel{\circ}{L}_{r}^{ \pm}$ & Special subspaces of $L^{2}\left(\mathbb{R}^{s}, d^{s} p\right)$ & Page 77 \\
\hline$L^{2}\left(\mathbb{R}^{s}, d^{s} x\right)$ & Square-integrable functions on $\mathbb{R}^{s}$ & Page 81 \\
\hline$L^{2}\left(\mathbb{R}^{s}, d^{s} x\right)_{l}$ & Sobolev space & Page 81 \\
\hline $\mathcal{L}_{r}^{ \pm}$ & Special subspaces/projections on $L^{2}\left(\mathbb{R}^{s}, d^{s} p\right)$ & Page 86 \\
\hline $\bar{\mu}$ & Pair of multiindices & Page 76 \\
\hline $\mathcal{M}$ & Finite subset of pairs of multiindices & Page 89 \\
\hline
\end{tabular}




\begin{tabular}{|c|c|c|}
\hline $\mathcal{N}(\varepsilon)$ & $\varepsilon$-content of a map/set & Page 43, 49 \\
\hline $\mathcal{O}$ & Open bounded region of $\mathbb{R}^{s+1}$ & Page 13 \\
\hline $\mathcal{O}(r)$ & Double cone of radius $r$ & Page 14 \\
\hline $\mathcal{O}_{r}$ & Ball in $\mathbb{R}^{s}$ of radius $r$ & Page 14 \\
\hline$\omega_{0}$ & Vacuum state (or translationally invariant state) & Page 15 \\
\hline $\operatorname{ord}(A)$ & Infrared order of $A \in \hat{\mathfrak{A}}_{\mathrm{c}}$ & Page 24 \\
\hline $\operatorname{Ord}(A)$ & $\left\{\operatorname{ord}(A) \mid A \in \hat{\mathfrak{A}}_{\mathrm{c}}\right\}$ & Page $\overline{24}$ \\
\hline$\omega^{\vec{x}}$ & Special elements of $\mathfrak{A}(\mathcal{O})^{*} \otimes \mathbb{C}_{\text {sup }}^{N}$ & Page 50 \\
\hline$\omega(\vec{p})$ & $\sqrt{|\vec{p}|^{2}+m^{2}}$ & Page 76 \\
\hline$P_{+}^{\uparrow}$ & Poincaré group & Page 13 \\
\hline$\vec{P}+$ & Momentum operators & Page 13 \\
\hline$P_{E}$ & Spectral projection of $H$ on $\left\{\omega \in \mathbb{R}_{+} \mid \omega \leq E\right\}$ & Page 14 \\
\hline $\mathfrak{P}$ & Particle content & Page 28 \\
\hline$\Pi_{E}, \Theta_{E}, \Xi_{\beta}$ & Maps appearing in Condition $C_{\sharp}$ & Page 34 \\
\hline$\Pi_{E, N, \delta}$ & Maps appearing in Lemma 3.2 .1 & Page 38 \\
\hline$\Pi_{E, N, \delta}^{\mathrm{c}}$ & Map appearing in Condition $C_{\mathrm{b}}$ & Page 42 \\
\hline$P_{(p, r)}^{L, i v 0}$ & Spectral projection on the ball of radius $r$ centered at $r$ & Page 47 \\
\hline$\Pi_{E}^{\mathrm{c}}$ & Maps appearing in Condition $N_{\sharp}$ & Page 54 \\
\hline$\pi_{(\mathrm{e})}^{L}$ & Representation of $\mathfrak{A}^{(\mathrm{e})}$ in $B\left(\mathcal{H}^{(\mathrm{e})}\right)$ & Page 78 \\
\hline$Q_{E}$ & Projection on vectors of energy below $E$ in $L^{2}\left(\mathbb{R}^{s}, d^{s} p\right)$ & Page 86 \\
\hline$R$ & $(1+H)^{-1} /$ Parameter appearing in Lemma A.3.2 & Page 28,71 \\
\hline$R^{(2)}$ & Map appearing in Theorem C.1.1 & Page $\overline{95}$ \\
\hline$S\left(\mathbb{R}^{s}\right)$ & Schwartz-class functions on $\mathbb{R}^{s}$ & \\
\hline$S_{E}$ & States from $\mathcal{T}_{E}$ & Page 14 \\
\hline$S_{E}(\mathcal{O})$ & Special sets from the ranges of $\Pi_{E}$ & Page 49 \\
\hline$S_{E, N . \delta}(\mathcal{O})$ & Special sets from the ranges of $\Sigma_{E, N, \delta}$ & Page 49 \\
\hline $\operatorname{Sp}_{B}\left(\alpha_{\mathbb{R}^{s+1}}\right)$ & Arveson spectrum of $B \in \mathfrak{A}$ w.r.t. $\alpha_{x}$ & Page 14 \\
\hline$\sigma_{\varphi}^{(t)}$ & Asymptotic functional approximants & Page 28 \\
\hline$\sigma_{\varphi}^{(+)}$ & Asymptotic functionals & Page 28 \\
\hline$\sigma_{\mu^{+}, \mu^{-}}$ & Special functionals on $\mathfrak{A}(\mathcal{O})$ in free field theory & Page 81 \\
\hline $\bar{\sigma}_{\alpha^{+}, \alpha^{-}}^{(r)}$ & Special functionals on $\mathfrak{A}(\mathcal{O})$ in free field theory & Page 90 \\
\hline$\Sigma_{E, N, \delta}$ & Maps appearing in Condition $C_{\natural}$ & Page 48 \\
\hline$s$ & Dimension of space & \\
\hline $\mathcal{S}$ & Suitable subset of $\mathbb{N}_{0}^{2}$ & Page 88 \\
\hline$S_{\bar{\mu}, \bar{\nu}}$ & Operators appearing in expansion (B.5.20) of the map $\Theta_{E, 1}$ & Page 90 \\
\hline$S_{\bar{\mu}, \bar{\nu}}$ & Operators appearing in expansion (B.5.37) of the map $\Theta_{E, 2}$ & Page 93 \\
\hline $\mathcal{T}^{\mu, \nu}$ & Trace-class operators on $\mathcal{H}$ & Page 14 \\
\hline $\mathcal{T}_{E}$ & $P_{E} \mathcal{T} P_{E}$ & Page 14 \\
\hline $\mathcal{T}_{E}^{+}$ & Positive elements from $\mathcal{T}_{E}$ & Page 14 \\
\hline $\mathcal{T}_{\infty}$ & Functionals of polynomially damped energy & Page 28 \\
\hline $\mathcal{T}_{(p, r)}$ & $P_{(p, r)} \mathcal{T} P_{(p, r)}$ & Page 47 \\
\hline $\mathcal{T}^{(\mathrm{e})}$ & Predual of $B\left(\mathcal{H}^{(\mathrm{e})}\right)$ & Page 79 \\
\hline$\tau_{\bar{\mu}, \bar{\nu}}$ & Functionals appearing in expansion (B.5.37) of the map $\Theta_{E, 2}$ & Page 93 \\
\hline$\stackrel{\vee}{\tau}_{\bar{\mu}, \bar{\nu}}(r)$ & Functionals appearing in expansion (B.5.20) of the map $\stackrel{\vee}{\Theta}_{E, 1}$ & Page 90 \\
\hline$\hat{\tau}_{\alpha^{+}, \alpha^{-}}^{(r)}$ & Functionals appearing in expansion (B.5.15) of the map $\hat{\Theta}_{E, 1}$ & Page 90 \\
\hline
\end{tabular}


$T^{00}$

$T_{E, \pm}, T_{h, \pm}$

$T$

$\left\{t_{j}\right\}_{1}^{\infty}$

$\Theta_{E, 1}$

$\Theta_{E, 2}$

$\hat{\Theta}_{E, 1}$

$\stackrel{\vee}{\Theta}_{E, 1}$

$U$

$W(f)$
Stress-energy tensor

Suitable nuclear operators on $L^{2}\left(\mathbb{R}^{s}, d^{s} p\right)$

$\left(\left|T_{E,+}\right|^{2}+\left|T_{E,-}\right|^{2}+\left|T_{h,+}\right|^{2}+\left|T_{h,-}\right|^{2}\right)^{\frac{1}{2}}$

Eigenvalues of $T$

Map appearing in decomposition (B.5.3) of $\Theta_{E}$

Map appearing in decomposition (B.5.3) of $\Theta_{E}$

Map appearing in decomposition (B.5.11) of $\Theta_{E}$

Map appearing in decomposition (B.5.11) of $\Theta_{E}$

Unitary representation of translations in $\mathcal{H}$

Weyl operator
Page 29]

Page 86]

Page 87

Page 88

Page 88

Page 88

Page 89

Page 89

Page 13

Page 78 


\section{Acknowledgements}

I would like to thank Prof. D. Buchholz for formulating an interesting problem in the field of particle structures and automorphism groups, and giving me the opportunity to work on it. Without his advice and encouragement this work would not have been possible. In particular, I am indebted to him for suggesting to me the approach to the study of the vacuum structure pursued in Section 3.3 and for pointing out to me his observations summarized in Section 2.1 .

I am also grateful to Prof. K. Fredenhagen for his immediate consent to write the additional report.

Thanks are due to my MSc Thesis adviser, Prof. J. Derezinski, for introducing me to the subject of scattering in quantum field theory and to Prof. J. Bros for an outline of its early developments. I am also grateful to Prof. S. Gierowski for pointing out to me the value of abstract thinking.

In the course of this work I benefited from discussions with many past or present members and visitors of the Quantum Field Theory Group in Göttingen. In particular, I would like to thank Jan Schlemmer, Ansgar Schneider, Antonia Miteva, Helmut Hölzler, Gandalf Lechner, Nikolay Nikolov, Katarzyna Rejzner, Julian Pook and Daniela Cadamuro.

Many thanks to my family: My parents for their numerous visits to Göttingen and holidays we spent together. My grandparents for encouraging telephone conversations. My brother Łukasz for endless discussions about art and science.

Finally, I would like to thank great friends who supported me at various stages of this work: Jasmin Kernspecht, Kostas Daoulas, Aneta Kiebała, Anna Podlewska and Christian Podlewski.

Financial support from EC Research Training Network 'Quantum Spaces - Noncommutative Geometry', Deutsche Forschungsgemeinschaft and Graduiertenkolleg 'Mathematische Strukturen in der modernen Quantenphysik' is gratefully acknowledged. I also acknowledge travel grants from the Research Institute in Oberwolfach, Max Planck Institute for Mathematics in Bonn and Universities of Mainz, Hamburg and Leipzig, as well as from Wilhelm und Else Heraeus-Stiftung. A fellowship supported by the Austrian Federal Ministry of Science and Research, the High Energy Physics Institute of the Austrian Academy of Sciences and the Erwin Schrödinger International Institute of Mathematical Physics supported my stay at the 4th Vienna Central European Seminar on Particle Physics and Quantum Field Theory. 


\section{Lebenslauf}

Wojciech Jan Dybalski

Goßlerstraße 33B WE 29

37075 Göttingen

Tel. 0551 / 2097264

dybalski@theorie.physik.uni-goettingen.de

Geboren am 27. Januar 1980 in Warschau

Polnische Staatsangehörigkeit

Juni 1998 Abitur am I Karol Marcinkowski Gymnasium, Poznań

Oktober 1998 Studium der Physik an der Warschauer Universität

bis Juni 2003

Juni 2003 Diplomprüfung. Titel der Diplomarbeit:

,Streuung von massiven Teilchen in der Quantenfeldtheorie”.

Anleitung: Prof. Dr. J. Dereziński

September 2003 Wissenschaftlicher Mitarbeiter am Nationalen Forschungsrat (NRC),

bis Oktober 2004 Ottawa sowie an der Universität Ottawa, Canada

seit November 2004 Bearbeitung des Promotionsthemas:

„Automorphismengruppen und Teilchenstruktur".

Betreuer der Dissertation: Prof. Dr. D. Buchholz 